\title{
One-Step Synthesis of Diverse Pyridine-containing Heterocycles with 3-Ethoxycyclobutanones at Room Temperature
}

Yun Lin ${ }^{\mathrm{a}}$, Xinglin Yang ${ }^{\mathrm{a}}$, Weidong $\mathrm{Pan}^{\mathrm{b}, *}$ and $\mathrm{Yu} \mathrm{Rao}^{\mathrm{a}, *}$

${ }^{a}$ MOE Key Laboratory of Protein Sciences, Department of Pharmacology and Pharmaceutical Sciences, School of Medicine and School of Life Sciences, Tsinghua University, Beijing 100084, China; ${ }^{\mathrm{b}}$ The Key Laboratory of Chemistry for Natural Products of Guizhou Province and Chinese Academy of Sciences, Guiyang 550002, PR China.

E-mail: yrao@ mail.tsinghua.edu.cn; wdpan@163.com

\begin{tabular}{|l|l|l|}
\hline \multicolumn{2}{|l|}{ Contents } & Page \\
\hline I. & Materials and Methods & 2 \\
\hline II. & General procedure & 2 \\
\hline III. & References & 2 \\
\hline IV & Data of products & $3-18$ \\
\hline
\end{tabular}




\section{Materials and Methods}

All commercial materials (Alfa Aesar, Aladdin, J\&K Chemical LTD., Beijing Ouhe Technology Co.Ltd.) were used without further purification. All solvents were analytical grade. Anhydrous dichloromethane, acetonitrile, 1, 2-dichloroethane were obtained through refluxing over $\mathrm{CaH}_{2}$ and redistillation. The ${ }^{1} \mathrm{H} N M R$ and ${ }^{13} \mathrm{C}$ NMR spectra were recorded on a $400 \mathrm{MHz}$ spectrometer in $\mathrm{CDCl}_{3}$ using TMS or solvent peak as a standard. All ${ }_{13} \mathrm{C}$ NMR spectra were recorded with complete proton decoupling. Mass spectra were recorded on a Thermo Fisher Orbitrap Fusion Mass Spectrometer (ESI). All IR spectra were recorded on a Bruker company equipment (ATR). All reactions were carried out in oven-dried glassware with a rubber seal. Analytical TLC was performed on Yantai Chemical Industry Research Institute silica gel 60 F254 plates and flash column chromatography was performed on Qingdao Haiyang Chemical Co. Ltd silica gel 60 (200-300mesh). The rotavapor was BUCHI's Rotavapor R-3. Substituted 3-ethoxycyclobutanones was prepared according to the literature reports. ${ }^{1,2}$

All the reactions were carried out under a nitrogen atmosphere except the optimization study involving oxygen. Silica gel plates (GF254) were used for TLC monitoring and silica gel (230-400 mesh) was used for flash column chromatography. All final compounds were characterized by 1H NMR, 13C NMR and Waters UPLC-mass spectroscopy. Copies of NMR spectra are included for all final compounds. 1H NMR and 13C NMR spectra were recorded on Bruker models Avance DPX $400(400 \mathrm{MHz})$ spectrometers. 1H NMR spectra are reported in parts per million (ppm) downfield of TMS and were measured relative to the signals for $\mathrm{CHCl}_{3}$ (7.26 ppm), Acetone-d6 (d 2.05) and DMSO-d6 (d 2.50). All 13C NMR spectra were reported in ppm and were obtained with $1 \mathrm{H}$ decoupling. In reporting spectral data, the format $(\delta)$ chemical shift (multiplicity, J values in $\mathrm{Hz}$, integration) was used with the following abbreviations: $\mathrm{s}=$ singlet, brs = broad singlet, $\mathrm{d}=$ doublet, $\mathrm{dd}=$ doublet of doublets, $\mathrm{t}=$ triplet, $\mathrm{q}=$ quartet, $\mathrm{m}=$ multiplet, brm = broad multiplet. MS analyses were carried out on Waters UPLC-Mass instrument.

\section{General procedure for the synthesis of heterocyclics}

Substituted heterocyclic amines (1.0 equiv, $0.30 \mathrm{mmol}$ ) and substituted 3-ethoxycyclobutanones (1.0 equiv) were dissolved in anhydrous dichloromethane $(2 \mathrm{ml})$ in a round bottom flask. Following that, $\mathrm{BF}_{3} \cdot \mathrm{OEt}_{2}(1.0$ equiv) was added slowly into the reaction solution. Then the reaction mixture was stirred at room temperature under atmosphere of Argon for 6-12 h. The reaction was monitored by TLC and LC-MS. After completion of the reaction, Et $3 \mathrm{~N}$ (1 2 drops) was added to quench the reaction. The reaction mixture was diluted with dichloromethane (15 ml) and washed once with saturated aqueous $\mathrm{NaHCO}_{3}(10 \mathrm{ml})$ and once with water $(10 \mathrm{ml})$. Then organic layer was dried over $\mathrm{MgSO}_{4}$ and concentrated on rotavapor under reduced pressure. Finally the residue was purified by silical gel column chromatography to give corresponding heterocyclic products.

\section{References}

[1] Matsuo , J.; Sasaki, S.; Hoshikawa, T.; Ishibashi, H. Org.Lett. 2009. 11. 3822.

[2] Matsuo J.;Okuno,R; Takeuchi, K.; Kawano,M; Ishibashi,H. Tetrahedron Lett. 2010. 51.3736 


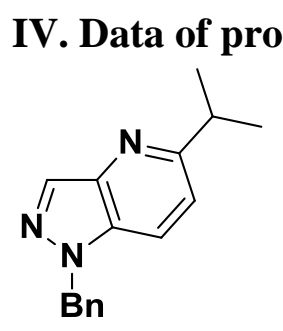

\section{1-benzyl-5-isopropyl-1H-pyrazolo[4,3-b]pyridine}

Following the general procedure II, amine $(104 \mathrm{mg}, 0.60 \mathrm{mmol}), \mathrm{BF}_{3} \cdot \mathrm{OEt}_{2}(73 \mathrm{ul}, 0.60 \mathrm{mmol})$ and 2,2-dimethyl 3-ethoxycyclobutanone (1.0 equiv) were used. The reaction mixture was stirred at room temperature for $9 \mathrm{~h}$ under Ar protection. After completion of the reaction, the residue was purified by silical gel column chromatography (Hexane: Ether =10:1). Finally, compound 3 (126 $\mathrm{mg}$, white solid) was isolated in $84 \%$ yield. ${ }^{1} \mathrm{H}-\mathrm{NMR}\left(400 \mathrm{MHz}, \mathrm{CDCl}_{3}\right) \delta(\mathrm{ppm}) 8.22(\mathrm{~s}, 1 \mathrm{H}), 7.55$ $(\mathrm{d}, \mathrm{J}=8.8 \mathrm{~Hz}, 1 \mathrm{H}), 7.28(\mathrm{~m}, 3 \mathrm{H}), 7.19(\mathrm{~d}, 2 \mathrm{H}), 7.14(\mathrm{~d}, \mathrm{~J}=8.8 \mathrm{~Hz}, 1 \mathrm{H}), 5.57(\mathrm{~s}, 2 \mathrm{H}), 3.19(\mathrm{~m}, 1 \mathrm{H}), 1.34$ $(\mathrm{d}, 6 \mathrm{H}) ;{ }^{13} \mathrm{C}-\mathrm{NMR}\left(100 \mathrm{MHz}, \mathrm{CDCl}_{3}\right) \delta$ (ppm) 163.4, 141.8, 136.5, 133.8, 131.6, 129.0, 128.1, 127.4, 119.2, 117.9, 53.9, 36.7, 23.0; HRMS (ESI) calcd for $\mathrm{C}_{16} \mathrm{H}_{17} \mathrm{~N}_{3}[\mathrm{M}+\mathrm{H}]^{+}:$252.1495, found 252.1502.

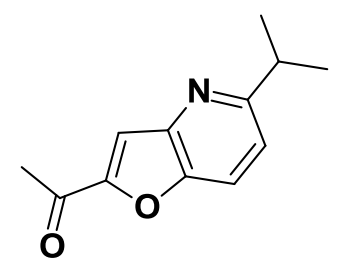

\section{4a. 1-(5-isopropylfuro[3,2-b]pyridin-2-yl)ethanone}

Following the general procedure $\mathbf{I I}$, amine $(0.30 \mathrm{mmol}), \mathrm{BF}_{3} \cdot \mathrm{OEt}_{2}(36 \mathrm{ul}, 0.30 \mathrm{mmol})$ and 2,2-dimethyl 3-ethoxycyclobutanone (1.0 equiv) were used. The reaction mixture was stirred at room temperature for $10 \mathrm{~h}$ under Ar protection. After completion of the reaction, the residue was purified by silical gel column chromatography (Hexane: Ether $=10: 1)$. Finally, compound 4a $(33$ $\mathrm{mg}$, colorless oil) was isolated in $55 \%$ yield. ${ }^{1} \mathrm{H}-\mathrm{NMR}\left(400 \mathrm{MHz}, \mathrm{CDCl}_{3}\right) \delta(\mathrm{ppm}) 7.98(\mathrm{~d}, \mathrm{~J}=8.0 \mathrm{~Hz}$, 1H), $7.44(\mathrm{~d}, 1 \mathrm{H}), 7.22(\mathrm{~d}, \mathrm{~J}=8.0 \mathrm{~Hz}, 1 \mathrm{H}), 3.19(\mathrm{~m}, 1 \mathrm{H}), 2.65(\mathrm{~s}, 3 \mathrm{H}), 1.37(\mathrm{~d}, 6 \mathrm{H}) ;{ }^{13} \mathrm{C}-\mathrm{NMR}(100$ $\left.\mathrm{MHz}, \mathrm{CDCl}_{3}\right) \delta(\mathrm{ppm}) 163.2,141.3,133.3,131.9,119.1,117.5,36.8,36.0,23.0 ;$ HRMS (ESI) calcd for $\mathrm{C}_{12} \mathrm{H}_{13} \mathrm{NO}_{2}[\mathrm{M}+\mathrm{H}]^{+}:$204.1019, found 204.1024.

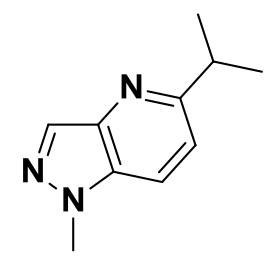

\section{4b. 5-isopropyl-1-methyl-1H-pyrazolo[4,3-b]pyridine}

Following the general procedure II, amine $(0.60 \mathrm{mmol}), \mathrm{BF}_{3} \cdot \mathrm{OEt}_{2}(73 \mathrm{ul}, 0.60 \mathrm{mmol})$ and 2,2-dimethyl 3-ethoxycyclobutanone (1.0 equiv) were used. The reaction mixture was stirred at room temperature for $10 \mathrm{~h}$ under Ar protection. After completion of the reaction, the residue was purified by silical gel column chromatography (Hexane: Ether $=10: 1)$. Finally, compound $\mathbf{4 b}(87$ $\mathrm{mg}$, yellow solid) was isolated in $83 \%$ yield. ${ }^{1} \mathrm{H}-\mathrm{NMR}\left(400 \mathrm{MHz}, \mathrm{CDCl}_{3}\right) \delta(\mathrm{ppm}) 8.16(\mathrm{~s}, 1 \mathrm{H}), 7.68$ $(\mathrm{d}, \mathrm{J}=8.8 \mathrm{~Hz}, 1 \mathrm{H}), 7.23(\mathrm{~d}, \mathrm{~J}=8.8 \mathrm{~Hz}, 1 \mathrm{H}), 4.07(\mathrm{~s}, 3 \mathrm{H}), 3.22(\mathrm{~m}, 1 \mathrm{H}), 1.36(\mathrm{~d}, 6 \mathrm{H}) ;{ }^{13} \mathrm{C}-\mathrm{NMR}(100$ $\left.\mathrm{MHz}, \mathrm{CDCl}_{3}\right) \delta(\mathrm{ppm}) 163.2,141.3,133.3,131.9,119.1,117.5,36.8,36.0,23.0 ;$ HRMS (ESI) calcd 
for $\mathrm{C}_{10} \mathrm{H}_{13} \mathrm{~N}_{3}[\mathrm{M}+\mathrm{H}]^{+}:$176.1182, found 176.1188.

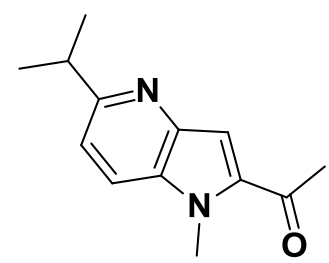

\section{4c. 1-(5-isopropyl-1-methyl-1H-pyrrolo[3,2-b]pyridin-2-yl)ethanone}

Following the general procedure II, amine $(0.30 \mathrm{mmol}), \mathrm{BF}_{3} \cdot \mathrm{OEt}_{2}(36 \mathrm{ul}, 0.60 \mathrm{mmol})$ and 2,2-dimethyl 3-ethoxycyclobutanone (1.0 equiv) were used. The reaction mixture was stirred at room temperature for $12 \mathrm{~h}$ under Ar protection. After completion of the reaction, the residue was purified by silical gel column chromatography (Hexane: Ether $=10: 1)$. Finally, compound $\mathbf{4 c}(48 \mathrm{mg}$, yellow solid) was isolated in $74 \%$ yield. ${ }^{1} \mathrm{H}-\mathrm{NMR}\left(400 \mathrm{MHz}, \mathrm{CDCl}_{3}\right) \delta(\mathrm{ppm}) 7.65(\mathrm{~d}, \mathrm{~J}=8.7 \mathrm{~Hz}, 1 \mathrm{H})$, $7.41(\mathrm{~s}, 1 \mathrm{H}), 7.21(\mathrm{~d}, \mathrm{~J}=8.7 \mathrm{~Hz}, 1 \mathrm{H}), 4.05(\mathrm{~s}, 3 \mathrm{H}), 3.20(\mathrm{~m}, 1 \mathrm{H}), 2.63(\mathrm{~s}, 3 \mathrm{H}), 1.36(\mathrm{~d}, 6 \mathrm{H}) ;{ }^{13} \mathrm{C}-\mathrm{NMR}$ $\left(100 \mathrm{MHz} \mathrm{CDCl}_{3}\right) \delta(\mathrm{ppm}) 192.2,163.3,142.8,136.5,132.5,118.9,118.5,111.6,36.8,32.4,28.3$, 23.1; HRMS (ESI) calcd for $\mathrm{C}_{13} \mathrm{H}_{16} \mathrm{~N}_{2} \mathrm{O}[\mathrm{M}+\mathrm{H}]^{+}: 217.1335$, found 217.1340.<smiles>CC(=O)c1cc2ccc(C(C)C)nc2n1C</smiles>

\section{4d. 1-(6-isopropyl-1-methyl-1H-pyrrolo[2,3-b]pyridin-2-yl)ethanone}

Following the general procedure II, amine $(0.30 \mathrm{mmol}), \mathrm{BF}_{3} \cdot \mathrm{OEt}_{2}(36 \mathrm{ul}, 0.60 \mathrm{mmol})$ and 2,2-dimethyl 3-ethoxycyclobutanone (1.0 equiv) were used. The reaction mixture was stirred at room temperature for $11 \mathrm{~h}$ under Ar protection. After completion of the reaction, the residue was purified by silical gel column chromatography (Hexane: Ether $=10: 1)$. Finally, compound 4d $(43$ $\mathrm{mg}$, primrose yellow oil) was isolated in $66 \%$ yield. ${ }^{1} \mathrm{H}-\mathrm{NMR}\left(400 \mathrm{MHz}, \mathrm{CDCl}_{3}\right) \delta(\mathrm{ppm}) 7.90(\mathrm{~d}$, $\mathrm{J}=8.4 \mathrm{~Hz}, 1 \mathrm{H}), 7.17(\mathrm{~s}, 1 \mathrm{H}), 7.03(\mathrm{~d}, \mathrm{~J}=8.4 \mathrm{~Hz}, 1 \mathrm{H}), 4.15(\mathrm{~s}, 3 \mathrm{H}), 3.16(\mathrm{~m}, 1 \mathrm{H}), 2.60(\mathrm{~s}, 3 \mathrm{H}), 1.36(\mathrm{~d}$, $6 \mathrm{H}) ;{ }^{13} \mathrm{C}-\mathrm{NMR}\left(100 \mathrm{MHz}, \mathrm{CDCl}_{3}\right) \delta$ (ppm) 191.6, 166.4, 149.5, 134.5, 131.3, 116.4, 115.2, 109.6, 37.1, 30.9, 27.7, 22.9; HRMS (ESI) calcd for $\mathrm{C}_{13} \mathrm{H}_{16} \mathrm{~N}_{2} \mathrm{O}[\mathrm{M}+\mathrm{H}]^{+}:$217.1335, found 217.1343.

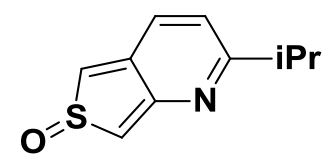

\section{4e. 2-isopropylthieno[3,4-b]pyridine}

Following the general procedure II, amine $(0.20 \mathrm{mmol}), \mathrm{BF}_{3} \cdot \mathrm{OEt}_{2}(24 \mathrm{ul}, 0.20 \mathrm{mmol})$ and 2,2-dimethyl 3-ethoxycyclobutanone (1.0 equiv) were used. The reaction mixture was stirred at room temperature for $8 \mathrm{~h}$ under Ar protection. After completion of the reaction, the residue was purified by silical gel column chromatography (Hexane: Ether $=10: 1)$. Finally, compound $\mathbf{4 e}(34 \mathrm{mg}$, yellow oil) was isolated in $87 \%$ yield. ${ }^{1} \mathrm{H}-\mathrm{NMR}\left(400 \mathrm{MHz}, \mathrm{CDCl}_{3}\right) \delta(\mathrm{ppm}) 9.26(\mathrm{~s}, 1 \mathrm{H}), 8.78(\mathrm{~d}$, $\mathrm{J}=1.2 \mathrm{~Hz}, 1 \mathrm{H}), 7.97(\mathrm{~d}, \mathrm{~J}=1.5 \mathrm{~Hz}, 1 \mathrm{H}), 7.63(\mathrm{~d}, \mathrm{~J}=5.5 \mathrm{~Hz}, 1 \mathrm{H}), 3.63(\mathrm{~m}, 1 \mathrm{H}), 1.29(\mathrm{~d}, 6 \mathrm{H}) ;{ }^{13} \mathrm{C}-\mathrm{NMR}$ $\left(100 \mathrm{MHz}_{\mathrm{CDCl}}\right) \delta(\mathrm{ppm}) 203.0,158.7,147.7,135.2$, 131.1, 125.3, 36.2, 19.2; HRMS (ESI) calcd for $\mathrm{C}_{10} \mathrm{H}_{11} \mathrm{NSO}[\mathrm{M}+\mathrm{H}]^{+}:$194.0634, found 194.0637. 
<smiles>CC(C)c1ccc2c(ccc3ccn(C)c32)n1</smiles>

\section{4f. 7-isopropyl-1-methyl-1H-pyrrolo[2,3-f]quinolone}

Following the general procedure II, amine $(0.60 \mathrm{mmol}), \mathrm{BF}_{3} \cdot \mathrm{OEt}_{2}(72 \mathrm{ul}, 0.60 \mathrm{mmol})$ and 2,2-dimethyl 3-ethoxycyclobutanone (1.0 equiv) were used. The reaction mixture was stirred at room temperature for $10 \mathrm{~h}$ under Ar protection. After completion of the reaction, the residue was purified by silical gel column chromatography (Hexane: Ether $=20: 1)$. Finally, compound $\mathbf{4 f}(102$ $\mathrm{mg}$, white solid) was isolated in $76 \%$ yield. ${ }^{1} \mathrm{H}-\mathrm{NMR}\left(400 \mathrm{MHz}, \mathrm{CDCl}_{3}\right) \delta(\mathrm{ppm}) 8.69(\mathrm{~d}, \mathrm{~J}=8.8 \mathrm{~Hz}$, $1 \mathrm{H}), 7.89(\mathrm{~d}, \mathrm{~J}=8.8 \mathrm{~Hz}, 1 \mathrm{H}), 7.75(\mathrm{~d}, \mathrm{~J}=8.8 \mathrm{~Hz}, 1 \mathrm{H}), 7.37(\mathrm{~d}, \mathrm{~J}=8.8 \mathrm{~Hz}, 1 \mathrm{H}), 7.07(\mathrm{~d}, \mathrm{~J}=2.9 \mathrm{~Hz}, 1 \mathrm{H})$, $6.61(\mathrm{~d}, \mathrm{~J}=2.9 \mathrm{~Hz}, 1 \mathrm{H}), 4.23(\mathrm{~s}, 3 \mathrm{H}), 3.29(\mathrm{~m}, 1 \mathrm{H}), 1.42(\mathrm{~d}, 6 \mathrm{H}) ;{ }^{13} \mathrm{C}-\mathrm{NMR}\left(100 \mathrm{MHz}, \mathrm{CDCl}_{3}\right) \delta$ (ppm) 164.2, 146.7, 129.6, 129.3, 129.2, 125.4, 124.4, 122.1, 117.4, 116.9, 102.4, 38.3, 37.1, 22.9; HRMS (ESI) calcd for $\mathrm{C}_{15} \mathrm{H}_{16} \mathrm{~N}_{2}[\mathrm{M}+\mathrm{H}]^{+}:$225.1386, found 225.1392<smiles>CC(C)c1ccc2c(ccc3c2ccn3C)n1</smiles>

\section{4g. 7-isopropyl-3-methyl-3H-pyrrolo[3,2-f]quinolone}

Following the general procedure II, amine $(0.30 \mathrm{mmol}), \mathrm{BF}_{3} \cdot \mathrm{OEt}_{2}(36 \mathrm{ul}, 0.30 \mathrm{mmol})$ and 2,2-dimethyl 3-ethoxycyclobutanone (1.0 equiv) were used. The reaction mixture was stirred at room temperature for $12 \mathrm{~h}$ under Ar protection. After completion of the reaction, the residue was purified by silical gel column chromatography (Hexane: Ether $=10: 1)$. Finally, compound $\mathbf{4 g}$ (48 $\mathrm{mg}$, white solid) was isolated in $71 \%$ yield. ${ }^{1} \mathrm{H}-\mathrm{NMR}\left(400 \mathrm{MHz}, \mathrm{CDCl}_{3}\right) \delta(\mathrm{ppm}) 8.46(\mathrm{~d}, \mathrm{~J}=8.5 \mathrm{~Hz}$, $1 \mathrm{H}), 7.83(\mathrm{~d}, \mathrm{~J}=9.0 \mathrm{~Hz}, 1 \mathrm{H}), 7.69(\mathrm{~d}, \mathrm{~J}=9.0 \mathrm{~Hz}, 1 \mathrm{H}), 7.39(\mathrm{~d}, \mathrm{~J}=8.5 \mathrm{~Hz}, 1 \mathrm{H}), 7.16(\mathrm{~d}, \mathrm{~J}=3.0 \mathrm{~Hz}, 1 \mathrm{H})$, $6.97(\mathrm{~d}, \mathrm{~J}=3.0 \mathrm{~Hz}, 1 \mathrm{H}), 3.92(\mathrm{~s}, 3 \mathrm{H}), 3.28(\mathrm{~m}, 1 \mathrm{H}), 1.41(\mathrm{~d}, 6 \mathrm{H}) ;{ }^{13} \mathrm{C}-\mathrm{NMR}\left(100 \mathrm{MHz}, \mathrm{CDCl}_{3}\right) \delta$ (ppm) 164.5, 144.6, 132.8, 131.7, 127.8, 123.5, 123.0, 121.7, 118.3, 114.1, 100.4, 37.3, 33.4, 23.0; HRMS (ESI) calcd for $\mathrm{C}_{15} \mathrm{H}_{16} \mathrm{~N}_{2}[\mathrm{M}+\mathrm{H}]^{+}:$225.1386, found 225.1392 .<smiles></smiles>

\section{4h. 8-isopropyl-1-methyl-1H-pyrrolo[3,2-h]quinolone}

Following the general procedure II, amine $(0.60 \mathrm{mmol}), \mathrm{BF}_{3} \cdot \mathrm{OEt}_{2}(72 \mathrm{ul}, 0.60 \mathrm{mmol})$ and 2,2-dimethyl 3-ethoxycyclobutanone (1.0 equiv) were used. The reaction mixture was stirred at room temperature for $10 \mathrm{~h}$ under Ar protection. After completion of the reaction, the residue was purified by silical gel column chromatography (Hexane: Ether $=10: 1)$. Finally, compound $\mathbf{4 h}(110$ $\mathrm{mg}$, white solid) was isolated in $82 \%$ yield. Melting point: $57-58^{\circ} \mathrm{C} .{ }^{1} \mathrm{H}-\mathrm{NMR}\left(400 \mathrm{MHz}, \mathrm{CDCl}_{3}\right) \delta$ 
(ppm) $8.12(\mathrm{~d}, \mathrm{~J}=8.3 \mathrm{~Hz}, 1 \mathrm{H}), 7.71(\mathrm{~d}, \mathrm{~J}=8.5 \mathrm{~Hz}, 1 \mathrm{H}), 7.40(\mathrm{~d}, \mathrm{~J}=8.5 \mathrm{~Hz}, 1 \mathrm{H}), 7.26(\mathrm{~d}, \mathrm{~J}=8.3 \mathrm{~Hz}, 1 \mathrm{H})$, $7.16(\mathrm{~d}, \mathrm{~J}=2.8 \mathrm{~Hz}, 1 \mathrm{H}), 6.65(\mathrm{~d}, \mathrm{~J}=2.8 \mathrm{~Hz}, 1 \mathrm{H}), 4.60(\mathrm{~s}, 3 \mathrm{H}), 3.29(\mathrm{~m}, 1 \mathrm{H}), 1.49(\mathrm{~d}, 6 \mathrm{H}) ;{ }^{13} \mathrm{C}-\mathrm{NMR}$ $\left(100 \mathrm{MHz}, \mathrm{CDCl}_{3}\right) \delta(\mathrm{ppm}) 164.5,139.7,136.2,129.9,129.5,128.0,123.7,120.9,119.3,117.3$, 102.1, 38.1, 36.9, 22.7; HRMS (ESI) calcd for $\mathrm{C}_{15} \mathrm{H}_{16} \mathrm{~N}_{2}[\mathrm{M}+\mathrm{H}]^{+}:$225.1386, found 225.1393.<smiles>CC(C)c1ccc2c(ccc3c2ccn3-c2ccccc2)n1</smiles>

\section{4i. 7-isopropyl-3-phenyl-3H-pyrrolo[3,2-f]quinolone}

Following the general procedure II, amine $(0.30 \mathrm{mmol}), \mathrm{BF}_{3} \cdot \mathrm{OEt}_{2}(36 \mathrm{ul}, 0.30 \mathrm{mmol})$ and 2,2-dimethyl 3-ethoxycyclobutanone (1.0 equiv) were used. The reaction mixture was stirred at room temperature for $9 \mathrm{~h}$ under Ar protection. After completion of the reaction, the residue was purified by silical gel column chromatography (Hexane: Ether =10:1). Finally, compound 4i (55 mg, yellow solid) was isolated in 64\% yield. ${ }^{1} \mathrm{H}-\mathrm{NMR}\left(400 \mathrm{MHz}, \mathrm{CDCl}_{3}\right) \delta(\mathrm{ppm}) 8.51(\mathrm{~d}, 1 \mathrm{H}), 7.84(\mathrm{~s}$, 1H), $7.56(\mathrm{~m}, 4 \mathrm{H}), 7.43(\mathrm{~m}, 3 \mathrm{H}), 7.16(\mathrm{~d}, 1 \mathrm{H}), 3.30(\mathrm{~m}, 1 \mathrm{H}), 1.43(\mathrm{~d}, 6 \mathrm{H}) ;{ }^{13} \mathrm{C}-\mathrm{NMR}(100 \mathrm{MHz}$, $\left.\mathrm{CDCl}_{3}\right) \delta(\mathrm{ppm}) 165.0,145.0,139.6,132.1,131.9,129.9,127.2,127.2,125.0,124.2,124.0,121.7$, 118.5, 115.2, 102.6, 37.3, 23.0; HRMS (ESI) calcd for $\mathrm{C}_{20} \mathrm{H}_{18} \mathrm{~N}_{2}[\mathrm{M}+\mathrm{H}]^{+}$: 287.1543, found 287.1550 .<smiles>CC(C)c1ccc2c(ccc3c2cnn3-c2ccccc2)n1</smiles>

\section{4j. 7-isopropyl-3-phenyl-3H-pyrazolo[4,3-f]quinolone}

Following the general procedure II, amine $(0.30 \mathrm{mmol}), \mathrm{BF}_{3} \cdot \mathrm{OEt}_{2}(36 \mathrm{ul}, 0.30 \mathrm{mmol})$ and 2,2-dimethyl 3-ethoxycyclobutanone (1.0 equiv) were used. The reaction mixture was stirred at room temperature for $9 \mathrm{~h}$ under Ar protection. After completion of the reaction, the residue was purified by silical gel column chromatography (Hexane: Ether $=10: 1)$. Finally, compound $\mathbf{4 j}$ (74 $\mathrm{mg}$, brown solid) was isolated in $86 \%$ yield. ${ }^{1} \mathrm{H}-\mathrm{NMR}\left(400 \mathrm{MHz}, \mathrm{CDCl}_{3}\right) \delta(\mathrm{ppm}) 8.57(\mathrm{~s}, 1 \mathrm{H}), 8.50(\mathrm{~d}$, $\mathrm{J}=8.4 \mathrm{~Hz}, 1 \mathrm{H}), 8.00(\mathrm{~d}, \mathrm{~J}=9.3 \mathrm{~Hz}, 1 \mathrm{H}), 7.95(\mathrm{~d}, \mathrm{~J}=9.3 \mathrm{~Hz}, 1 \mathrm{H}), 7.76(\mathrm{~d}, 2 \mathrm{H}), 7.58(\mathrm{t}, 2 \mathrm{H}), 7.49(\mathrm{~d}$, $\mathrm{J}=8.4 \mathrm{~Hz}, 1 \mathrm{H}), 7.43(\mathrm{t}, 1 \mathrm{H}), 3.30(\mathrm{~m}, 1 \mathrm{H}), 1.43(\mathrm{~d}, 6 \mathrm{H}) ;{ }^{13} \mathrm{C}-\mathrm{NMR}\left(100 \mathrm{MHz}, \mathrm{CDCl}_{3}\right) \delta(\mathrm{ppm}) 166.1$, 145.5, 139.9, 136.7, 134.1, 131.6, 129.8, 129.7, 127.5, 123.6, 120.8, 120.4, 120.0, 114.3, 37.3, 22.9; HRMS (ESI) calcd for $\mathrm{C}_{19} \mathrm{H}_{17} \mathrm{~N}_{3}[\mathrm{M}+\mathrm{H}]^{+}:$288.1495, found 288.1502. 
<smiles>CC(C)c1ccc2c(ccc3c2cnn3C)n1</smiles>

\section{4k. 7-isopropyl-3-methyl-3H-pyrazolo[4,3-f]quinolone}

Following the general procedure II, amine $(0.30 \mathrm{mmol}), \mathrm{BF}_{3} \cdot \mathrm{OEt}_{2}(36 \mathrm{ul}, 0.30 \mathrm{mmol})$ and 2,2-dimethyl 3-ethoxycyclobutanone (1.0 equiv) were used. The reaction mixture was stirred at room temperature for $9 \mathrm{~h}$ under Ar protection. After completion of the reaction, the residue was purified by silical gel column chromatography (Hexane: Ether $=10: 1)$. Finally, compound $4 \mathbf{k}(58$ $\mathrm{mg}$, white solid) was isolated in $52 \%$ yield. ${ }^{1} \mathrm{H}-\mathrm{NMR}\left(400 \mathrm{MHz}, \mathrm{CDCl}_{3}\right) \delta(\mathrm{ppm}) 8.44(\mathrm{~d}, \mathrm{~J}=8.4 \mathrm{~Hz}$, $1 \mathrm{H}), 8.35(\mathrm{~s}, 1 \mathrm{H}), 7.95(\mathrm{~d}, \mathrm{~J}=9.2 \mathrm{~Hz}, 1 \mathrm{H}), 7.67(\mathrm{~d}, \mathrm{~J}=9.2 \mathrm{~Hz}, 1 \mathrm{H}), 7.45(\mathrm{~d}, \mathrm{~J}=8.4 \mathrm{~Hz}, 1 \mathrm{H}), 4.17(\mathrm{~s}, 3 \mathrm{H})$, $3.27(\mathrm{~m}, 1 \mathrm{H}), 1.41(\mathrm{~d}, 6 \mathrm{H}) ;{ }^{13} \mathrm{C}-\mathrm{NMR}\left(100 \mathrm{MHz}, \mathrm{CDCl}_{3}\right) \delta(\mathrm{ppm}) 165.6,145.2,137.6,131.8,131.5$, 129.2, 120.9, 119.8, 119.0, 113.1, 37.2, 36.1, 22.9; HRMS (ESI) calcd for $\mathrm{C}_{14} \mathrm{H}_{15} \mathrm{~N}_{3}[\mathrm{M}+\mathrm{H}]^{+}$: 226.1339 , found 226.1346 .<smiles>CC(C)c1ccc2c(ccc3nn(C)cc32)n1</smiles>

\section{4l. 7-isopropyl-2-methyl-2H-pyrazolo[4,3-f]quinolone}

Following the general procedure II, amine $(0.30 \mathrm{mmol}), \mathrm{BF}_{3} \cdot \mathrm{OEt}_{2}(36 \mathrm{ul}, 0.30 \mathrm{mmol})$ and 2,2-dimethyl 3-ethoxycyclobutanone (1.0 equiv) were used. The reaction mixture was stirred at room temperature for $10 \mathrm{~h}$ under Ar protection. After completion of the reaction, the residue was purified by silical gel column chromatography (Hexane: Ether =10:1). Finally, compound $4 \mathbf{l}$ (54 mg, yellow solid) was isolated in $79 \%$ yield. ${ }^{1} \mathrm{H}-\mathrm{NMR}\left(400 \mathrm{MHz}, \mathrm{CDCl}_{3}\right) \delta(\mathrm{ppm}) 8.23(\mathrm{~d}, \mathrm{~J}=8.4 \mathrm{~Hz}, 1 \mathrm{H})$, $8.17(\mathrm{~s}, 1 \mathrm{H}), 7.83(\mathrm{~d}, \mathrm{~J}=9.2 \mathrm{~Hz}, 1 \mathrm{H}), 7.78(\mathrm{~d}, \mathrm{~J}=9.2 \mathrm{~Hz}, 1 \mathrm{H}), 7.33$ (d, J=8.4Hz, 1H), 4.20 (s, 3H), 3.24 $(\mathrm{m}, 1 \mathrm{H}), 1.39(\mathrm{~d}, 6 \mathrm{H}) ;{ }^{13} \mathrm{C}-\mathrm{NMR}\left(100 \mathrm{MHz}, \mathrm{CDCl}_{3}\right) \delta(\mathrm{ppm})$ 165.5, 147.2, 146.7, 131.6, 129.4, 123.7, 121.1, 120.8, 118.8, 118.0, 40.3, 37.0, 22.9; HRMS (ESI) calcd for $\mathrm{C}_{14} \mathrm{H}_{15} \mathrm{~N}_{3}[\mathrm{M}+\mathrm{H}]^{+}$: 226.1339 , found 226.1346 .<smiles>CC(C)c1ccc2c(ccc3cn(C)nc32)n1</smiles>

\section{4m. 7-isopropyl-2-methyl-2H-pyrazolo[3,4-f]quinolone}

Following the general procedure II, amine $(0.30 \mathrm{mmol}), \mathrm{BF}_{3} \cdot \mathrm{OEt}_{2}(36 \mathrm{ul}, 0.30 \mathrm{mmol})$ and 2,2-dimethyl 3-ethoxycyclobutanone (1.0 equiv) were used. The reaction mixture was stirred at room temperature for $10 \mathrm{~h}$ under Ar protection. After completion of the reaction, the residue was purified by silical gel column chromatography (Hexane: Ether $=10: 1)$. Finally, compound $\mathbf{4 m}(52$ $\mathrm{mg}$, yellow solid) was isolated in $77 \%$ yield. Melting point: $81-82^{\circ} \mathrm{C} .{ }^{1} \mathrm{H}-\mathrm{NMR}\left(400 \mathrm{MHz}, \mathrm{CDCl}_{3}\right) \delta$ 
(ppm) $8.75(\mathrm{~d}, \mathrm{~J}=8.4 \mathrm{~Hz}, 1 \mathrm{H}), 7.89(\mathrm{~s}, 1 \mathrm{H}), 7.72(\mathrm{~d}, \mathrm{~J}=9.2 \mathrm{~Hz}, 1 \mathrm{H}), 7.69(\mathrm{~d}, \mathrm{~J}=9.2 \mathrm{~Hz}, 1 \mathrm{H}), 7.41(\mathrm{~d}$, $\mathrm{J}=8.4 \mathrm{~Hz}, 1 \mathrm{H}), 4.25(\mathrm{~s}, 3 \mathrm{H}), 3.26(\mathrm{~m}, 1 \mathrm{H}), 1.41(\mathrm{~d}, 6 \mathrm{H}) ;{ }^{13} \mathrm{C}-\mathrm{NMR}\left(100 \mathrm{MHz}, \mathrm{CDCl}_{3}\right) \delta(\mathrm{ppm})$ 167.0, 148.6, 145.8, 130.7, 125.0, 124.9, 121.7, 119.1, 118.7, 118.6, 40.2, 37.2, 22.9; IR (ATR) 2955, 2863, 1614, 1590, 1560, 1469, 1431, 1399, 1373, 1355, 1331, 1289, 1251, 1209, 1158, 1129, 1103, 1081, 1026, 995, 976, 926, 879, 848, 826, 779, 728, 681, $659 \mathrm{~cm}^{-1}$; HRMS (ESI) calcd for $\mathrm{C}_{14} \mathrm{H}_{15} \mathrm{~N}_{3}$ $[\mathrm{M}+\mathrm{H}]^{+}:$226.1339, found 226.1346<smiles>CC(C)c1ccc2c(ccc3cnn(C)c32)n1</smiles>

\section{4n. 7-isopropyl-1-methyl-1H-pyrazolo[3,4-f]quinolone}

Following the general procedure II, amine $(0.30 \mathrm{mmol}), \mathrm{BF}_{3} \cdot \mathrm{OEt}_{2}(36 \mathrm{ul}, 0.30 \mathrm{mmol})$ and 2,2-dimethyl 3-ethoxycyclobutanone (1.0 equiv) were used. The reaction mixture was stirred at room temperature for $12 \mathrm{~h}$ under Ar protection. After completion of the reaction, the residue was purified by silical gel column chromatography (Hexane: Ether $=10: 1)$. Finally, compound 4n (34 $\mathrm{mg}$, pale yellow oil) was isolated in $50 \%$ yield. ${ }^{1} \mathrm{H}-\mathrm{NMR}\left(400 \mathrm{MHz}, \mathrm{CDCl}_{3}\right) \delta$ (ppm) 8.69 (d, $\mathrm{J}=8.6 \mathrm{~Hz}, 1 \mathrm{H}), 8.02(\mathrm{~s}, 1 \mathrm{H}), 7.88(\mathrm{~d}, \mathrm{~J}=8.7 \mathrm{~Hz}, 1 \mathrm{H}), 7.73(\mathrm{~d}, \mathrm{~J}=8.7 \mathrm{~Hz}, 1 \mathrm{H}), 7.47(\mathrm{~d}, \mathrm{~J}=8.6 \mathrm{~Hz}, 1 \mathrm{H})$, $4.50(\mathrm{~s}, 3 \mathrm{H}), 3.30(\mathrm{~m}, 1 \mathrm{H}), 1.42(\mathrm{~d}, 6 \mathrm{H}) ;{ }^{13} \mathrm{C}-\mathrm{NMR}\left(100 \mathrm{MHz}, \mathrm{CDCl}_{3}\right) \delta(\mathrm{ppm}) 166.9,148.8,135.3$, 133.3, 130.2, 124.0, 122.8, 121.2, 118.5, 115.0, 40.6, 37.2, 22.8; HRMS (ESI) calcd for $\mathrm{C}_{14} \mathrm{H}_{15} \mathrm{~N}_{3}$ $[\mathrm{M}+\mathrm{H}]^{+}:$226.1339, found 226.1344.<smiles>CC(C)c1ccc2c(ccc3cnn(-c4ccccc4)c32)c1</smiles>

\section{4o-1. 7-isopropyl-1-phenyl-1H-pyrazolo[3,4-f]quinolone}

Following the general procedure II, amine $(0.30 \mathrm{mmol}), \mathrm{BF}_{3} \cdot \mathrm{OEt}_{2}(36 \mathrm{ul}, 0.30 \mathrm{mmol})$ and 2,2-dimethyl 3-ethoxycyclobutanone (1.0 equiv) were used. The reaction mixture was stirred at room temperature for $12 \mathrm{~h}$ under Ar protection. After completion of the reaction, the residue was purified by silical gel column chromatography (Hexane: Ether $=10: 1)$. Finally, compound 4o-1 (55 $\mathrm{mg}$, pale yellow solid) was isolated in $64 \%$ yield. Melting point: $79-80^{\circ} \mathrm{C}$. ${ }^{1} \mathrm{H}-\mathrm{NMR}(400 \mathrm{MHz}$, $\left.\mathrm{CDCl}_{3}\right) \delta(\mathrm{ppm}) 8.23(\mathrm{~s}, 1 \mathrm{H}), 7.94(\mathrm{~d}, \mathrm{~J}=8.9 \mathrm{~Hz}, 1 \mathrm{H}), 7.85(\mathrm{~d}, \mathrm{~J}=8.9 \mathrm{~Hz}, 1 \mathrm{H}), 7.79(\mathrm{~d}, \mathrm{~J}=8.9 \mathrm{~Hz}, 1 \mathrm{H})$, $7.59(\mathrm{~m}, 5 \mathrm{H}), 7.16(\mathrm{~d}, \mathrm{~J}=8.9 \mathrm{~Hz}, 1 \mathrm{H}), 3.23(\mathrm{~m}, 1 \mathrm{H}), 1.36(\mathrm{~d}, 6 \mathrm{H}) ;{ }^{13} \mathrm{C}-\mathrm{NMR}\left(100 \mathrm{MHz}, \mathrm{CDCl}_{3}\right) \delta$ (ppm) 167.2, 148.8, 141.6, 135.7, 135.6, 130.3, 129.8, 129.4, 127.1, 124.7, 122.4, 121.3, 118.1, 114.5, 37.2, 22.7; IR (ATR) 3065, 2966, 2923, 2867, 1612, 1596, 1580, 1500, 1453, 1403, 1361, 1314, 1297, 1206, 1183, 1145, 1087, 1071, 1018, 999, 960, 922, 879, 841, 831, 769, $720 \mathrm{~cm}^{-1}$; HRMS (ESI) calcd for $\mathrm{C}_{19} \mathrm{H}_{17} \mathrm{~N}_{3}[\mathrm{M}+\mathrm{H}]^{+}: 288.1495$, found 288.1503 .<smiles>CC(C)c1ccc2cc3cnn(-c4ccccc4)c3cc2n1</smiles> 


\section{4o-2. 7-isopropyl-1-phenyl-1H-pyrazolo[4,3-g]quinolone}

Following the general procedure II, amine $(0.30 \mathrm{mmol}), \mathrm{BF}_{3} \cdot \mathrm{OEt}_{2}(36 \mathrm{ul}, 0.30 \mathrm{mmol})$ and 2,2-dimethyl 3-ethoxycyclobutanone (1.0 equiv) were used. The reaction mixture was stirred at room temperature for $12 \mathrm{~h}$ under Ar protection. After completion of the reaction, the residue was purified by silical gel column chromatography (Hexane: Ether $=10: 1)$. Finally, compound 40-2 (14 $\mathrm{mg}$, colorless oil) was isolated in $16 \%$ yield. ${ }^{1} \mathrm{H}-\mathrm{NMR}\left(400 \mathrm{MHz}, \mathrm{CDCl}_{3}\right) \delta(\mathrm{ppm}) 8.47(\mathrm{~s}, 1 \mathrm{H}), 8.44$ $(\mathrm{s}, 1 \mathrm{H}), 8.27(\mathrm{~s}, 1 \mathrm{H}), 8.24(\mathrm{~d}, 1 \mathrm{H}), 7.88(\mathrm{~d}, 2 \mathrm{H}), 7.56(\mathrm{t}, 2 \mathrm{H}), 7.36(\mathrm{t}, 1 \mathrm{H}), 7.28(\mathrm{~d}, 1 \mathrm{H}), 3.27(\mathrm{~m}, 1 \mathrm{H})$, $1.42(\mathrm{~d}, 6 \mathrm{H}) ;{ }^{13} \mathrm{C}-\mathrm{NMR}\left(100 \mathrm{MHz}, \mathrm{CDCl}_{3}\right) \delta(\mathrm{ppm}) 169.2,146.4,140.5,139.3,137.7,136.1,129.6$, $126.5,125.9,122.9,122.5,119.8,117.9,107.2,37.8,22.4$; HRMS (ESI) calcd for $\mathrm{C}_{19} \mathrm{H}_{17} \mathrm{~N}_{3}[\mathrm{M}+\mathrm{H}]^{+}$: 288.1495, found 288.1502 .

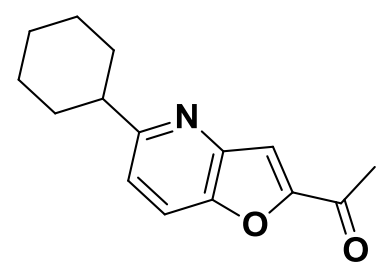

\section{5a. 1-(5-isopropylfuro[3,2-b]pyridin-2-yl)ethanone}

Following the general procedure II, amine $(0.30 \mathrm{mmol}), \mathrm{BF}_{3} \cdot \mathrm{OEt}_{2}(36 \mathrm{ul}, 0.30 \mathrm{mmol})$ and substituted 3-ethoxycyclobutanone (1.0 equiv) were used. The reaction mixture was stirred at room temperature for $10 \mathrm{~h}$ under Ar protection. After completion of the reaction, the residue was purified by silical gel column chromatography (Hexane: Ether $=10: 1)$. Finally, compound 5a $(50 \mathrm{mg}$, colorless oil) was isolated in $69 \%$ yield. ${ }^{1} \mathrm{H}-\mathrm{NMR}\left(400 \mathrm{MHz}, \mathrm{CDCl}_{3}\right) \delta(\mathrm{ppm}) 8.00(\mathrm{~d}, \mathrm{~J}=8.8 \mathrm{~Hz}, 1 \mathrm{H})$, $7.46(\mathrm{~s}, 1 \mathrm{H}), 7.23(\mathrm{~d}, \mathrm{~J}=8.8 \mathrm{~Hz}, 1 \mathrm{H}), 2.84(\mathrm{t}, 1 \mathrm{H}), 2.67(\mathrm{~s}, 3 \mathrm{H}), 2.00(\mathrm{~d}, 2 \mathrm{H}), 1.91(\mathrm{~d}, 2 \mathrm{H}), 1.78(\mathrm{~m}$, $1 \mathrm{H}), 1.67(\mathrm{q}, 2 \mathrm{H}), 1.41(\mathrm{q}, 2 \mathrm{H}), 1.36(\mathrm{~m}, 1 \mathrm{H}) ;{ }^{13} \mathrm{C}-\mathrm{NMR}\left(100 \mathrm{MHz}, \mathrm{CDCl}_{3}\right) \delta(\mathrm{ppm}) 189.6,167.1$, $161.9,151.7,132.8,118.8,117.1,110.3,47.0,33.2,26.8,26.5,26.0$; HRMS (ESI) calcd for $\mathrm{C}_{15} \mathrm{H}_{17} \mathrm{NO}_{2}[\mathrm{M}+\mathrm{H}]^{+}:$244.1332, found 244.1336.

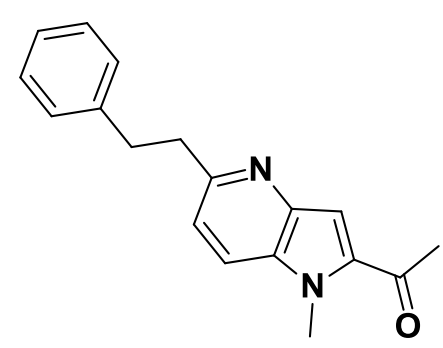

\section{5b. 1-(1-methyl-5-phenethyl-1H-pyrrolo[3,2-b]pyridin-2-yl)ethanone}

Following the general procedure II, amine $(0.20 \mathrm{mmol}), \mathrm{BF}_{3} \cdot \mathrm{OEt}_{2}(24 \mathrm{ul}, 0.20 \mathrm{mmol})$ and substituted 3-ethoxycyclobutanone (1.0 equiv) were used. The reaction mixture was stirred at room temperature for $12 \mathrm{~h}$ under Ar protection. After completion of the reaction, the residue was purified by silical gel column chromatography (Hexane: Ether $=10: 1)$. Finally, compound $\mathbf{5 b}(45$ $\mathrm{mg}$, colorless oil) was isolated in $81 \%$ yield. ${ }^{1} \mathrm{H}-\mathrm{NMR}\left(400 \mathrm{MHz}, \mathrm{CDCl}_{3}\right) \delta$ (ppm) 7.61 (d, $\mathrm{J}=8.4 \mathrm{~Hz}, 1 \mathrm{H}), 7.41(\mathrm{~s}, 1 \mathrm{H}), 7.26(\mathrm{~m}, 5 \mathrm{H}), 7.09(\mathrm{~d}, \mathrm{~J}=8.4 \mathrm{~Hz}, 1 \mathrm{H}), 4.05(\mathrm{~s}, 3 \mathrm{H}), 3.22(\mathrm{~m}, 2 \mathrm{H}), 3.11(\mathrm{~m}$, 2H), 2.65 (s, 3H); ${ }^{13} \mathrm{C}-\mathrm{NMR}\left(100 \mathrm{MHz}, \mathrm{CDCl}_{3}\right) \delta(\mathrm{ppm})$ 192.2, 157.1, 143.1, 141.8, 136.6, 132.3, $128.7,128.5,126.1,120.6,118.7,111.4,40.6,36.7,32.5,28.3$; HRMS (ESI) calcd for $\mathrm{C}_{18} \mathrm{H}_{18} \mathrm{~N}_{2} \mathrm{O}$ $[\mathrm{M}+\mathrm{H}]^{+}:$279.1492, found 279.1498 . 


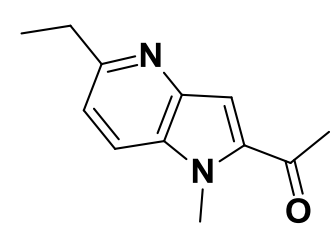

\section{5c. 1-(5-ethyl-1-methyl-1H-pyrrolo[3,2-b]pyridin-2-yl)ethanone}

Following the general procedure II, amine $(0.30 \mathrm{mmol}), \mathrm{BF}_{3} \cdot \mathrm{OEt}_{2}(36 \mathrm{ul}, 0.30 \mathrm{mmol})$ and substituted 3-ethoxycyclobutanone (1.0 equiv) were used. The reaction mixture was stirred at room temperature for $12 \mathrm{~h}$ under Ar protection. After completion of the reaction, the residue was purified by silical gel column chromatography (Hexane: Ether $=20: 1)$. Finally, compound $\mathbf{5 c}(52 \mathrm{mg}$, white solid) was isolated in $86 \%$ yield. Melting point: $95-96^{\circ} \mathrm{C} .{ }^{1} \mathrm{H}-\mathrm{NMR}\left(400 \mathrm{MHz}, \mathrm{CDCl}_{3}\right) \delta(\mathrm{ppm}) 7.63$ $(\mathrm{d}, \mathrm{J}=8.7 \mathrm{~Hz}, 1 \mathrm{H}), 7.36(\mathrm{~s}, 1 \mathrm{H}), 7.17(\mathrm{~d}, \mathrm{~J}=8.7 \mathrm{~Hz}, 1 \mathrm{H}), 4.04(\mathrm{~s}, 3 \mathrm{H}), 2.93(\mathrm{q}, 2 \mathrm{H}), 2.62(\mathrm{~s}, 3 \mathrm{H}), 1.35(\mathrm{t}$, $3 \mathrm{H}) ;{ }^{13} \mathrm{C}-\mathrm{NMR}\left(100 \mathrm{MHz}, \mathrm{CDCl}_{3}\right) \delta$ (ppm) 192.2, 159.4, 143.0, 136.5, 132.3, 119.8, 118.8, 111.4, 32.4, 31.8, 28.3, 14.6; IR (ATR) 3047, 2966, 2921, 2852, 1913, 1672, 1565, 1505, 1467, 1441, 1417, 1394, 1354, 1327, 1300, 1214, 1196, 1170, 1151, 1103, 1062, 1018, 981, 964, 932, 822, 801, 781, $752,686 \mathrm{~cm}^{-1}$; HRMS (ESI) calcd for $\mathrm{C}_{12} \mathrm{H}_{14} \mathrm{~N}_{2} \mathrm{O}[\mathrm{M}+\mathrm{H}]^{+}:$203.1179, found 203.1186.

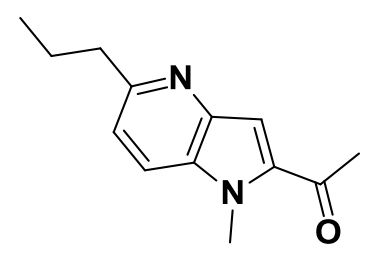

\section{5d. 1-(1-methyl-5-propyl-1H-pyrrolo[3,2-b]pyridin-2-yl)ethanone}

Following the general procedure II, amine $(0.20 \mathrm{mmol}), \mathrm{BF}_{3} \cdot \mathrm{OEt}_{2}(24 \mathrm{ul}, 0.20 \mathrm{mmol})$ and substituted 3-ethoxycyclobutanone (1.0 equiv) were used. The reaction mixture was stirred at room temperature for $12 \mathrm{~h}$ under Ar protection. After completion of the reaction, the residue was purified by silical gel column chromatography (Hexane: Ether $=20: 1)$. Finally, compound $\mathbf{5 d}(29 \mathrm{mg}$, yellow oil) was isolated in $67 \%$ yield. ${ }^{1} \mathrm{H}-\mathrm{NMR}\left(400 \mathrm{MHz}, \mathrm{CDCl}_{3}\right) \delta(\mathrm{ppm}) 7.62(\mathrm{~d}, \mathrm{~J}=8.7 \mathrm{~Hz}, 1 \mathrm{H}), 7.37(\mathrm{~s}$, $1 \mathrm{H}), 7.16(\mathrm{~d}, \mathrm{~J}=8.7 \mathrm{~Hz}, 1 \mathrm{H}), 4.04(\mathrm{~s}, 3 \mathrm{H}), 2.87(\mathrm{t}, 2 \mathrm{H}), 2.63(\mathrm{~s}, 3 \mathrm{H}), 1.80(\mathrm{~m}, 2 \mathrm{H}), 1.35(\mathrm{t}, 3 \mathrm{H})$; ${ }^{13} \mathrm{C}-\mathrm{NMR}\left(100 \mathrm{MHz}, \mathrm{CDCl}_{3}\right) \delta$ (ppm) 192.2, 158.2, 143.0, 136.5, 132.3, 120.4, 118.6, 111.4, 40.8, 32.4, 28.3, 23.8, 14.0; HRMS (ESI) calcd for $\mathrm{C}_{13} \mathrm{H}_{16} \mathrm{~N}_{2} \mathrm{O}[\mathrm{M}+\mathrm{H}]^{+}:$: 217.1335, found 217.1342.

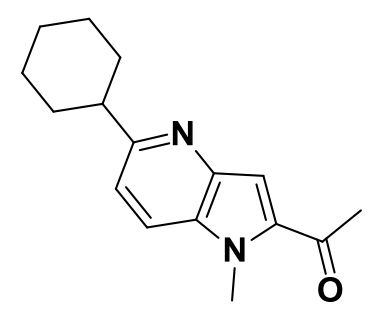

\section{5e. 1-(5-cyclohexyl-1-methyl-1H-pyrrolo[3,2-b]pyridin-2-yl)ethanone}

Following the general procedure II, amine $(0.20 \mathrm{mmol}), \mathrm{BF}_{3} \cdot \mathrm{OEt}_{2}(24 \mathrm{ul}, 0.20 \mathrm{mmol})$ and substituted 3-ethoxycyclobutanone (1.0 equiv) were used. The reaction mixture was stirred at room temperature for $10 \mathrm{~h}$ under Ar protection. After completion of the reaction, the residue was purified by silical gel column chromatography (Hexane: Ether $=15: 1)$. Finally, compound $\mathbf{5 e}(40 \mathrm{mg}$, write solid) was isolated in $78 \%$ yield. ${ }^{1} \mathrm{H}-\mathrm{NMR}\left(400 \mathrm{MHz}, \mathrm{CDCl}_{3}\right) \delta(\mathrm{ppm}) 7.64(\mathrm{~d}, \mathrm{~J}=8.7 \mathrm{~Hz}, 1 \mathrm{H}), 7.40$ 
(s, 1H), $7.18(\mathrm{~d}, \mathrm{~J}=8.7 \mathrm{~Hz}, 1 \mathrm{H}), 4.04(\mathrm{~s}, 3 \mathrm{H}), 2.84(\mathrm{~m}, 1 \mathrm{H}), 2.63(\mathrm{~s}, 3 \mathrm{H}), 1.98(\mathrm{~d}, 2 \mathrm{H}), 1.87(\mathrm{~d}, 2 \mathrm{H})$, $1.77(\mathrm{~d}, 1 \mathrm{H}), 1.58$ (q, 2H), 1.44 (q, 2H), $1.30(\mathrm{~m}, 1 \mathrm{H}) ;{ }^{13} \mathrm{C}-\mathrm{NMR}\left(100 \mathrm{MHz}, \mathrm{CDCl}_{3}\right) \delta(\mathrm{ppm})$ 192.2, 162.4, 142.8, 136.5, 132.5, 118.9, 118.8, 111.6, 47.1, 33.5, 32.4, 28.3, 26.8, 26.3; HRMS (ESI) calcd for $\mathrm{C}_{16} \mathrm{H}_{20} \mathrm{~N}_{2} \mathrm{O}[\mathrm{M}+\mathrm{H}]^{+}:$257.1648, found 257.1656.

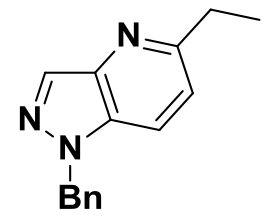

\section{5f. 1-benzyl-5-ethyl-1H-pyrazolo[4,3-b]pyridine}

Following the general procedure II, amine $(0.30 \mathrm{mmol}), \mathrm{BF}_{3} \cdot \mathrm{OEt}_{2}(36 \mathrm{ul}, 0.30 \mathrm{mmol})$ and substituted 3-ethoxycyclobutanone (1.0 equiv) were used. The reaction mixture was stirred at room temperature for $10 \mathrm{~h}$ under Ar protection. After completion of the reaction, the residue was purified by silical gel column chromatography (Hexane: Ether =10:1). Finally, compound $\mathbf{5 f}(62 \mathrm{mg}$, pale yellow oil) was isolated in $87 \%$ yield. ${ }^{1} \mathrm{H}-\mathrm{NMR}\left(400 \mathrm{MHz}, \mathrm{CDCl}_{3}\right) \delta(\mathrm{ppm}) 8.20(\mathrm{~s}, 1 \mathrm{H}), 7.55(\mathrm{~d}$, $\mathrm{J}=8.8 \mathrm{~Hz}, 1 \mathrm{H}), 7.28(\mathrm{~m}, 3 \mathrm{H}), 7.19(\mathrm{~d}, 2 \mathrm{H}), 7.12(\mathrm{~d}, \mathrm{~J}=8.8 \mathrm{~Hz}, 1 \mathrm{H}), 5.58(\mathrm{~s}, 2 \mathrm{H}), 2.94(\mathrm{q}, 2 \mathrm{H}), 1.35$ (t, $3 \mathrm{H}) ;{ }^{13} \mathrm{C}-\mathrm{NMR}\left(100 \mathrm{MHz}, \mathrm{CDCl}_{3}\right) \delta$ (ppm) 159.6, 142.0, 136.5, 133.8, 131.5, 129.0, 128.2, 127.4, 120.6, 117.9, 53.9, 31.7, 14.4; HRMS (ESI) calcd for $\mathrm{C}_{15} \mathrm{H}_{15} \mathrm{~N}_{3}[\mathrm{M}+\mathrm{H}]^{+}:$: 238.1339, found 238.1348.

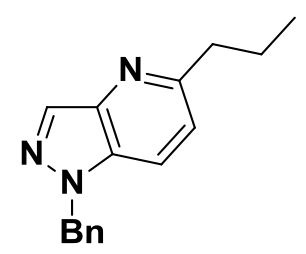

\section{5g. 1-benzyl-5-propyl-1H-pyrazolo[4,3-b]pyridine}

Following the general procedure II, amine $(0.20 \mathrm{mmol}), \mathrm{BF}_{3} \cdot \mathrm{OEt}_{2}(36 \mathrm{ul}, 0.20 \mathrm{mmol})$ and substituted 3-ethoxycyclobutanone (1.0 equiv) were used. The reaction mixture was stirred at room temperature for $10 \mathrm{~h}$ under Ar protection. After completion of the reaction, the residue was purified by silical gel column chromatography (Hexane: Ether $=10: 1)$. Finally, compound 5g (29mg, brown sloid) was isolated in 58\% yield. ${ }^{1} \mathrm{H}-\mathrm{NMR}\left(400 \mathrm{MHz}, \mathrm{CDCl}_{3}\right) \delta(\mathrm{ppm}) 8.18(\mathrm{~s}, 1 \mathrm{H}), 7.52(\mathrm{~d}$, $\mathrm{J}=8.8 \mathrm{~Hz}, 1 \mathrm{H}), 7.28(\mathrm{~m}, 3 \mathrm{H}), 7.16(\mathrm{~d}, 2 \mathrm{H}), 7.08(\mathrm{~d}, \mathrm{~J}=8.8 \mathrm{~Hz}, 1 \mathrm{H}), 5.55(\mathrm{~s}, 2 \mathrm{H}), 2.85(\mathrm{t}, 2 \mathrm{H}), 1.76(\mathrm{~m}$, 2H), $0.96(\mathrm{t}, 3 \mathrm{H}) ;{ }^{13} \mathrm{C}-\mathrm{NMR}\left(100 \mathrm{MHz}, \mathrm{CDCl}_{3}\right) \delta(\mathrm{ppm}) 158.4,142.0,136.5,133.7,131.5,129.0$, 128.2, 127.4, 121.1, 117.7, 53.9, 40.6, 23.7, 14.1; HRMS (ESI) calcd for $\mathrm{C}_{16} \mathrm{H}_{17} \mathrm{~N}_{3}[\mathrm{M}+\mathrm{H}]^{+}$: 252.1495, found 252.1501.

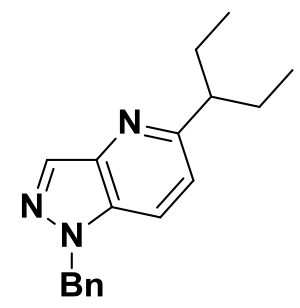

\section{5h. 1-benzyl-5-(pentan-3-yl)-1H-pyrazolo[4,3-b]pyridine}

Following the general procedure II, amine $(0.20 \mathrm{mmol}), \mathrm{BF}_{3} \cdot \mathrm{OEt}_{2}(36 \mathrm{ul}, 0.20 \mathrm{mmol})$ and substituted 3-ethoxycyclobutanone (1.0 equiv) were used. The reaction mixture was stirred at room 
temperature for $12 \mathrm{~h}$ under Ar protection. After completion of the reaction, the residue was purified by silical gel column chromatography (Hexane: Ether $=15: 1)$. Finally, compound $\mathbf{5 h}$ (43mg, pale yellow oil) was isolated in $77 \%$ yield. ${ }^{1} \mathrm{H}-\mathrm{NMR}\left(400 \mathrm{MHz}, \mathrm{CDCl}_{3}\right) \delta(\mathrm{ppm}) 8.23(\mathrm{~s}, 1 \mathrm{H}), 7.55(\mathrm{~d}$, $\mathrm{J}=8.8 \mathrm{~Hz}, 1 \mathrm{H}), 7.28(\mathrm{~m}, 3 \mathrm{H}), 7.22(\mathrm{~d}, 2 \mathrm{H}), 7.07(\mathrm{~d}, \mathrm{~J}=8.8 \mathrm{~Hz}, 1 \mathrm{H}), 5.57(\mathrm{~s}, 2 \mathrm{H}), 2.69(\mathrm{~m}, 1 \mathrm{H}), 1.74(\mathrm{~m}$, $4 \mathrm{H}), 0.78(\mathrm{t}, 6 \mathrm{H}) ;{ }^{13} \mathrm{C}-\mathrm{NMR}\left(100 \mathrm{MHz}, \mathrm{CDCl}_{3}\right) \delta(\mathrm{ppm})$ 161.5, 141.9, 136.4, 133.8, 131.6, 129.0, 128.2, 127.5, 120.4, 117.6, 53.8, 51.8, 28.7, 12.3; HRMS (ESI) calcd for $\mathrm{C}_{18} \mathrm{H}_{21} \mathrm{~N}_{3}[\mathrm{M}+\mathrm{H}]^{+}$: 280.1808 , found 280.1816 .

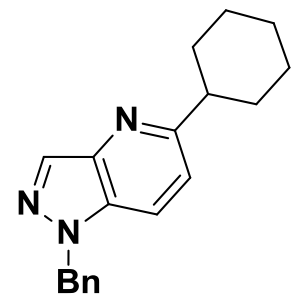

\section{5i. 1-benzyl-5-cyclohexyl-1H-pyrazolo[4,3-b]pyridine}

Following the general procedure $\mathbf{I I}$, amine $(0.20 \mathrm{mmol}), \mathrm{BF}_{3} \cdot \mathrm{OEt}_{2}(24 \mathrm{ul}, 0.20 \mathrm{mmol})$ and substituted 3-ethoxycyclobutanone (1.0 equiv) were used. The reaction mixture was stirred at room temperature for $11 \mathrm{~h}$ under Ar protection. After completion of the reaction, the residue was purified by silical gel column chromatography (Hexane: Ether $=15: 1)$. Finally, compound 5i $(47 \mathrm{mg}$, write solid) was isolated in $80 \%$ yield. Melting point: $78-79^{\circ} \mathrm{C} .{ }^{1} \mathrm{H}-\mathrm{NMR}\left(400 \mathrm{MHz}, \mathrm{CDCl}_{3}\right) \delta(\mathrm{ppm}) 8.22$ $(\mathrm{s}, 1 \mathrm{H}), 7.55(\mathrm{~d}, \mathrm{~J}=8.8 \mathrm{~Hz}, 1 \mathrm{H}), 7.28(\mathrm{~m}, 3 \mathrm{H}), 7.19(\mathrm{~d}, 2 \mathrm{H}), 7.12(\mathrm{~d}, \mathrm{~J}=8.8 \mathrm{~Hz}, 1 \mathrm{H}), 5.56(\mathrm{~s}, 2 \mathrm{H}), 2.83$ $(\mathrm{m}, 1 \mathrm{H}), 1.97(\mathrm{~d}, 2 \mathrm{H}), 1.88(\mathrm{~d}, 2 \mathrm{H}), 1.75(\mathrm{~m}, 1 \mathrm{H}), 1.58(\mathrm{q}, 2 \mathrm{H}), 1.44(\mathrm{q}, 2 \mathrm{H}), 1.40(\mathrm{~m}, 1 \mathrm{H})$; ${ }^{13} \mathrm{C}-\mathrm{NMR}\left(100 \mathrm{MHz}, \mathrm{CDCl}_{3}\right) \delta$ (ppm) 162.6, 141.7, 136.4, 133.8, 131.5, 129.0, 128.1, 127.4, 119.7, 117.8, 53.8, 47.0, 33.3, 26.7, 26.2; IR (ATR) 3061, 3028, 2926, 2856, 2114, 1774, 1604, 1568, 1493, 1452, 1428, 1409, 1377, 1328, 1301, 1261, 1176, 1157, 1133, 1103, 1076, 1049, 1028, 949, 892, 868, 847, 819, 798, 776, 744, 723, $694 \mathrm{~cm}^{-1}$; HRMS (ESI) calcd for $\mathrm{C}_{19} \mathrm{H}_{21} \mathrm{~N}_{3}[\mathrm{M}+\mathrm{H}]^{+}:$292.1808, found 292.1815 .<smiles>Cn1ccc2ccc3ccc(Cc4ccccc4)nc3c21</smiles>

\section{5j. 8-benzyl-1-methyl-1H-pyrrolo[3,2-h]quinoline}

Following the general procedure $\mathbf{I I}$, amine $(0.30 \mathrm{mmol}), \mathrm{BF}_{3} \cdot \mathrm{OEt}_{2}(36 \mathrm{ul}, 0.30 \mathrm{mmol})$ and substituted 3-ethoxycyclobutanone (1.0 equiv) were used. The reaction mixture was stirred at room temperature for $9 \mathrm{~h}$ under Ar protection. After completion of the reaction, the residue was purified by silical gel column chromatography (Hexane: Ether $=10: 1$ ). Finally, compound 5j (73mg, yellow solid) was isolated in $90 \%$ yield. ${ }^{1} \mathrm{H}-\mathrm{NMR}\left(400 \mathrm{MHz}, \mathrm{CDCl}_{3}\right) \delta(\mathrm{ppm}) 8.76(\mathrm{~d}, 1 \mathrm{H}), 7.72(\mathrm{~d}$, $\mathrm{J}=8.8 \mathrm{~Hz}, 1 \mathrm{H}), 7.33(\mathrm{~d}, \mathrm{~J}=8.8 \mathrm{~Hz}, 1 \mathrm{H}), 7.31(\mathrm{~m}, 5 \mathrm{H}), 7.25(\mathrm{~m}, 1 \mathrm{H}), 7.10(\mathrm{~d}, \mathrm{~J}=2.8 \mathrm{~Hz}, 1 \mathrm{H}), 6.61(\mathrm{~d}$, $\mathrm{J}=2.8 \mathrm{~Hz}, 1 \mathrm{H}), 4.53(\mathrm{~s}, 3 \mathrm{H}), 4.49(\mathrm{~s}, 2 \mathrm{H}) ;{ }^{13} \mathrm{C}-\mathrm{NMR}\left(100 \mathrm{MHz}, \mathrm{CDCl}_{3}\right) \delta(\mathrm{ppm}) 147.3,146.1,139.5$, 130.1, 129.4, 129.0, 128.7, 128.5, 127.8, 126.5, 124.4, 121.8, 120.0, 119.2, 115.4, 102.0, 39.2, 38.2; HRMS (ESI) calcd for $\mathrm{C}_{19} \mathrm{H}_{16} \mathrm{~N}_{2}[\mathrm{M}+\mathrm{H}]^{+}:$273.1386, found 273.1392 . 
<smiles>Cc1ccc2ccc3ccn(C)c3nc1-2</smiles>

\section{5k. 1,8-dimethyl-1H-pyrrolo[3,2-h]quinoline}

Following the general procedure II, amine $(0.30 \mathrm{mmol}), \mathrm{BF}_{3} \cdot \mathrm{OEt}_{2}(36 \mathrm{ul}, 0.30 \mathrm{mmol})$ and substituted 3-ethoxycyclobutanone (1.0 equiv) were used. The reaction mixture was stirred at room temperature for $8 \mathrm{~h}$ under Ar protection. After completion of the reaction, the residue was purified by silical gel column chromatography (Hexane: Ether $=10: 1)$. Finally, compound $\mathbf{5 k}(54 \mathrm{mg}$, colorless oil) was isolated in $92 \%$ yield. ${ }^{1} \mathrm{H}-\mathrm{NMR}\left(400 \mathrm{MHz}, \mathrm{CDCl}_{3}\right) \delta(\mathrm{ppm}) 8.06(\mathrm{~d}, \mathrm{~J}=8 \mathrm{~Hz}, 1 \mathrm{H}), 7.66(\mathrm{~d}$, $\mathrm{J}=8.8 \mathrm{~Hz}, 1 \mathrm{H}), 7.35(\mathrm{~d}, \mathrm{~J}=8.8 \mathrm{~Hz}, 1 \mathrm{H}), 7.20(\mathrm{~d}, \mathrm{~J}=8 \mathrm{~Hz}, 1 \mathrm{H}), 7.13(\mathrm{~d}, \mathrm{~J}=2.8 \mathrm{~Hz}, 1 \mathrm{H}), 6.61(\mathrm{~d}, \mathrm{~J}=2.8 \mathrm{~Hz}$, $1 \mathrm{H}), 4.55$ (s, 3H), 2.74 (s, 3H); ${ }^{13} \mathrm{C}-\mathrm{NMR}\left(100 \mathrm{MHz}, \mathrm{CDCl}_{3}\right) \delta(\mathrm{ppm})$ 156.0, 147.1, 139.9, 136.2, 129.6, 128.0, 123.4, 120.8, 119.3, 119.1, 102.1, 37.9, 25.5; HRMS (ESI) calcd for $\mathrm{C}_{13} \mathrm{H}_{12} \mathrm{~N}_{2}[\mathrm{M}+\mathrm{H}]^{+}$: 197.1073, found 197.1080.<smiles>Cn1ccc2ccc3ccc(Cc4ccccc4)nc3c21</smiles>

\section{5l. 1-methyl-8-phenethyl-1H-pyrrolo[3,2-h]quinoline}

Following the general procedure II, amine $(0.30 \mathrm{mmol}), \mathrm{BF}_{3} \cdot \mathrm{OEt}_{2}(36 \mathrm{ul}, 0.30 \mathrm{mmol})$ and substituted 3-ethoxycyclobutanone (1.0 equiv) were used. The reaction mixture was stirred at room temperature for $8 \mathrm{~h}$ under Ar protection. After completion of the reaction, the residue was purified by silical gel column chromatography (Hexane: Ether $=10: 1)$. Finally, compound 5l (75mg, yellow solid) was isolated in $88 \%$ yield. Melting point: $80-81^{\circ} \mathrm{C} .{ }^{1} \mathrm{H}-\mathrm{NMR}\left(400 \mathrm{MHz}, \mathrm{CDCl}_{3}\right) \delta(\mathrm{ppm}) 8.09$ $(\mathrm{d}, \mathrm{J}=8 \mathrm{~Hz}, 1 \mathrm{H}), 7.72(\mathrm{~d}, \mathrm{~J}=8.5 \mathrm{~Hz}, 1 \mathrm{H}), 7.40(\mathrm{~d}, \mathrm{~J}=8.5 \mathrm{~Hz}, 1 \mathrm{H}), 7.31(\mathrm{~m}, 4 \mathrm{H}), 7.25(\mathrm{~m}, 1 \mathrm{H}), 7.19(\mathrm{~d}$, $\mathrm{J}=8.5 \mathrm{~Hz}, 1 \mathrm{H}), 7.18(\mathrm{~d}, \mathrm{~J}=2.8 \mathrm{~Hz}, 1 \mathrm{H}), 6.66(\mathrm{~d}, \mathrm{~J}=2.8 \mathrm{~Hz}, 1 \mathrm{H}), 4.56(\mathrm{~s}, 3 \mathrm{H}), 3.35(\mathrm{~m}, 4 \mathrm{H}) ;{ }^{13} \mathrm{C}-\mathrm{NMR}$ $\left(100 \mathrm{MHz}, \mathrm{CDCl}_{3}\right) \delta(\mathrm{ppm}) 158.6,142.3,139.9,136.2,129.7,129.6,128.6,128.5,128.0,126.0$, 123.7, 121.0, 119.3, 118.8, 102.2, 40.5, 38.0, 35.3; HRMS (ESI) calcd for $\mathrm{C}_{20} \mathrm{H}_{18} \mathrm{~N}_{2}[\mathrm{M}+\mathrm{H}]^{+}$: 287.1543 , found 287.1549 .<smiles></smiles>

\section{5m. 8-ethyl-1-methyl-1H-pyrrolo[3,2-h]quinolone}

Following the general procedure II, amine $(0.30 \mathrm{mmol}), \mathrm{BF}_{3} \cdot \mathrm{OEt}_{2}(36 \mathrm{ul}, 0.30 \mathrm{mmol})$ and substituted 3-ethoxycyclobutanone (1.0 equiv) were used. The reaction mixture was stirred at room temperature for $11 \mathrm{~h}$ under Ar protection. After completion of the reaction, the residue was purified by silical gel column chromatography (Hexane: Ether $=10: 1$ ). Finally, compound $\mathbf{5 m}$ (pale yellow 
oil) was isolated in 95\% yield. ${ }^{1} \mathrm{H}-\mathrm{NMR}\left(400 \mathrm{MHz}, \mathrm{CDCl}_{3}\right) \delta(\mathrm{ppm}) 8.09(\mathrm{~d}, \mathrm{~J}=8.3 \mathrm{~Hz}, 1 \mathrm{H}), 7.69(\mathrm{~d}$, $\mathrm{J}=8.5 \mathrm{~Hz}, 1 \mathrm{H}), 7.38(\mathrm{~d}, \mathrm{~J}=8.5 \mathrm{~Hz}, 1 \mathrm{H}), 7.22(\mathrm{~d}, \mathrm{~J}=8.3 \mathrm{~Hz}, 1 \mathrm{H}), 7.15(\mathrm{~d}, \mathrm{~J}=2.8 \mathrm{~Hz}, 1 \mathrm{H}), 6.63(\mathrm{~d}, \mathrm{~J}=$ $2.8 \mathrm{~Hz}, 1 \mathrm{H}), 4.58(\mathrm{~s}, 3 \mathrm{H}), 3.05(\mathrm{q}, 2 \mathrm{H}), 1.48(\mathrm{t}, 3 \mathrm{H}) ;{ }^{13} \mathrm{C}-\mathrm{NMR}\left(100 \mathrm{MHz}, \mathrm{CDCl}_{3}\right) \delta(\mathrm{ppm}) 160.7$, 139.9, 136.1, 129.8, 129.5, 128.0, 123.6, 120.8, 119.3, 118.3, 102.1, 38.0, 32.0, 13.4; HRMS (ESI) calcd for $\mathrm{C}_{14} \mathrm{H}_{14} \mathrm{~N}_{2}[\mathrm{M}+\mathrm{H}]^{+}:$211.1230, found 211.1237.<smiles>Cn1ccc2ccc3ccc(C4CCCCC4)nc3c21</smiles>

\section{5n. 8-cyclohexyl-1-methyl-1H-pyrrolo[3,2-h]quinolone}

Following the general procedure II, amine $(0.30 \mathrm{mmol}), \mathrm{BF}_{3} \cdot \mathrm{OEt}_{2}(36 \mathrm{ul}, 0.30 \mathrm{mmol})$ and substituted 3-ethoxycyclobutanone (1.0 equiv) were used. The reaction mixture was stirred at room temperature for $12 \mathrm{~h}$ under Ar protection. After completion of the reaction, the residue was purified by silical gel column chromatography (Hexane: Ether $=10: 1)$. Finally, compound 5n $(74 \mathrm{mg}$, pale yellow solid) was isolated in $93 \%$ yield. Melting point: $126-127^{\circ} \mathrm{C} .{ }^{1} \mathrm{H}-\mathrm{NMR}\left(400 \mathrm{MHz}, \mathrm{CDCl}_{3}\right) \delta$ (ppm) $8.09(\mathrm{~d}, \mathrm{~J}=8.3 \mathrm{~Hz}, 1 \mathrm{H}), 7.69(\mathrm{~d}, \mathrm{~J}=8.5 \mathrm{~Hz}, 1 \mathrm{H}), 7.37(\mathrm{~d}, \mathrm{~J}=8.5 \mathrm{~Hz}, 1 \mathrm{H}), 7.22(\mathrm{~d}, \mathrm{~J}=8.3 \mathrm{~Hz}, 1 \mathrm{H})$, $7.15(\mathrm{~d}, \mathrm{~J}=2.8 \mathrm{~Hz}, 1 \mathrm{H}), 6.62(\mathrm{~d}, \mathrm{~J}=2.8 \mathrm{~Hz}, 1 \mathrm{H}), 4.58(\mathrm{~s}, 3 \mathrm{H}), 2.91(\mathrm{t}, 1 \mathrm{H}), 2.11(\mathrm{~d}, 2 \mathrm{H}), 1.95(\mathrm{~d}, 2 \mathrm{H})$, $1.84(\mathrm{~m}, 1 \mathrm{H}), 1.70(\mathrm{q}, 2 \mathrm{H}), 1.50(\mathrm{q}, 2 \mathrm{H}), 1.38(\mathrm{~m}, 1 \mathrm{H}) ;{ }^{13} \mathrm{C}-\mathrm{NMR}\left(100 \mathrm{MHz}, \mathrm{CDCl}_{3}\right) \delta(\mathrm{ppm}) 163.8$, 139.8, 136.1, 129.9, 129.4, 127.9, 123.7, 120.8, 119.3, 117.6, 102.1, 47.0, 38.1, 33.1, 26.9, 26.5; IR (ATR) 3102, 2918, 2872, 2848, 2106, 1707, 1931, 1607, 1595, 1541, 1524, 1501, 1461, 1447, 1416, 1400, 1364, 1348, 1327, 1291, 1261, 1238, 1208, 1184, 1146, 1129, 1071, 1056, 999, 959, 904, 890, $867,853,834,801,792,756,743,714,687 \mathrm{~cm}^{-1}$; HRMS (ESI) calcd for $\mathrm{C}_{18} \mathrm{H}_{20} \mathrm{~N}_{2}[\mathrm{M}+\mathrm{H}]^{+}$: 265.1699 , found 265.1705 .<smiles>CCC(CC)c1ccc2ccc3ccn(C)c3n12</smiles>

\section{5o. 1-methyl-8-(pentan-3-yl)-1H-pyrrolo[3,2-h]quinolone}

Following the general procedure II, amine $(0.30 \mathrm{mmol}), \mathrm{BF}_{3} \cdot \mathrm{OEt}_{2}(36 \mathrm{ul}, 0.30 \mathrm{mmol})$ and substituted 3-ethoxycyclobutanone (1.0 equiv) were used. The reaction mixture was stirred at room temperature for $12 \mathrm{~h}$ under Ar protection. After completion of the reaction, the residue was purified by silical gel column chromatography (Hexane: Ether $=10: 1)$. Finally, compound 5o $(71 \mathrm{mg}$, brown solid) was isolated in $94 \%$ yield. ${ }^{1} \mathrm{H}-\mathrm{NMR}\left(400 \mathrm{MHz}, \mathrm{CDCl}_{3}\right) \delta(\mathrm{ppm}) 8.09(\mathrm{~d}, \mathrm{~J}=8.3 \mathrm{~Hz}, 1 \mathrm{H}), 7.69$ $(\mathrm{d}, \mathrm{J}=8.5 \mathrm{~Hz}, 1 \mathrm{H}), 7.39$ (d, J=8.5Hz, 1H), $7.18(\mathrm{~d}, \mathrm{~J}=8.3 \mathrm{~Hz}, 1 \mathrm{H}), 7.15(\mathrm{~d}, \mathrm{~J}=2.8 \mathrm{~Hz}, 1 \mathrm{H}), 6.63(\mathrm{~d}, \mathrm{~J}=$ $2.8 \mathrm{~Hz}, 1 \mathrm{H}), 4.56(\mathrm{~s}, 3 \mathrm{H}), 2.76(\mathrm{~m}, 1 \mathrm{H}), 1.94(\mathrm{~m}, 2 \mathrm{H}), 1.82(\mathrm{~m}, 2 \mathrm{H}), 0.86(\mathrm{t}, 6 \mathrm{H}) ;{ }^{13} \mathrm{C}-\mathrm{NMR}(100$ $\left.\mathrm{MHz}, \mathrm{CDCl}_{3}\right) \delta(\mathrm{ppm}) 162.6,140.1,135.8,129.9,129.4,127.8,123.8,120.8,119.3,102.1,51.9$, 38.1, 28.5, 12.3; HRMS (ESI) calcd for $\mathrm{C}_{17} \mathrm{H}_{20} \mathrm{~N}_{2}[\mathrm{M}+\mathrm{H}]^{+}: 253.1699$, found 253.1707. 
<smiles></smiles>

\section{5p. 1-methyl-8-(pentan-3-yl)-1H-pyrrolo[3,2-h]quinolone}

Following the general procedure II, amine $(0.30 \mathrm{mmol}), \mathrm{BF}_{3} \cdot \mathrm{OEt}_{2}(36 \mathrm{ul}, 0.30 \mathrm{mmol})$ and substituted 3-ethoxycyclobutanone (1.0 equiv) were used. The reaction mixture was stirred at room temperature for $10 \mathrm{~h}$ under Ar protection. After completion of the reaction, the residue was purified by silical gel column chromatography (Hexane: Ether $=10: 1)$. Finally, compound 5p (62mg, pale yellow oil) was isolated in 92\% yield. ${ }^{1} \mathrm{H}-\mathrm{NMR}\left(400 \mathrm{MHz}, \mathrm{CDCl}_{3}\right) \delta(\mathrm{ppm}) 8.08(\mathrm{~d}, \mathrm{~J}=8.3 \mathrm{~Hz}, 1 \mathrm{H})$, $7.67(\mathrm{~d}, \mathrm{~J}=8.5 \mathrm{~Hz}, 1 \mathrm{H}), 7.37(\mathrm{~d}, \mathrm{~J}=8.5 \mathrm{~Hz}, 1 \mathrm{H}), 7.20(\mathrm{~d}, \mathrm{~J}=8.3 \mathrm{~Hz}, 1 \mathrm{H}), 7.14(\mathrm{~d}, \mathrm{~J}=2.8 \mathrm{~Hz}, 1 \mathrm{H}), 6.62(\mathrm{~d}$, $\mathrm{J}=2.8 \mathrm{~Hz}, 1 \mathrm{H}), 4.56(\mathrm{~s}, 3 \mathrm{H}), 2.99(\mathrm{t}, 2 \mathrm{H}), 1.95(\mathrm{q}, 2 \mathrm{H}), 1.05(\mathrm{t}, 3 \mathrm{H}) ;{ }^{13} \mathrm{C}-\mathrm{NMR}\left(100 \mathrm{MHz}, \mathrm{CDCl}_{3}\right) \delta$ (ppm) 159.7, 139.9, 136.1, 129.9, 129.5, 128.0, 123.6, 120.8, 119.3, 118.8, 102.1, 41.0, 38.0, 22.5, 14.1; HRMS (ESI) calcd for $\mathrm{C}_{15} \mathrm{H}_{16} \mathrm{~N}_{2}[\mathrm{M}+\mathrm{H}]^{+}:$:225.1386, found 225.1394 .<smiles>CCCc1ccc2c(ccc3ccn(C)c32)n1</smiles>

\section{5q. 1-methyl-7-propyl-1H-pyrrolo[2,3-f]quinoline}

Following the general procedure II, amine $(0.20 \mathrm{mmol}), \mathrm{BF}_{3} \cdot \mathrm{OEt}_{2}(24 \mathrm{ul}, 0.20 \mathrm{mmol})$ and substituted 3-ethoxycyclobutanone (1.0 equiv) were used. The reaction mixture was stirred at room temperature for $12 \mathrm{~h}$ under Ar protection. After completion of the reaction, the residue was purified by silical gel column chromatography (Hexane: Ether $=20: 1)$. Finally, compound 5q (38mg, white solid) was isolated in $84 \%$ yield. ${ }^{1} \mathrm{H}-\mathrm{NMR}\left(400 \mathrm{MHz}, \mathrm{CDCl}_{3}\right) \delta(\mathrm{ppm}) 8.68(\mathrm{~d}, \mathrm{~J}=8.4 \mathrm{~Hz}, 1 \mathrm{H}), 7.89$ $(\mathrm{d}, \mathrm{J}=8.8 \mathrm{~Hz}, 1 \mathrm{H}), 7.74(\mathrm{~d}, \mathrm{~J}=8.8 \mathrm{~Hz}, 1 \mathrm{H}), 7.33(\mathrm{~d}, \mathrm{~J}=8.4 \mathrm{~Hz}, 1 \mathrm{H}), 7.09(\mathrm{~d}, \mathrm{~J}=2.9 \mathrm{~Hz}, 1 \mathrm{H}), 6.62(\mathrm{~d}, \mathrm{~J}=$ $2.9 \mathrm{~Hz}, 1 \mathrm{H}), 4.25(\mathrm{~s}, 3 \mathrm{H}), 2.97(\mathrm{t}, 2 \mathrm{H}), 1.87(\mathrm{q}, 2 \mathrm{H}), 1.03(\mathrm{t}, 3 \mathrm{H}) ;{ }^{13} \mathrm{C}-\mathrm{NMR}\left(100 \mathrm{MHz}, \mathrm{CDCl}_{3}\right) \delta$ (ppm) 159.1, 146.6, 129.5, 128.8, 125.3, 124.4, 121.8, 119.7, 116.6, 102.3, 41.0, 38.2, 23.5, 14.1; HRMS (ESI) calcd for $\mathrm{C}_{15} \mathrm{H}_{16} \mathrm{~N}_{2}[\mathrm{M}+\mathrm{H}]^{+}:$225.1386, found 225.1392.<smiles>CCC(CC)c1ccc2c(ccc3ccn(C)c32)n1</smiles>

\section{5r. 1-methyl-7-(pentan-3-yl)-1H-pyrrolo[2,3-f]quinoline}

Following the general procedure II, amine $(0.20 \mathrm{mmol}), \mathrm{BF}_{3} \cdot \mathrm{OEt}_{2}(24 \mathrm{ul}, 0.20 \mathrm{mmol})$ and substituted 3-ethoxycyclobutanone (1.0 equiv) were used. The reaction mixture was stirred at room temperature for $12 \mathrm{~h}$ under Ar protection. After completion of the reaction, the residue was purified by silical gel column chromatography (Hexane: Ether $=20: 1$ ). Finally, compound 5r (37mg, colorless oil) was isolated in $73 \%$ yield. ${ }^{1} \mathrm{H}-\mathrm{NMR}\left(400 \mathrm{MHz}, \mathrm{CDCl}_{3}\right) \delta(\mathrm{ppm}) 8.68(\mathrm{~d}, \mathrm{~J}=8.8 \mathrm{~Hz}, 1 \mathrm{H})$, $7.90(\mathrm{~d}, \mathrm{~J}=8.4 \mathrm{~Hz}, 1 \mathrm{H}), 7.77$ (d, J=8.5Hz, 1H), 7.29 (d, J=8.8Hz, 1H), 7.07 (d, J=2.8Hz, 1H), 6.61 (d, 
$\mathrm{J}=2.8 \mathrm{~Hz}, 1 \mathrm{H}), 4.23(\mathrm{~s}, 3 \mathrm{H}), 2.80(\mathrm{~m}, 1 \mathrm{H}), 1.82(\mathrm{~m}, 4 \mathrm{H}), 0.85(\mathrm{t}, 6 \mathrm{H}) ;{ }^{13} \mathrm{C}-\mathrm{NMR}\left(100 \mathrm{MHz}, \mathrm{CDCl}_{3}\right)$ $\delta(\mathrm{ppm}) 162.6,140.1,135.8,129.9,129.4,127.8,123.8,120.8,119.3,102.1,51.9,38.1,28.5,12.3$; HRMS (ESI) calcd for $\mathrm{C}_{17} \mathrm{H}_{20} \mathrm{~N}_{2}[\mathrm{M}+\mathrm{H}]^{+}:$253.1699, found 253.1703.<smiles>Cn1ccc2ccc3nc(C4CCCCC4)ccc3c21</smiles>

\section{5s. 7-cyclohexyl-1-methyl-1H-pyrrolo[2,3-f]quinoline}

Following the general procedure II, amine $(0.30 \mathrm{mmol}), \mathrm{BF}_{3} \cdot \mathrm{OEt}_{2}(36 \mathrm{ul}, 0.30 \mathrm{mmol})$ and substituted 3-ethoxycyclobutanone (1.0 equiv) were used. The reaction mixture was stirred at room temperature for $12 \mathrm{~h}$ under Ar protection. After completion of the reaction, the residue was purified by silical gel column chromatography (Hexane: Ether $=20: 1)$. Finally, compound $\mathbf{5 s}(62 \mathrm{mg}$, white solid) was isolated in $78 \%$ yield. Melting point: $113-114^{\circ} \mathrm{C} .{ }^{1} \mathrm{H}-\mathrm{NMR}\left(400 \mathrm{MHz}, \mathrm{CDCl}_{3}\right) \delta(\mathrm{ppm})$ $8.67(\mathrm{~d}, \mathrm{~J}=8.8 \mathrm{~Hz}, 1 \mathrm{H}), 7.89(\mathrm{~d}, \mathrm{~J}=9.2 \mathrm{~Hz}, 1 \mathrm{H}), 7.75(\mathrm{~d}, \mathrm{~J}=9.2 \mathrm{~Hz}, 1 \mathrm{H}), 7.34(\mathrm{~d}, \mathrm{~J}=8.8 \mathrm{~Hz}, 1 \mathrm{H}), 7.07$ (d, $\mathrm{J}=2.8 \mathrm{~Hz}, 1 \mathrm{H}), 6.61(\mathrm{~d}, \mathrm{~J}=2.8 \mathrm{~Hz}, 1 \mathrm{H}), 4.22(\mathrm{~s}, 3 \mathrm{H}), 2.94(\mathrm{~m}, 1 \mathrm{H}), 2.08(\mathrm{~d}, 2 \mathrm{H}), 1.92(\mathrm{~d}, 2 \mathrm{H}), 1.85$ $(\mathrm{m}, 1 \mathrm{H}), 1.67(\mathrm{q}, 2 \mathrm{H}), 1.54(\mathrm{q}, 2 \mathrm{H}), 1.38(\mathrm{~m}, 1 \mathrm{H}) ;{ }^{13} \mathrm{C}-\mathrm{NMR}\left(100 \mathrm{MHz}, \mathrm{CDCl}_{3}\right) \delta(\mathrm{ppm}) 163.3$, 146.7, 129.6, 129.2, 129.1, 125.3, 124.4, 122.0, 117.9, 116.9, 102.4, 47.4, 38.3, 33.2, 26.8, 26.3; HRMS (ESI) calcd for $\mathrm{C}_{18} \mathrm{H}_{20} \mathrm{~N}_{2}[\mathrm{M}+\mathrm{H}]^{+}:$265.1699, found 265.1705.<smiles>Cn1ncc2c3ccc(C4CCCCC4)nc3ccc21</smiles>

\section{5t. 7-cyclohexyl-3-methyl-3H-pyrazolo[4,3-f]quinolone}

Following the general procedure II, amine $(0.30 \mathrm{mmol}), \mathrm{BF}_{3} \cdot \mathrm{OEt}_{2}(36 \mathrm{ul}, 0.30 \mathrm{mmol})$ and substituted 3-ethoxycyclobutanone (1.0 equiv) were used. The reaction mixture was stirred at room temperature for $12 \mathrm{~h}$ under Ar protection. After completion of the reaction, the residue was purified by silical gel column chromatography (Hexane: Ether $=10: 1)$. Finally, compound $5 \mathrm{t}$ (59mg, yellow oil) was isolated in 74\% yield. ${ }^{1} \mathrm{H}-\mathrm{NMR}\left(400 \mathrm{MHz}, \mathrm{CDCl}_{3}\right) \delta(\mathrm{ppm}) 8.44(\mathrm{~d}, \mathrm{~J}=8.4 \mathrm{~Hz}, 1 \mathrm{H}), 8.34(\mathrm{~s}$, $1 \mathrm{H}), 7.95(\mathrm{~d}, \mathrm{~J}=9.2 \mathrm{~Hz}, 1 \mathrm{H}), 7.67(\mathrm{~d}, \mathrm{~J}=9.2 \mathrm{~Hz}, 1 \mathrm{H}), 7.44(\mathrm{~d}, \mathrm{~J}=8.4 \mathrm{~Hz}, 1 \mathrm{H}), 4.17(\mathrm{~s}, 3 \mathrm{H}), 2.93(\mathrm{t}, 1 \mathrm{H})$, $2.04(\mathrm{~d}, 2 \mathrm{H}), 1.95(\mathrm{~d}, 2 \mathrm{H}), 1.84(\mathrm{~m}, 1 \mathrm{H}), 1.70(\mathrm{q}, 2 \mathrm{H}), 1.50(\mathrm{q}, 2 \mathrm{H}), 1.35(\mathrm{~m}, 1 \mathrm{H}) ;{ }^{13} \mathrm{C}-\mathrm{NMR}(100$ $\left.\mathrm{MHz}, \mathrm{CDCl}_{3}\right) \delta(\mathrm{ppm}) 164.8,145.2,137.5,131.8,131.5,129.1,120.9,120.2,119.0,113.1,47.5$, 36.1, 33.3, 26.8, 26.3; HRMS (ESI) calcd for $\mathrm{C}_{17} \mathrm{H}_{19} \mathrm{~N}_{3}[\mathrm{M}+\mathrm{H}]^{+}:$266.1652, found 266.1657.<smiles>CCc1ccc2c(ccc3c2cnn3C)n1</smiles> 


\section{5u. 7-ethyl-3-methyl-3H-pyrazolo[4,3-f]quinolone}

Following the general procedure II, amine $(0.30 \mathrm{mmol}), \mathrm{BF}_{3} \cdot \mathrm{OEt}_{2}(36 \mathrm{ul}, 0.30 \mathrm{mmol})$ and substituted 3-ethoxycyclobutanone (1.0 equiv) were used. The reaction mixture was stirred at room temperature for $12 \mathrm{~h}$ under Ar protection. After completion of the reaction, the residue was purified by silical gel column chromatography (Hexane: Ether $=10: 1)$. Finally, compound 5u (42mg, brown solid) was isolated in $66 \%$ yield. ${ }^{1} \mathrm{H}-\mathrm{NMR}\left(400 \mathrm{MHz}, \mathrm{CDCl}_{3}\right) \delta(\mathrm{ppm}) 8.42(\mathrm{~d}, 1 \mathrm{H}), 8.34(\mathrm{~s}, 1 \mathrm{H})$, $7.94(\mathrm{~d}, 1 \mathrm{H}), 7.67(\mathrm{~d}, 1 \mathrm{H}), 7.42(\mathrm{~d}, 1 \mathrm{H}), 4.17(\mathrm{~s}, 3 \mathrm{H}), 3.02(\mathrm{q}, 2 \mathrm{H}), 1.41(\mathrm{t}, 3 \mathrm{H}) ;{ }^{13} \mathrm{C}-\mathrm{NMR}(100 \mathrm{MHz}$, $\left.\mathrm{CDCl}_{3}\right) \delta(\mathrm{ppm}) 161.8,145.4,137.5,131.8,131.5,129.0,121.5,120.7,119.0,113.2,36.1,32.2,14.4$; HRMS (ESI) calcd for $\mathrm{C}_{13} \mathrm{H}_{13} \mathrm{~N}_{3}[\mathrm{M}+\mathrm{H}]^{+}:$212.1182, found 212.1188.

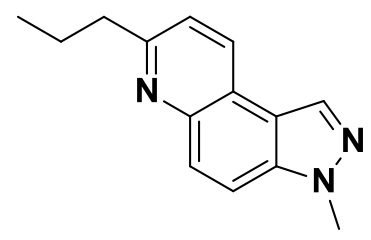

\section{5v. 3-methyl-7-propyl-3H-pyrazolo[4,3-f]quinolone}

Following the general procedure II, amine $(0.30 \mathrm{mmol}), \mathrm{BF}_{3} \cdot \mathrm{OEt}_{2}(36 \mathrm{ul}, 0.30 \mathrm{mmol})$ and substituted 3-ethoxycyclobutanone (1.0 equiv) were used. The reaction mixture was stirred at room temperature for $12 \mathrm{~h}$ under Ar protection. After completion of the reaction, the residue was purified by silical gel column chromatography (Hexane: Ether $=10: 1)$. Finally, compound 5v (51mg, colorless oil) was isolated in $75 \%$ yield. ${ }^{1} \mathrm{H}-\mathrm{NMR}\left(400 \mathrm{MHz}, \mathrm{CDCl}_{3}\right) \delta(\mathrm{ppm}) 8.42(\mathrm{~d}, \mathrm{~J}=8.4 \mathrm{~Hz}, 1 \mathrm{H})$, $8.34(\mathrm{~s}, 1 \mathrm{H}), 7.96(\mathrm{~d}, \mathrm{~J}=9.2 \mathrm{~Hz}, 1 \mathrm{H}), 7.67(\mathrm{~d}, \mathrm{~J}=9.2 \mathrm{~Hz}, 1 \mathrm{H}), 7.41(\mathrm{~d}, \mathrm{~J}=8.4 \mathrm{~Hz}, 1 \mathrm{H}), 4.16$ (s, 3H), 2.97 $(\mathrm{t}, 2 \mathrm{H}), 1.86(\mathrm{~m}, 2 \mathrm{H}), 1.03(\mathrm{t}, 3 \mathrm{H}) ;{ }^{13} \mathrm{C}-\mathrm{NMR}\left(100 \mathrm{MHz}, \mathrm{CDCl}_{3}\right) \delta(\mathrm{ppm}) 160.6,145.3,137.5,131.8$, 131.4, 128.9, 122.1, 120.8, 119.0, 113.3, 41.1, 36.1, 23.6, 14.1; HRMS (ESI) calcd for $\mathrm{C}_{14} \mathrm{H}_{15} \mathrm{~N}_{3}$ $[\mathrm{M}+\mathrm{H}]^{+}:$226.1339, found 226.1345 .<smiles>CCC(CC)c1ccc2c(ccc3c2cnn3C)n1</smiles>

5w. 3-methyl-7-(pentan-3-yl)-3H-pyrazolo[4,3-f]quinolone

Following the general procedure II, amine $(0.30 \mathrm{mmol}), \mathrm{BF}_{3} \cdot \mathrm{OEt}_{2}(36 \mathrm{ul}, 0.30 \mathrm{mmol})$ and substituted 3-ethoxycyclobutanone (1.0 equiv) were used. The reaction mixture was stirred at room temperature for $12 \mathrm{~h}$ under Ar protection. After completion of the reaction, the residue was purified by silical gel column chromatography (Hexane: Ether $=10: 1$ ). Finally, compound 5w (45mg, yellow oil) was isolated in 59\% yield. ${ }^{1} \mathrm{H}-\mathrm{NMR}\left(400 \mathrm{MHz}, \mathrm{CDCl}_{3}\right) \delta(\mathrm{ppm}) 8.43(\mathrm{~d}, \mathrm{~J}=8.4 \mathrm{~Hz}, 1 \mathrm{H}), 8.36(\mathrm{~s}$, $1 \mathrm{H}), 7.98(\mathrm{~d}, \mathrm{~J}=9.2 \mathrm{~Hz}, 1 \mathrm{H}), 7.68(\mathrm{~d}, \mathrm{~J}=9.2 \mathrm{~Hz}, 1 \mathrm{H}), 7.38(\mathrm{~d}, \mathrm{~J}=8.4 \mathrm{~Hz}, 1 \mathrm{H}), 4.18(\mathrm{~s}, 3 \mathrm{H}), 2.79(\mathrm{~m}, 1 \mathrm{H})$, $1.81(\mathrm{~m}, 4 \mathrm{H}), 0.83(\mathrm{t}, 6 \mathrm{H}) ;{ }^{13} \mathrm{C}-\mathrm{NMR}\left(100 \mathrm{MHz}, \mathrm{CDCl}_{3}\right) \delta(\mathrm{ppm}) 163.8,145.4,144.3,137.6,131.8$, 131.2, 129.3, 121.1, 119.0, 113.0, 52.2, 36.1, 28.5, 12.3; HRMS (ESI) calcd for $\mathrm{C}_{16} \mathrm{H}_{19} \mathrm{~N}_{3}[\mathrm{M}+\mathrm{H}]^{+}$: 254.1652, found 254.1659. 


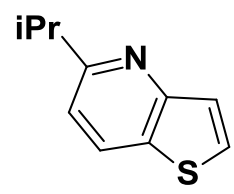

\section{5x. 5-isopropylthieno[3,2-b]pyridine}

Following the general procedure II, amine $(0.30 \mathrm{mmol}), \mathrm{BF}_{3} \cdot \mathrm{OEt}_{2}(36 \mathrm{ul}, 0.30 \mathrm{mmol})$ and substituted 3-ethoxycyclobutanone (1.0 equiv) were used. The reaction mixture was stirred at room temperature for $12 \mathrm{~h}$ under Ar protection. After completion of the reaction, the residue was purified by silical gel column chromatography (Hexane: Ether $=20: 1)$. Finally, compound 5x (47mg, pale yellow solid) was isolated in $89 \%$ yield. ${ }^{1} \mathrm{H}-\mathrm{NMR}\left(400 \mathrm{MHz}, \mathrm{CDCl}_{3}\right) \delta(\mathrm{ppm}) 8.11(\mathrm{~d}, \mathrm{~J}=8.4 \mathrm{~Hz}, 1 \mathrm{H})$, $7.70(\mathrm{~d}, \mathrm{~J}=5.2 \mathrm{~Hz}, 1 \mathrm{H}), 7.53(\mathrm{~d}, \mathrm{~J}=5.2 \mathrm{~Hz}, 1 \mathrm{H}), 7.18(\mathrm{~d}, \mathrm{~J}=8.4 \mathrm{~Hz}, 1 \mathrm{H}), 3.2(\mathrm{~m}, 1 \mathrm{H}), 1.37(\mathrm{~d}, 6 \mathrm{H})$; ${ }^{13} \mathrm{C}-\mathrm{NMR}\left(100 \mathrm{MHz}, \mathrm{CDCl}_{3}\right) \delta$ (ppm) 165.4, 155.7, 130.9, 130.8, 130.3, 125.2, 116.5, 36.8, 23.1; HRMS (ESI) calcd for $\mathrm{C}_{10} \mathrm{H}_{11} \mathrm{NS}[\mathrm{M}+\mathrm{H}]^{+}:$178.0685, found 178.0691 . 


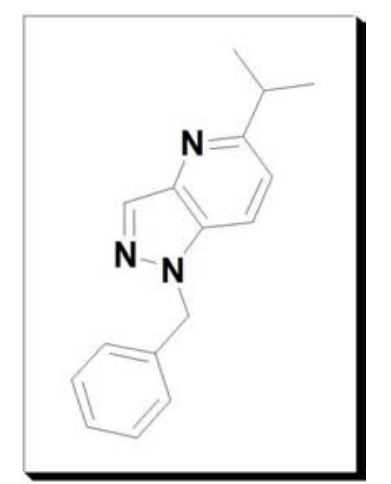

Compound 3: 1H-NMR (400 MHz, CDCl3)

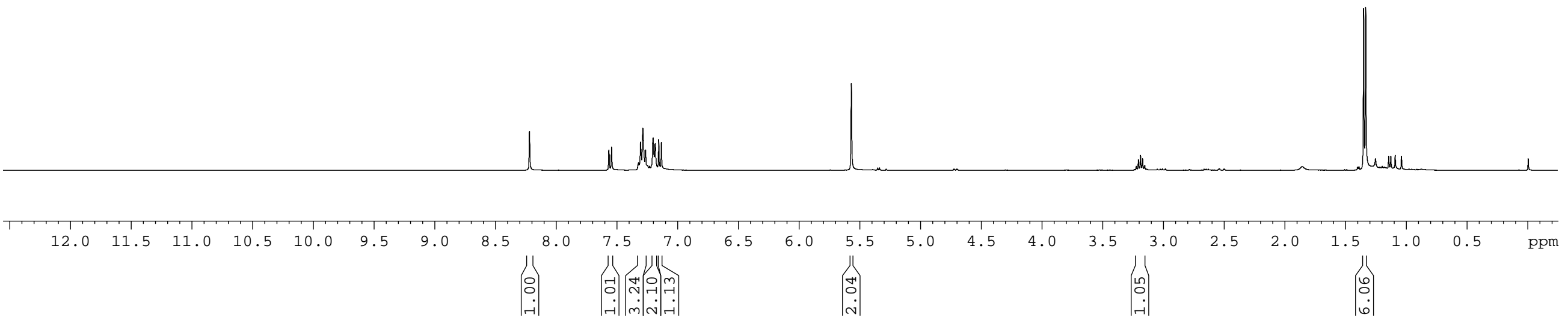




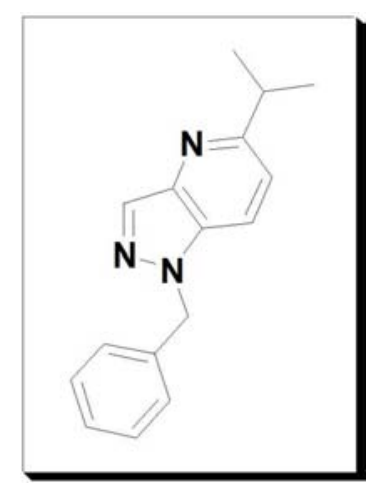

Compound 3: 13C-NMR (100 MHz, CDCl3)

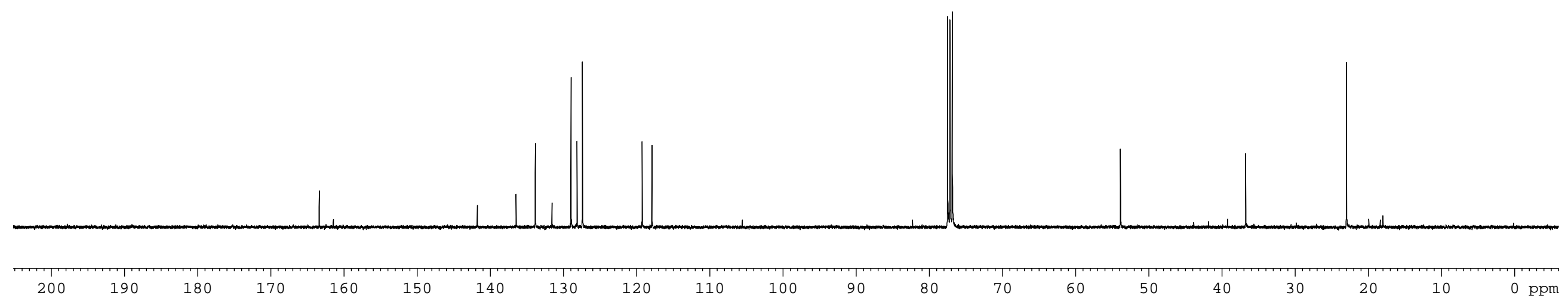




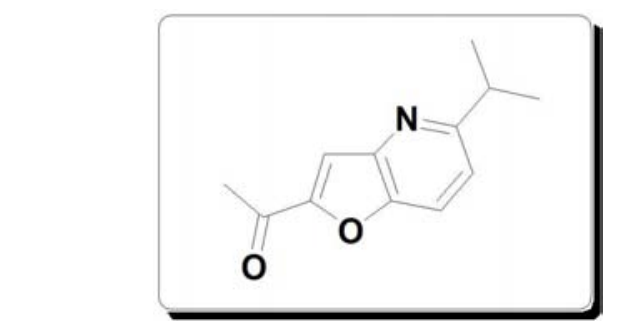

Compound 4a: 1H-NMR (400 MHz, CDCl3)

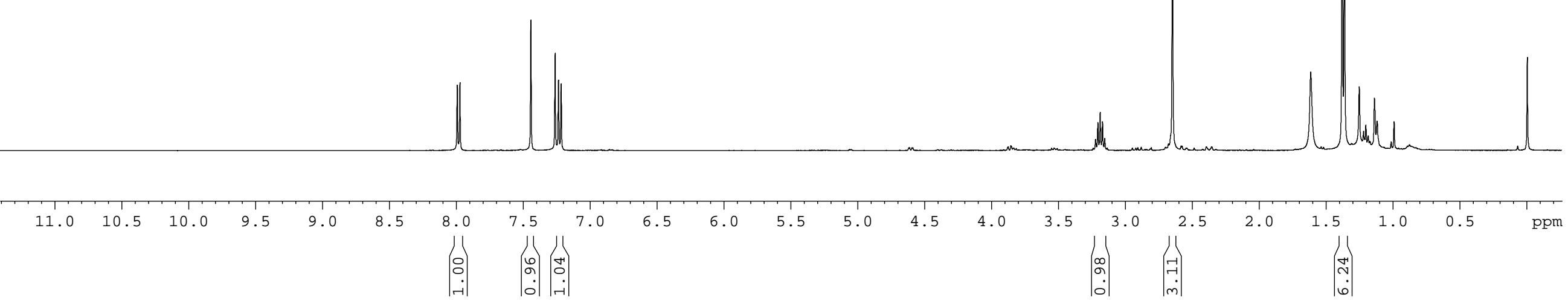




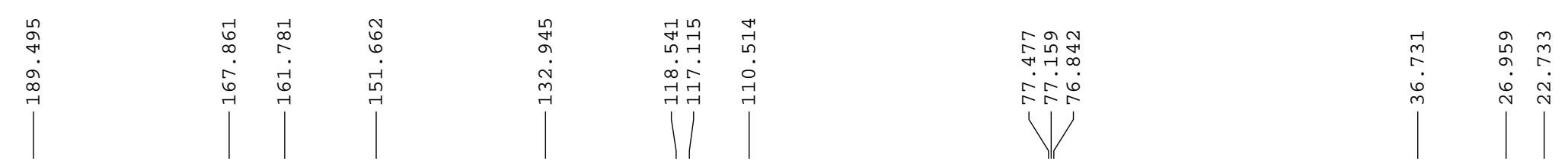

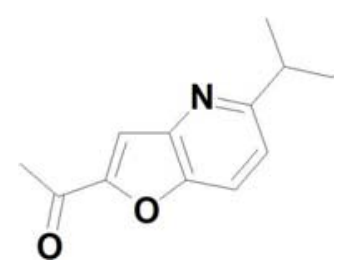

Compound 4a: 13C-NMR (100 MHz, CDCl3)

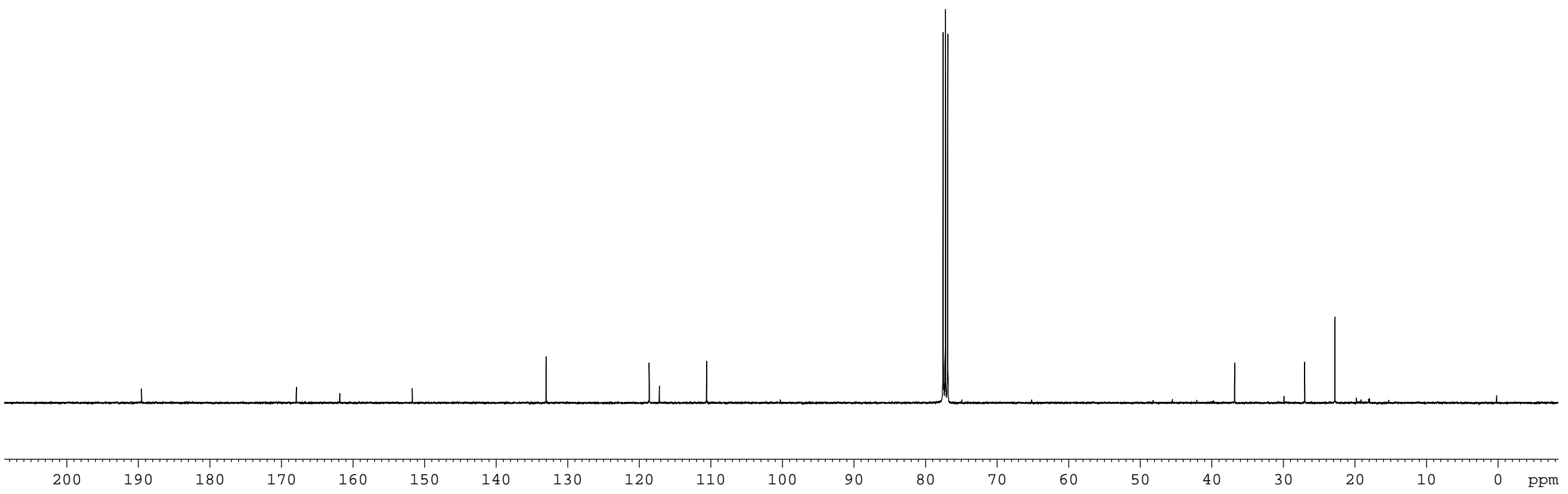




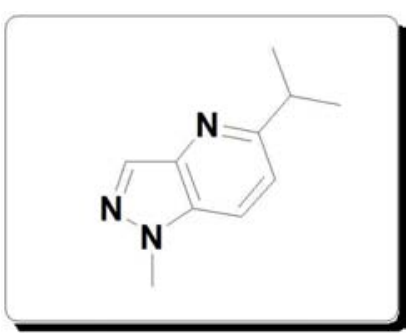

Compound 4b: 1H-NMR (400 MHz, CDCl3)

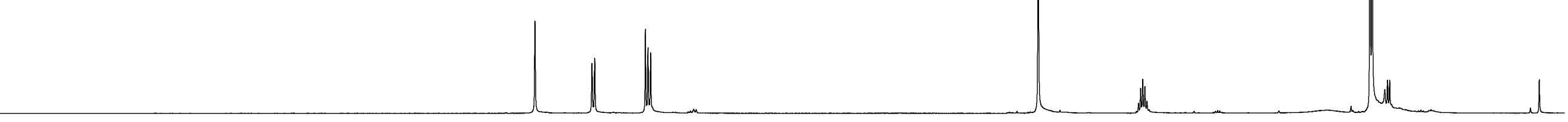

$\begin{array}{llllllll}12.0 & 11.5 & 11.0 & 10.5 & 10.0 & 9.5 & 9.0 & 8.5\end{array}$

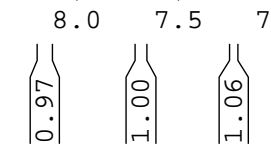
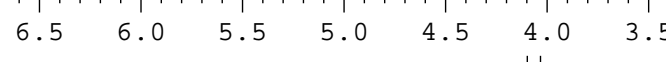

$\left|\begin{array}{ll}4.0 & 3.5 \\ 0 \\ 0 \\ m\end{array}\right| \begin{gathered}2 \\ 0\end{gathered}$

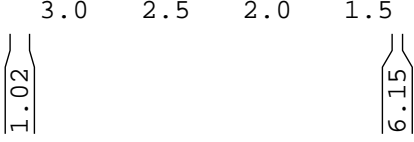




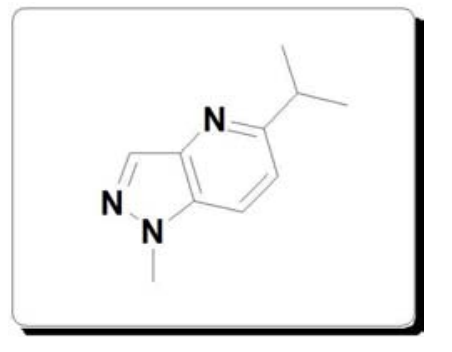

Compound 4b: 13C-NMR (100 MHz, CDCl3)

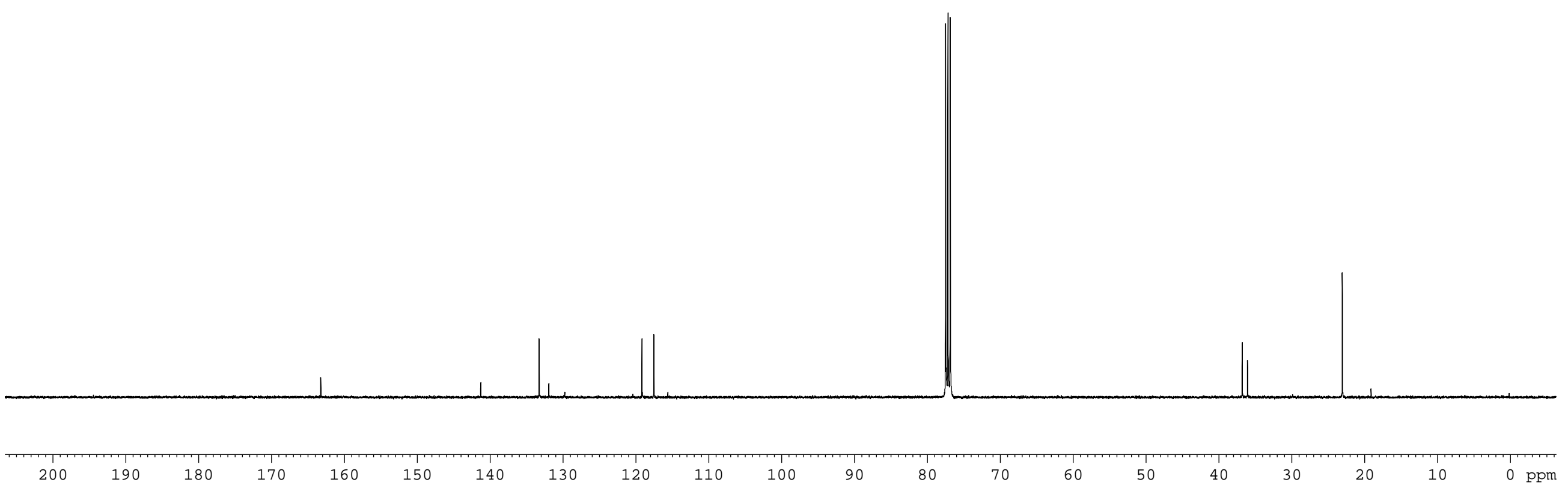




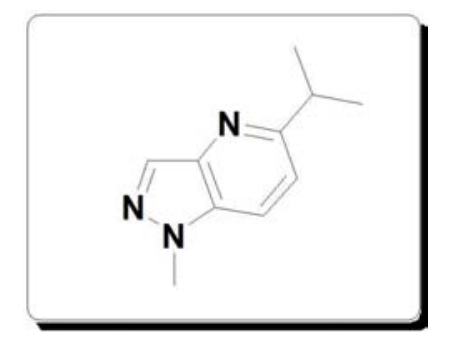

Compound 4b: NOE (400 MHz, CDCl3)

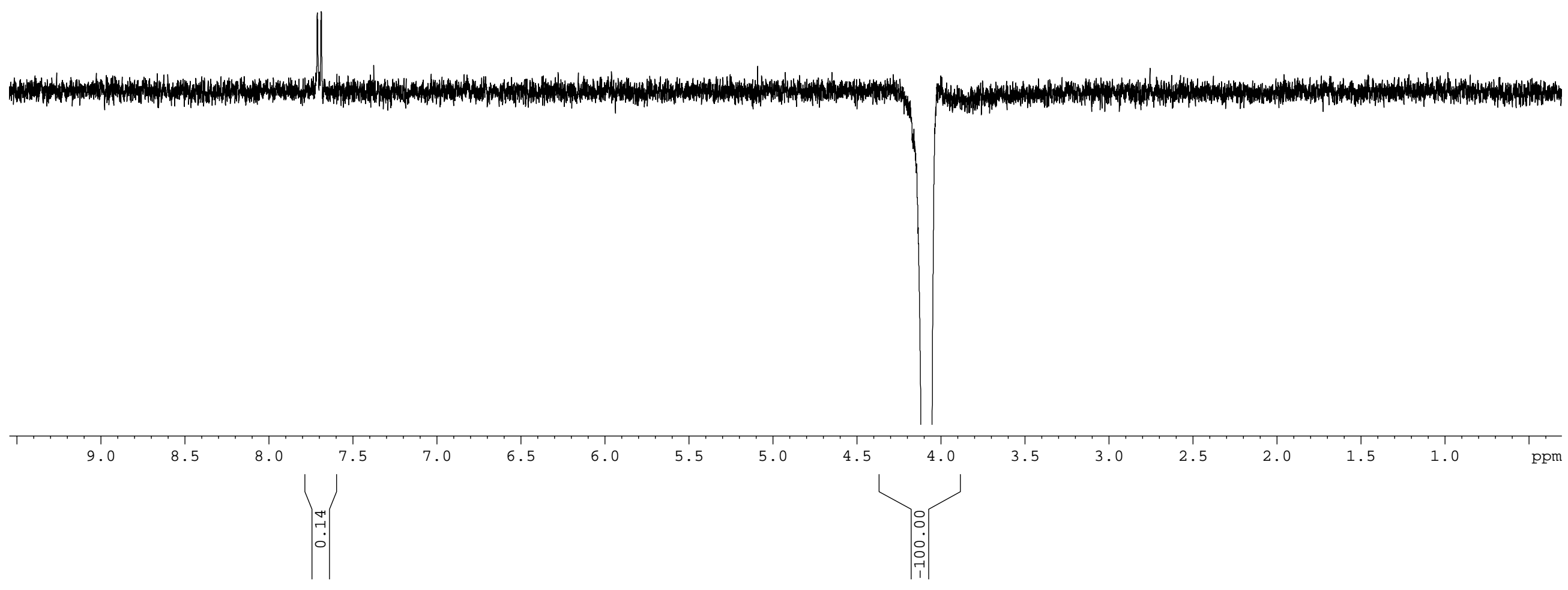




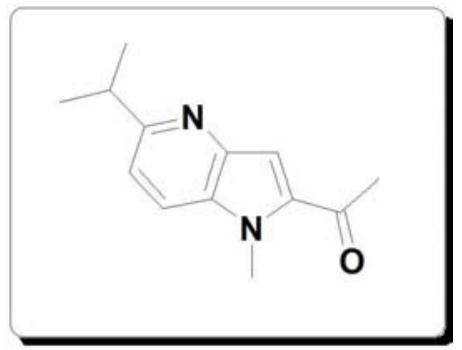

Compound 4c: 1H-NMR (400 MHz, CDCl3)

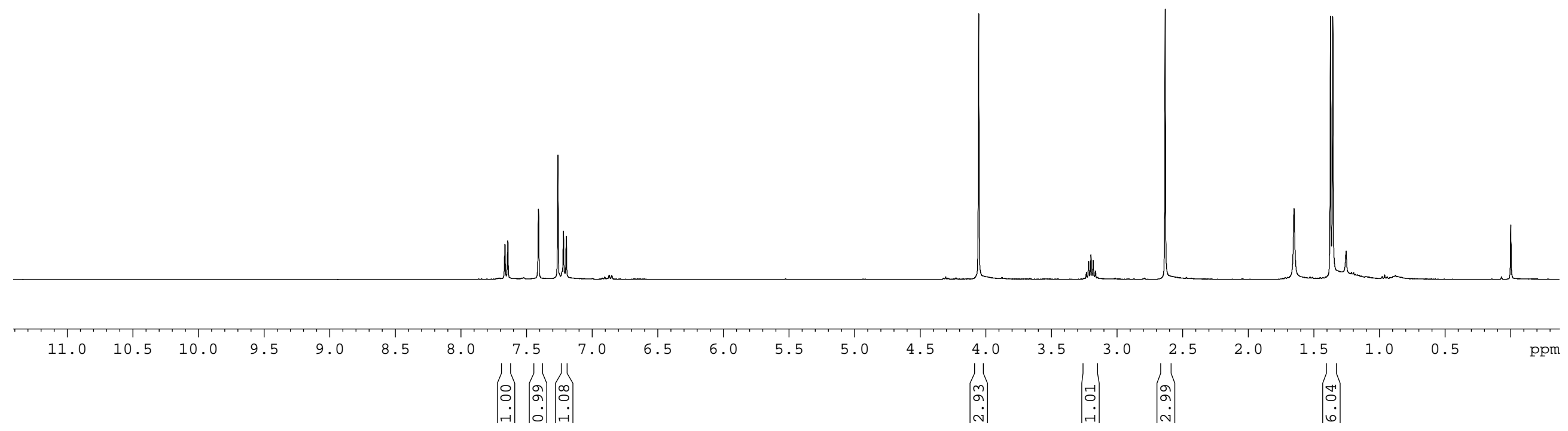




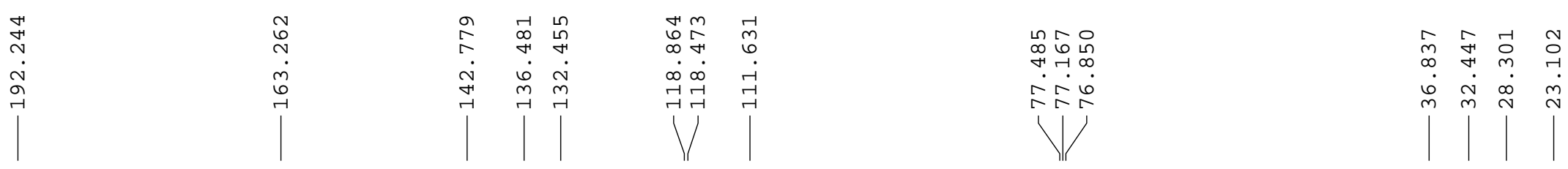

Compound 4c: 13C-NMR (100 MHz, CDCl3)
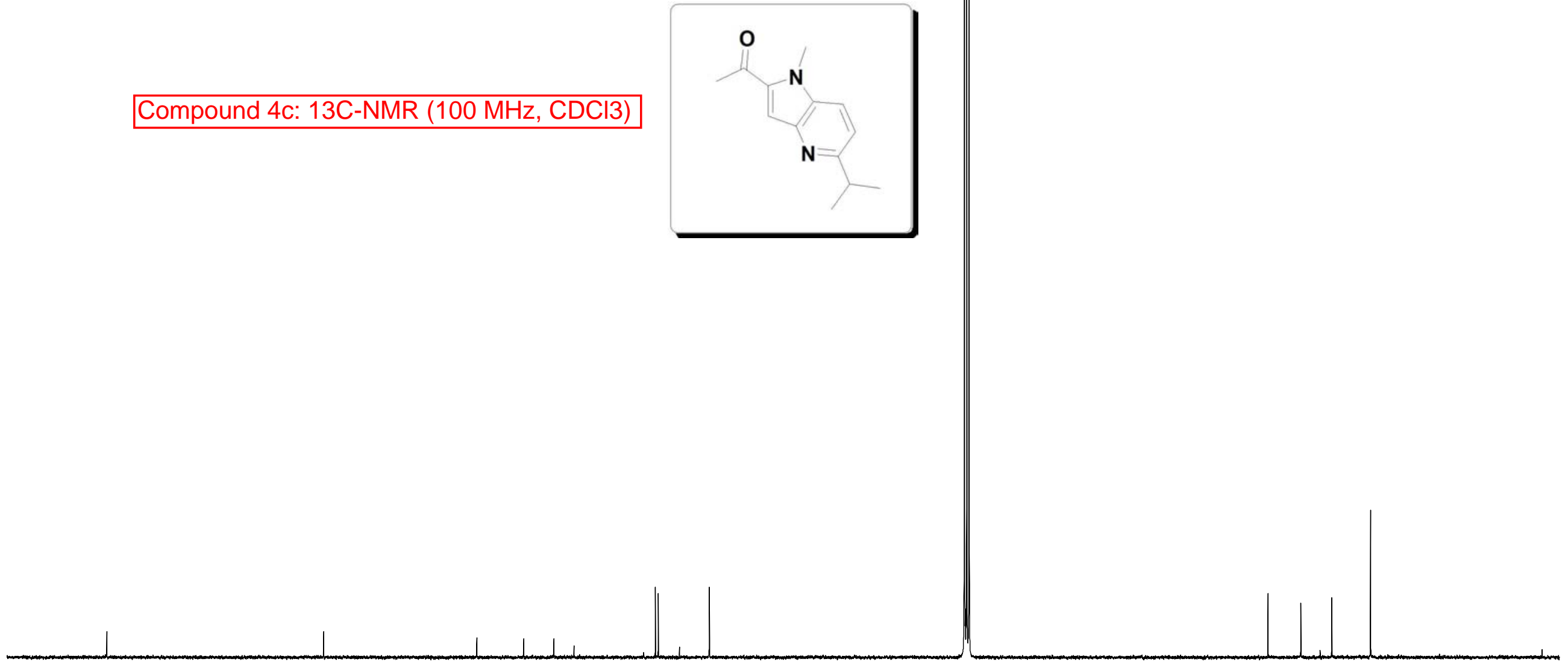


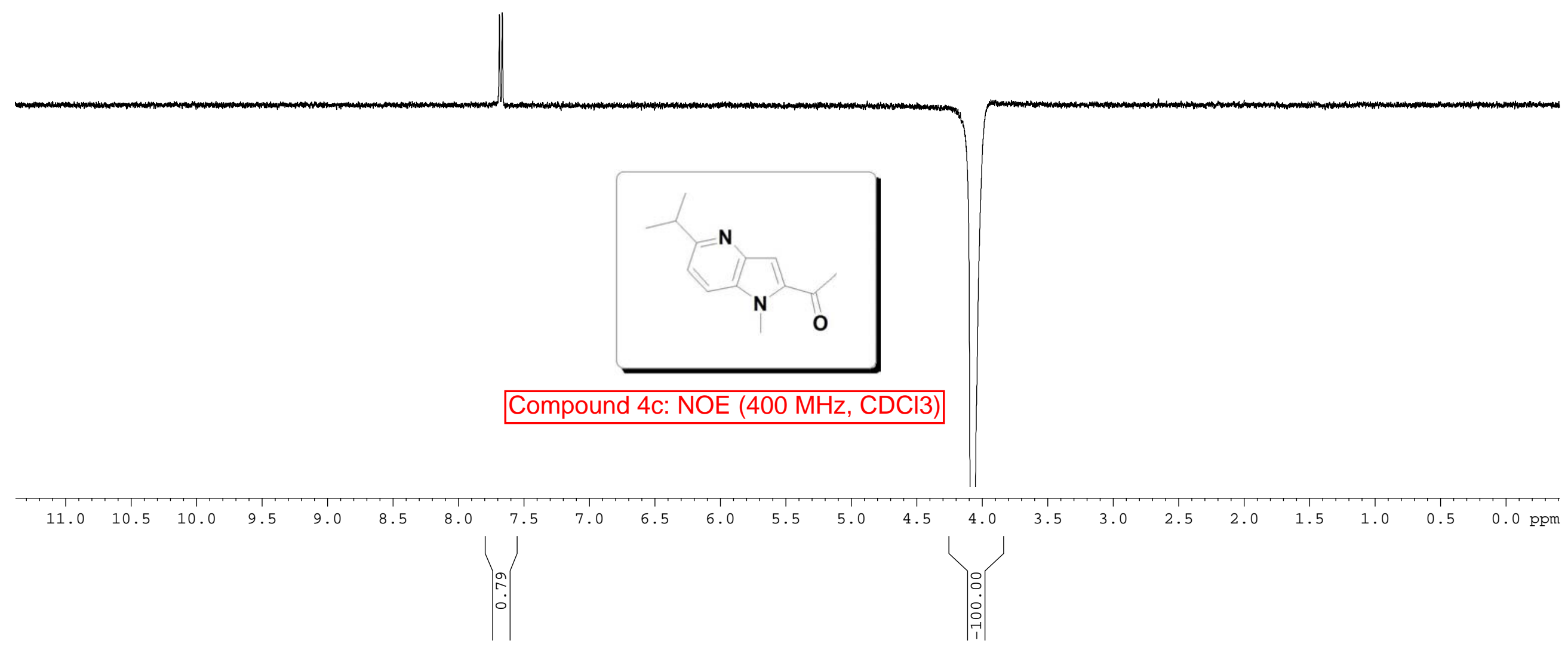




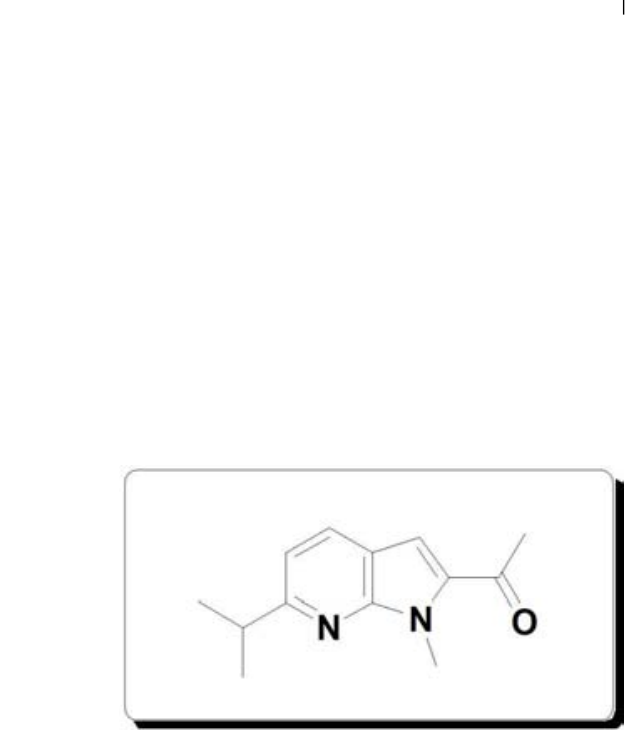

Compound 4d: 1H-NMR (400 MHz, CDCl3)

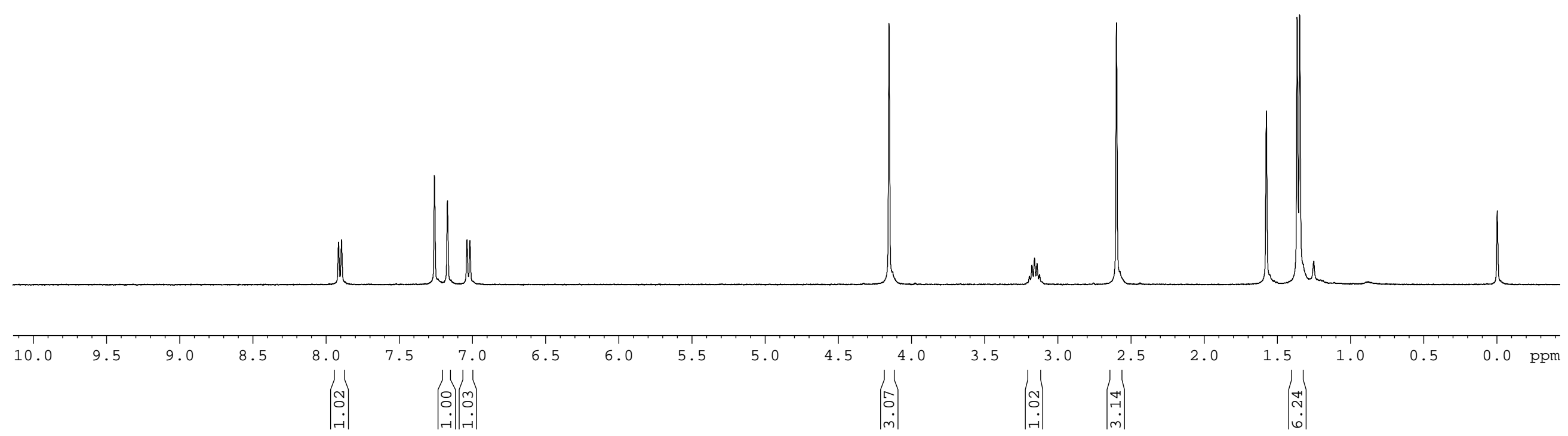




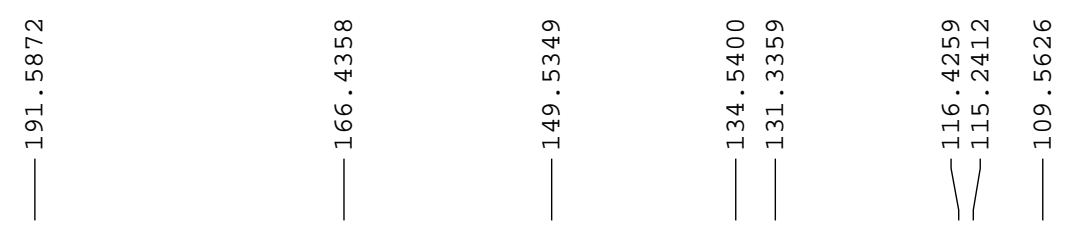

Current Data Parameters

NAME $\quad$ ly8-p74-C-2-08262015

EXPNO

20

PROCNO

1

F2 - Acquisition Parameters

Date_ 20150827

Time $\quad 5.57$

INSTRUM spect

PROBHD $5 \mathrm{~mm}$ PABBO BB-

PULPROG ZgPg30

TD

SOLVENT CDCl3

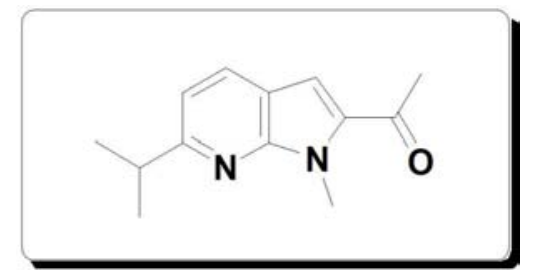

Compound 4d: 13C-NMR (100 MHz, CDCl3)

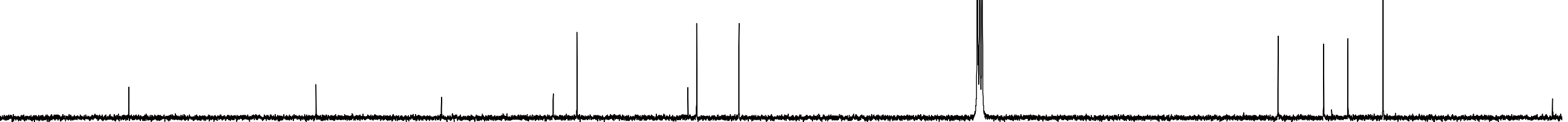




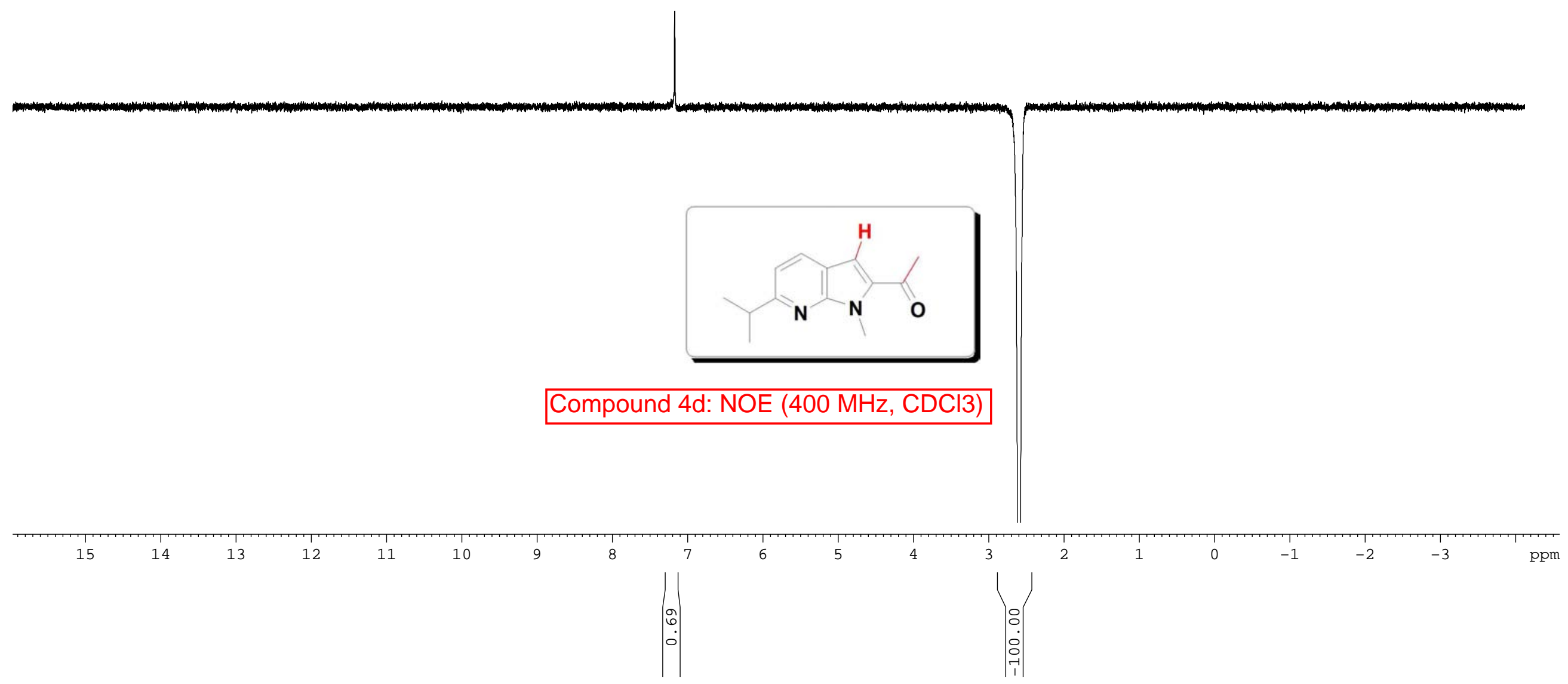




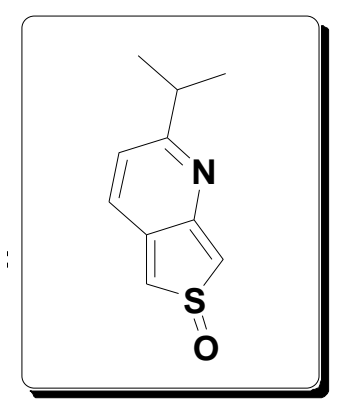

Compound 4e: 1H-NMR (400 MHz, CDCl3)

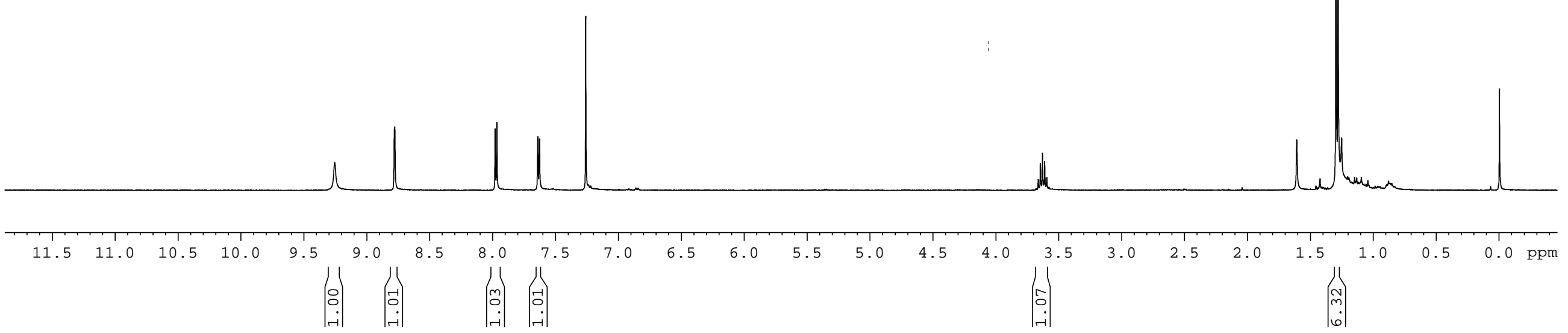




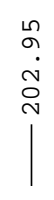
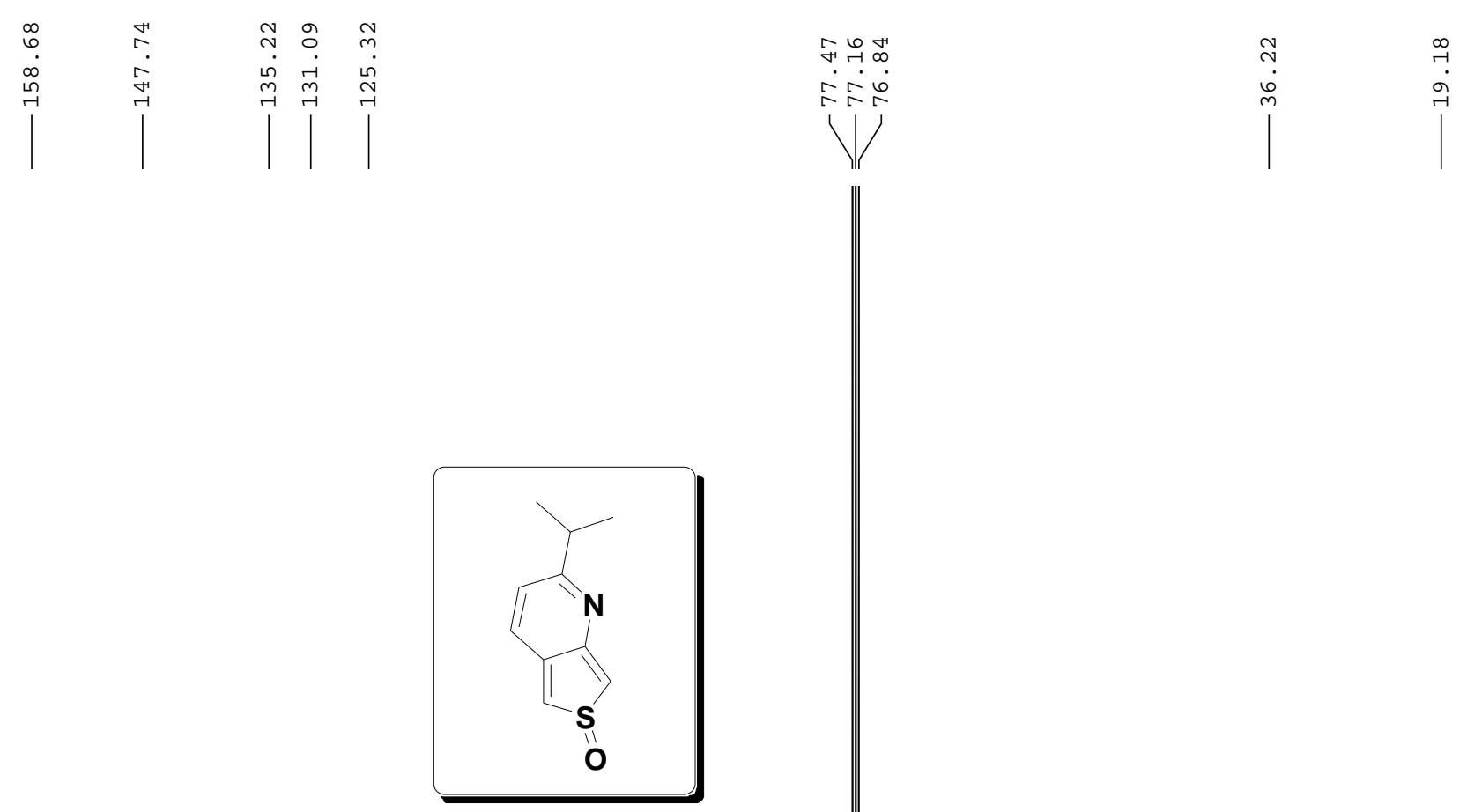

Compound 4e: 13C-NMR (100 MHz, CDCl3)

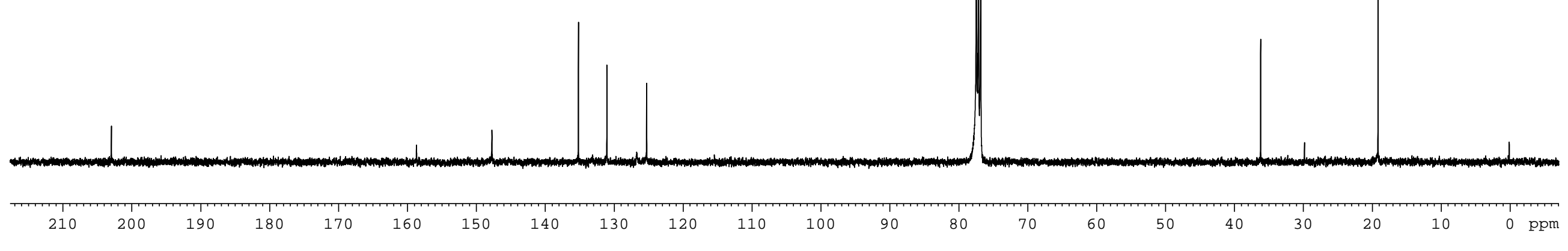




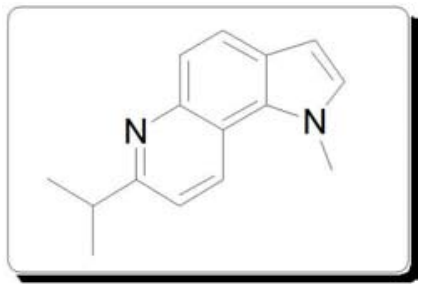

Compound 4f: 1H-NMR (400 MHz, CDCl3)

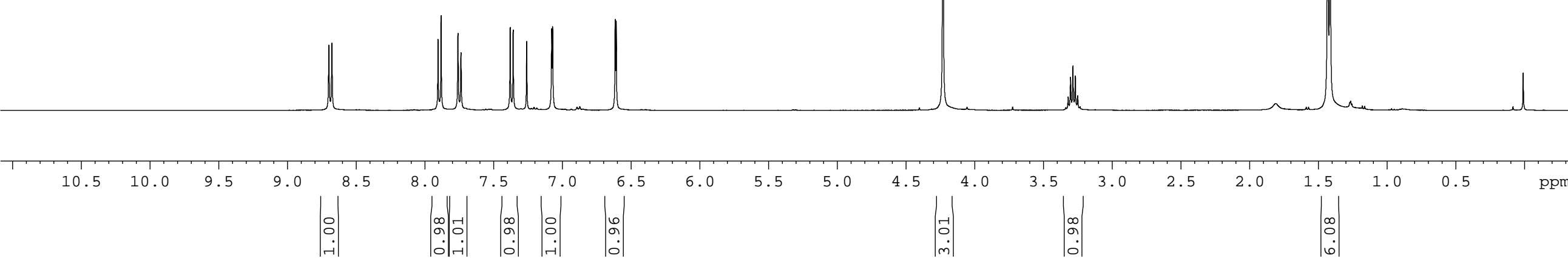




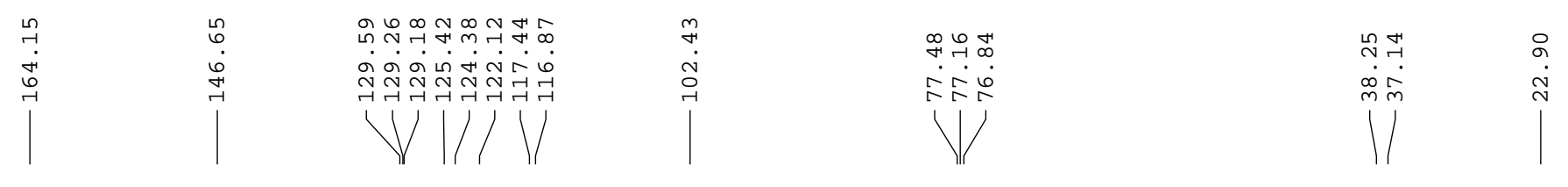

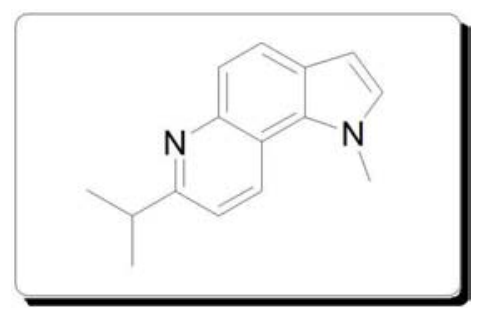

Compound 4f: 13C-NMR (100 MHz, CDCl3)

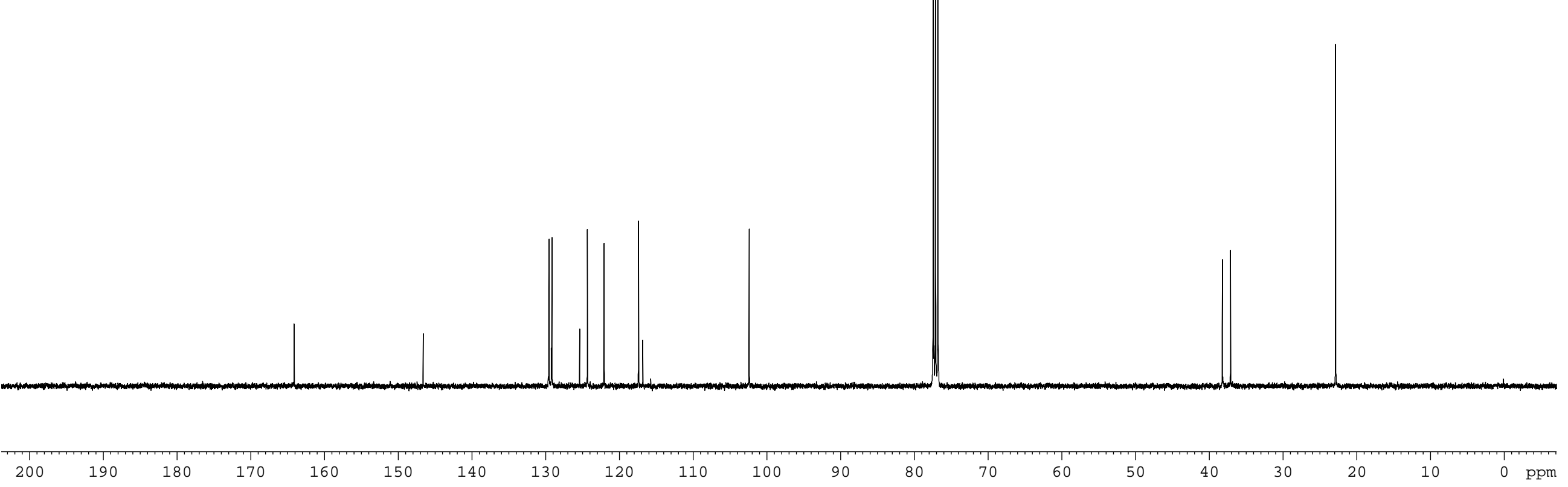




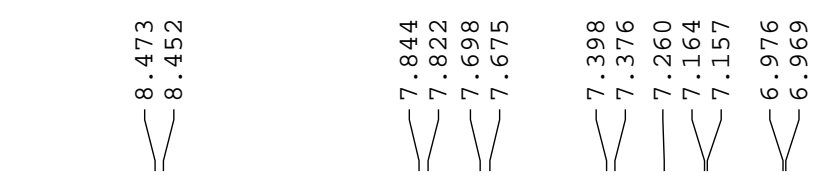

Compound 4g: 1H-NMR (400 MHz, CDCl3)
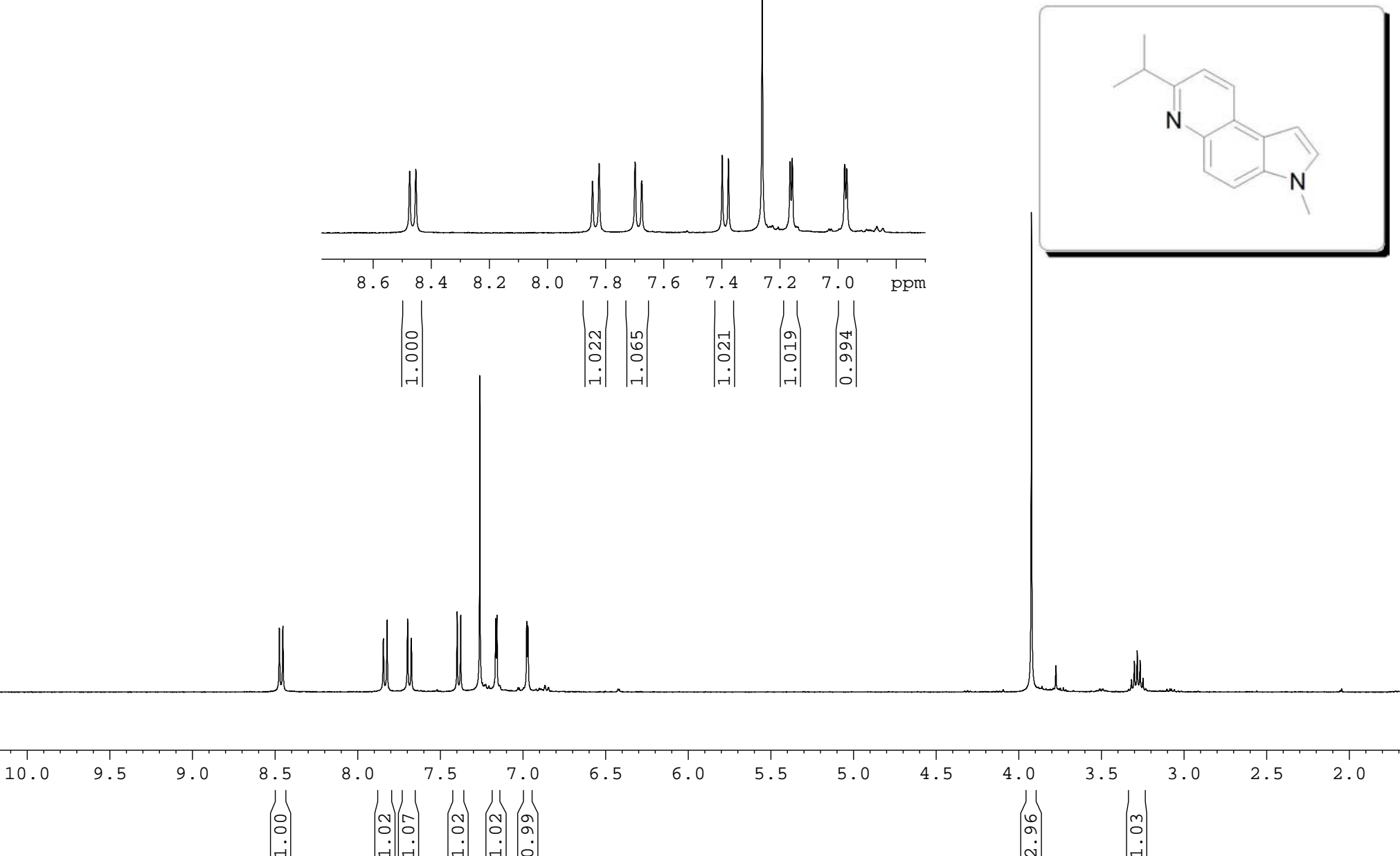

$\left(\begin{array}{ll}0 & 3.5 \\ 0 & \\ 0 & \end{array}\right.$ 
$\vec{n}$
$\dot{0}$
$\stackrel{0}{0}$

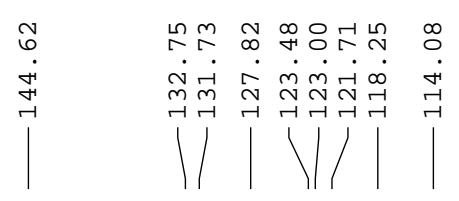

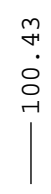

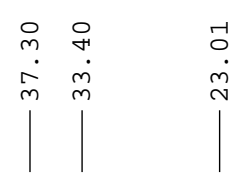

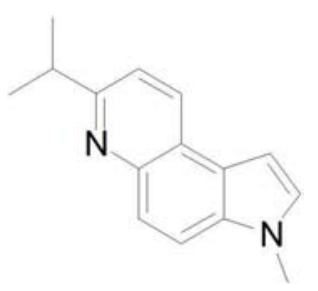

Compound 4g: 13C-NMR (100 MHz, CDCl3)

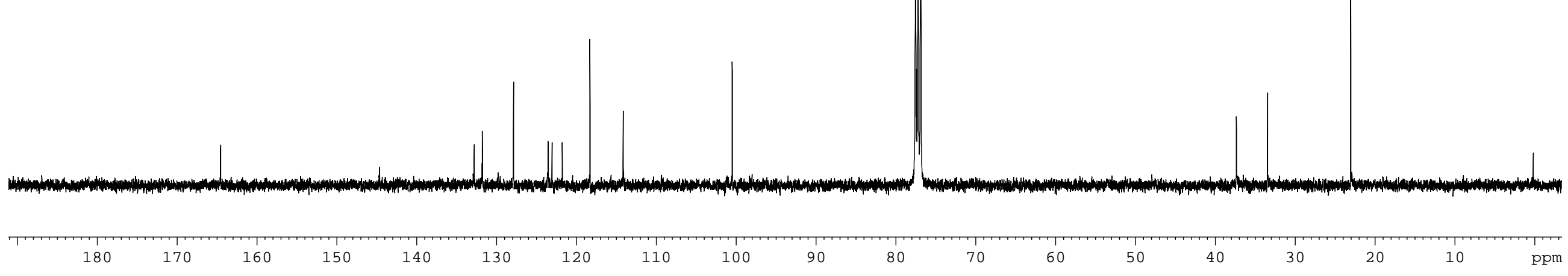




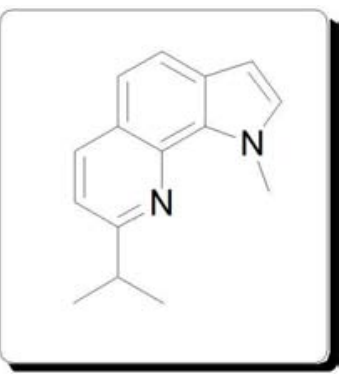

Compound 4h: 1H-NMR (400 MHz, CDCl3) 


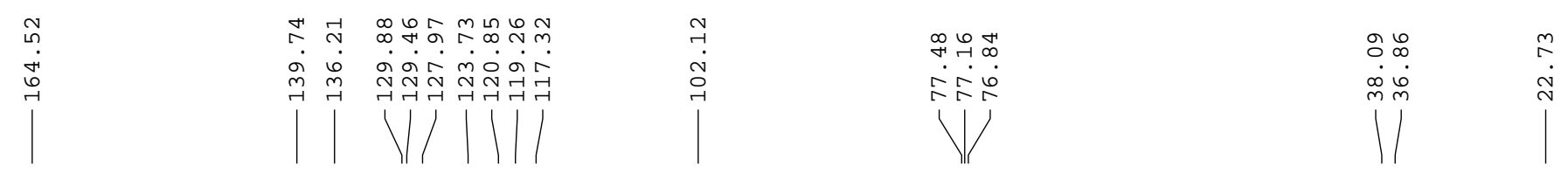

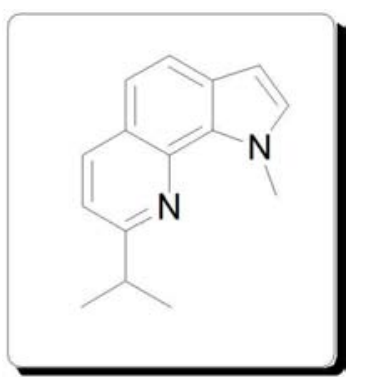

Compound 4h: 13C-NMR (100 MHz, CDCl3)

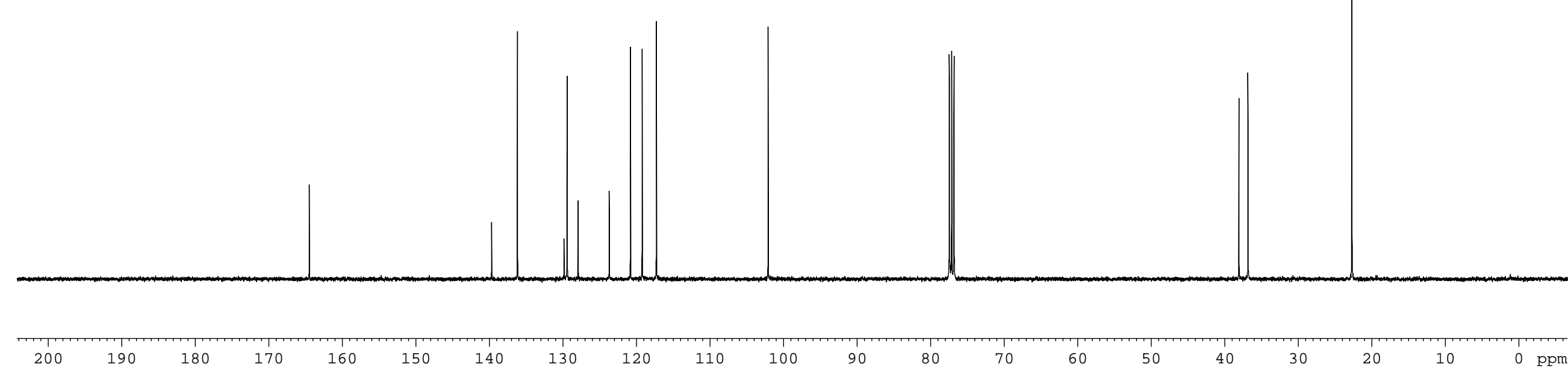



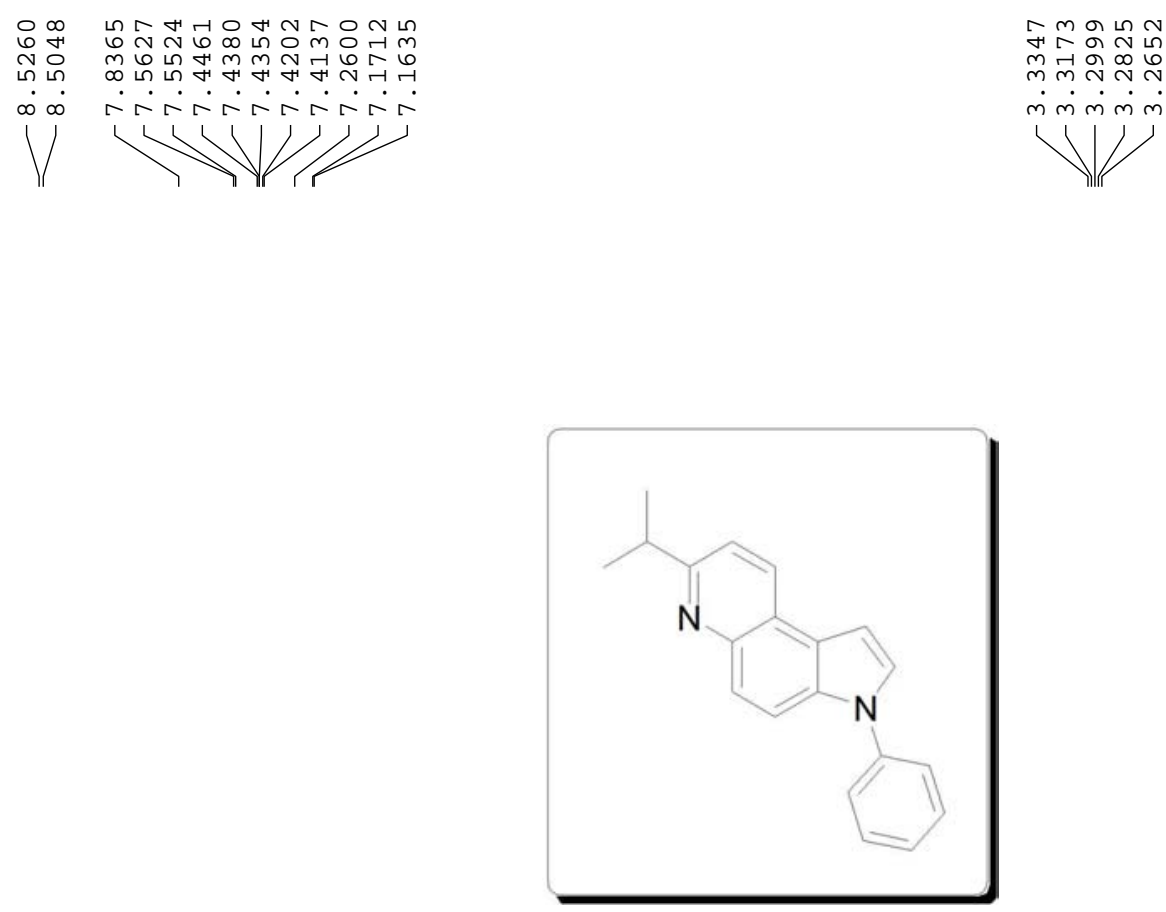

Compound 4i: 1H-NMR (400 MHz, CDCl3)

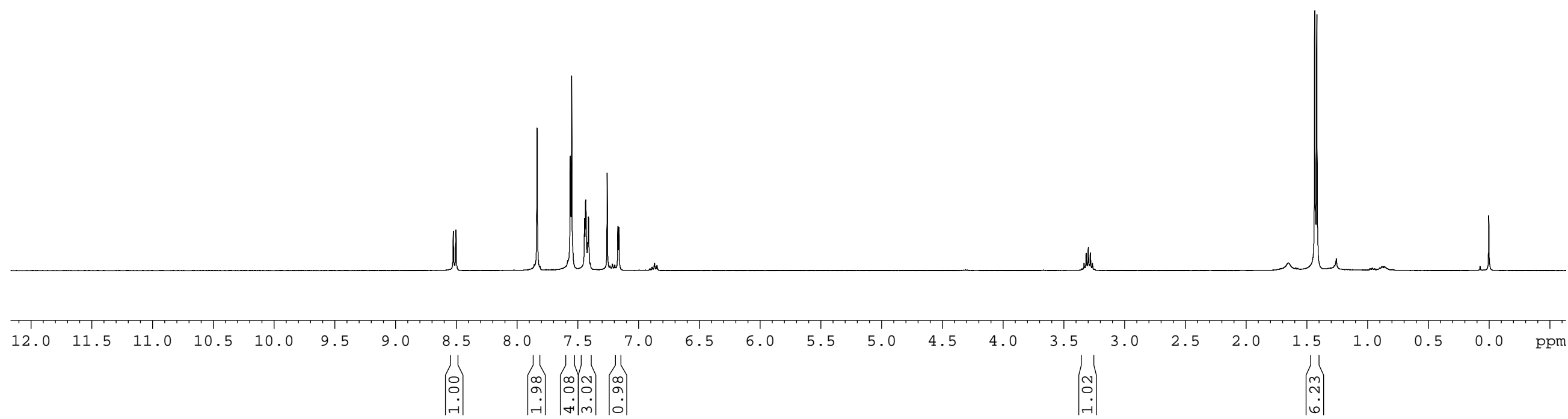




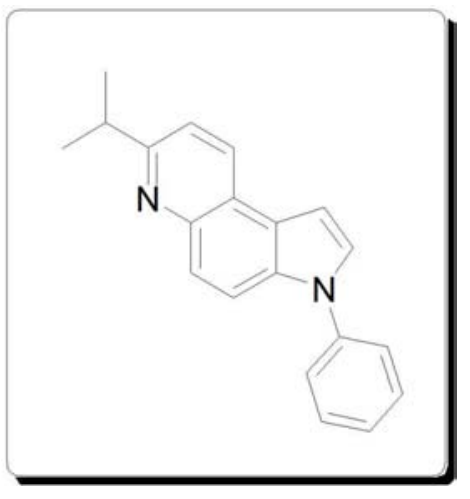

Compound 4i: 13C-NMR (100 MHz, CDCl3)

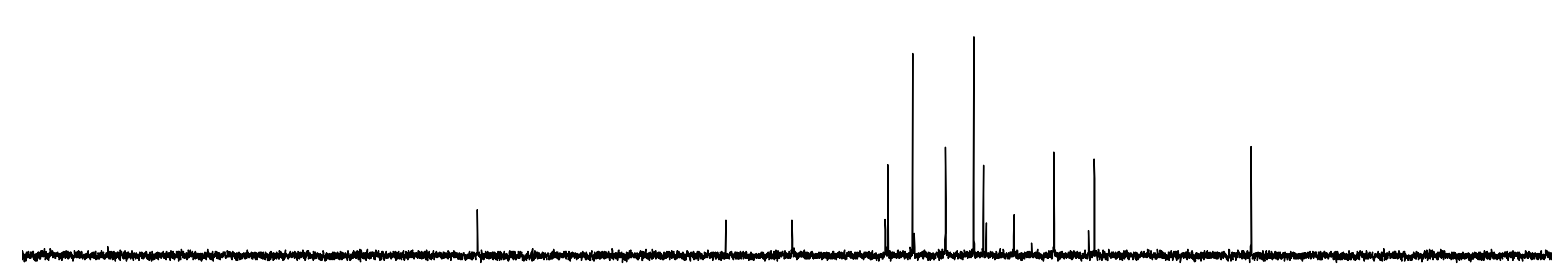




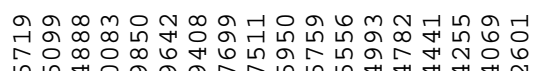

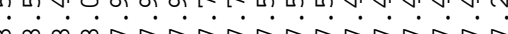

11

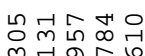

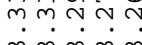

$\|^{m \infty n}$

V일

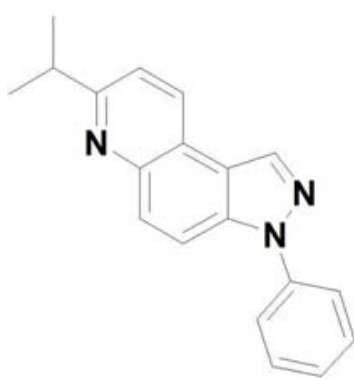

Compound 4j: 1H-NMR (400 MHz, CDCl3)

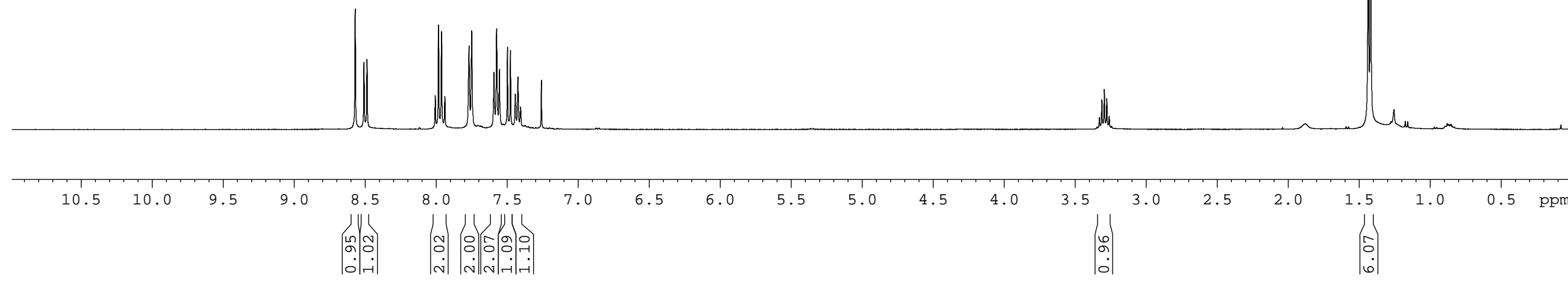




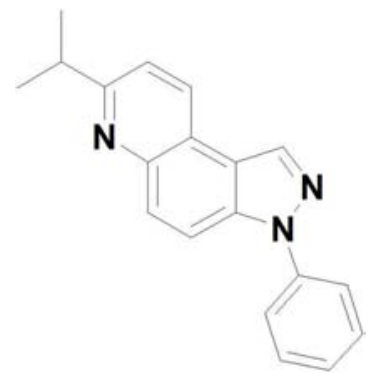

Compound 4j: 13C-NMR (100 MHz, CDCl3)

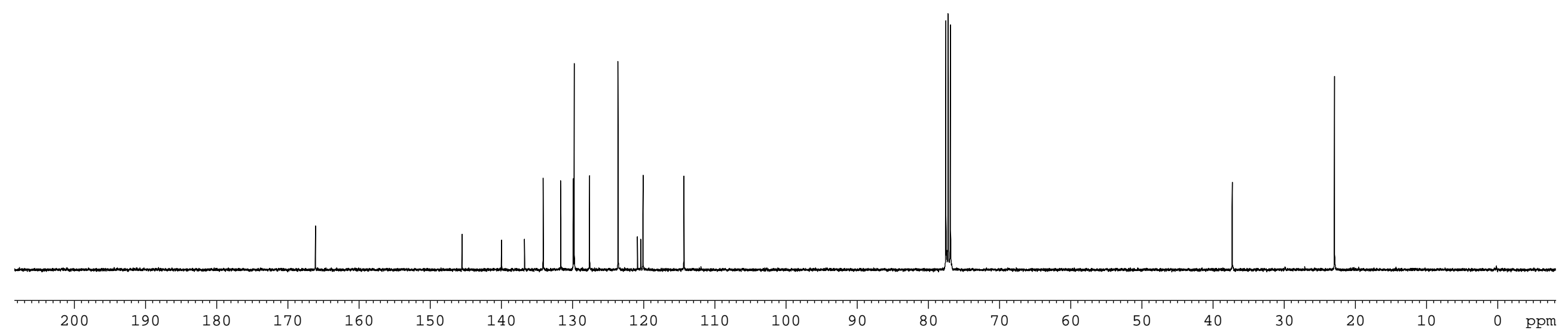




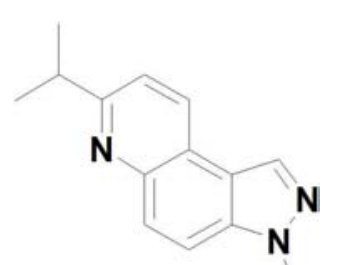

\section{Compound 4k: 1H-NMR (400 MHz, CDCl3)}

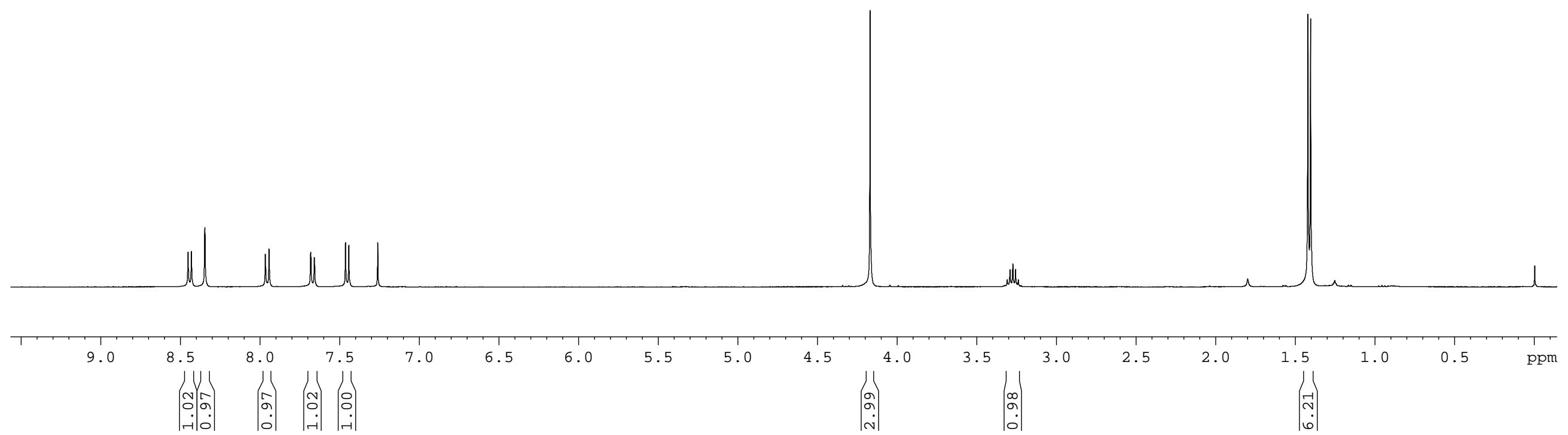




$$
\mathrm{N}^{\mathrm{N}}
$$

Compound 4k: 13C-NMR (100 MHz, CDCl3) 

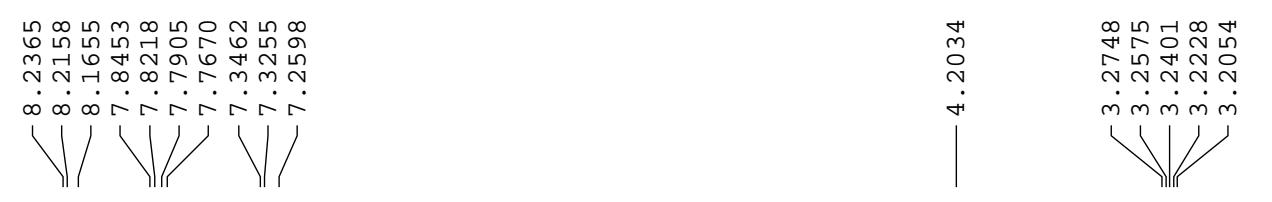

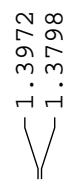

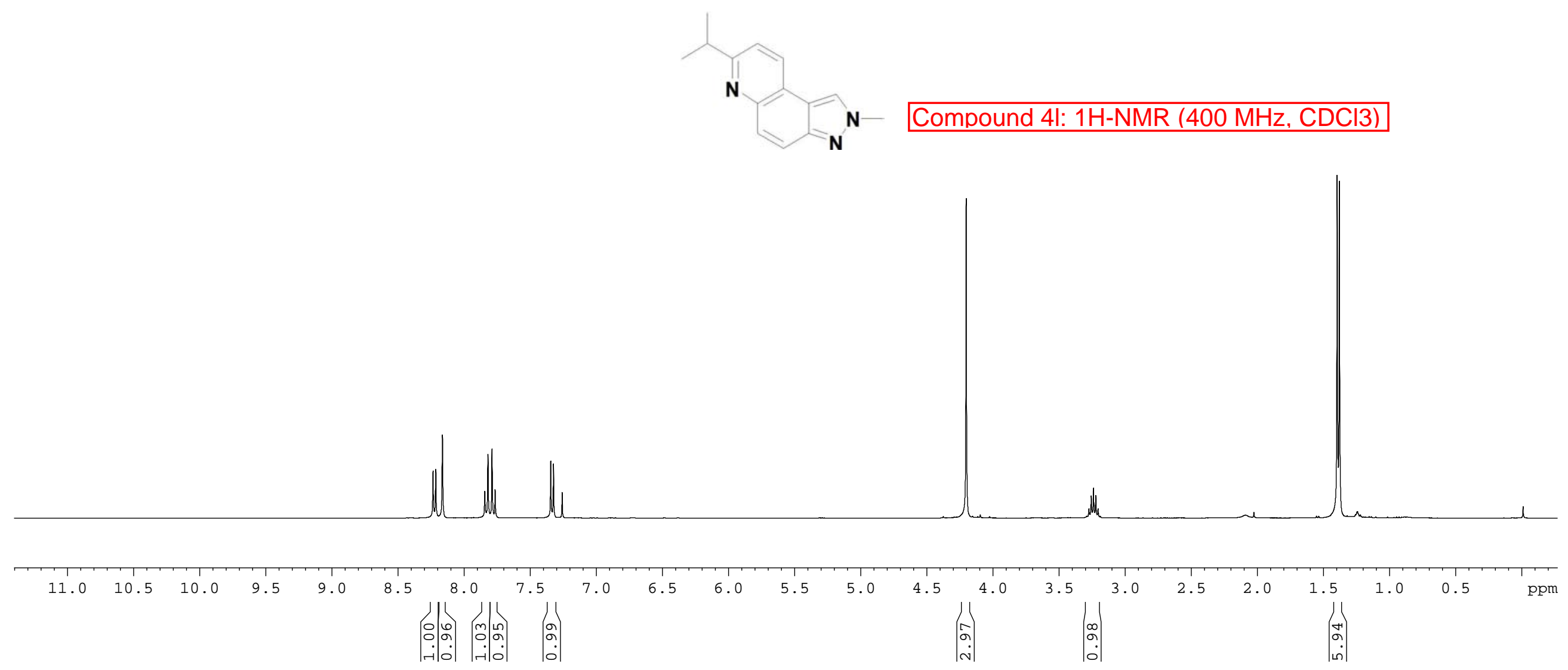



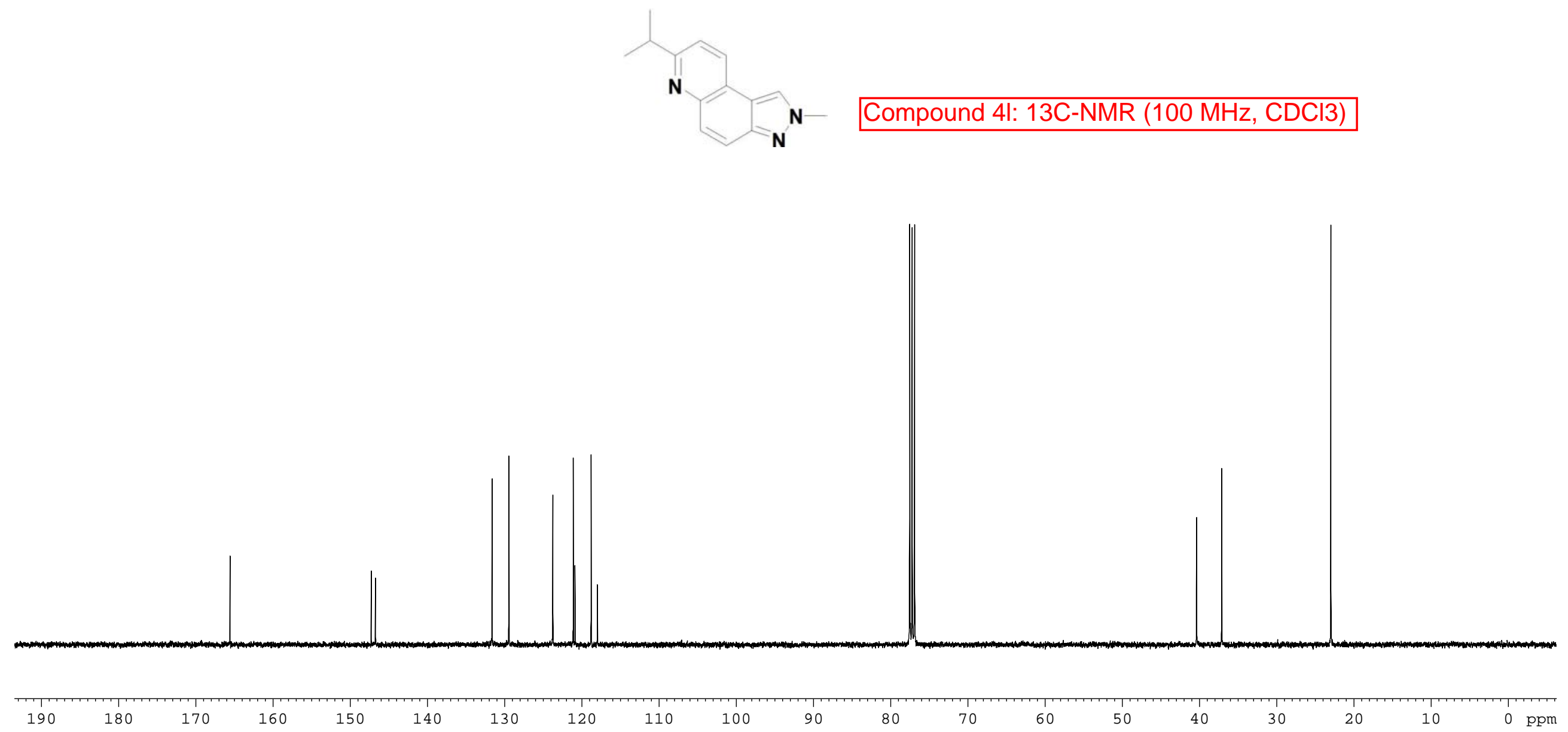



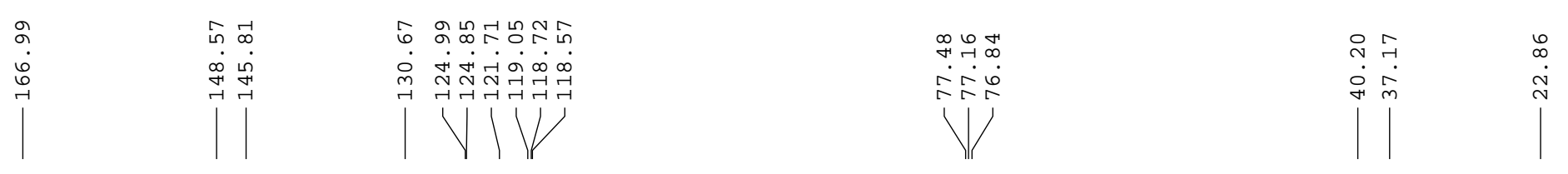

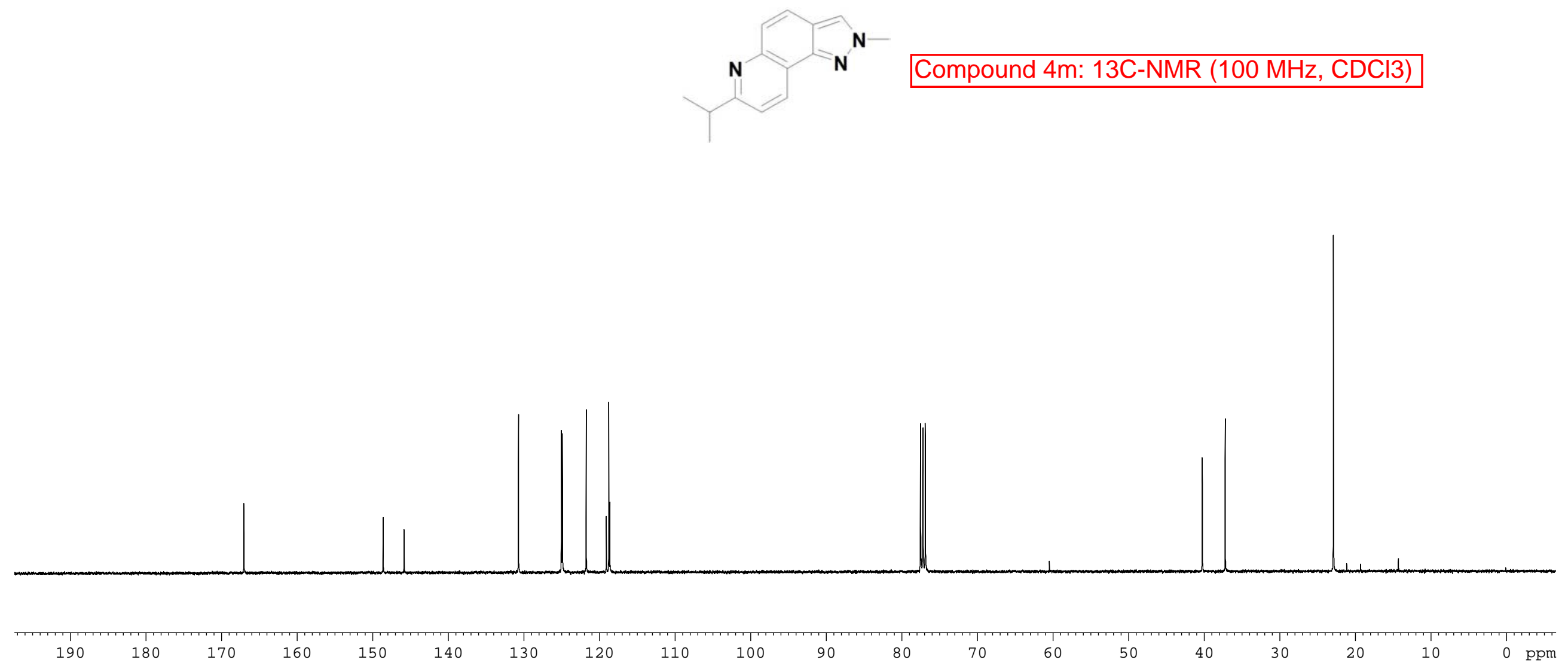




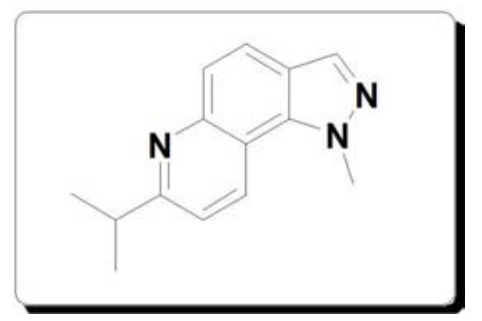

Compound 4n: 1H-NMR (400 MHz, CDCl3)

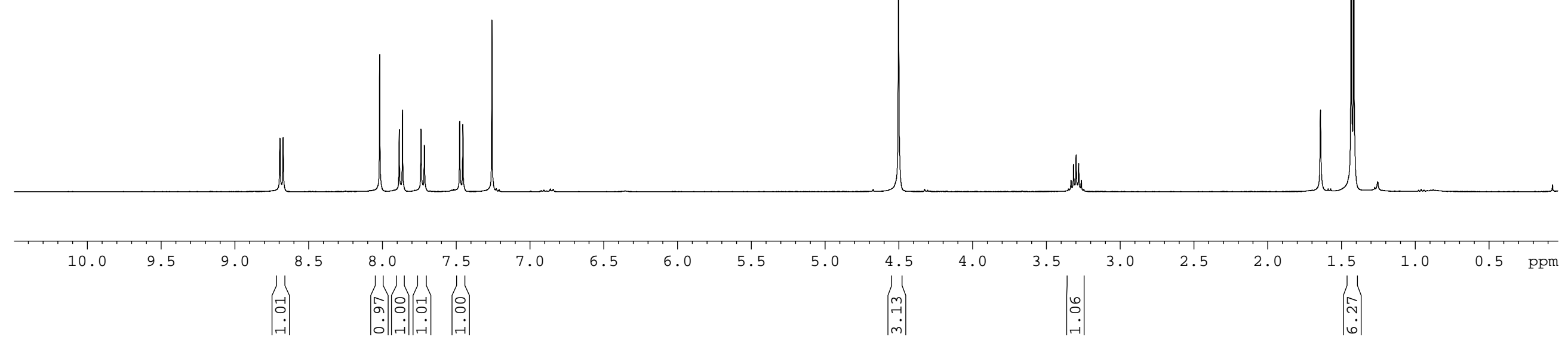




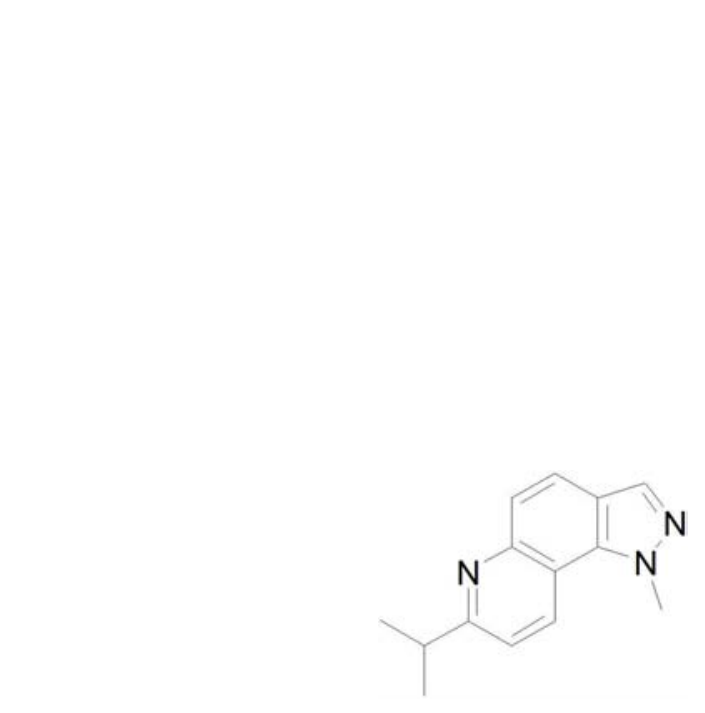

Compound 4n: 13C-NMR (100 MHz, CDCl3)

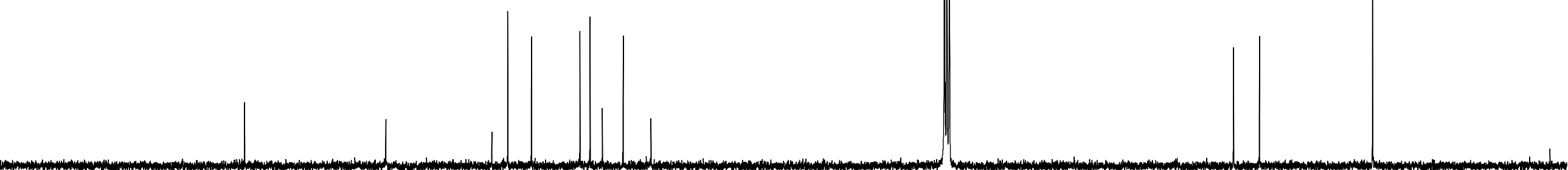



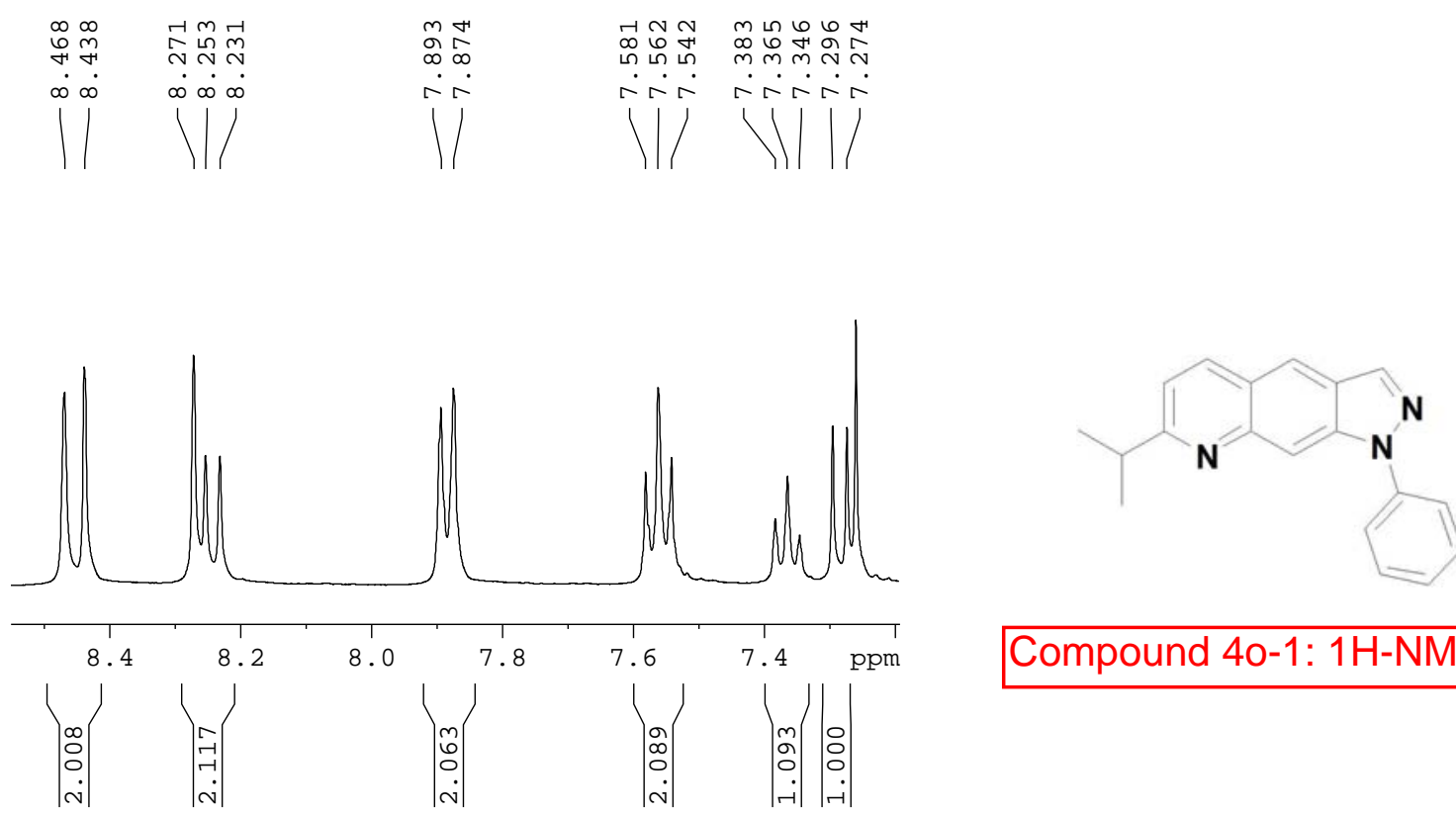

Compound 40-1: 1H-NMR (400 MHz, CDCl3)
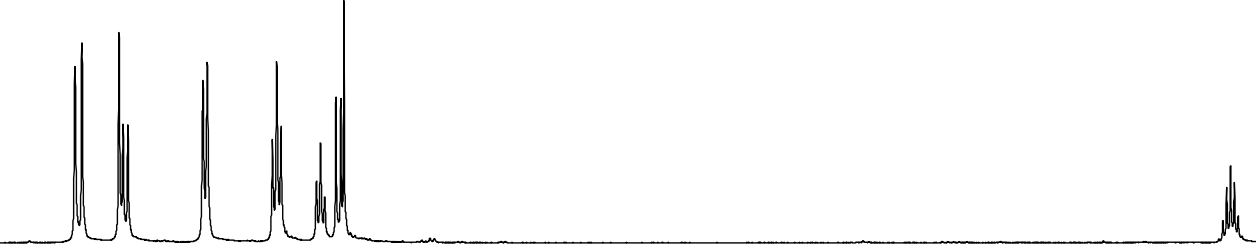

1

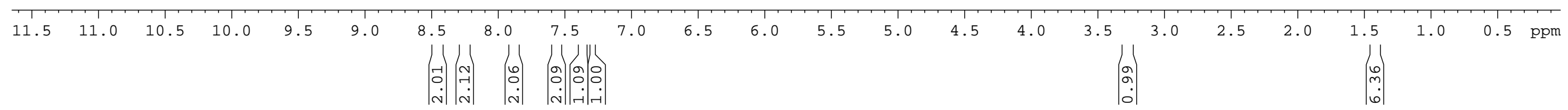


Compound 4o-1: 13C-NMR (100 MHz, CDCl3)

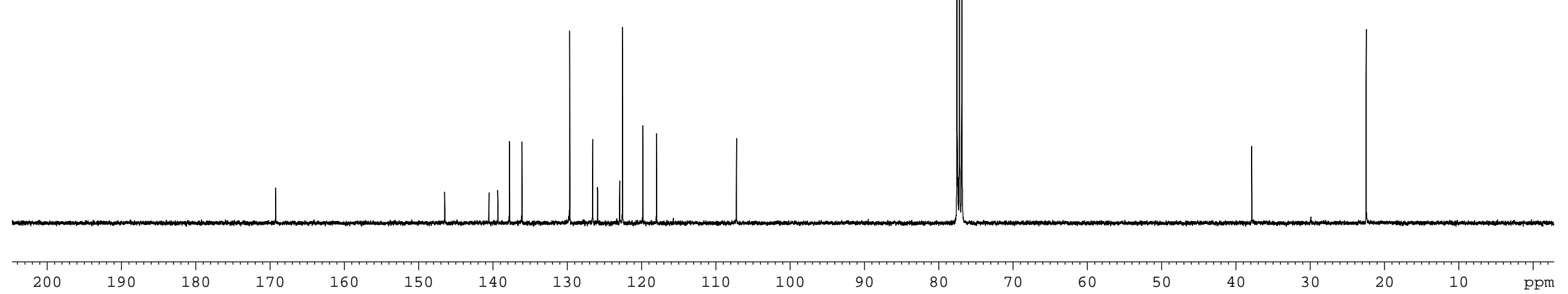




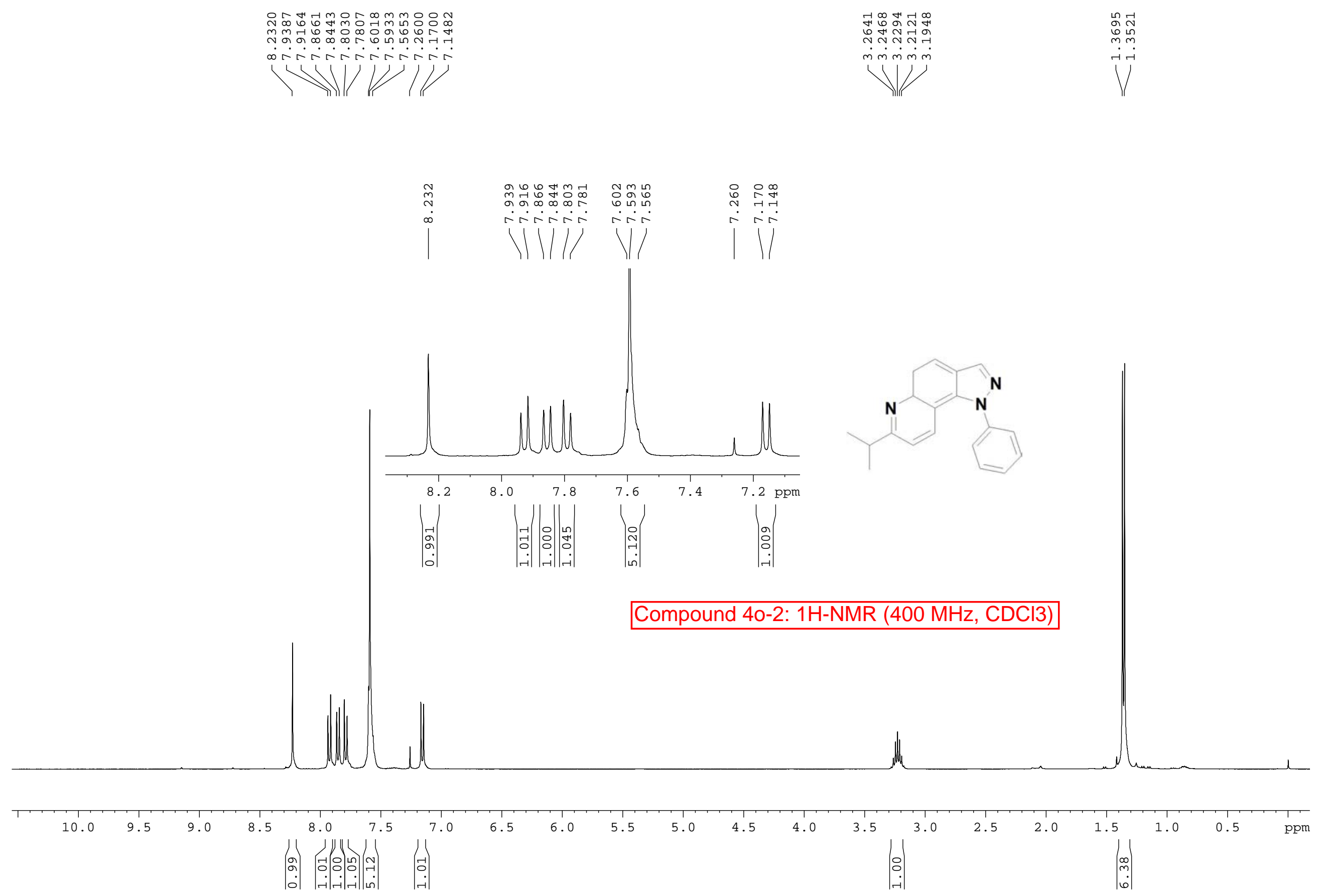




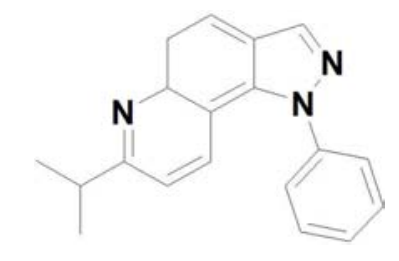

Compound 4o-2: 13C-NMR (100 MHz, CDCl3)

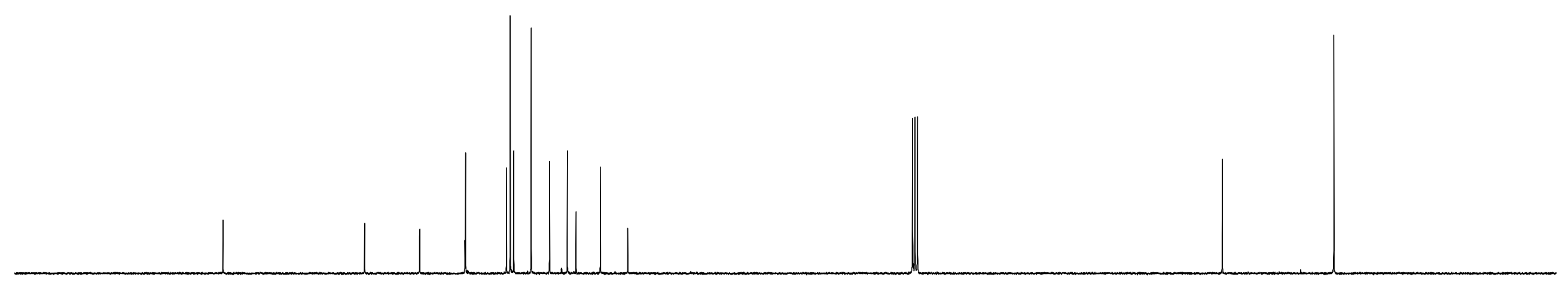


Current Data Parameters

NAME $\quad$ ly8-p102-P-20151015

EXPNO

10

F2 - Acquisition Parameters

Date_ 20151015

INSTRUM

PROBHD $5 \mathrm{~mm}$ PABBO BB-

$\begin{array}{lr}\text { PULPROG } & \text { zg30 } \\ \text { TD } & 65536\end{array}$

SOLVENT

NS

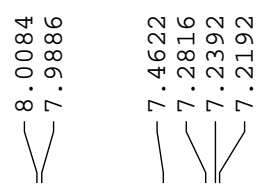

Compound 5a: 1H-NMR (400 MHz, CDCl3)

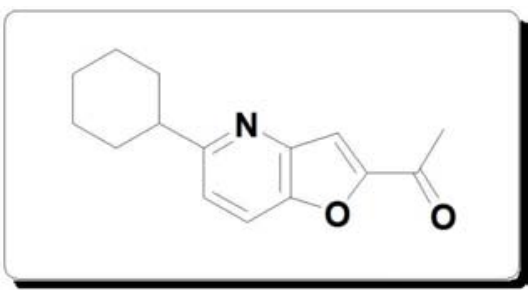

CDC13

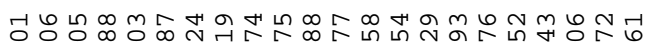

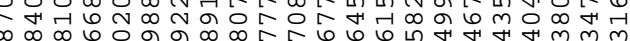

حरें

11

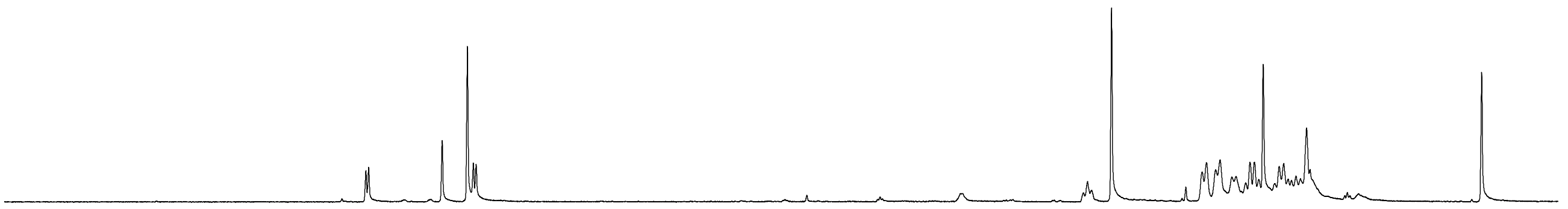

10.0

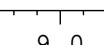

8.5

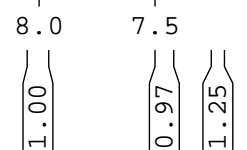

$5.0 \quad 4.5$

4.0

3.5

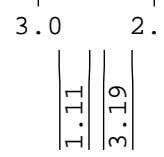

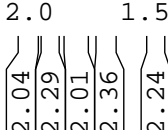

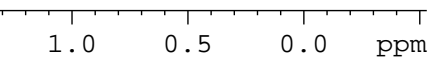



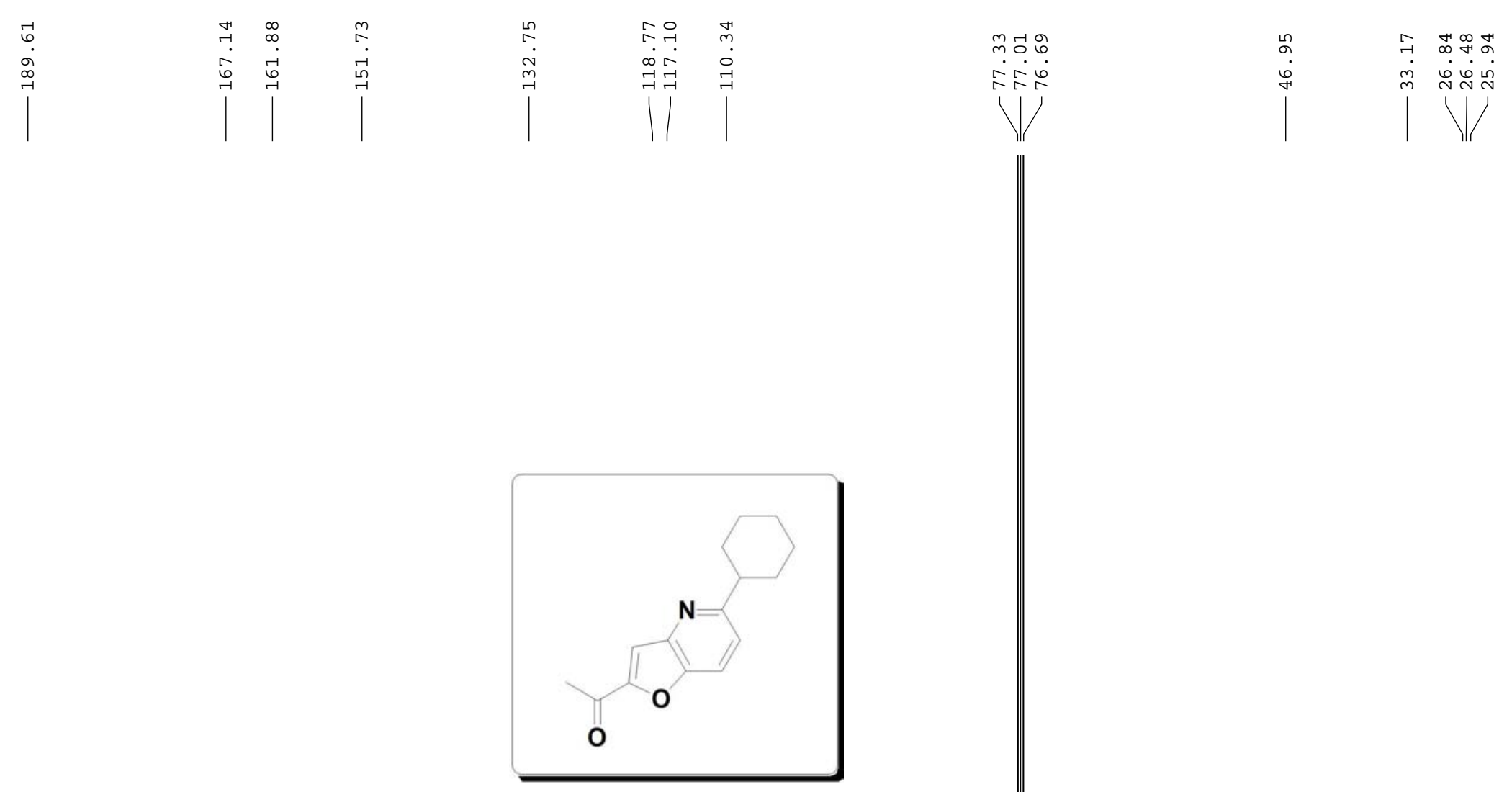

Compound 5a: 13C-NMR (100 MHz, CDCl3) 


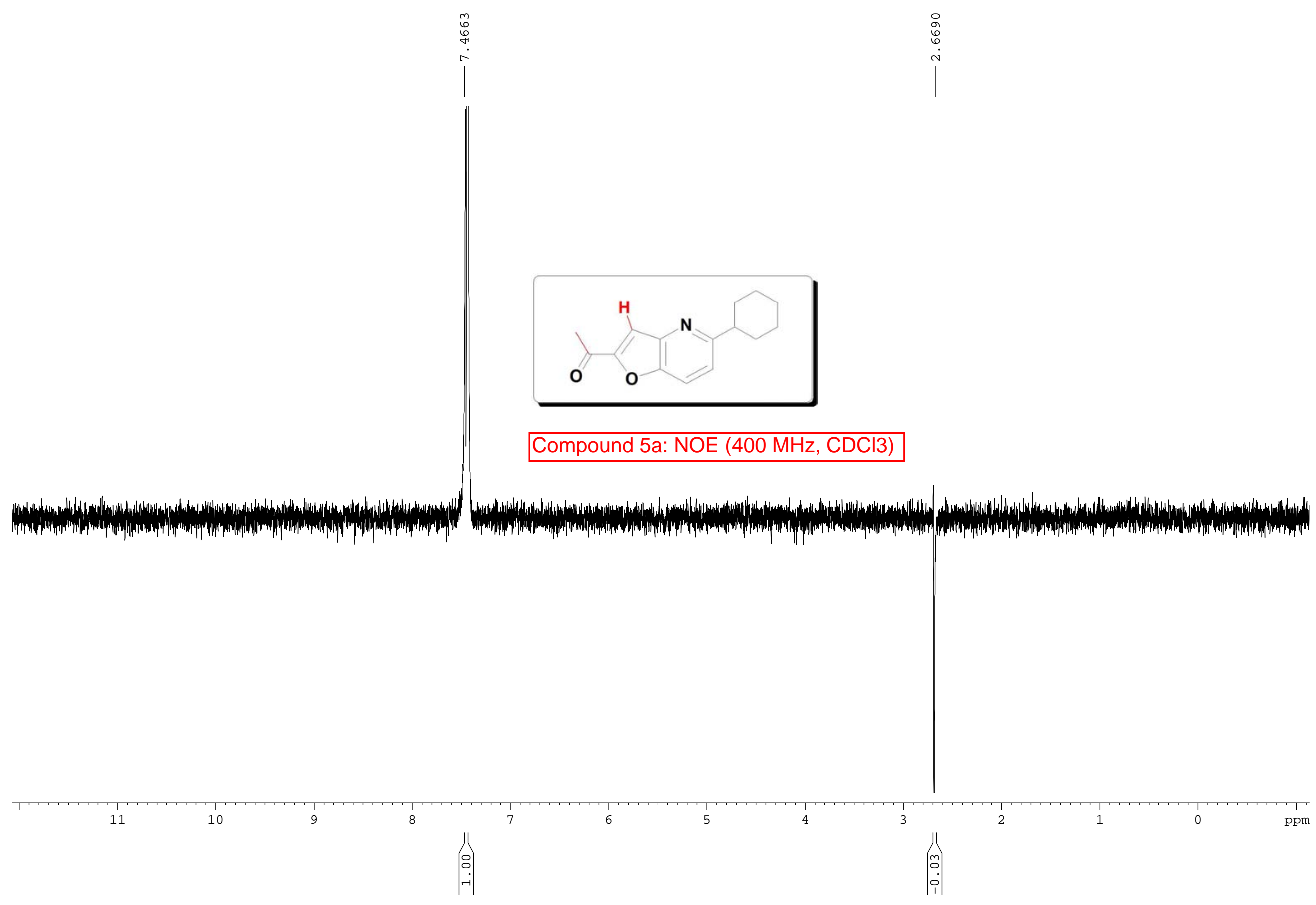




$$
-1
$$




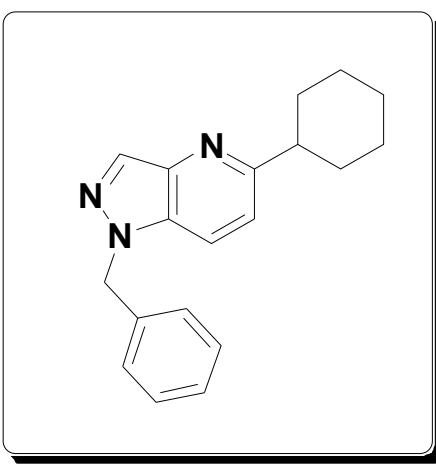

Compound 5b: 13C-NMR (100 MHz, CDCl3)

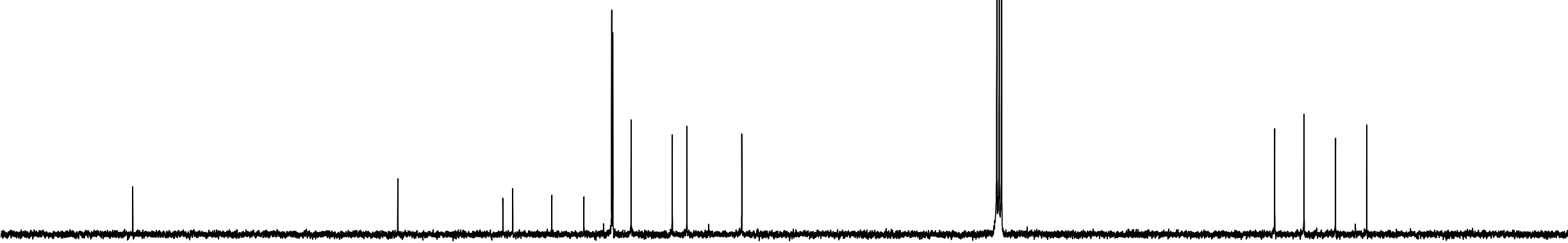




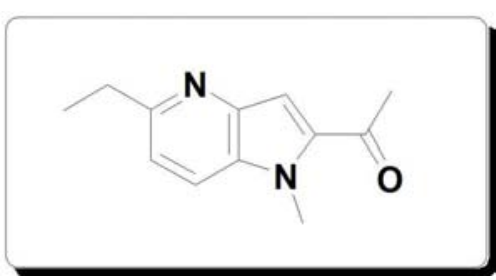

Compound 5c: 1H-NMR (400 MHz, CDCl3)
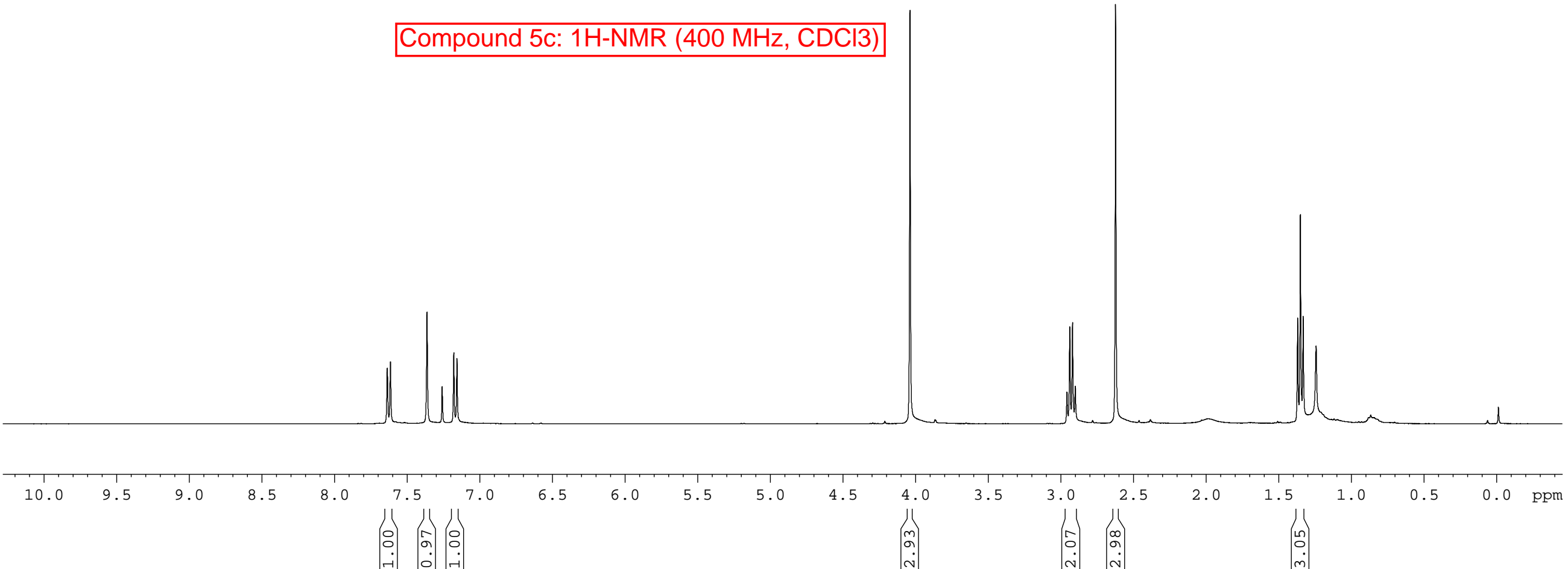


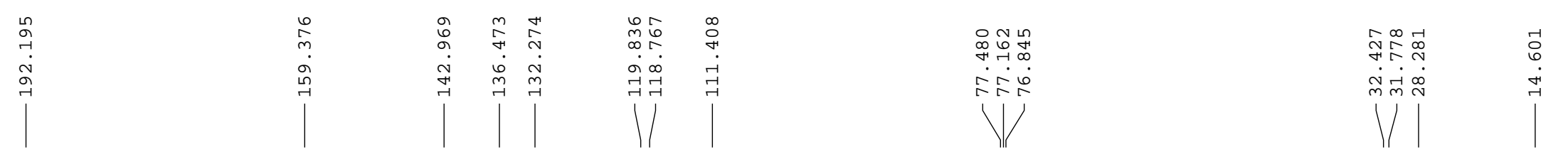
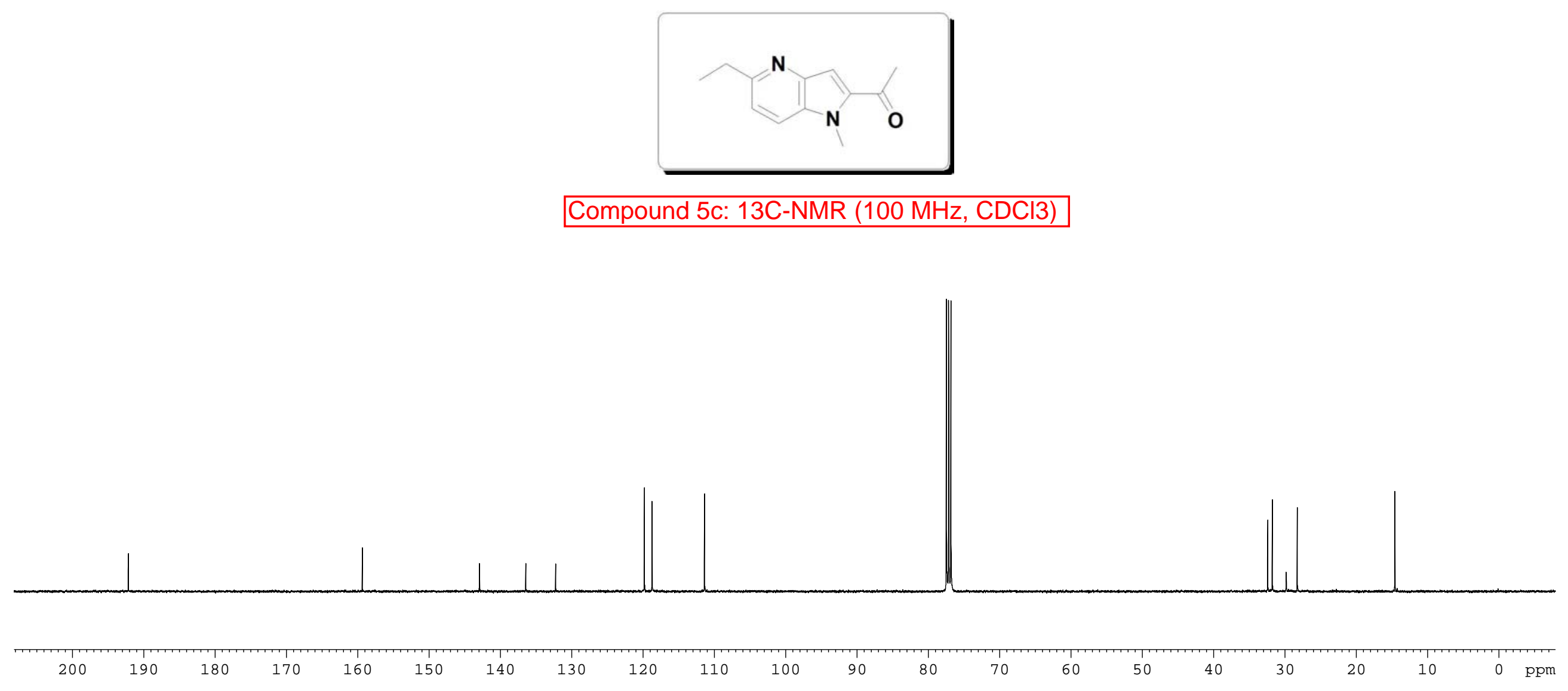
F2 - Acquisition Parameters

Date__ 20160404

$\begin{array}{ll}\text { Time } & 19.50 \\ \text { INSTRUM } & \text { spect }\end{array}$

spect
PROBHD $5 \mathrm{~mm} \mathrm{PABBO}$ BB-

$\begin{array}{lr}\text { PULPROG } & \text { selnogp } \\ \text { TD } & 65536\end{array}$

TD

SOLVENT

NS

DS

SWH

AQ

RG

DW

$\mathrm{DE}$

DCl3

$8223.685 \mathrm{~Hz}$ $0.125483 \mathrm{~Hz}$ $3.9846387 \mathrm{sec}$ 209.25

60.800 usec 6.50 use

$298.7 \mathrm{~K}$

$1.00000000 \mathrm{sec}$

$0.50000000 \mathrm{sec}$

$0.00020000 \mathrm{sec}$

$0.24879999 \mathrm{sec}$
1

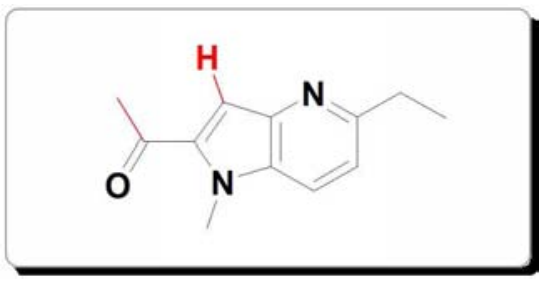

Compound 5c: NOE (400 MHz, CDCl3)

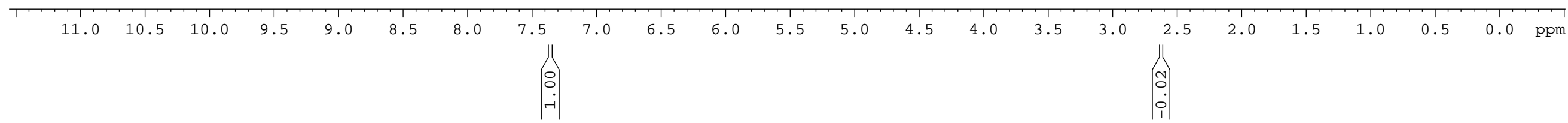




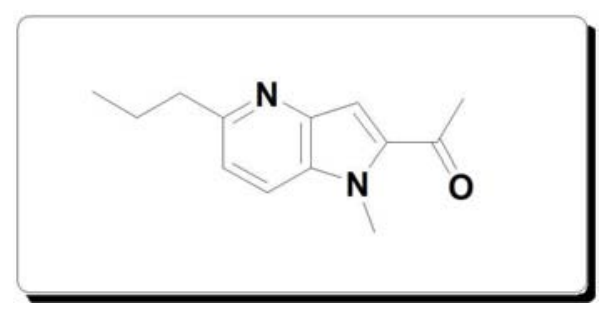

Compound 5d: 1H-NMR (400 MHz, CDCl3)

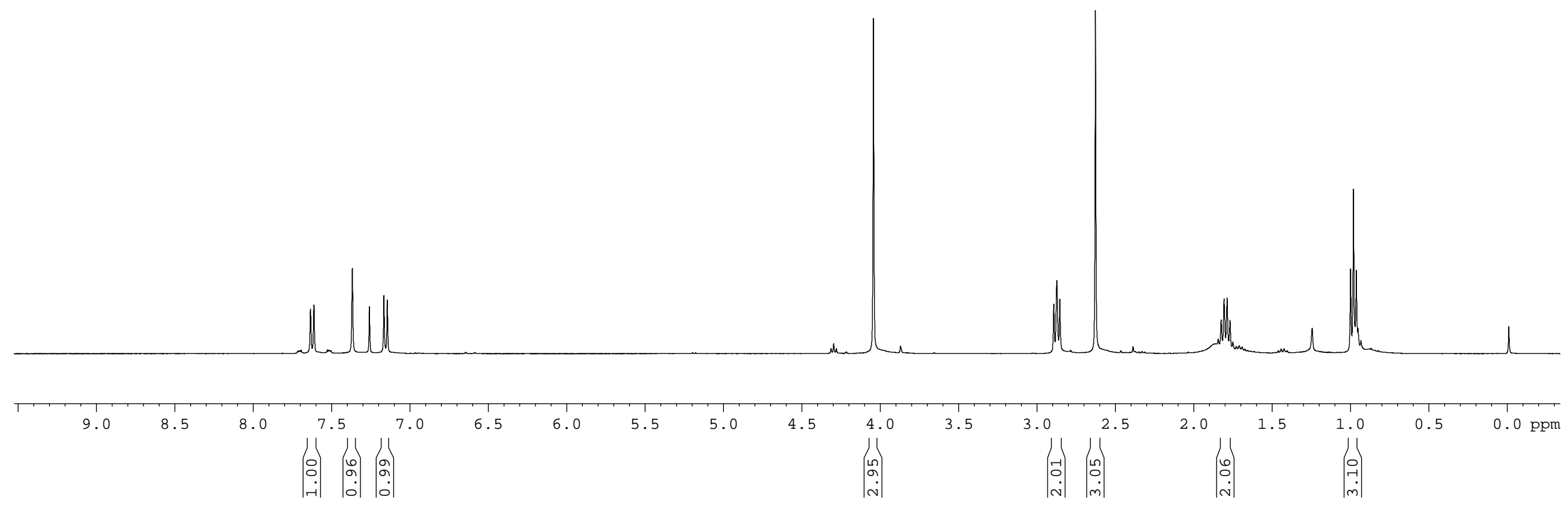




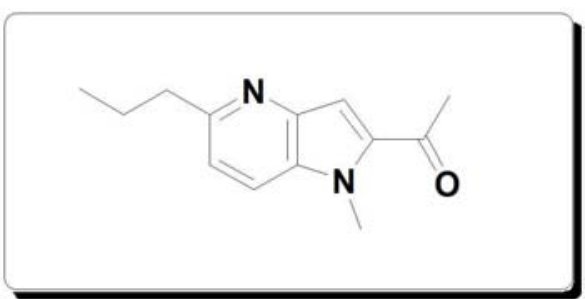

Compound 5d: 13C-NMR (100 MHz, CDCl3)

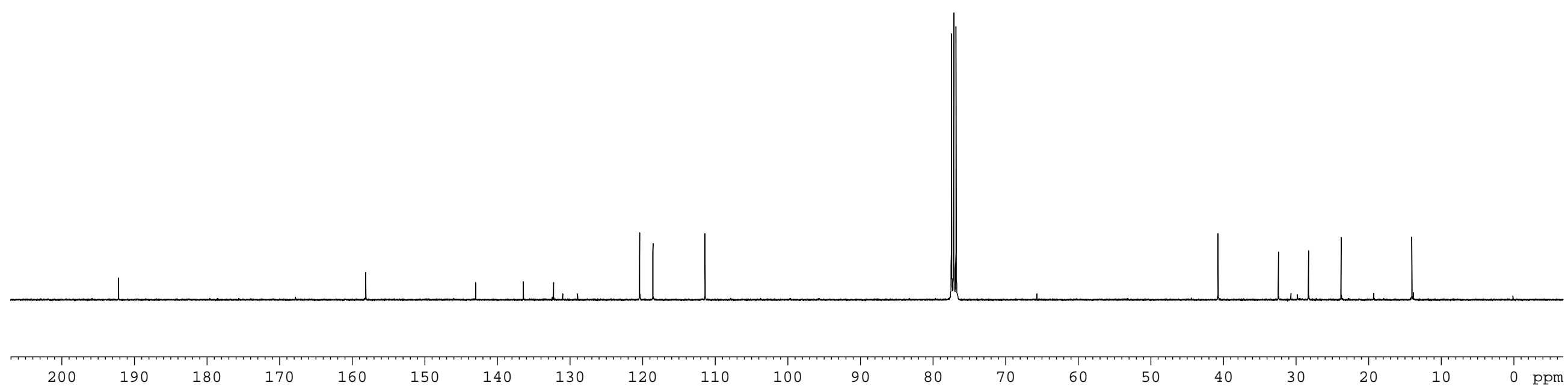




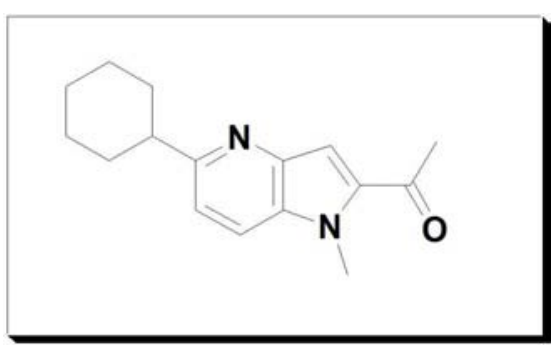

Compound 5e: 1H-NMR (400 MHz, CDCl3)

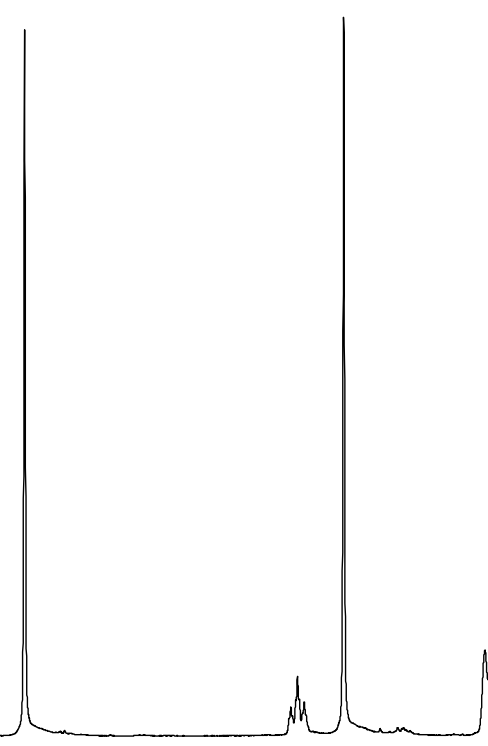

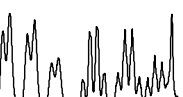

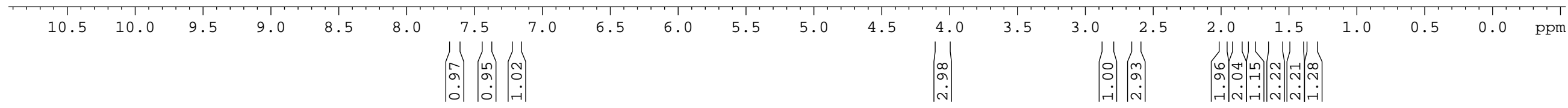




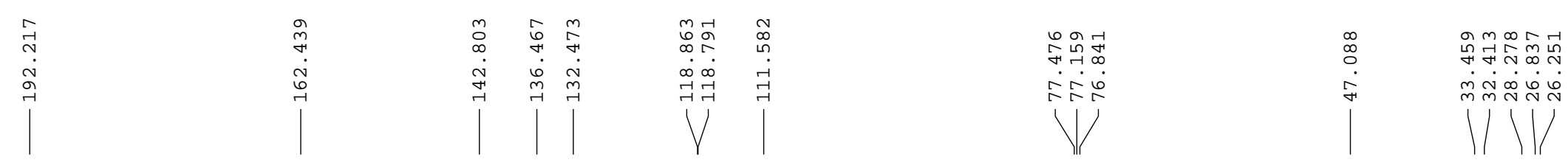

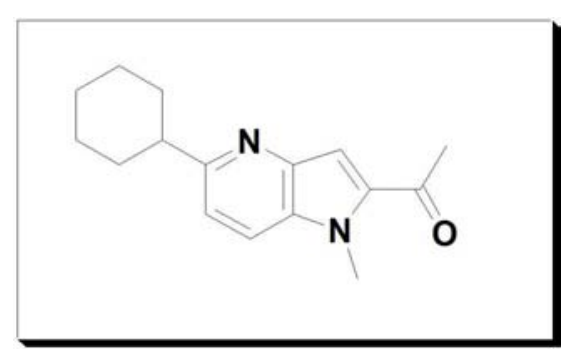

Compound 5e: 13C-NMR (100 MHz, CDCl3)

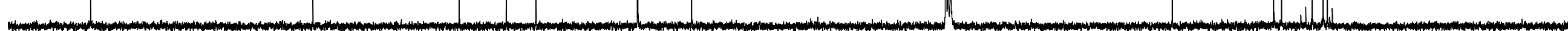

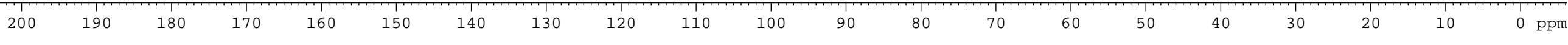




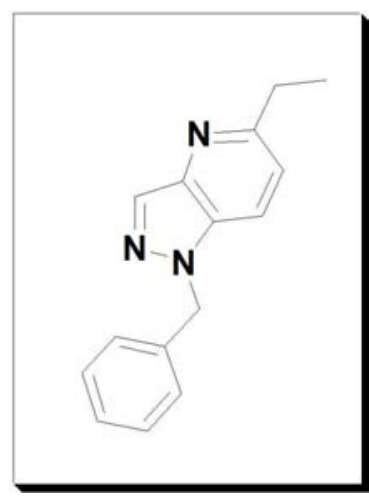

Compound 5f: 1H-NMR (400 MHz, CDCl3)

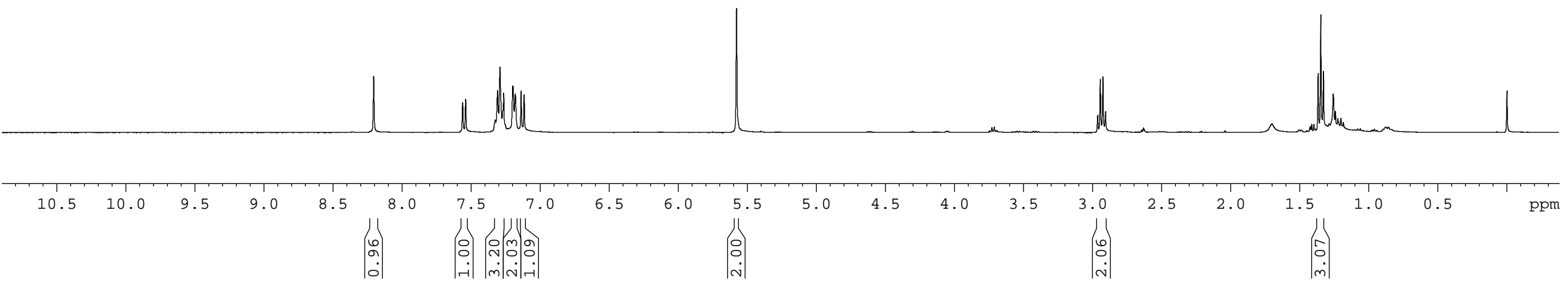




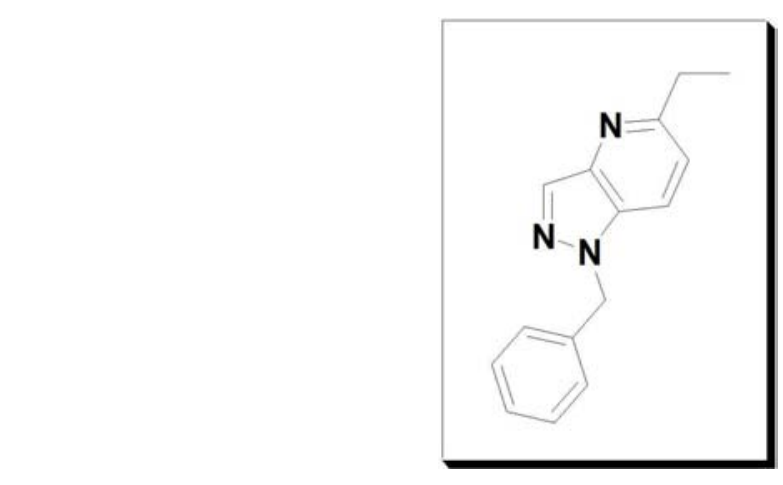

Compound 5f: 13C-NMR (100 MHz, CDCl3)

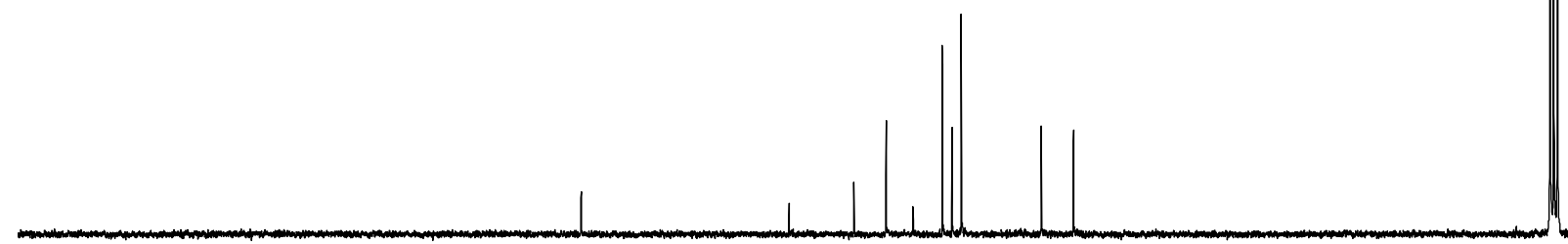


Current Data Parameters

NAME ly8-p68-P2-08132015

EXPNO

10

ROCNO

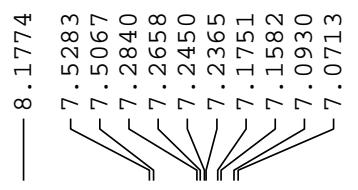

F2 - Acquisition Parameters

Date__ 20150813

Time 20.03

INSTRUM spect

5

zg30

TD $\quad 65536$

$\begin{array}{lr}\text { SOLVENT } & \text { CDC13 } \\ \text { NS } & 16\end{array}$

DS

SWH $\quad 8223.685 \mathrm{~Hz}$

$0.125483 \mathrm{~Hz}$

$\mathrm{AQ} \quad 3.9845889 \mathrm{se}$

RG 144.49

DW $\quad 60.800$ usec

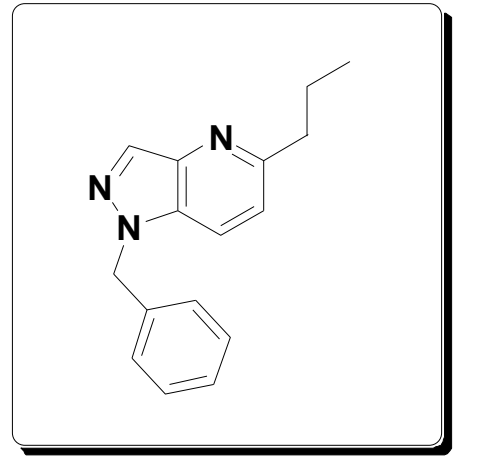

Compound 5g: 1H-NMR (400 MHz, CDCl3)

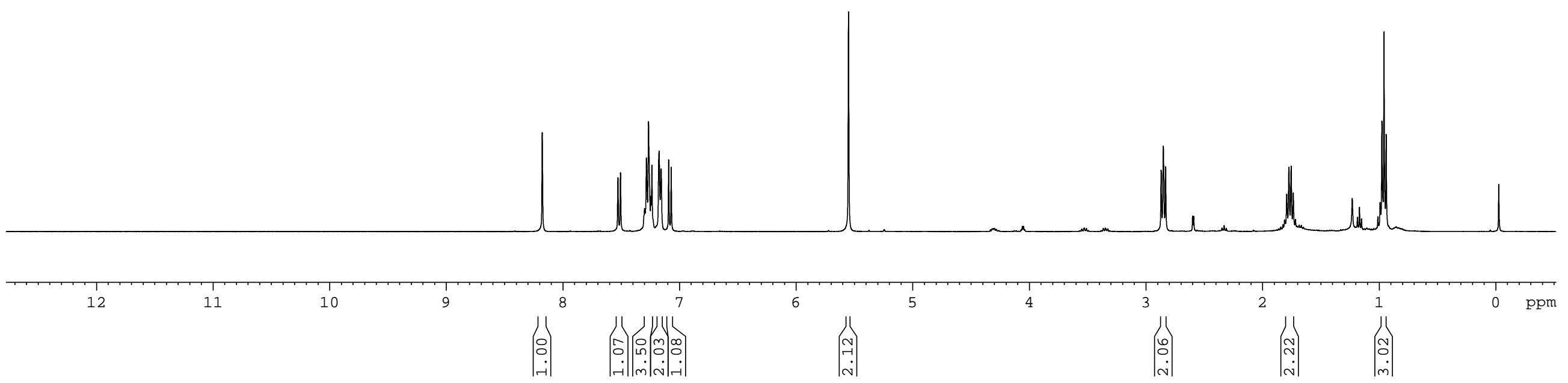




$$
\frac{1}{1+}
$$


Current Data Parameters

NAME ly8-p67-P1-150811-

EXPNO

PROCNO

F2 - Acquisition Parameters

Date_ $\quad 20150811$

Time $\quad 12.04$

STRUM spect

$5 \mathrm{~mm}$ PABBO BB

PULPROG $\quad \mathrm{zg} 30$

TD 65536

SOLVENT CDCl3

NS

DS

SWH

FIDRES

$\mathrm{AQ}$

RG

DW
$8012.820 \mathrm{~Hz}$
$0.122266 \mathrm{~Hz}$
$4.0894465 \mathrm{sec}$
32.58
62.400 usec

62.400 usec

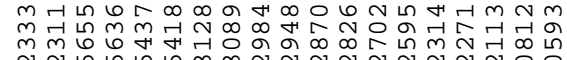

.

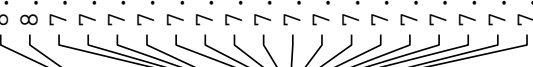

$\rightarrow$ N

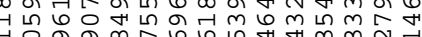

(N)
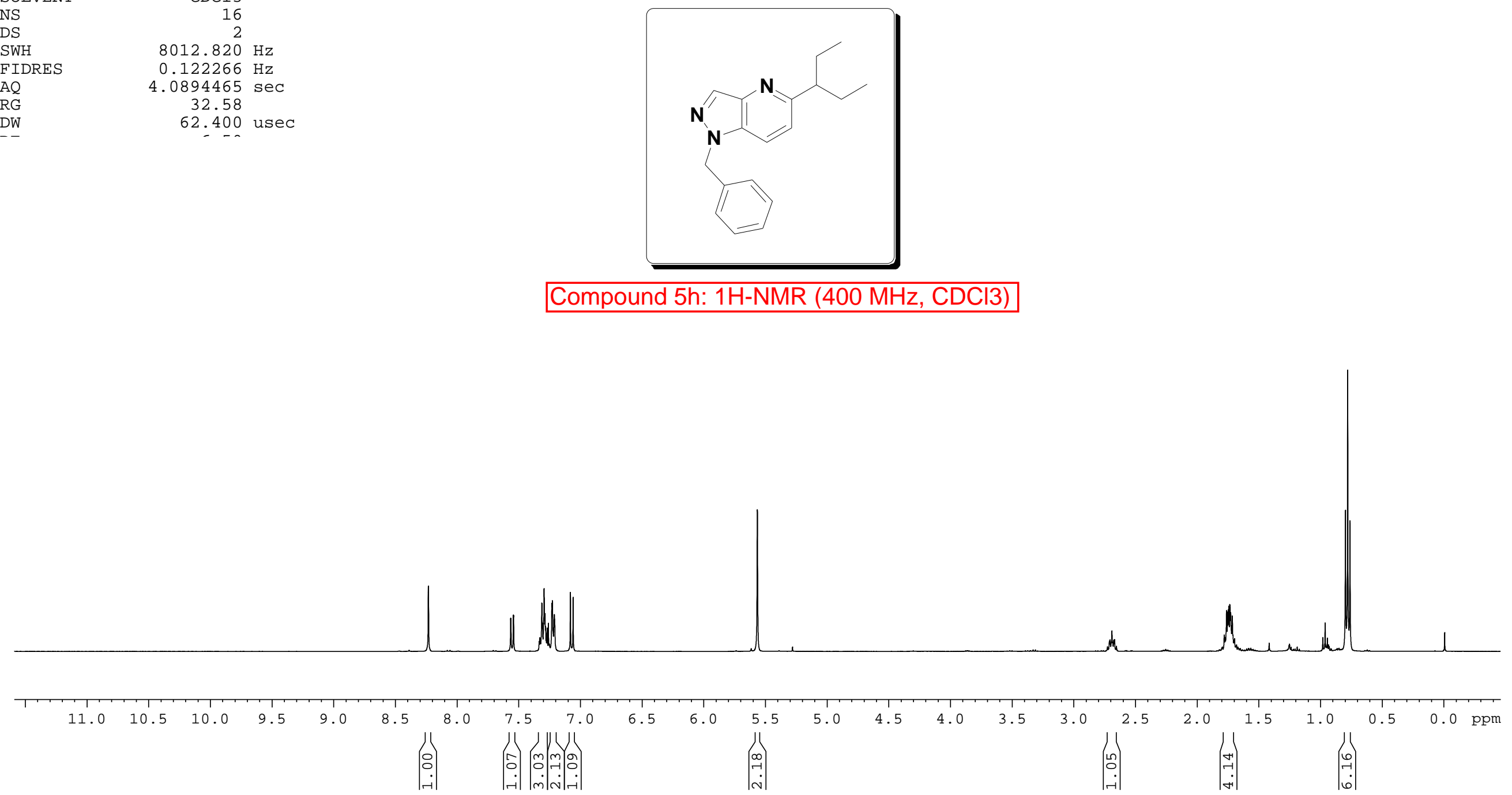


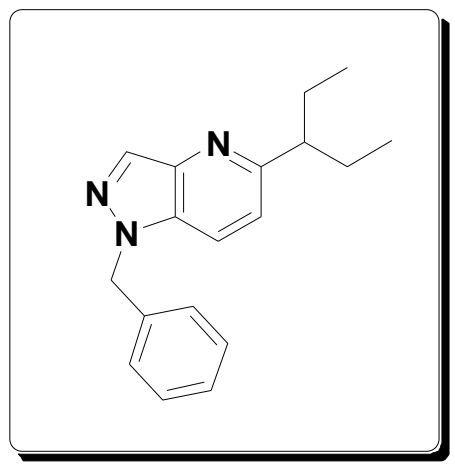

Compound 5h: 13C-NMR (100 MHz, CDCl3)

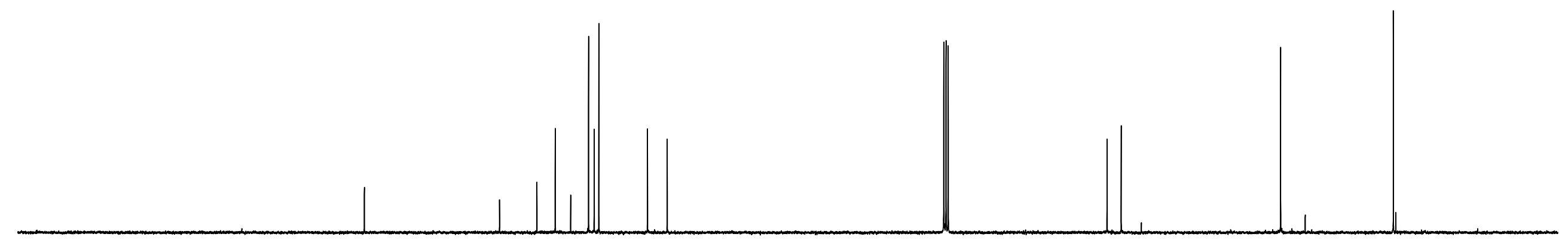




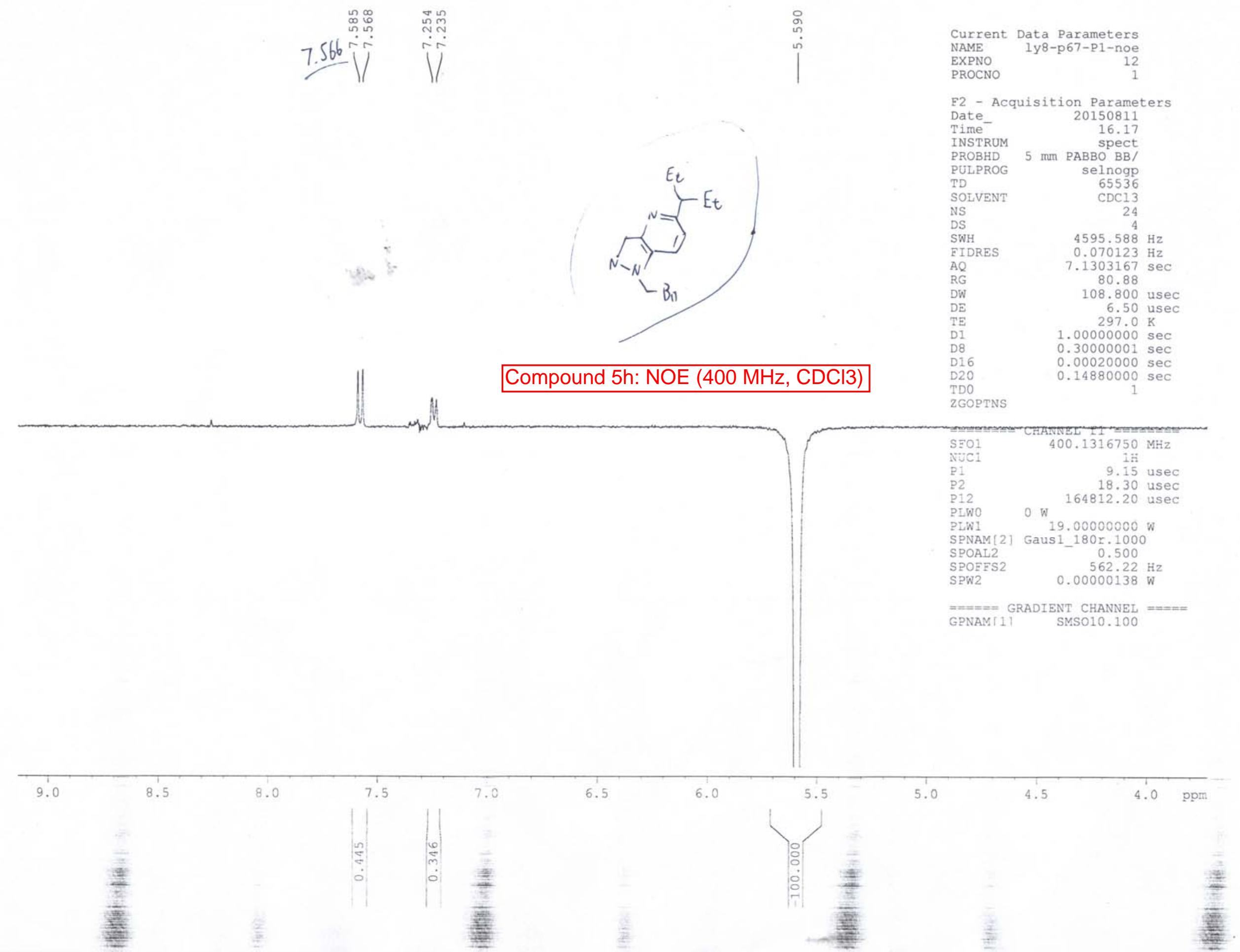


Current Data Parameters

NAME 1y9-p11-twice-160415-

EXPNO

E2 - Acquisition Parameter

Date_ 20160415

Time $\quad 15.42$

PROBHD $5 \mathrm{~mm}$ PABBO BB/

PULPROG $\quad$ zg30

TD 65536

NS

DS

16
2
$2 .$.
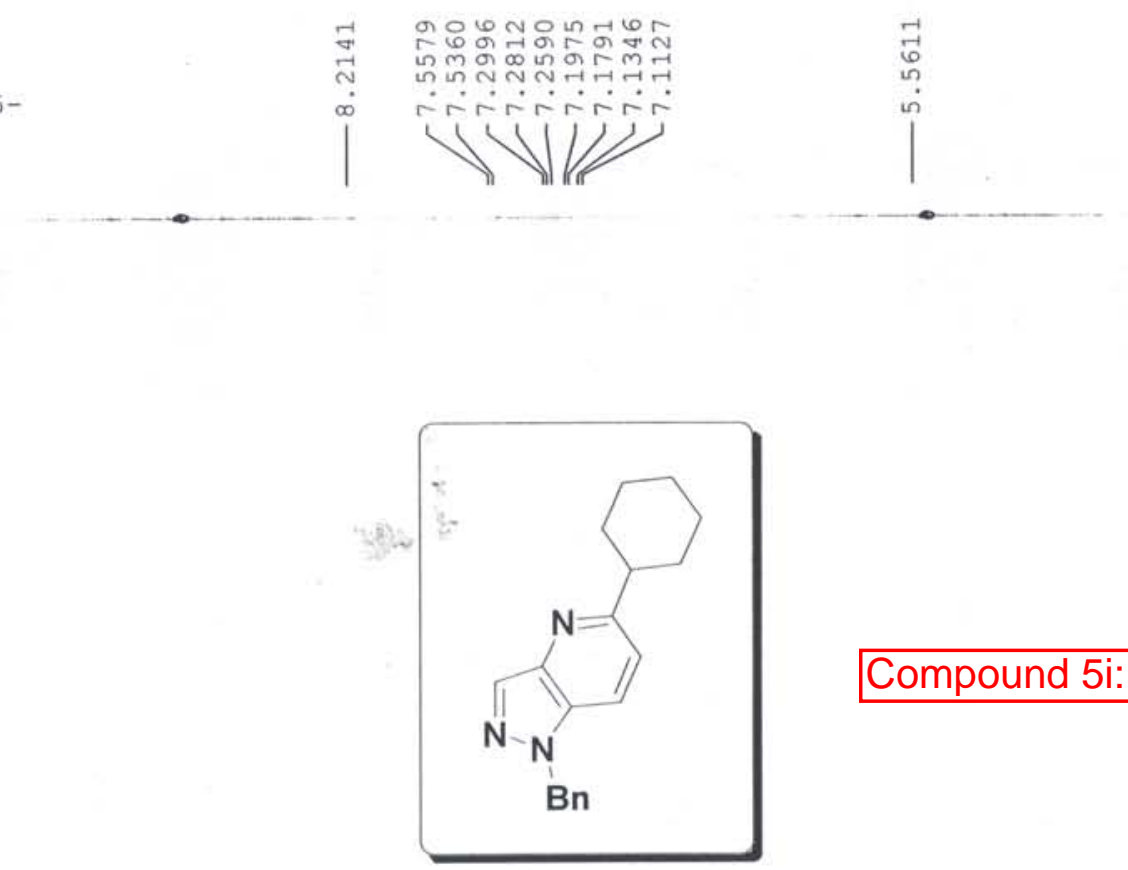

ONAMNMMIn

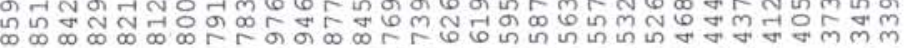
बल
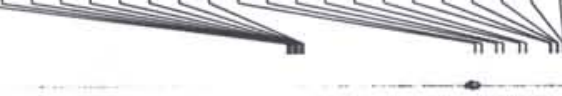

\section{Compound 5i: 1H-NMR (400 MHz, CDCl3)}

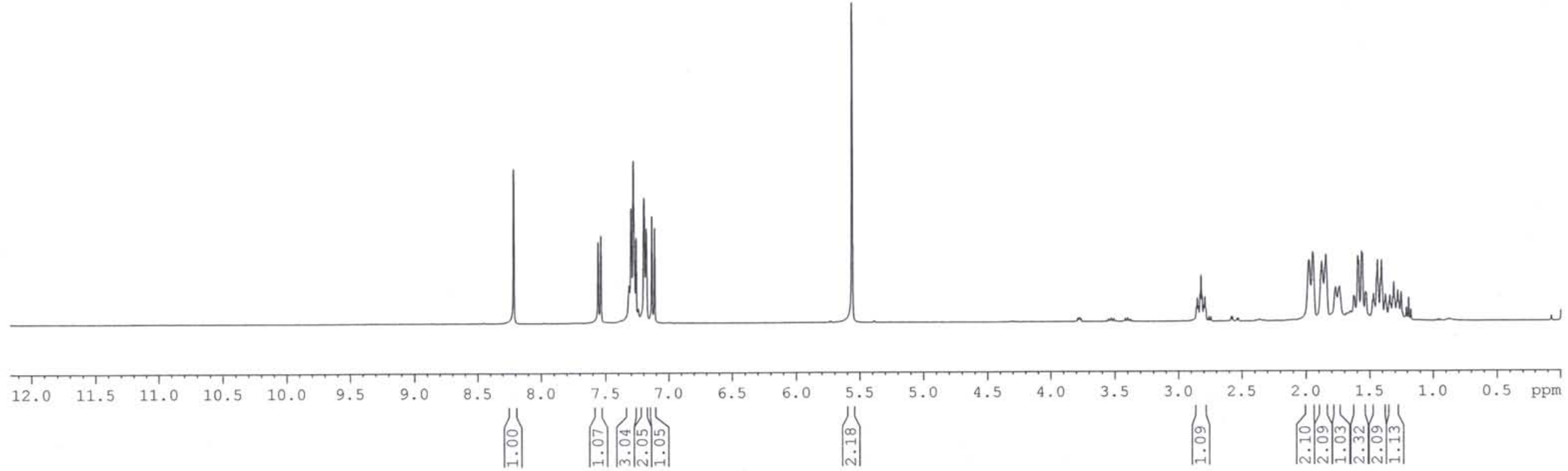




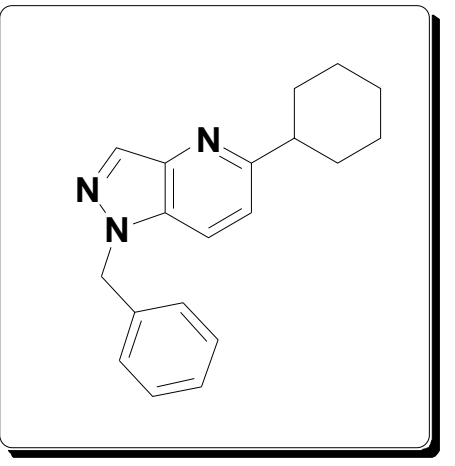

Compound 5i: 13C-NMR (100 MHz, CDCl3)

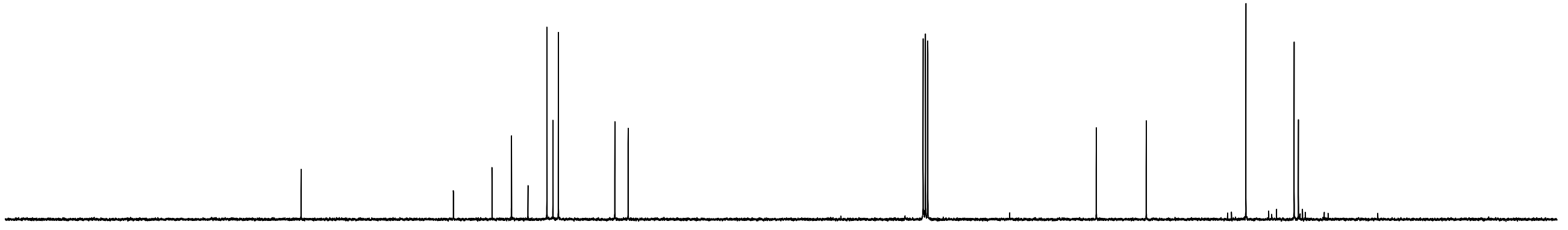



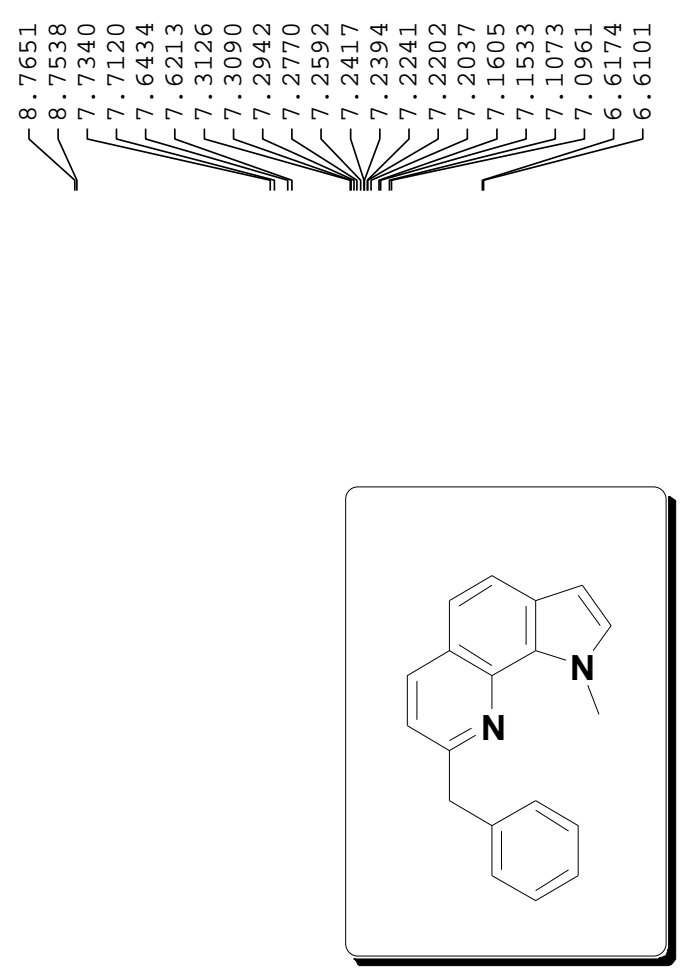

Compound 5j: 1H-NMR (400 MHz, CDCl3)
Current Data Parameters ly8-p69-PH-150817 PROCNO

F2 - Acquisition Parameters Date__ 20150817

$\begin{array}{lr}\text { Time } & 12.19\end{array}$

INSTRUM spect

PROBHD $5 \mathrm{~mm} \mathrm{PABBO} \mathrm{BB} /$

$\mathrm{zg} 30$
65536

TD

NS

DS

FIDRES

$\mathrm{AQ}$

RG
$\mathrm{CDC} 13$

$8012.820 \mathrm{~Hz}$

$0.122266 \mathrm{~Hz}$ $4.0894465 \mathrm{sec}$ 89.81 62.400 usec

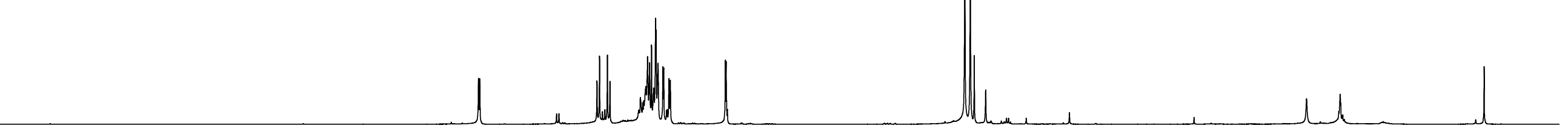

$120 \begin{array}{lll}1 & 1 & 1\end{array}$

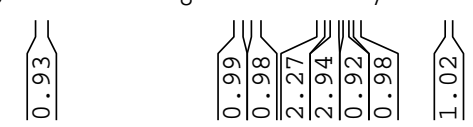

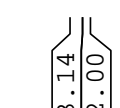




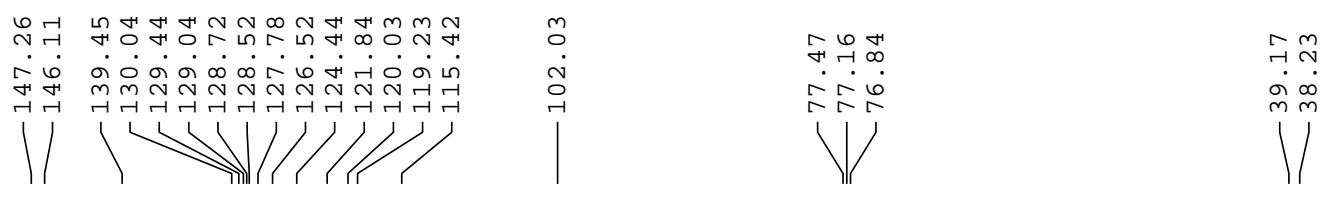

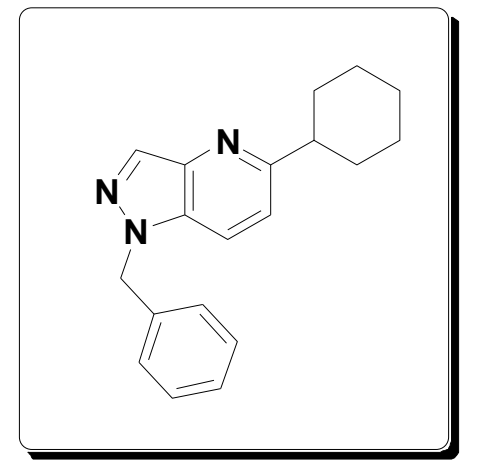

Compound 5j: 13C-NMR (100 MHz, CDCl3)

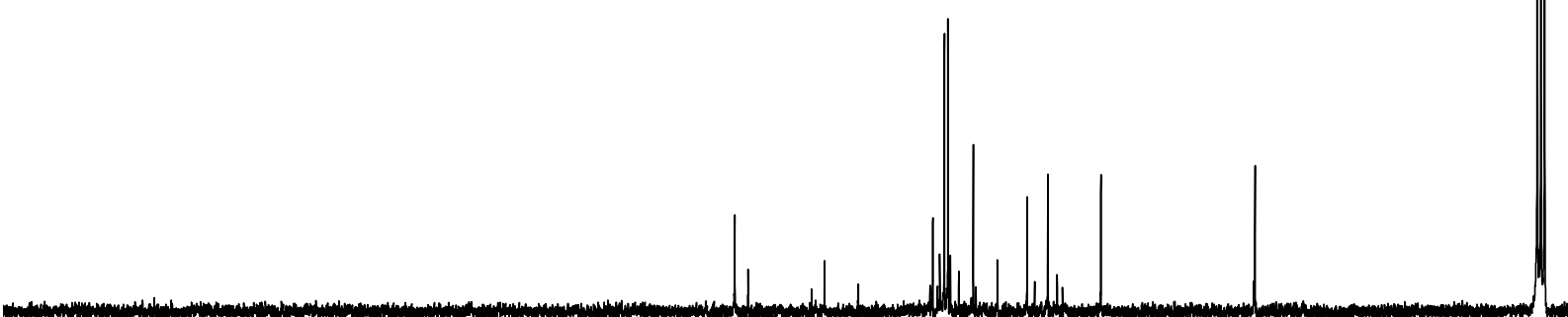


F2 - Acquisition Parameters

Date_ 20150804

Time 22.24

INSTRUM spect

PROBHD $5 \mathrm{~mm}$ PABBO BB/

PULPROG $\quad$ zg30

TD

NS $\quad 16$

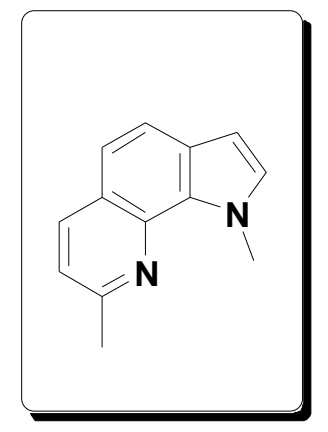

Compound 5k: 1H-NMR (400 MHz, CDCl3)

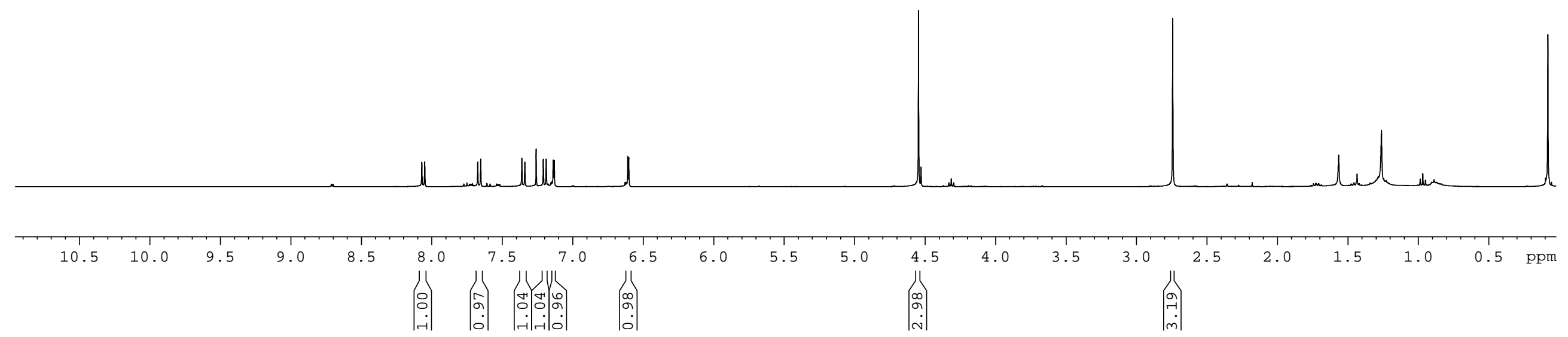




$$
-1
$$


F2 - Acquisition Parameters Date_ 20150804 Time 10.27

INSTRUM spect

PROBHD $5 \mathrm{~mm}$ PABBO BB/

PULPROG zg30

65536

TD

$\mathrm{CDCl}$

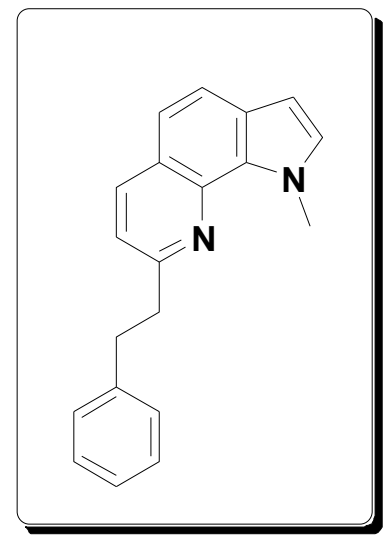

Compound 5l: 1H-NMR (400 MHz, CDCl3)
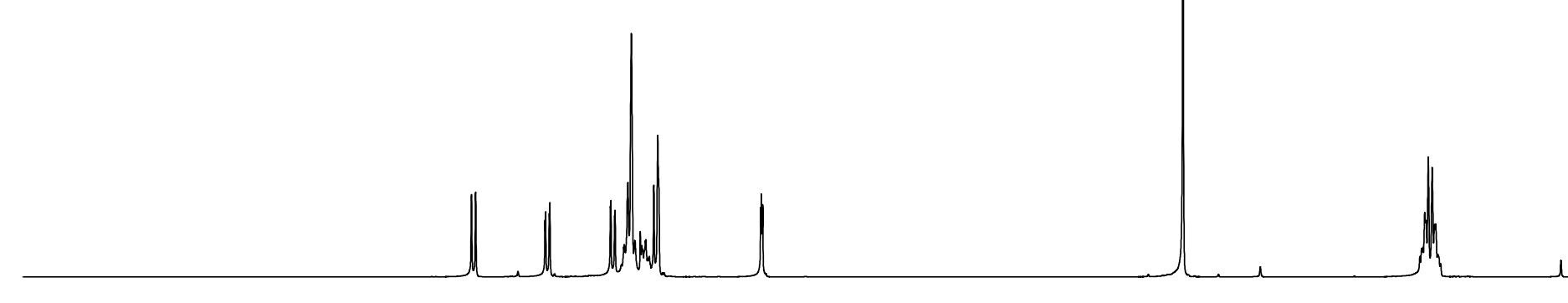

$\mu$

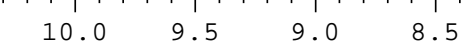

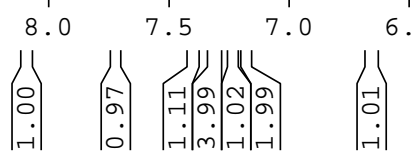

$\left|\begin{array}{c}4 \\ 1 \\ 0 \\ 0\end{array}\right|$

$4.0 \quad 3.5$ 


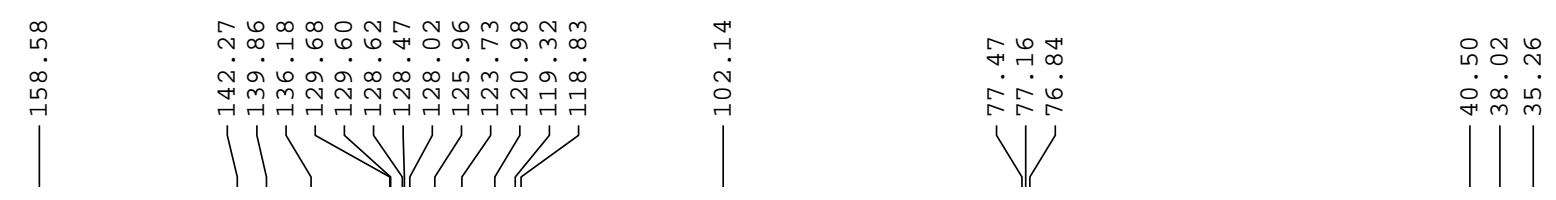
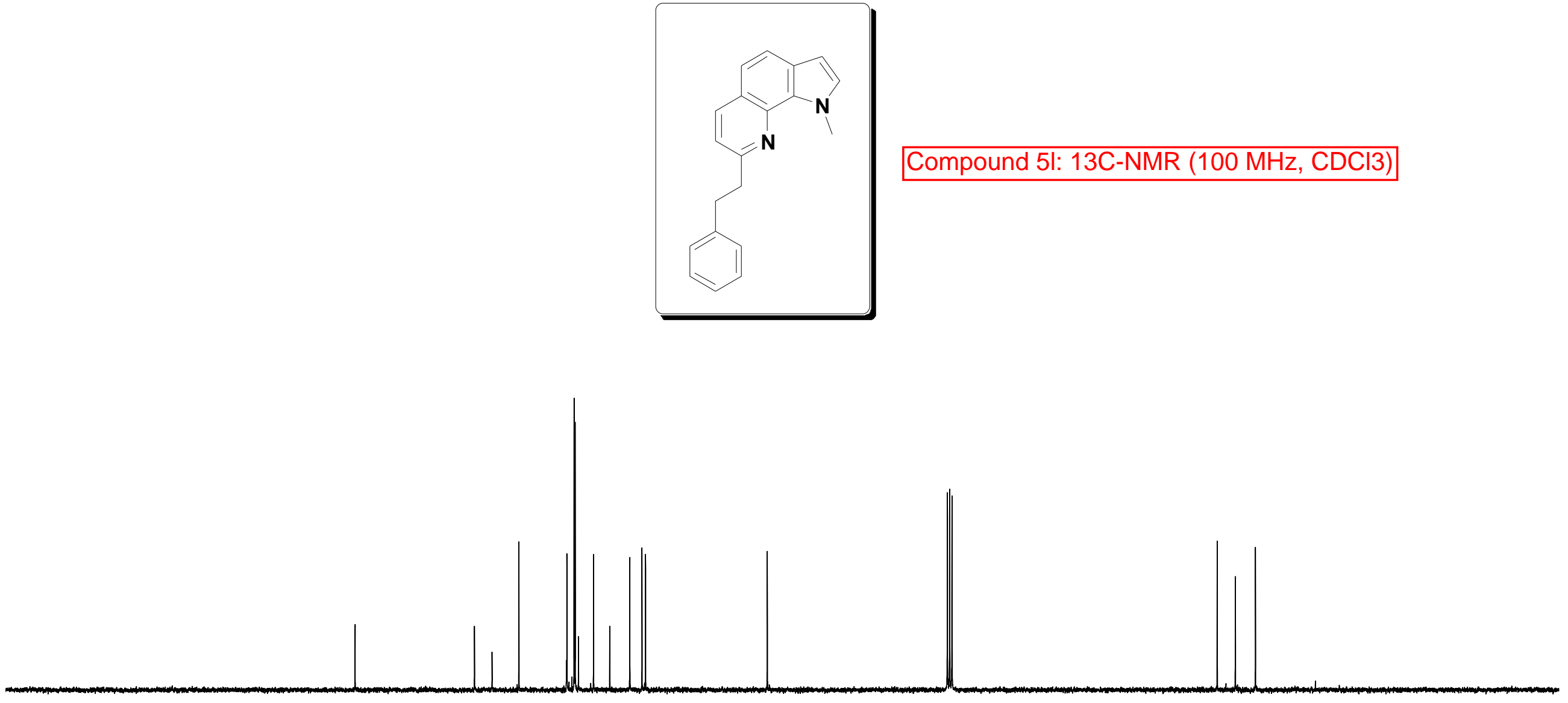

\begin{tabular}{|c|c|c|c|c|c|c|c|c|c|c|c|c|c|c|c|c|c|c|}
\hline 200 & 190 & 180 & 170 & 160 & 150 & 140 & 130 & 120 & 110 & 100 & 90 & 80 & 70 & 60 & 50 & 40 & 30 & $0 \mathrm{ppm}$ \\
\hline
\end{tabular}



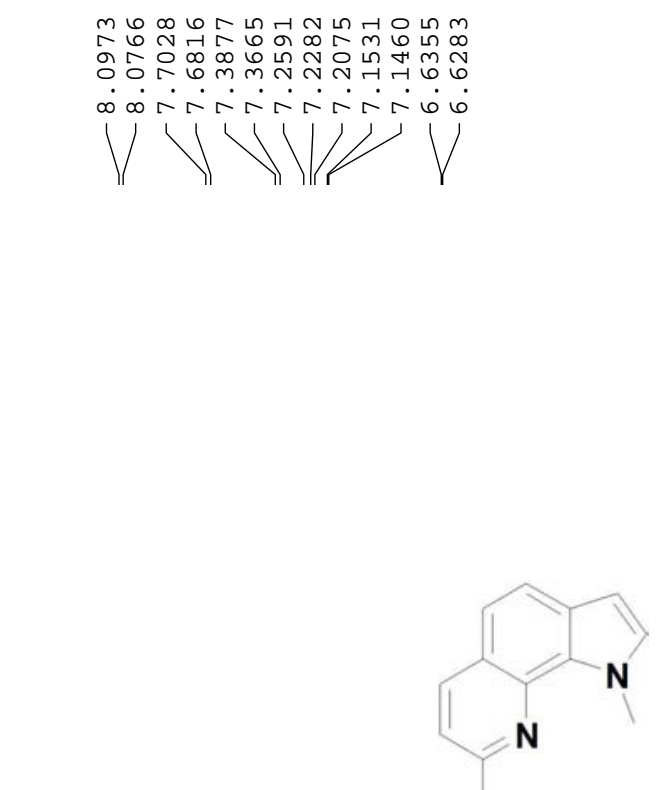

Compound 5m: 1H-NMR (400 MHz, CDCl3)

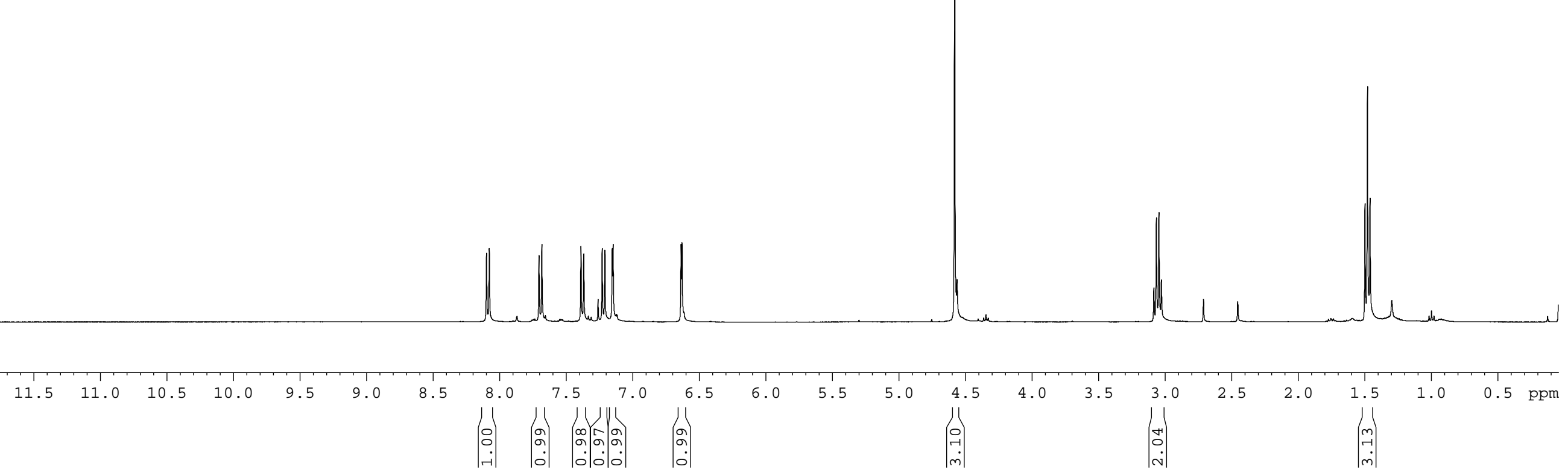


0
7
7
0
8
$i$
1

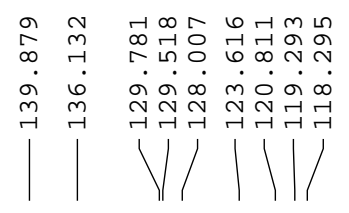

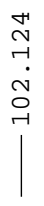

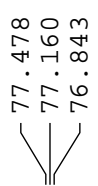

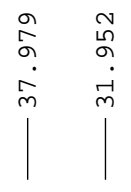

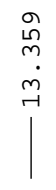

Compound 5m: 13C-NMR (100 MHz, CDCl3)

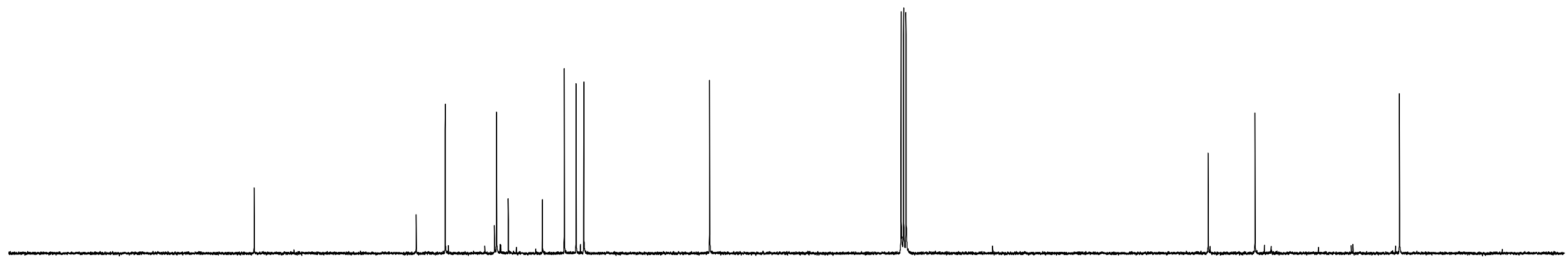

190

180

170

130

120

110

100

90

70

60

50

40

30

20

10

0

ppm 


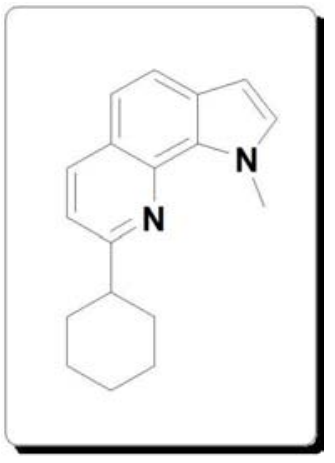

Compound 5n: 1H-NMR (400 MHz, CDCl3)

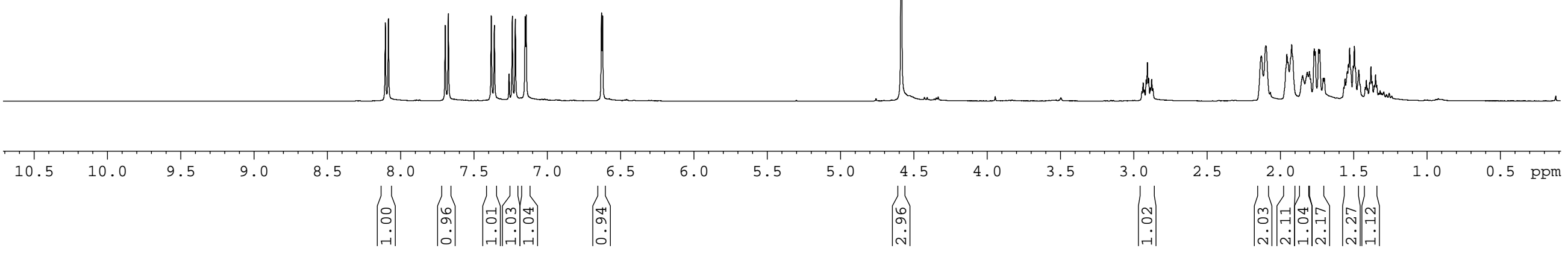




$$
\frac{1}{1+1}
$$




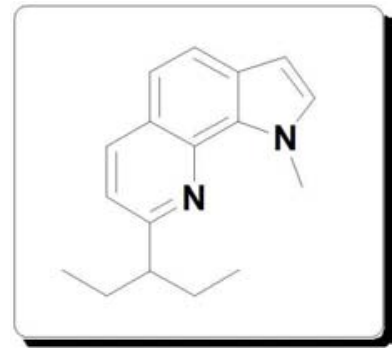

Compound 5o: 1H-NMR (400 MHz, CDCl3)
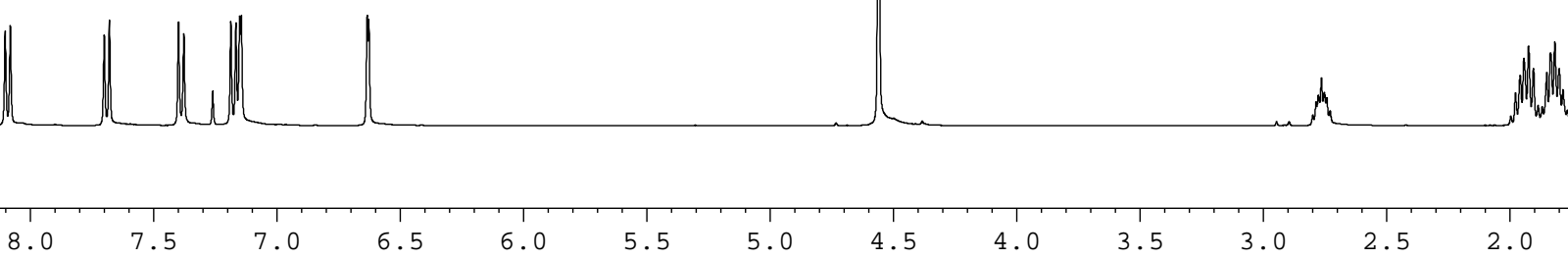

5.0

$\mid \begin{array}{ll}2.5 \\ 0 \\ 0\end{array}$
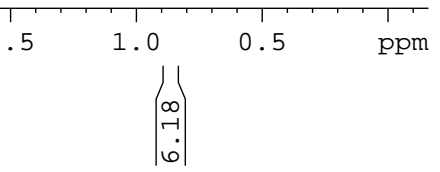


|

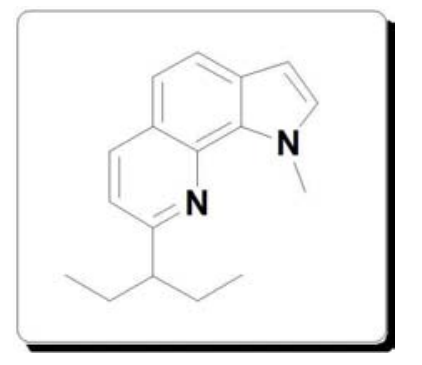

Compound 5o: 13C-NMR (100 MHz, CDCl3)

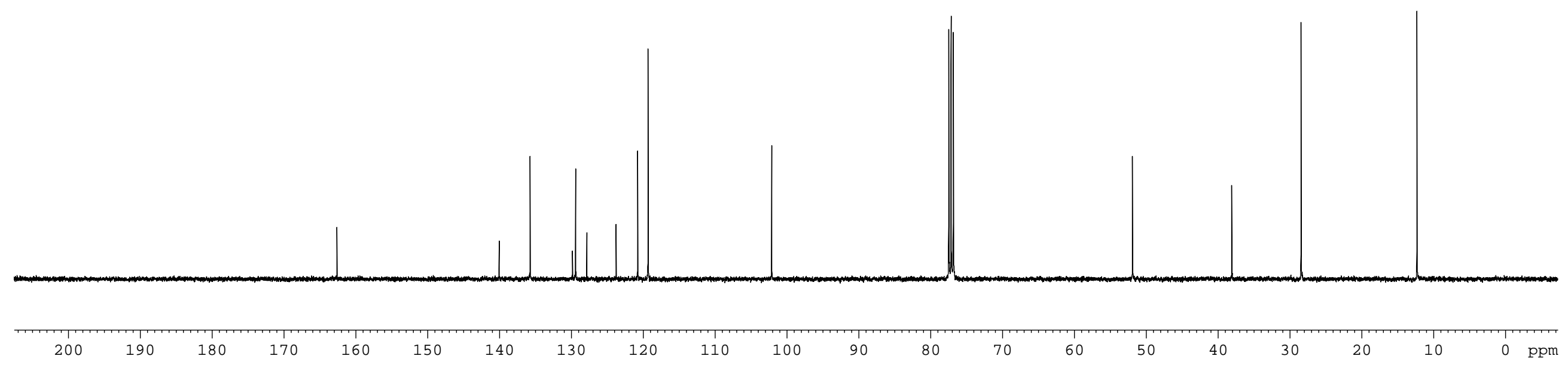



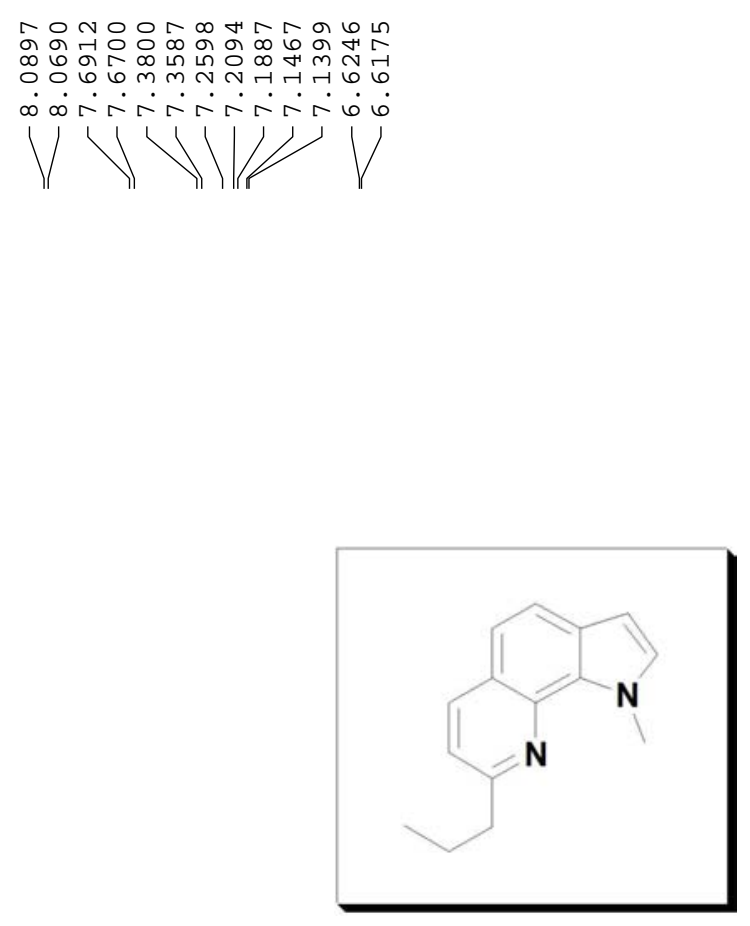

Compound 5p: 1H-NMR (400 MHz, CDCl3)

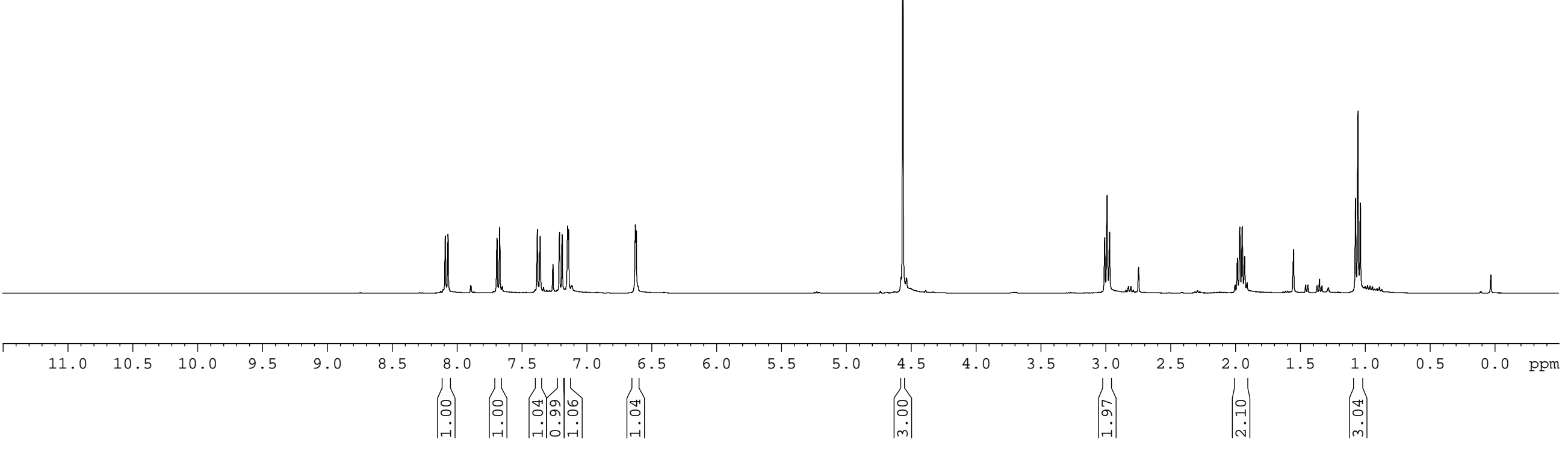




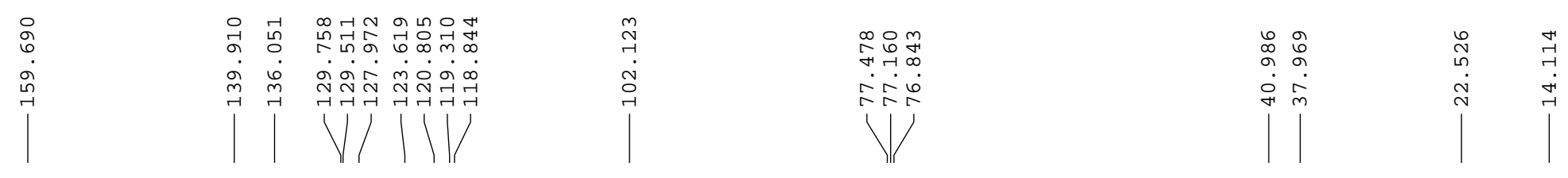

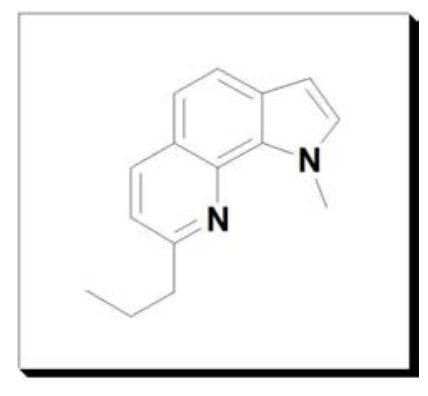

Compound 5p: 13C-NMR (100 MHz, CDCl3)

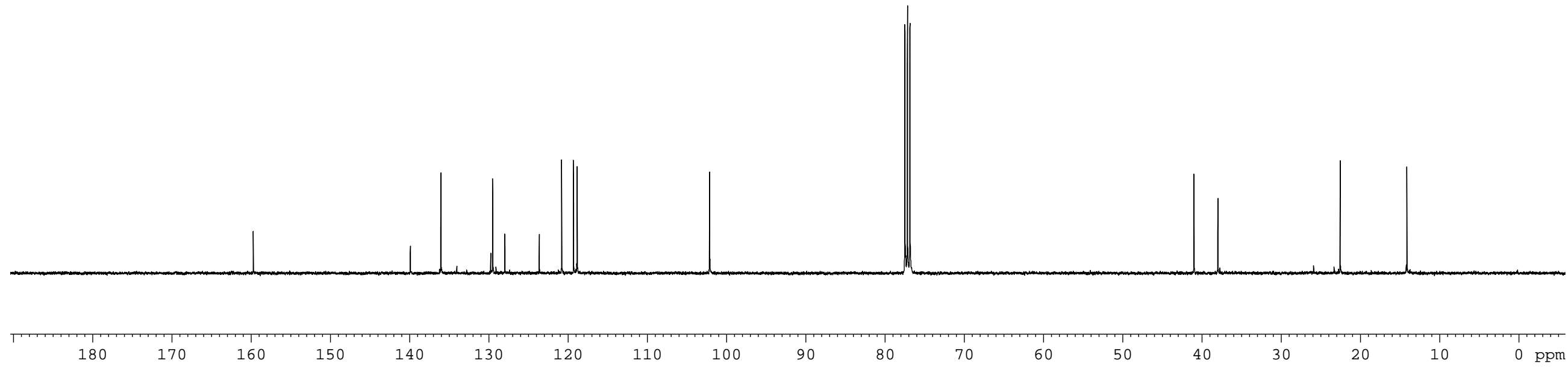


Current Data Parameters

NAME ly8-p64-3P-150808-

EXPNO

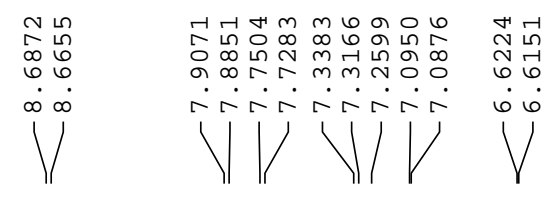

F2 - Acquisition Parameters

Date_ 20150808

Time $\quad 17.34$

spect
INSTRUM

PULPROG 5 mm PABBO

SOLVENT

NS

DS

FID

$\mathrm{AQ}$

RG

DW

$$
\begin{array}{r}
\text { zg30 } \\
65536 \\
\text { CDC13 } \\
16 \\
2 \\
8012.820 \mathrm{~Hz} \\
0.122266 \mathrm{~Hz} \\
4.0894465 \mathrm{sec} \\
128.61 \\
62.400 \text { usec }
\end{array}
$$

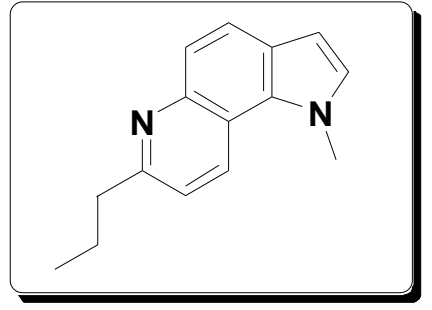

Compound 5q: 1H-NMR (400 MHz, CDCl3)

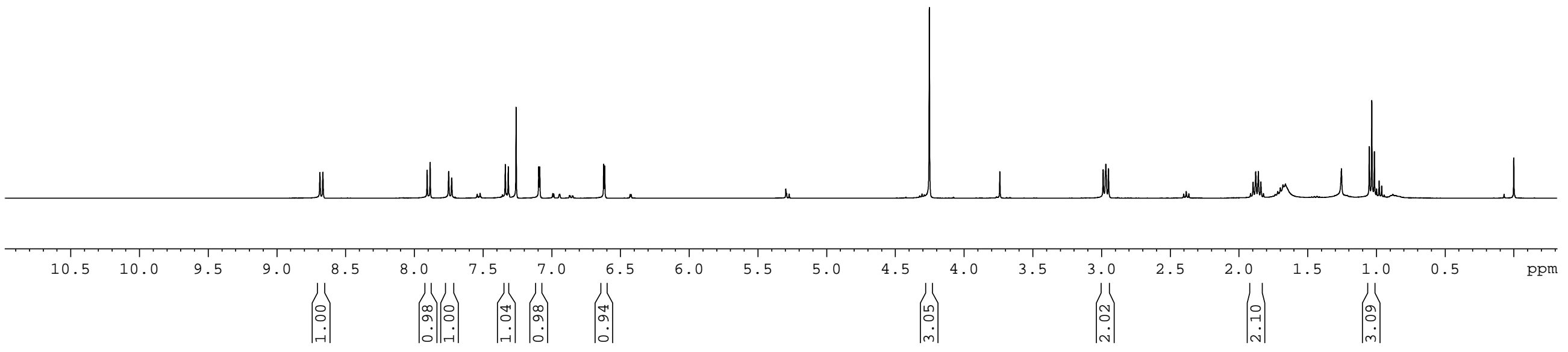




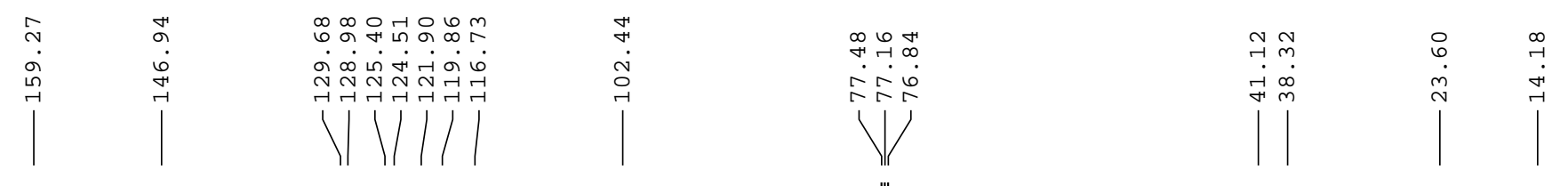




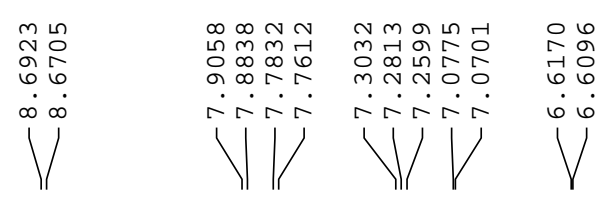

$\stackrel{\substack{n \\ m}}{\stackrel{\sigma}{\pi}}$
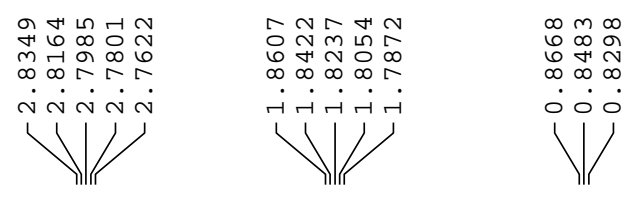

Current Data Parameters

NAME ly8-150805-

EXPNO

10
1

F2 - Acquisition Parameters

Date_ 20150805

$\begin{array}{lr}\text { Time } & 22.07\end{array}$

spect
INSTRUM $5 \mathrm{~mm} \mathrm{PABBO} \mathrm{BB} /$

PULPROG
zg30

TD 65536

SOLVENT CDCl3

NS

DWH

$\quad 8012.820 \mathrm{~Hz}$

AIDRES $\quad 0.122266 \mathrm{~Hz}$

AQ $\quad 4.0894465 \mathrm{sec}$

$\begin{array}{lr}\text { RG } & 57.28 \\ \text { DW } & 62.400 \text { usec }\end{array}$

$$
\text { -. }
$$

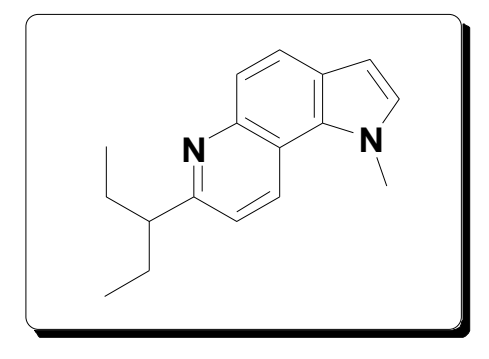

Compound 5r: $1 \mathrm{H}-\mathrm{NMR}(400 \mathrm{MHz}, \mathrm{CDCl})$
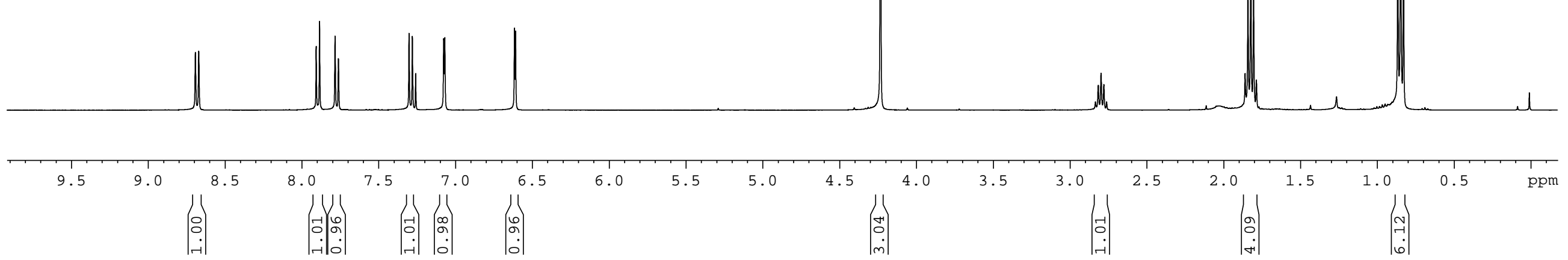


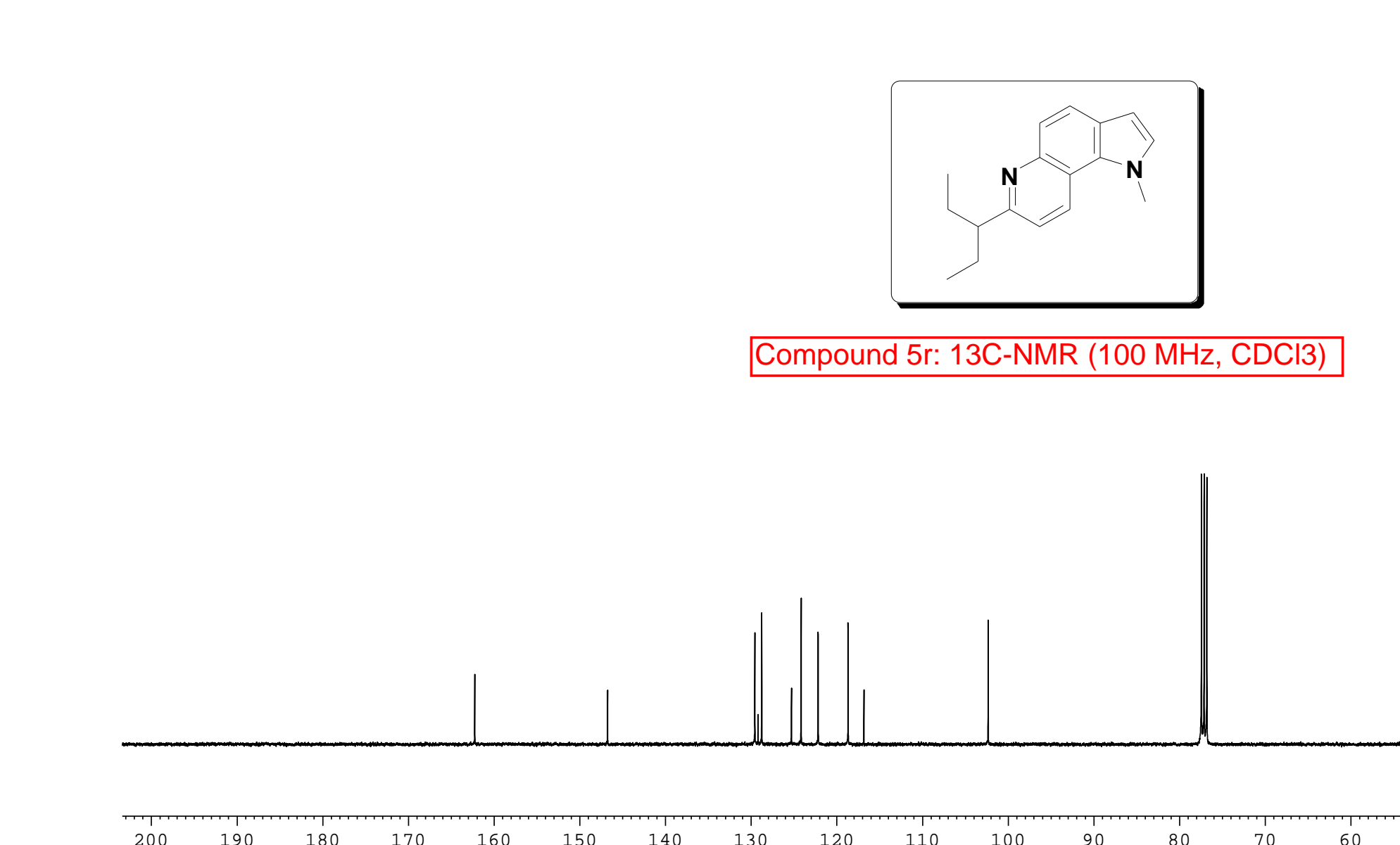


Current Data Parameters

NAME

$-150805$

PROCNO

10

F2 - Acquisition Parameters

Date_ 20150806

Time $\quad 0.06$

spect
PROBHD $5 \mathrm{~mm} \mathrm{PABBO}$ BB/

PROBHD $5 \mathrm{~mm}$ PABBO BB/
PULPROG

PULPROG $\quad$ zg30

TD 65536

SOLVENT

DS
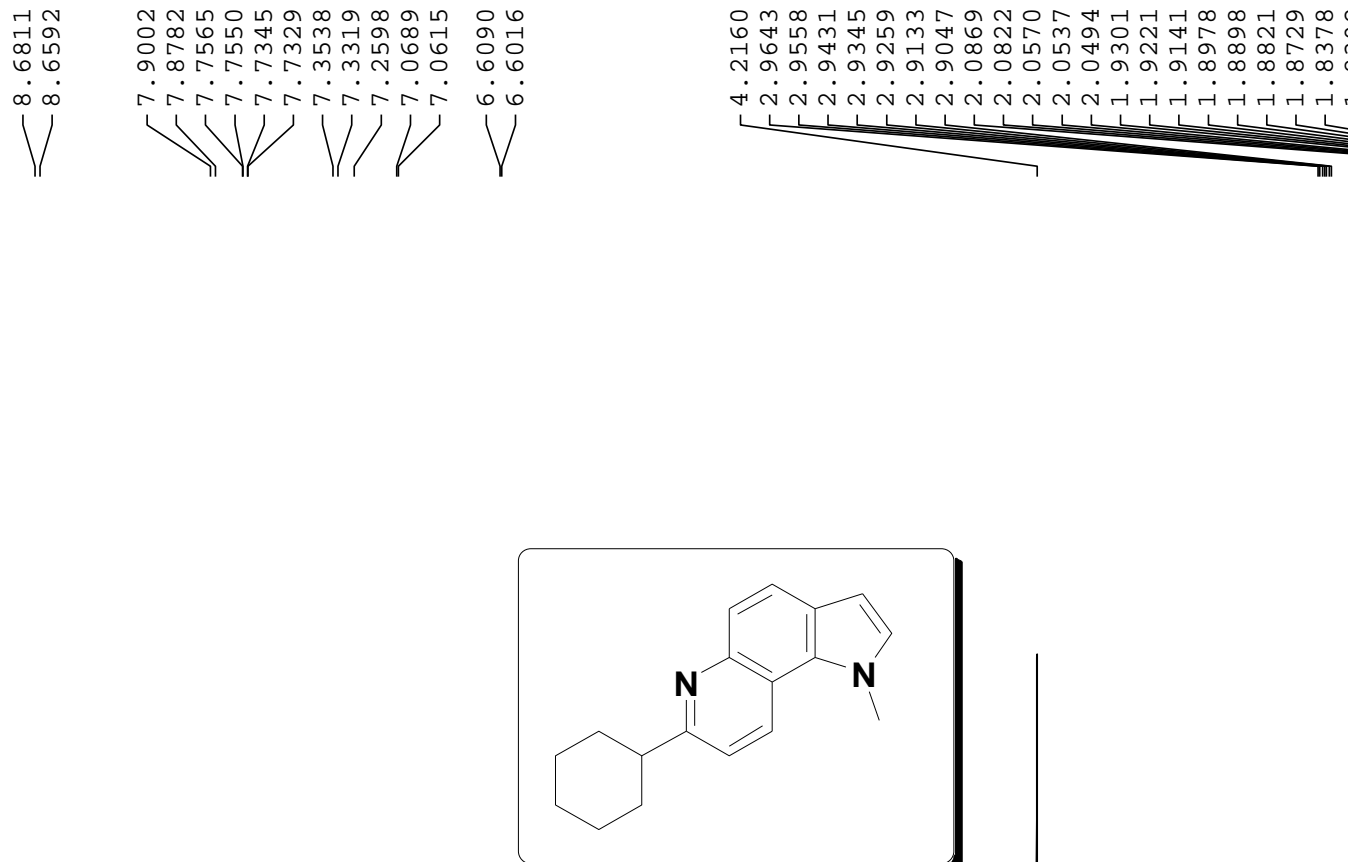

Compound 5s: 1H-NMR (400 MHz, CDCl3)

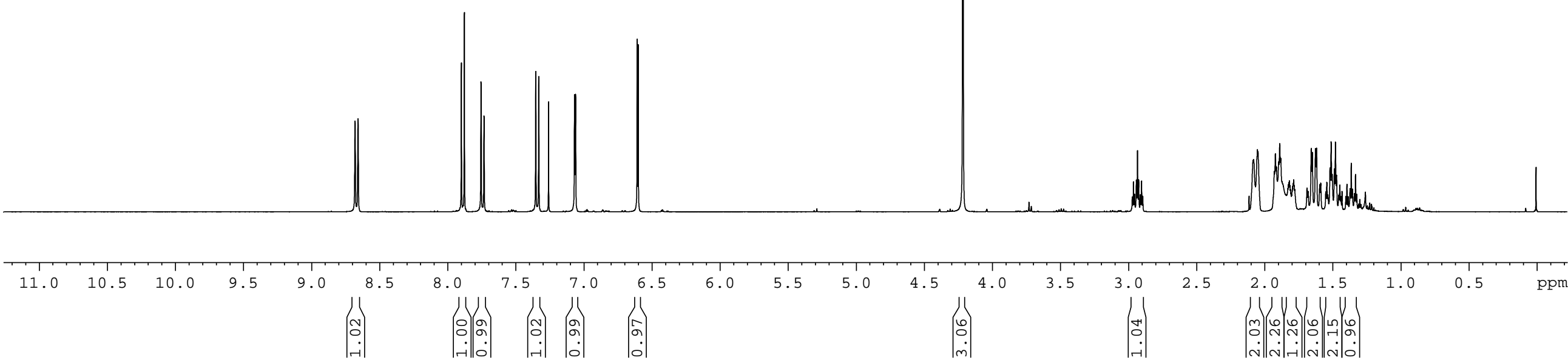




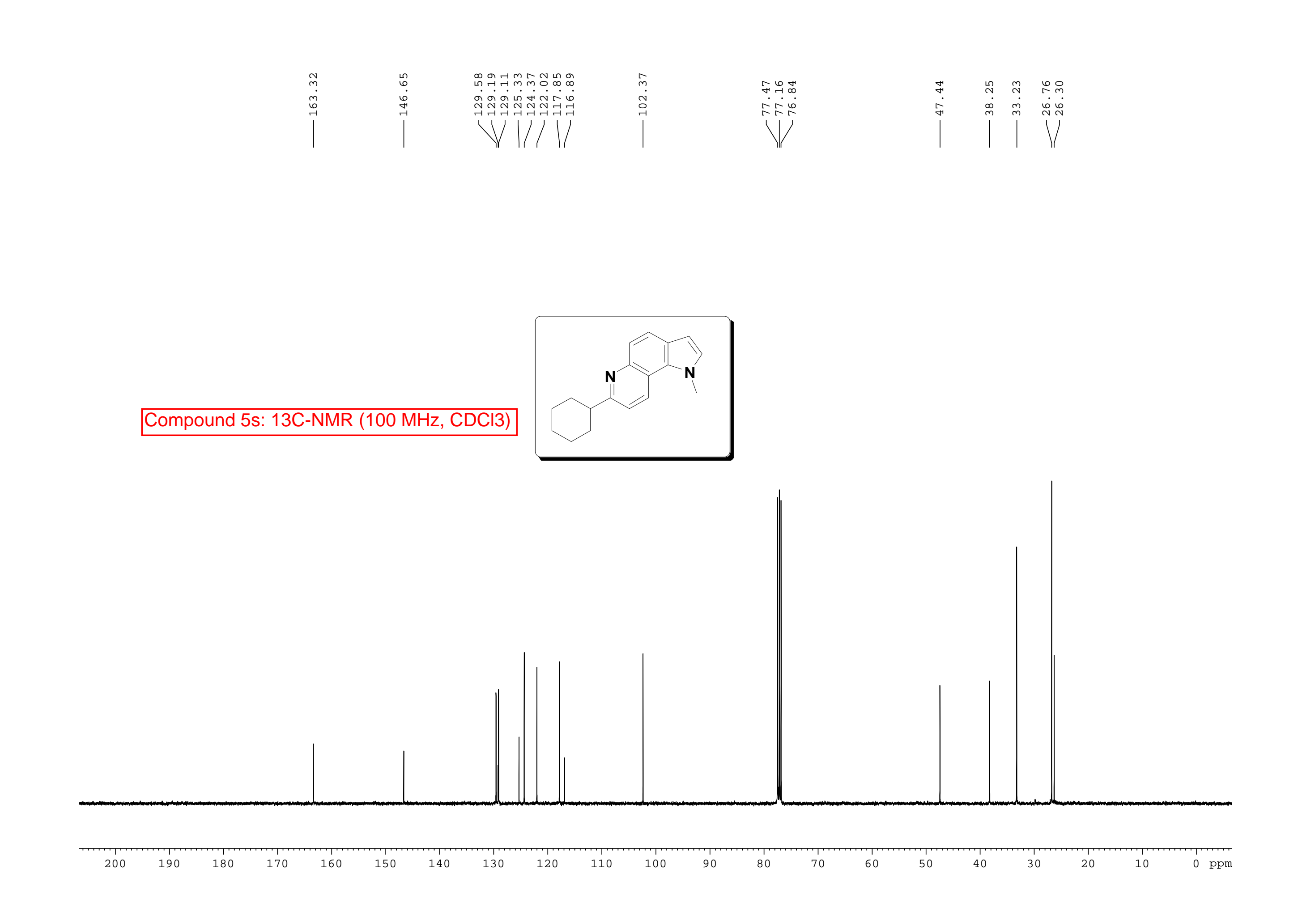




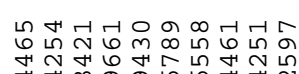

isiobicisid

V/VYVI

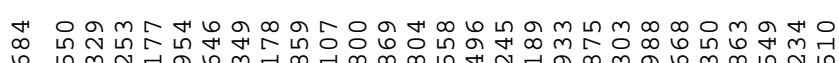

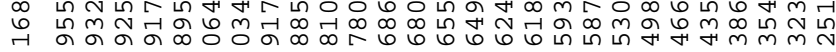

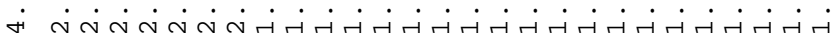
资

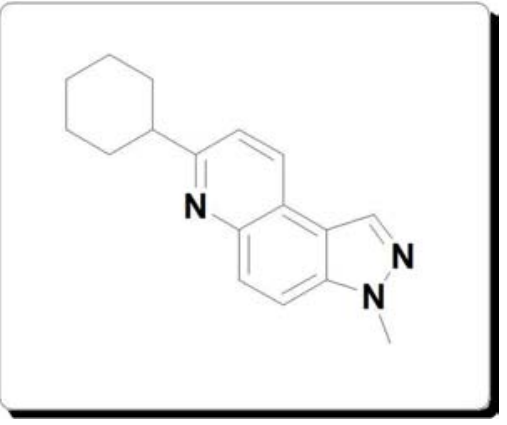

Compound 5t: 1H-NMR (400 MHz, CDCl3)
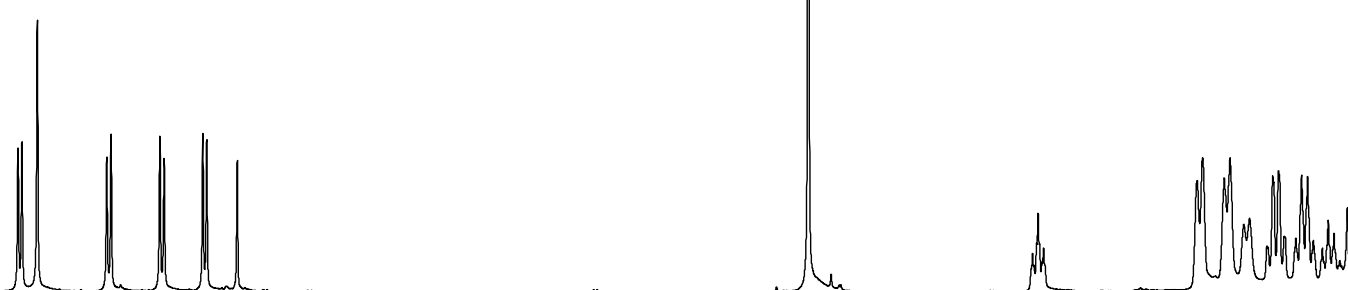

6

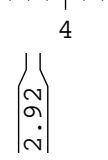




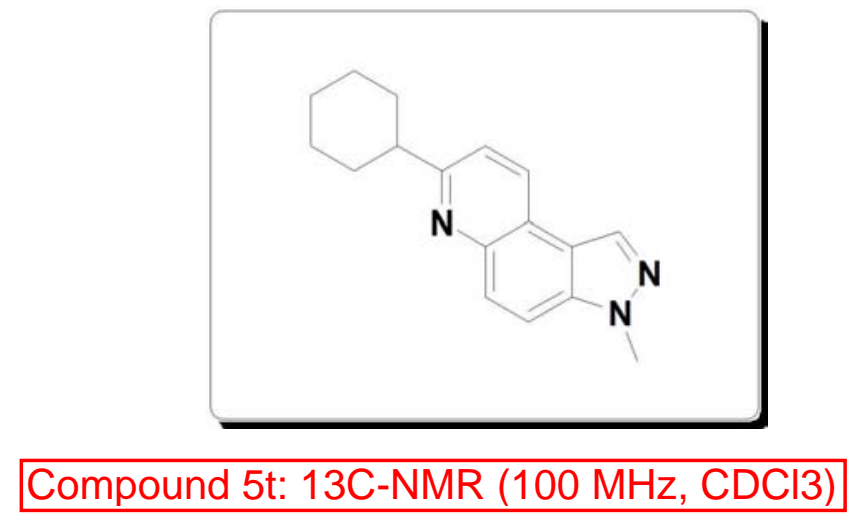

Compound 5t: 13C-NMR (100 MHz, CDCl3) 


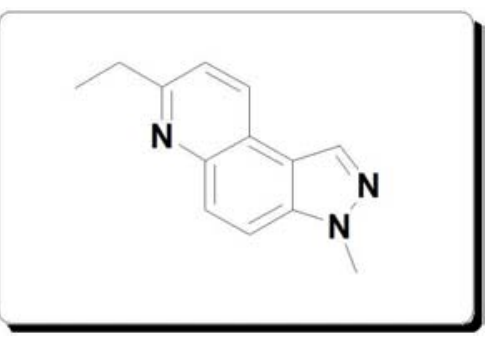

Compound 5u: 1H-NMR (400 MHz, CDCl3) 


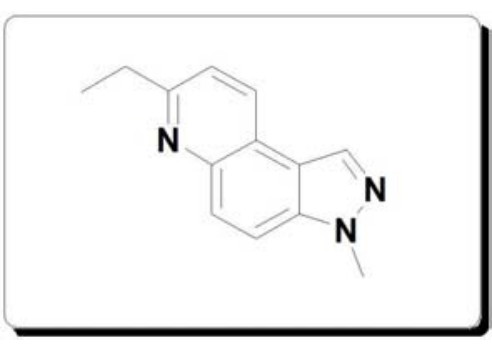

Compound 5u: 13C-NMR (100 MHz, CDCl3)
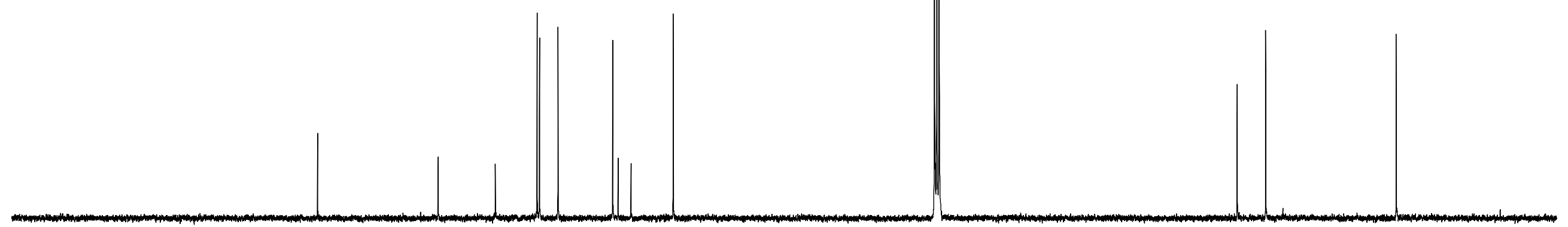

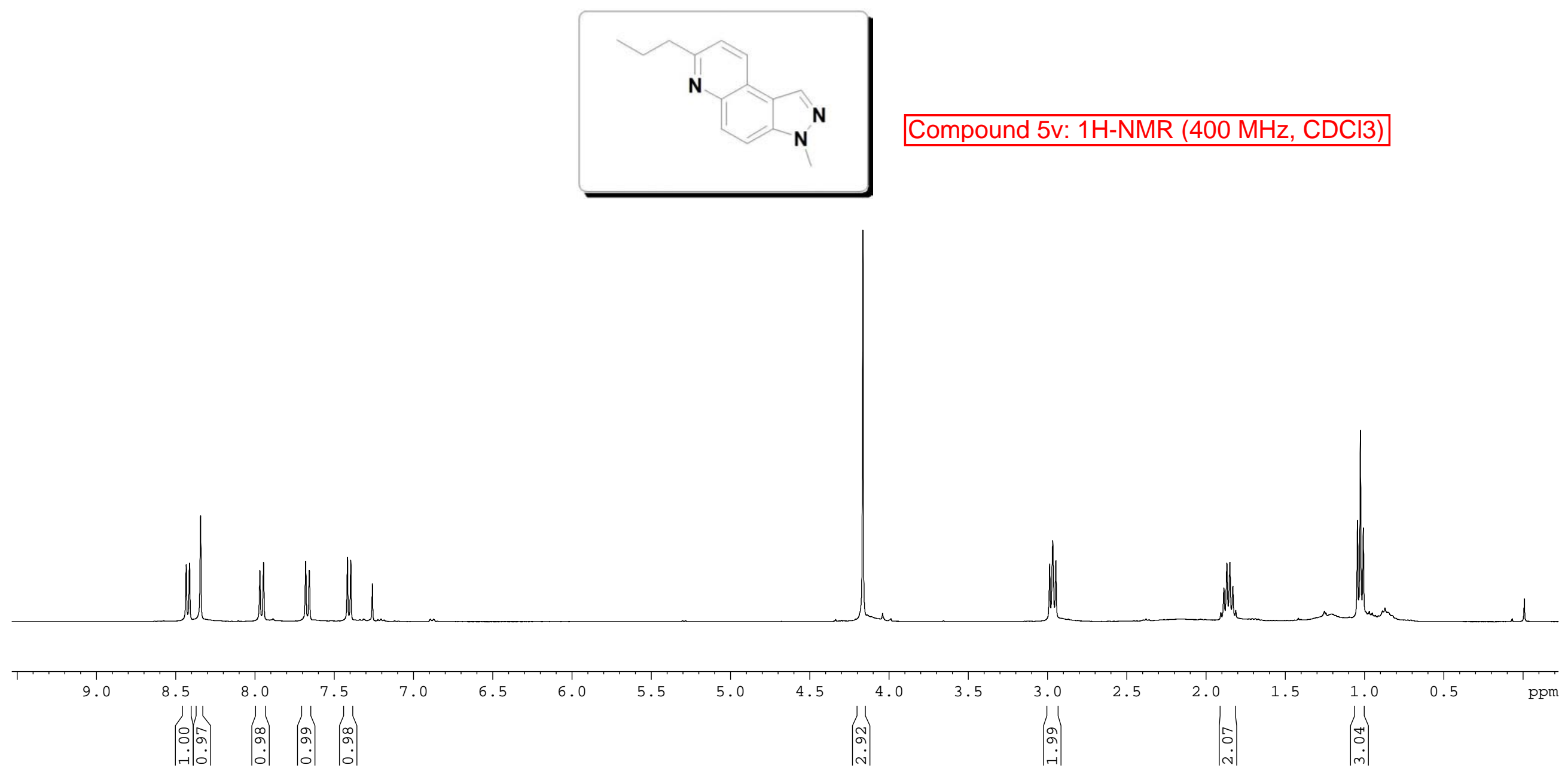


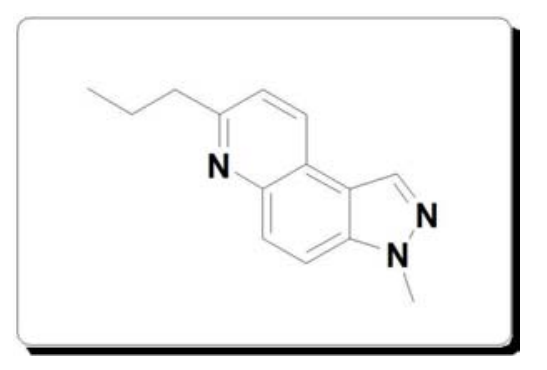

Compound 5v: 13C-NMR (100 MHz, CDCl3)

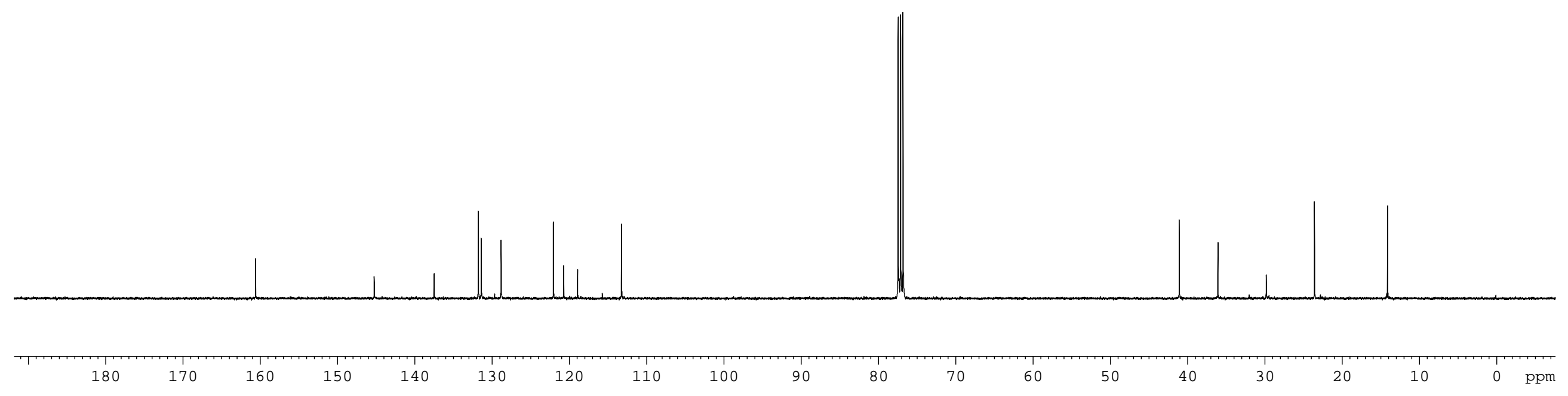




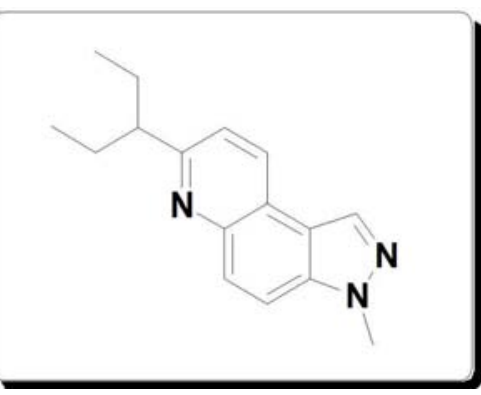

Compound 5w: 1H-NMR (400 MHz, CDCl3)

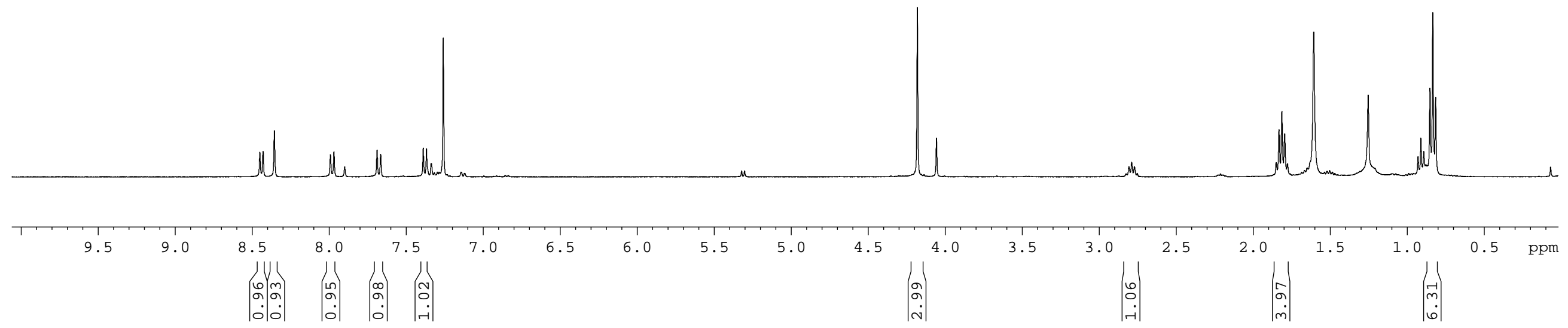




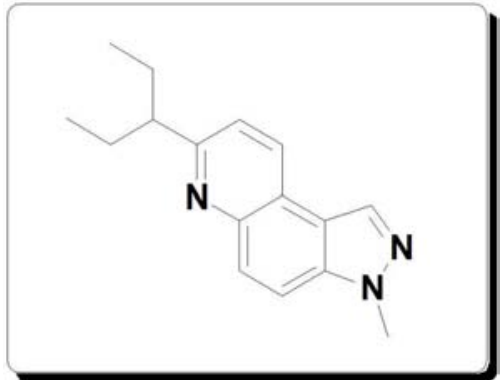

Compound 5w: 13C-NMR (100 MHz, CDCl3) 
Current Data Parameters

20151208

EXPNO 10
1

F2 - Acquisition Parameters

$\begin{array}{lr}\text { Date_- } & 20151208 \\ \text { Time- } & 17.28\end{array}$

INSTRUM

PROBHD $5 \mathrm{~mm}$ PABBO BB-

\begin{tabular}{lr} 
PULPROG & $\mathrm{zg} 30$ \\
TD & 65536 \\
\hline
\end{tabular}

NS

DS

FIDRES

AQ

AQ

DW

TE

D1

TD 0

$\mathrm{CDCl} 13$
16

$8223.685 \mathrm{~Hz}$

$0.125483 \mathrm{~Hz}$

$846387 \mathrm{sec}$

144.49
60.800 usec 6.50 usec
$298.5 \mathrm{~K}$

$1.00000000 \mathrm{sec}$

$\mathrm{Sec}$
1

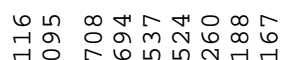

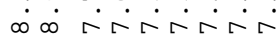

VVVIV
क्ष

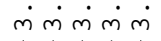

W

Compound 5x: 1H-NMR (400 MHz, CDCl3)
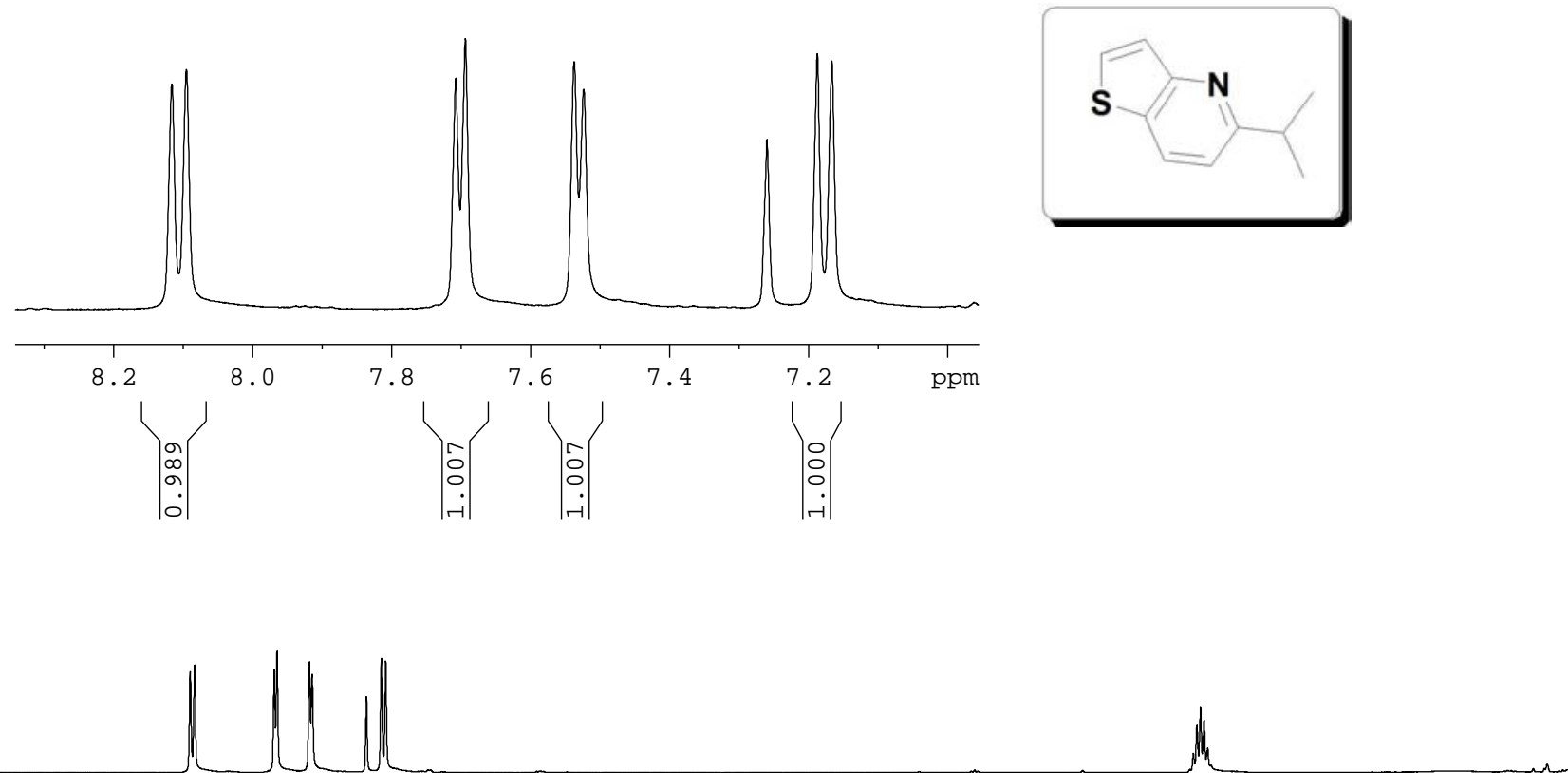

$\begin{array}{llll}11.0 & 10.5 & 10.0 & 9.5\end{array}$
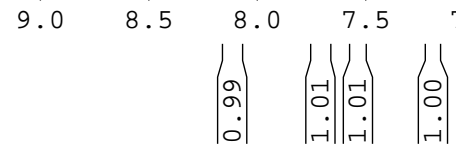

6.5

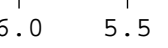

4.0

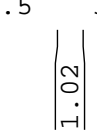

2.5
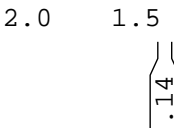


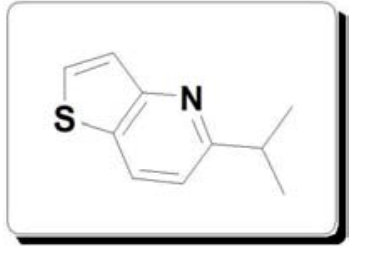

Compound 5x: 13C-NMR (100 MHz, CDCl3)

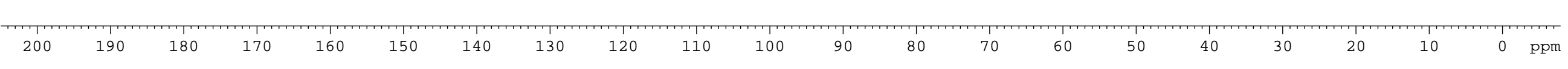




\section{HRMS (ESI)}

\section{1-benzyl-5-isopropyl-1H-pyrazolo[4,3-b]pyridine}

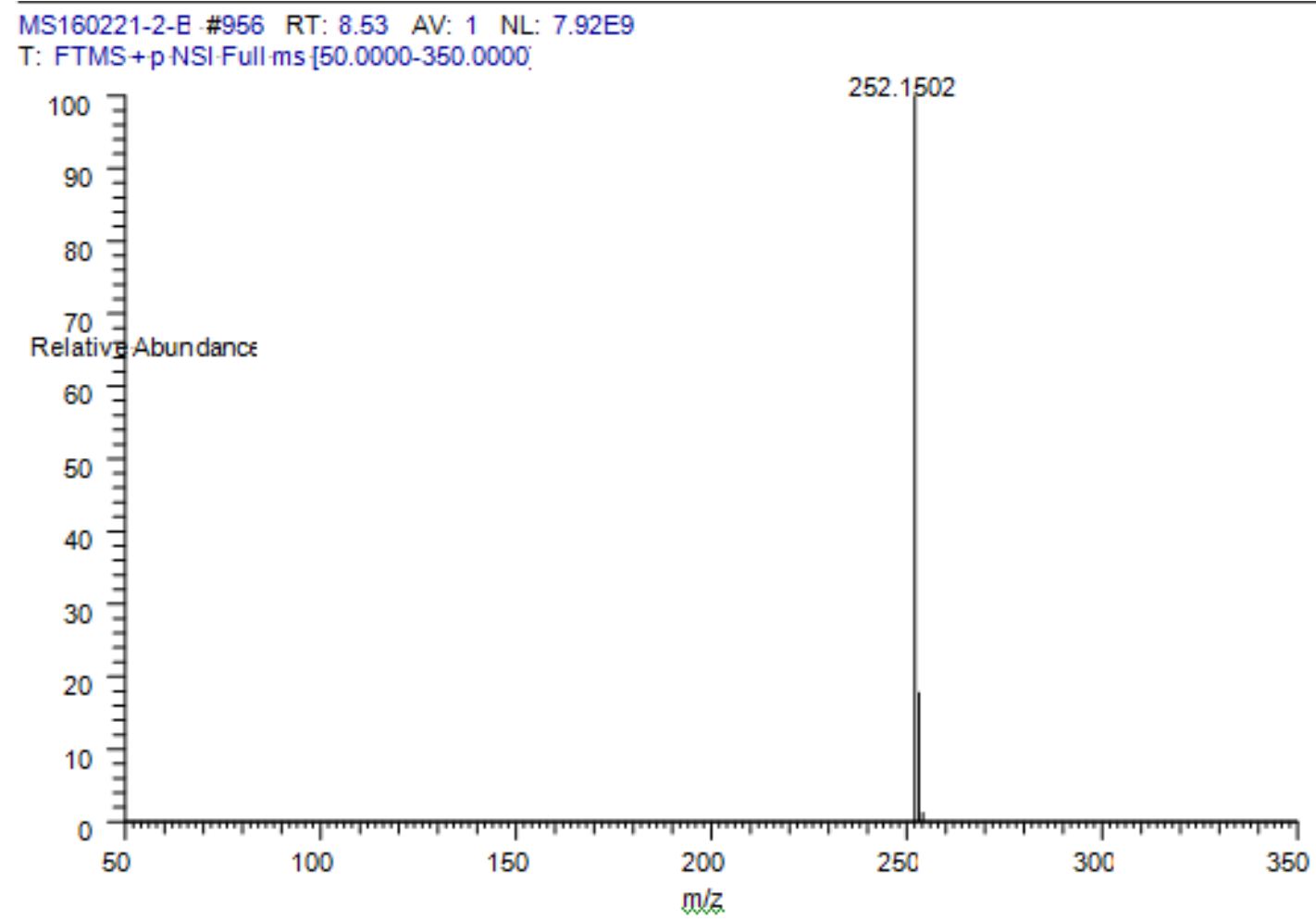

4a. 1-(5-isopropylfuro[3,2-b]pyridin-2-yl)ethanone

MS160221-2-E \#633 RT: 6.07 AV: 1 NL: 9.12E3

T: FTMS + p NSI Full ms [50.0000-350.0000]

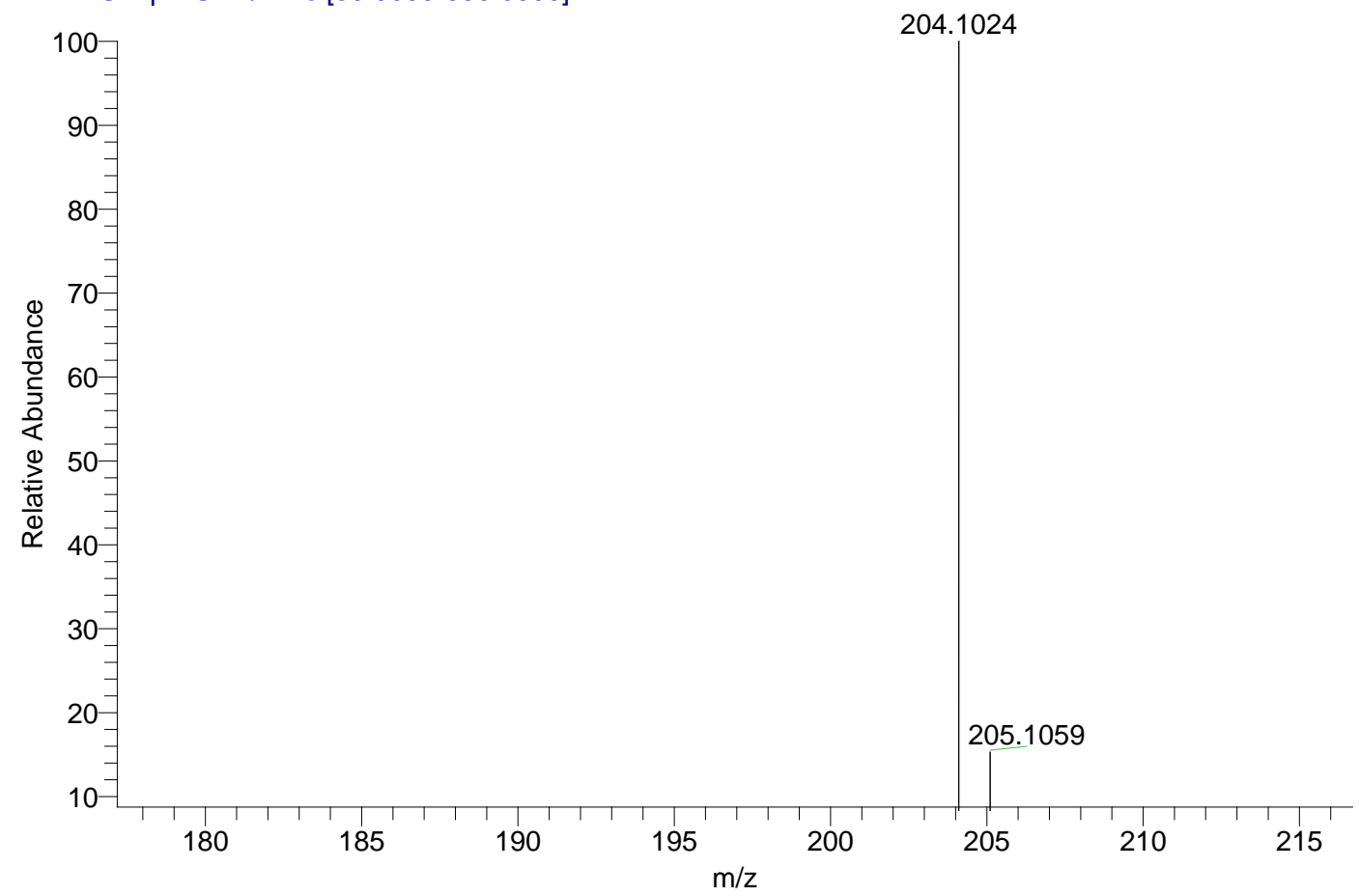


4b. 5-isopropyl-1-methyl-1H-pyrazolo[4,3-b]pyridine

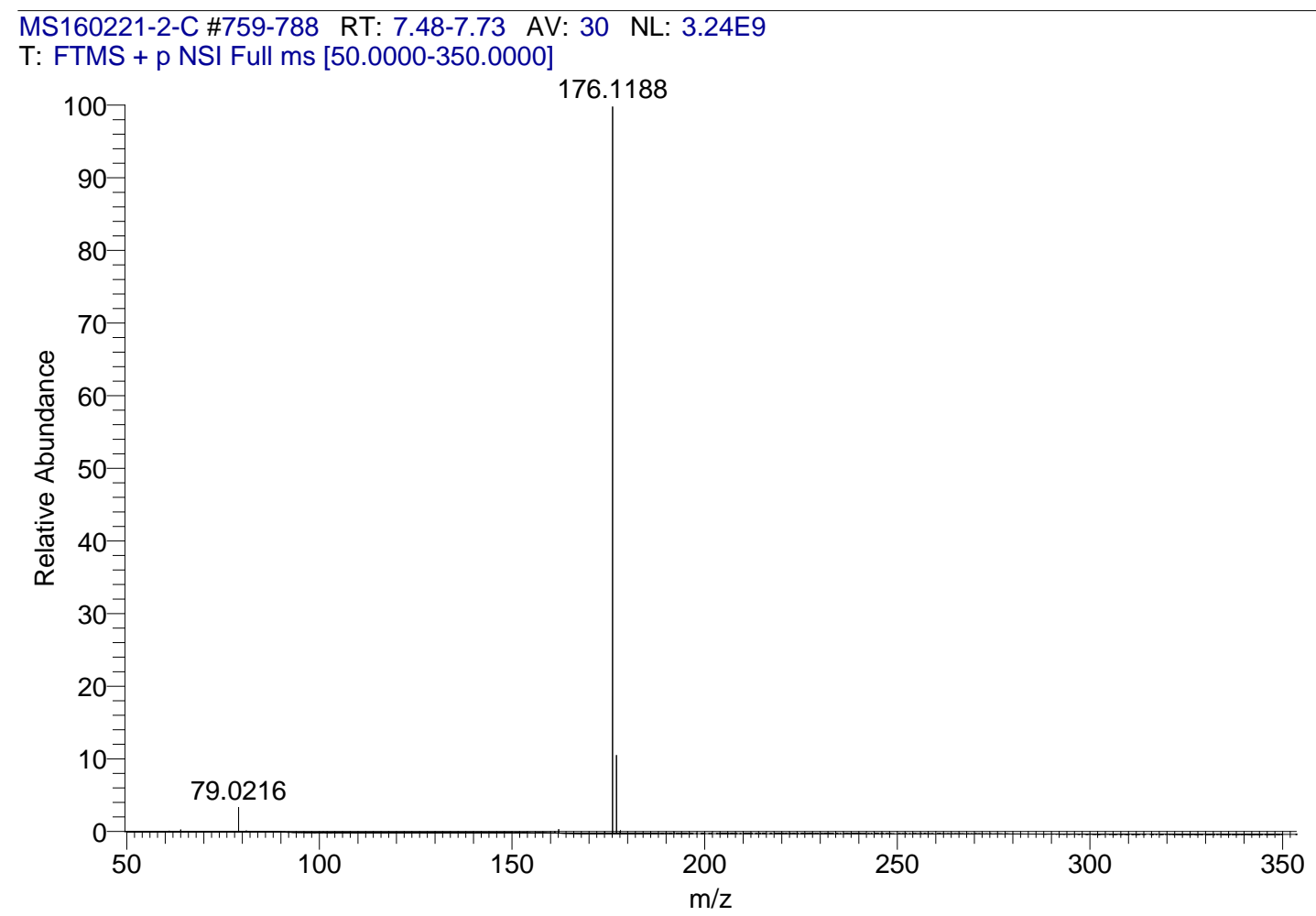

4c. 1-(5-isopropyl-1-methyl-1H-pyrrolo[3,2-b]pyridin-2-yl)ethanone

MS160221-12-H \#970 RT: 8.71 AV: 1 NL: 8.11E9

T: FTMS + p NSI Full ms [50.0000-350.0000]

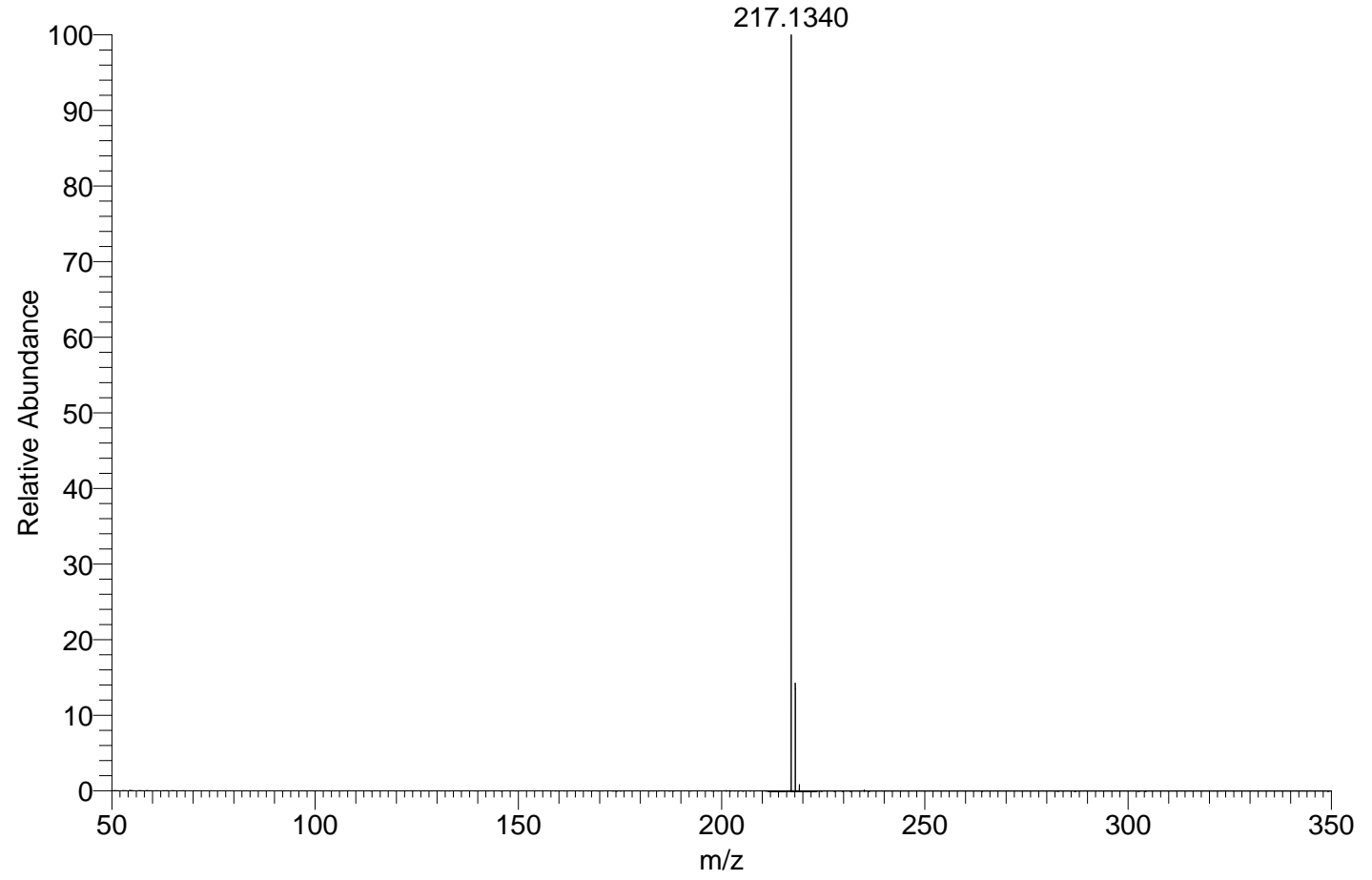


4d. 1-(2-isopropyl-6-methyl-6H-pyrrolo[3,4-b]pyridin-7-yl)ethanone

MS160221-2-D \#1196-1288 RT: 11.17-12.00 AV: 93 NL: 4.95E9

T: FTMS + p NSI Full ms [50.0000-350.0000]

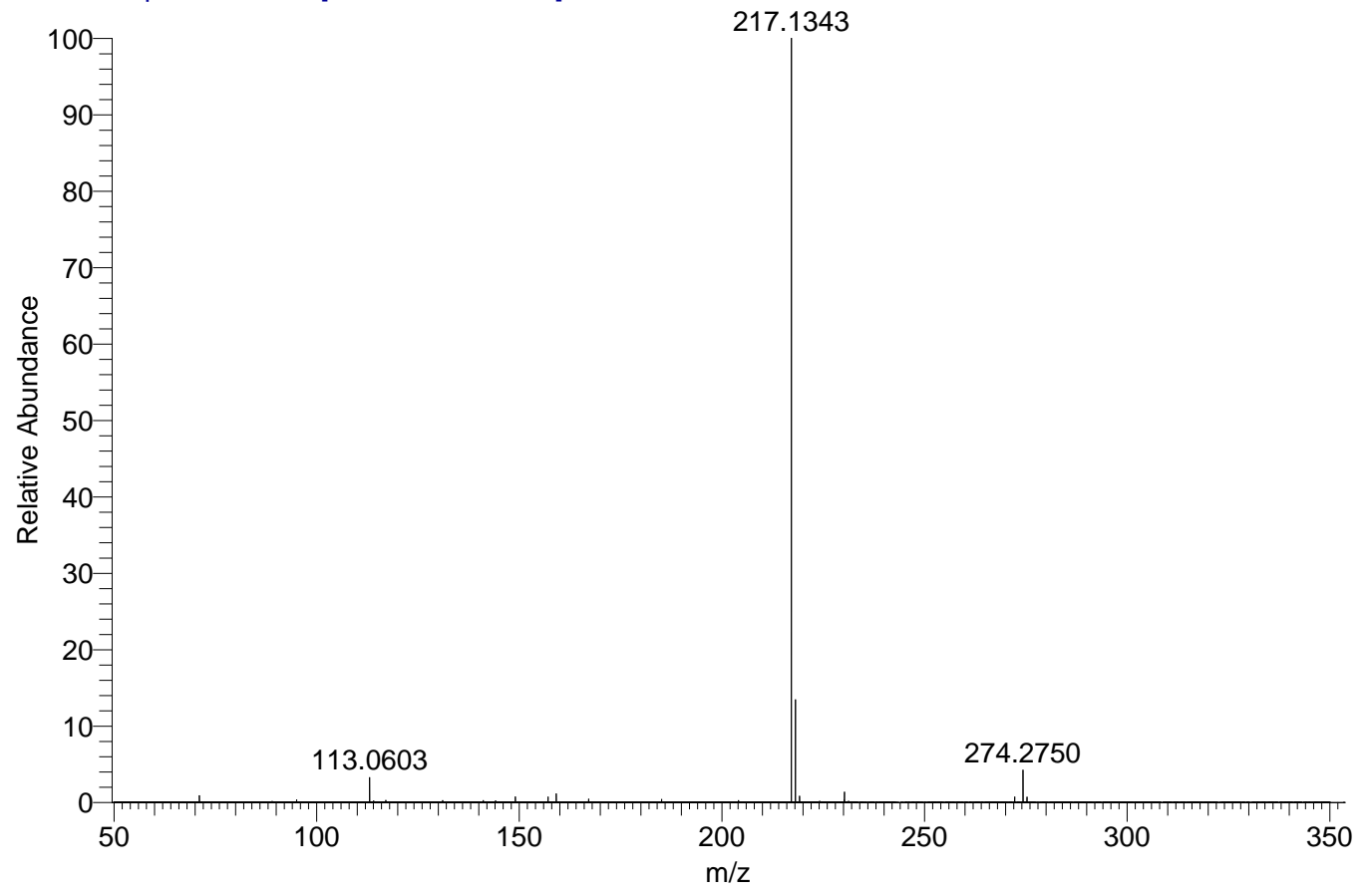

4e. 2-isopropylthieno[3,4-b]pyridine

MS160221-1-A \#662 RT: 6.23 AV: 1 NL: 3.60E6

T: FTMS + p NSI Full ms [50.0000-350.0000]

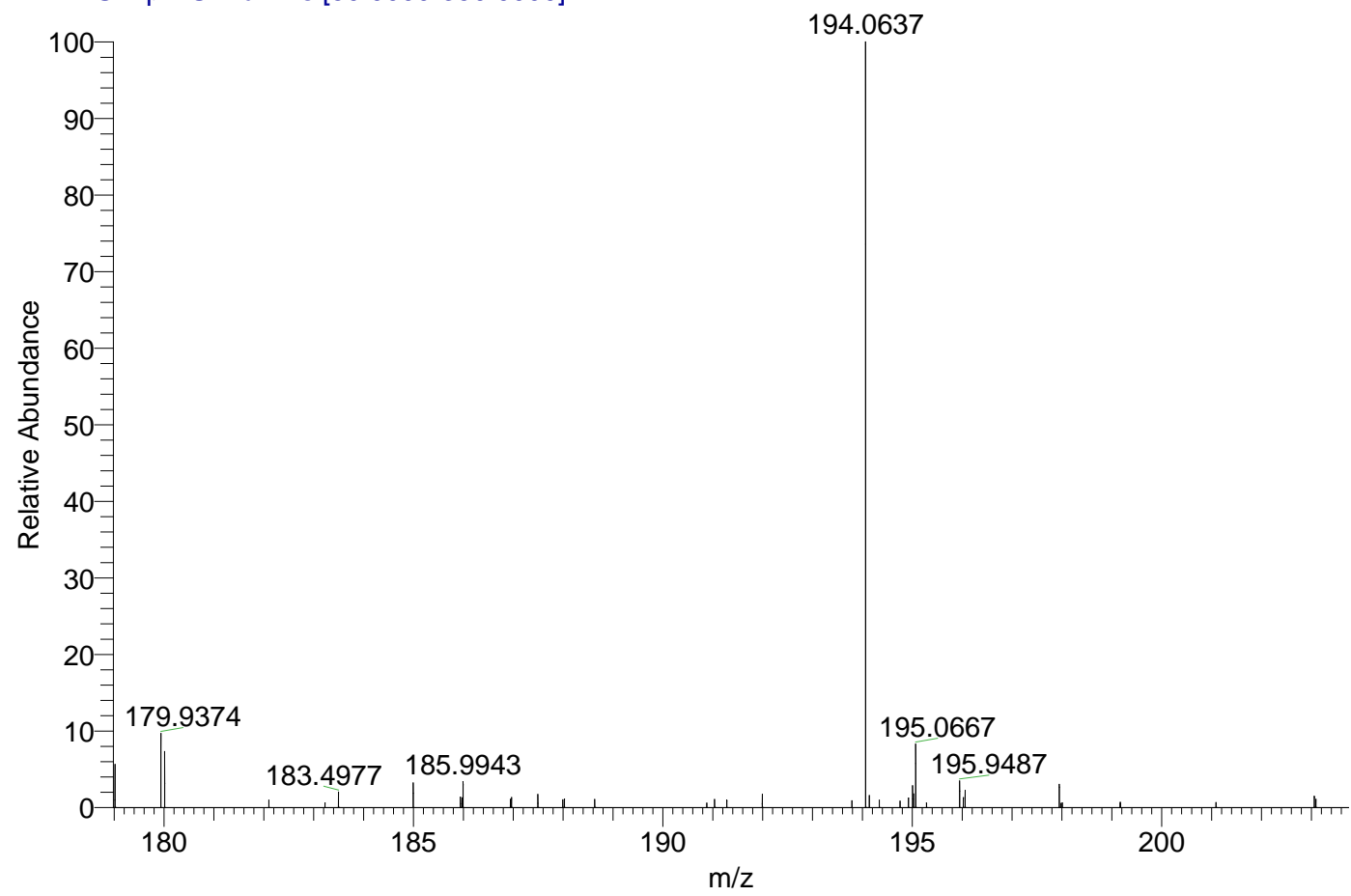


4f. 7-isopropyl-1-methyl-1H-pyrrolo[2,3-f]quinolone

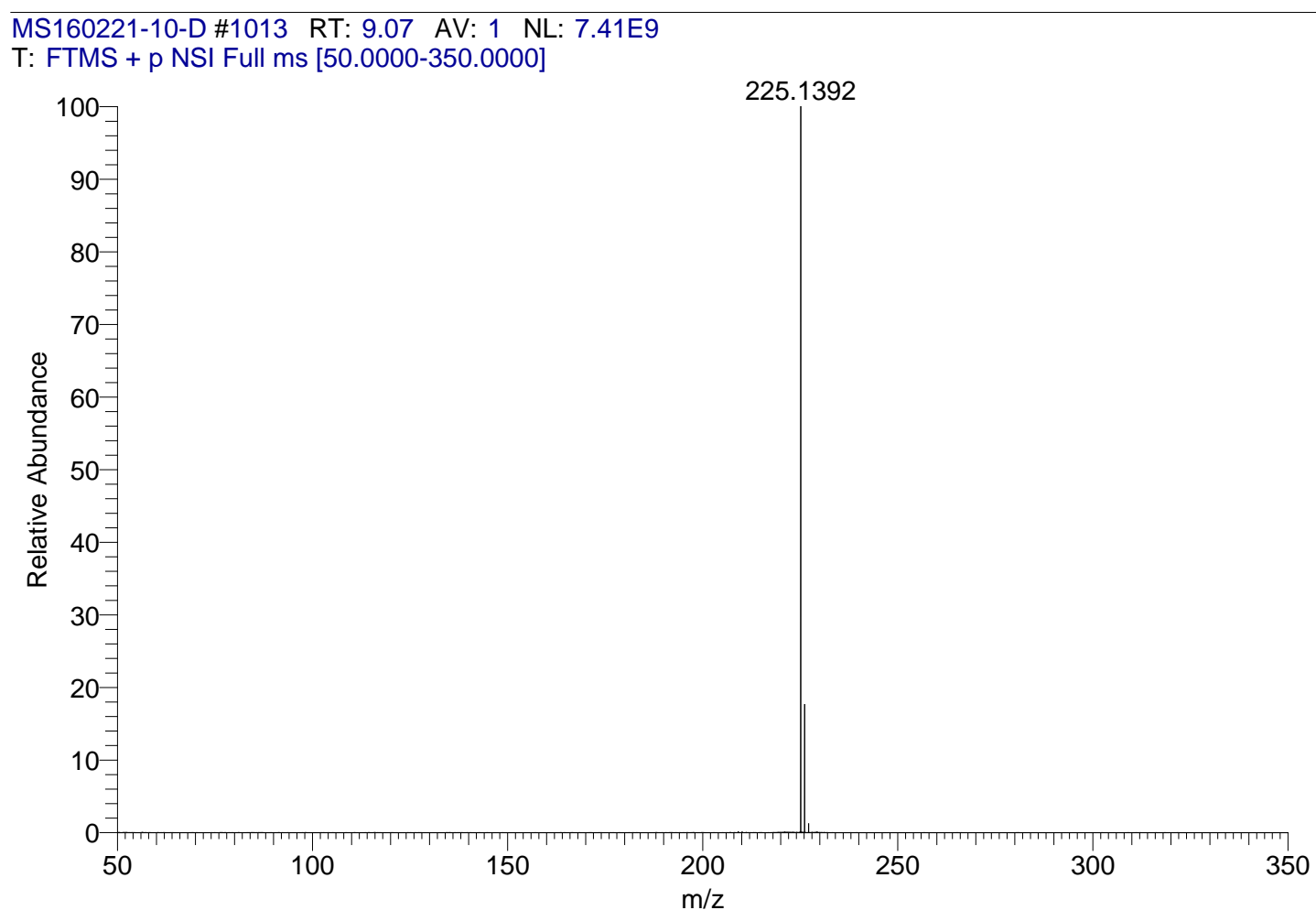

4g. 7-isopropyl-3-methyl-3H-pyrrolo[3,2-f]quinolone

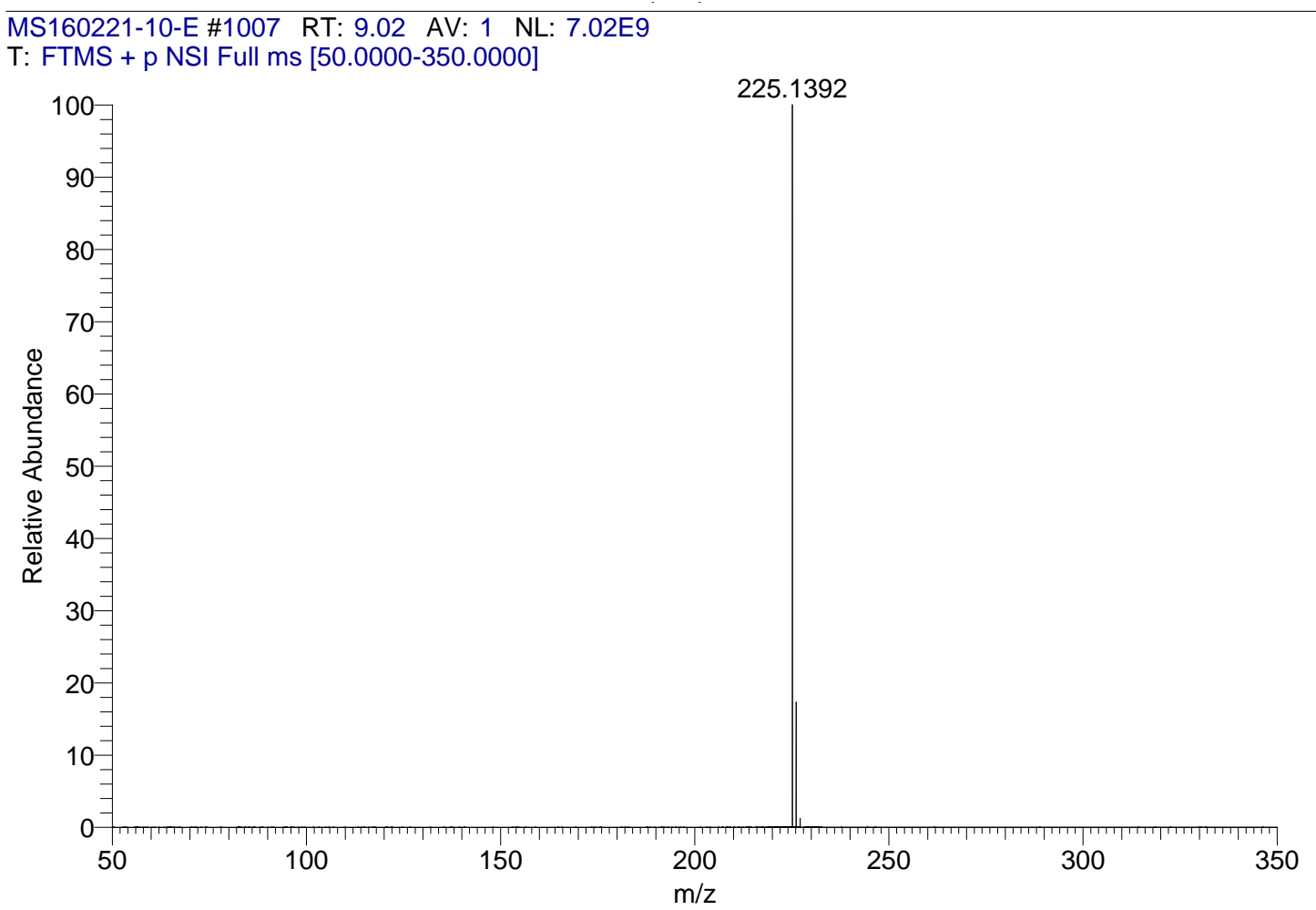


4h. 8-isopropyl-1-methyl-1H-pyrrolo[3,2-h]quinolone

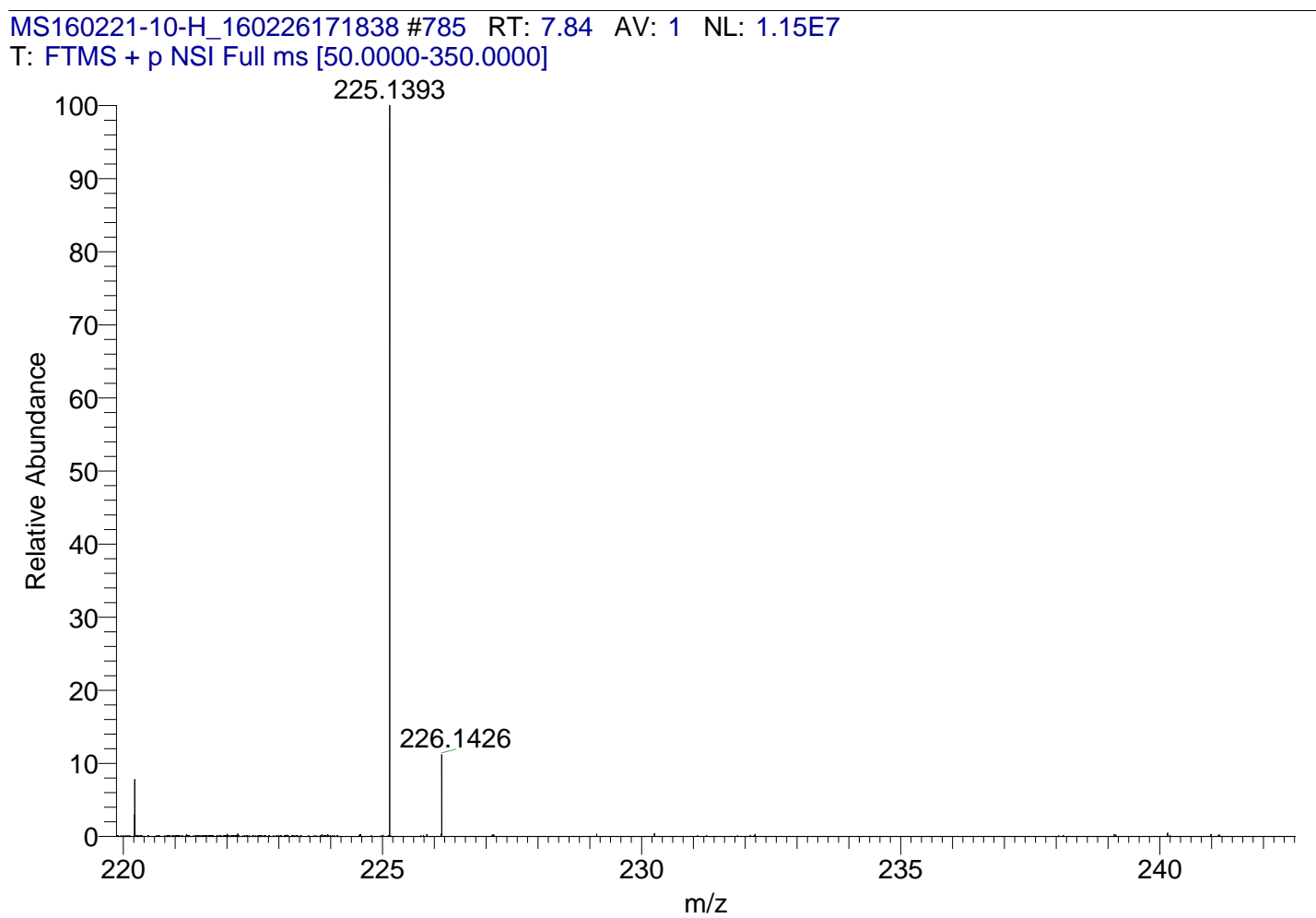

4i. 7-isopropyl-3-phenyl-3H-pyrrolo[3,2-f]quinolone

MS160221-11-F \#1220 RT: 10.93 AV: 1 NL: 2.50E9

T: FTMS + p NSI Full ms [50.0000-350.0000]

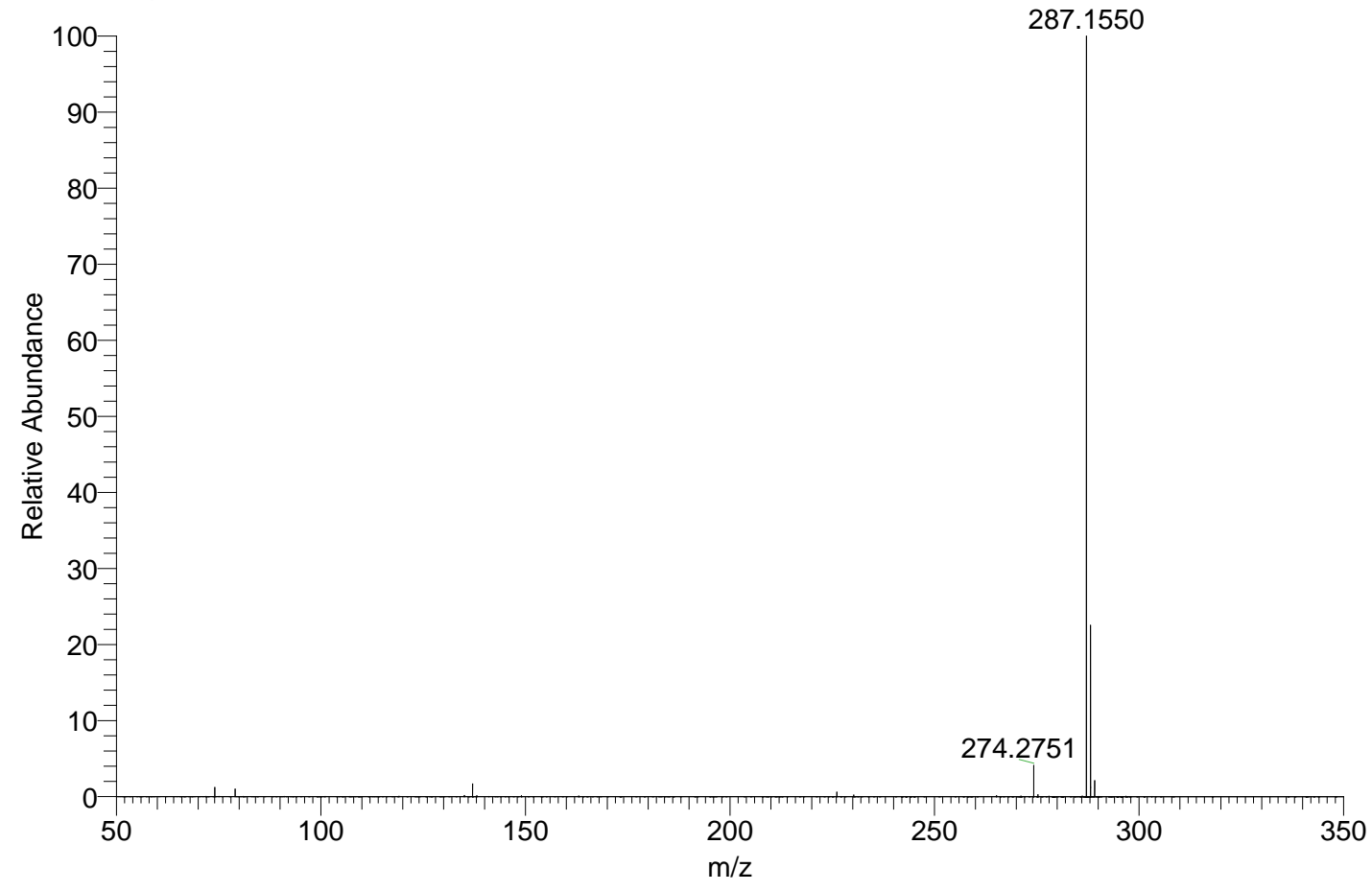


4j. 7-isopropyl-3-phenyl-3H-pyrazolo[4,3-f]quinolone

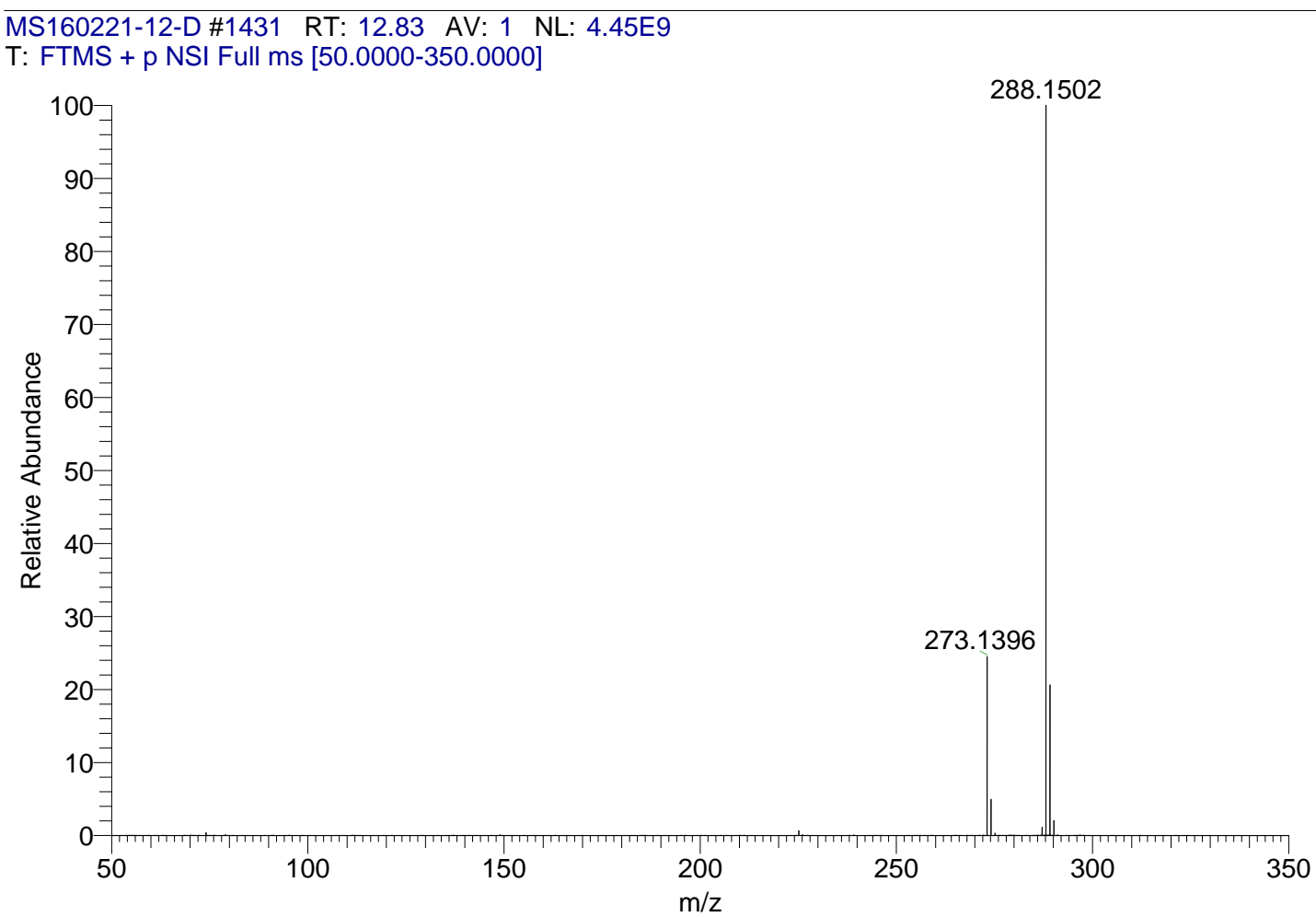

4k. 7-isopropyl-3-methyl-3H-pyrazolo[4,3-f]quinolone

MS160221-12-A \#1050-1115 RT: 9.40-9.98 AV: 66 NL: 7.47E9

T: FTMS + p NSI Full ms [50.0000-350.0000]

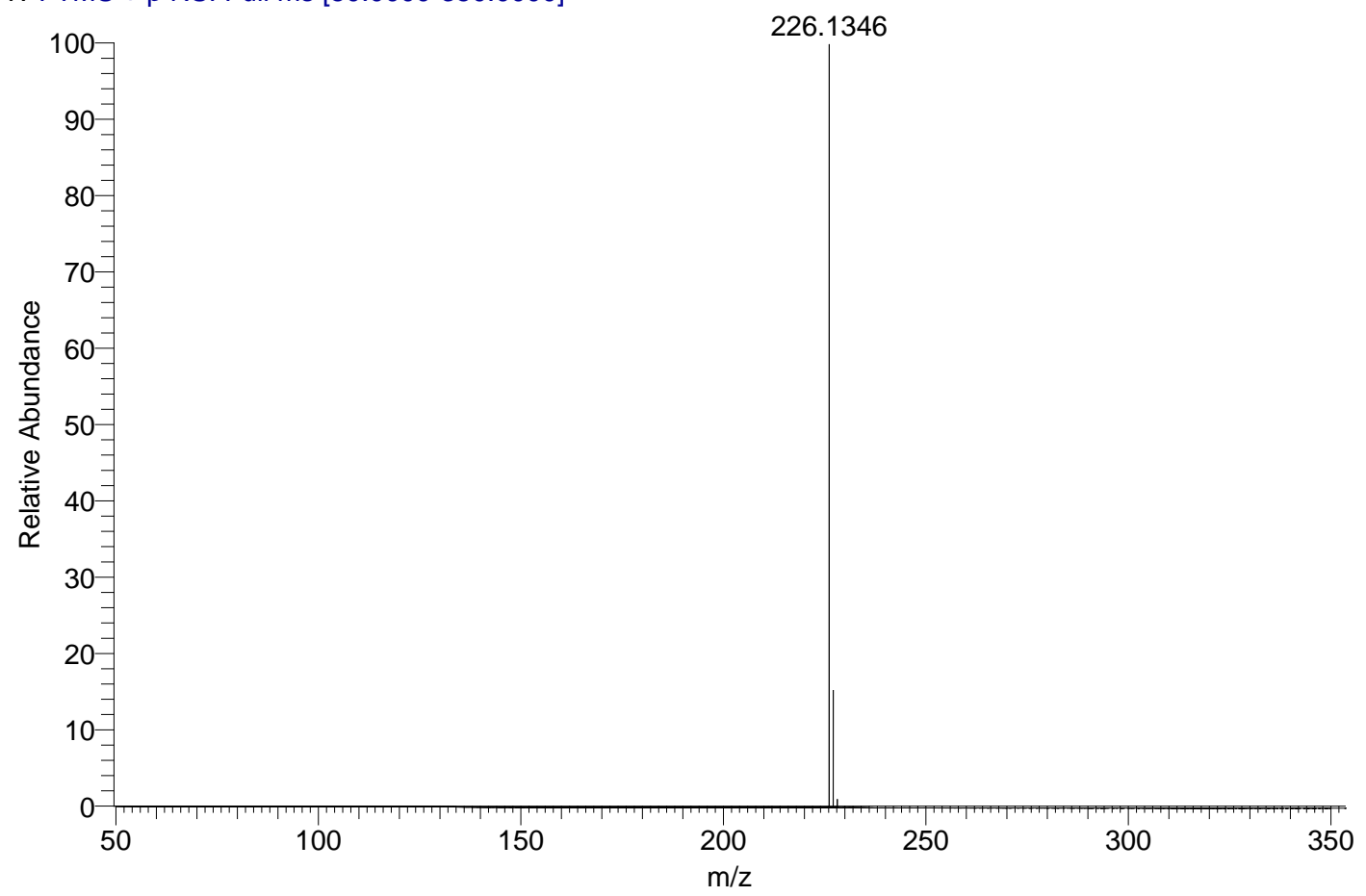


4l. 7-isopropyl-2-methyl-2H-pyrazolo[4,3-f]quinolone

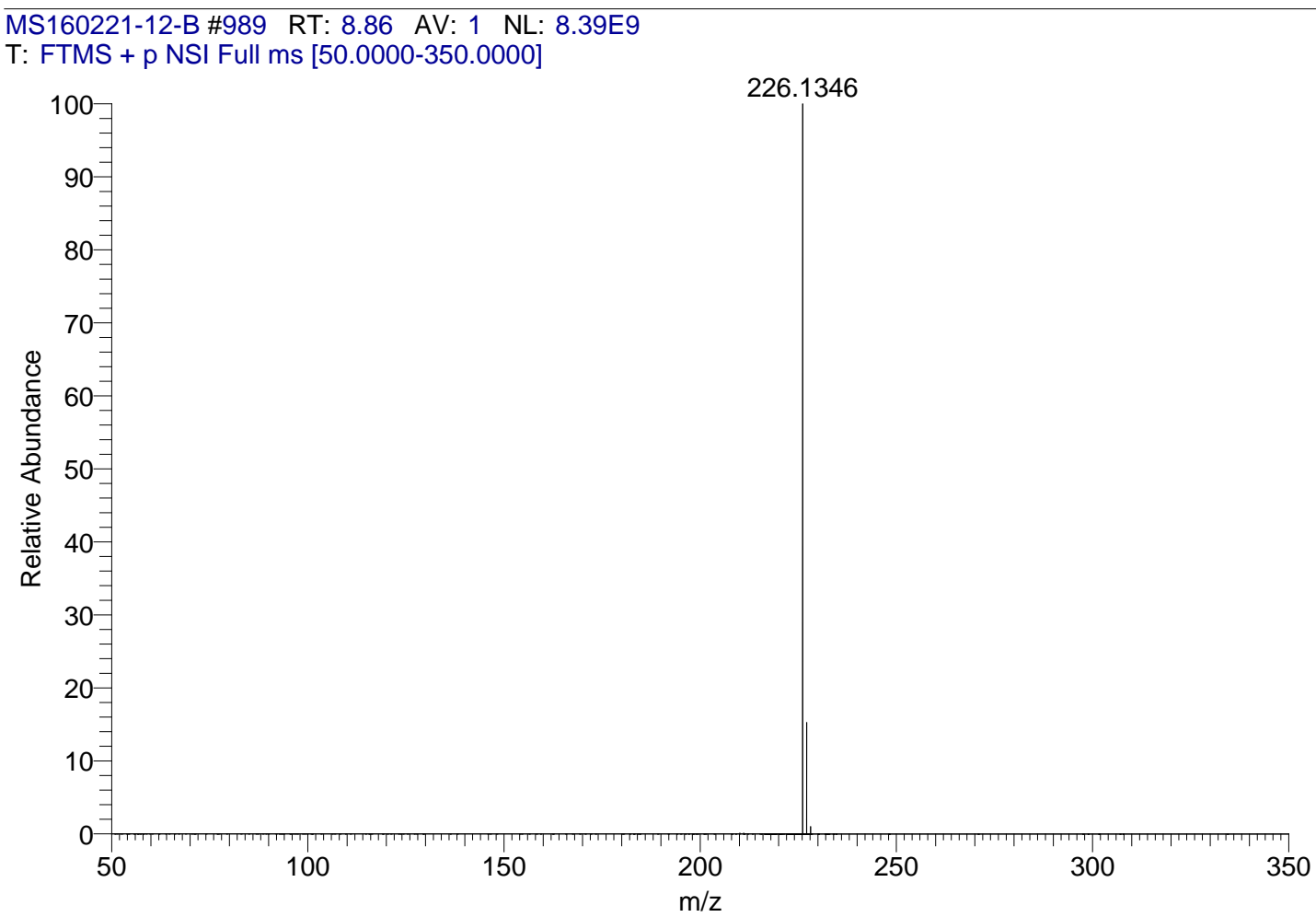

4m. 7-isopropyl-2-methyl-2H-pyrazolo[3,4-f]quinolone

MS160221-12-C \#965-1055 RT: 8.66-9.46 AV: 91 NL: 7.39E9 T: FTMS + p NSI Full ms [50.0000-350.0000]

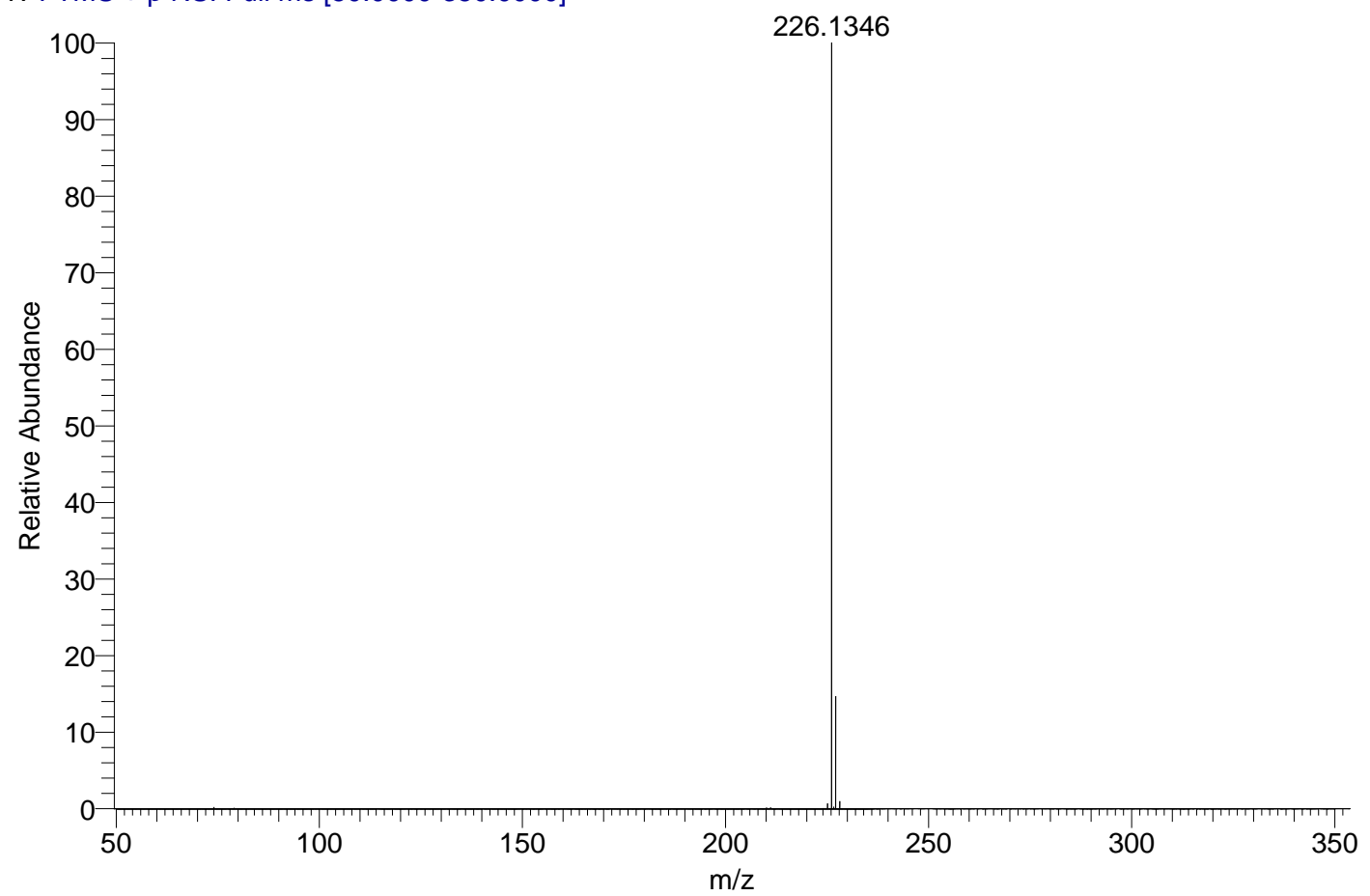


4n. 7-isopropyl-1-methyl-1H-pyrazolo[3,4-f]quinolone

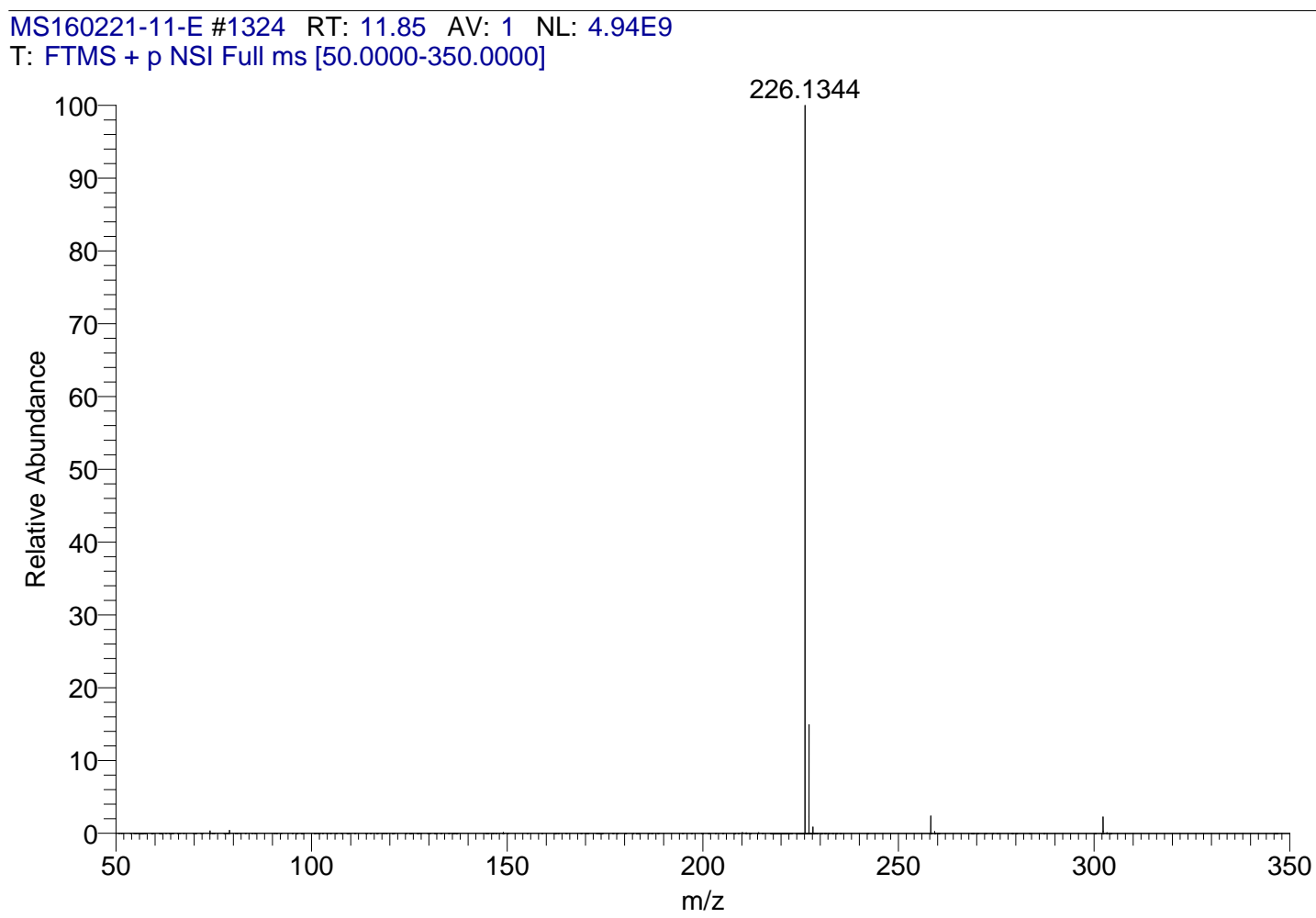

4o-1. 7-isopropyl-1-phenyl-1H-pyrazolo[3,4-f]quinolone

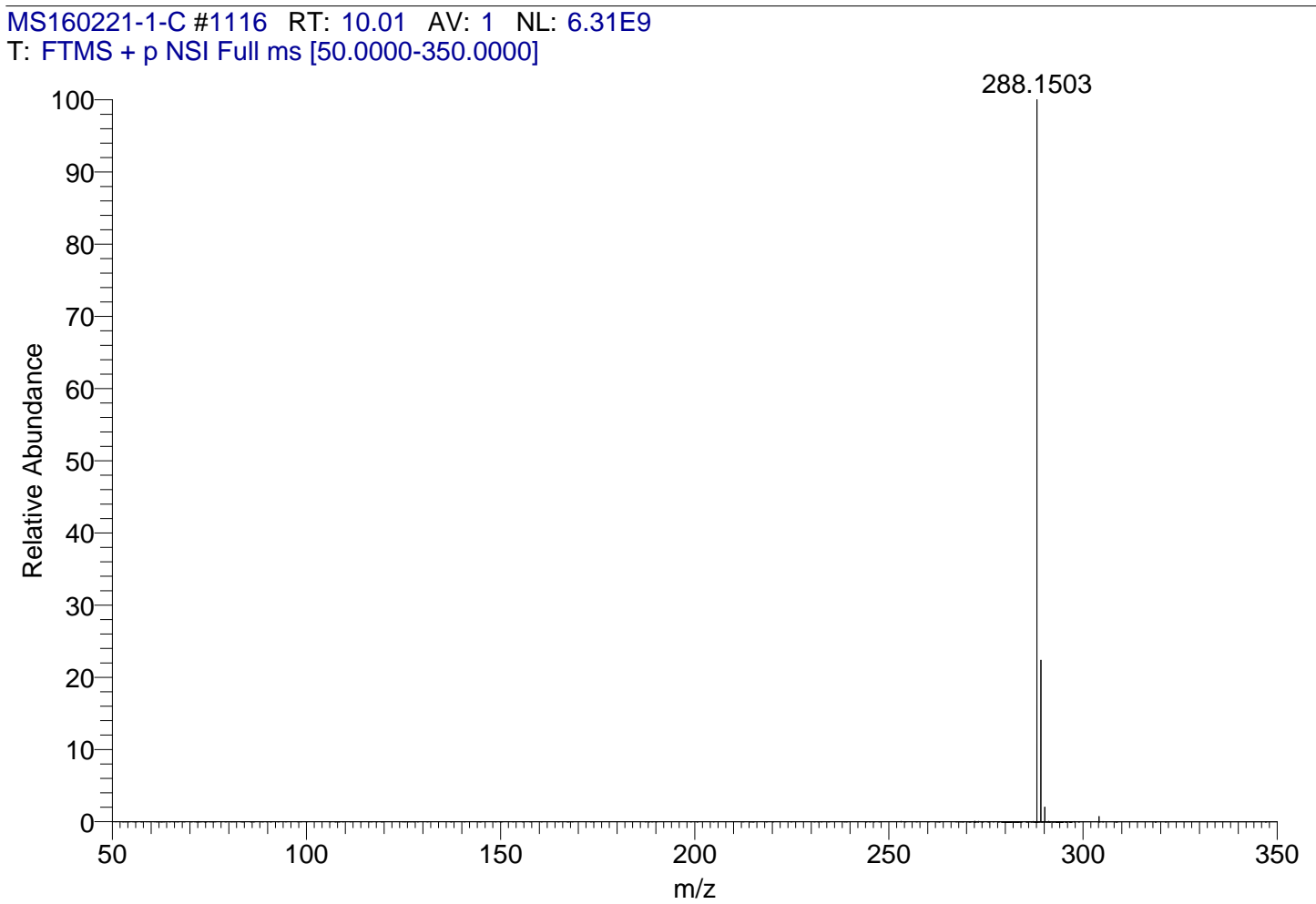


4o-2. 7-isopropyl-1-phenyl-1H-pyrazolo[4,3-g]quinolone

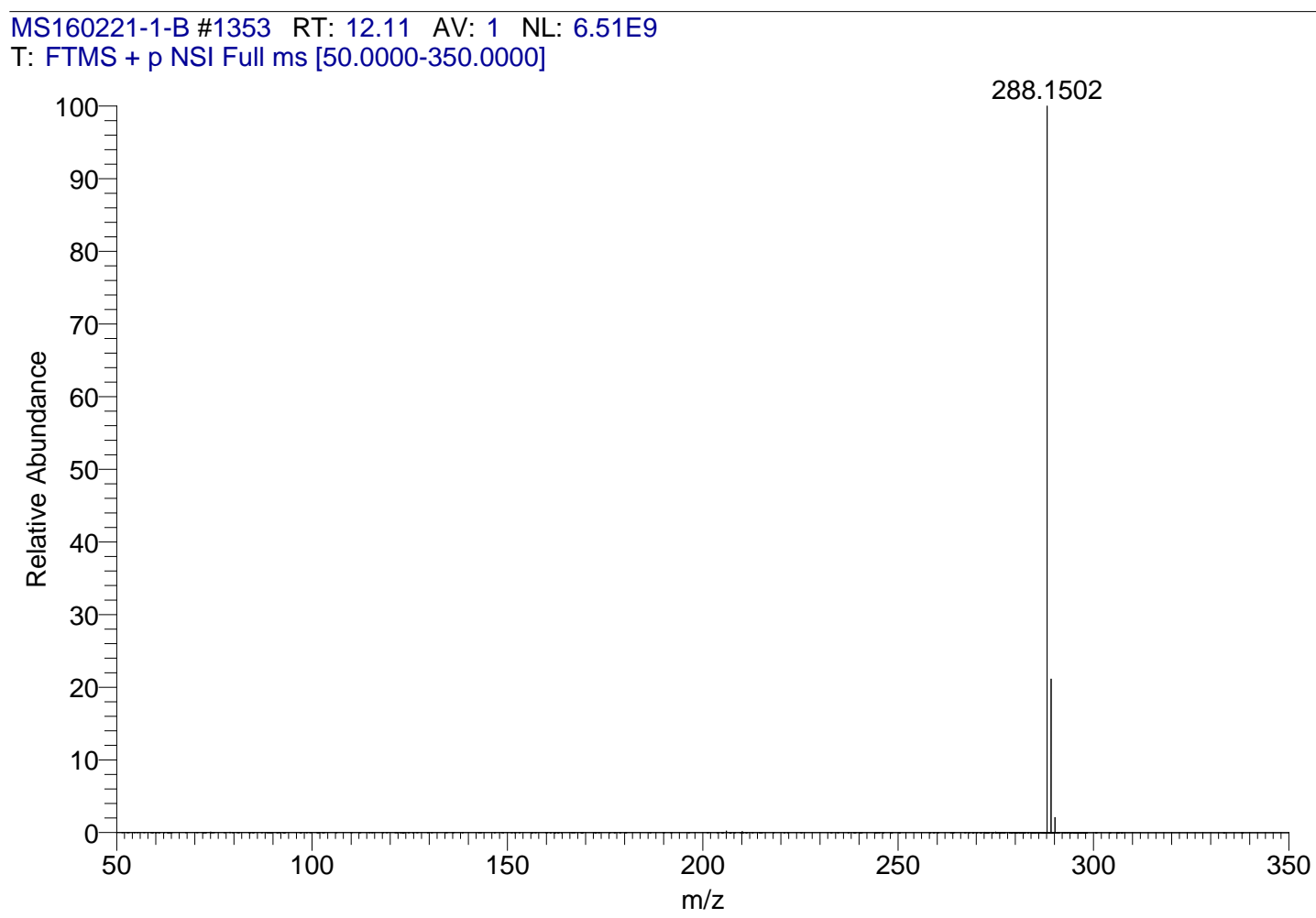

5a. 1-(5-isopropylfuro[3,2-b]pyridin-2-yl)ethanone

MS160221-3-A 160226160031 \#344 RT: 3.06 AV: 1 NL: 2.08E9 T: FTMS + p NSI Full ms [50.0000-350.0000]

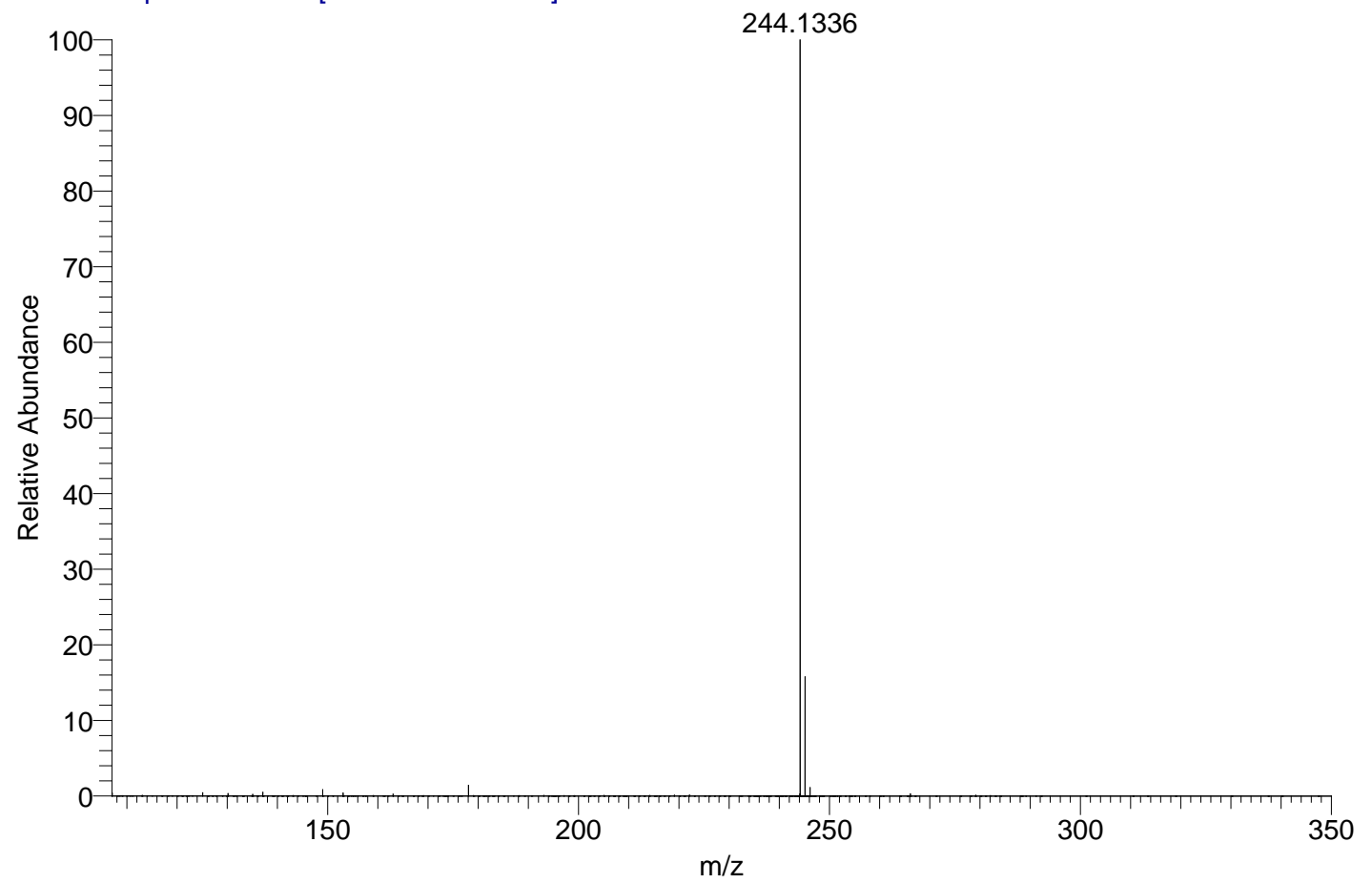


$5 b$.

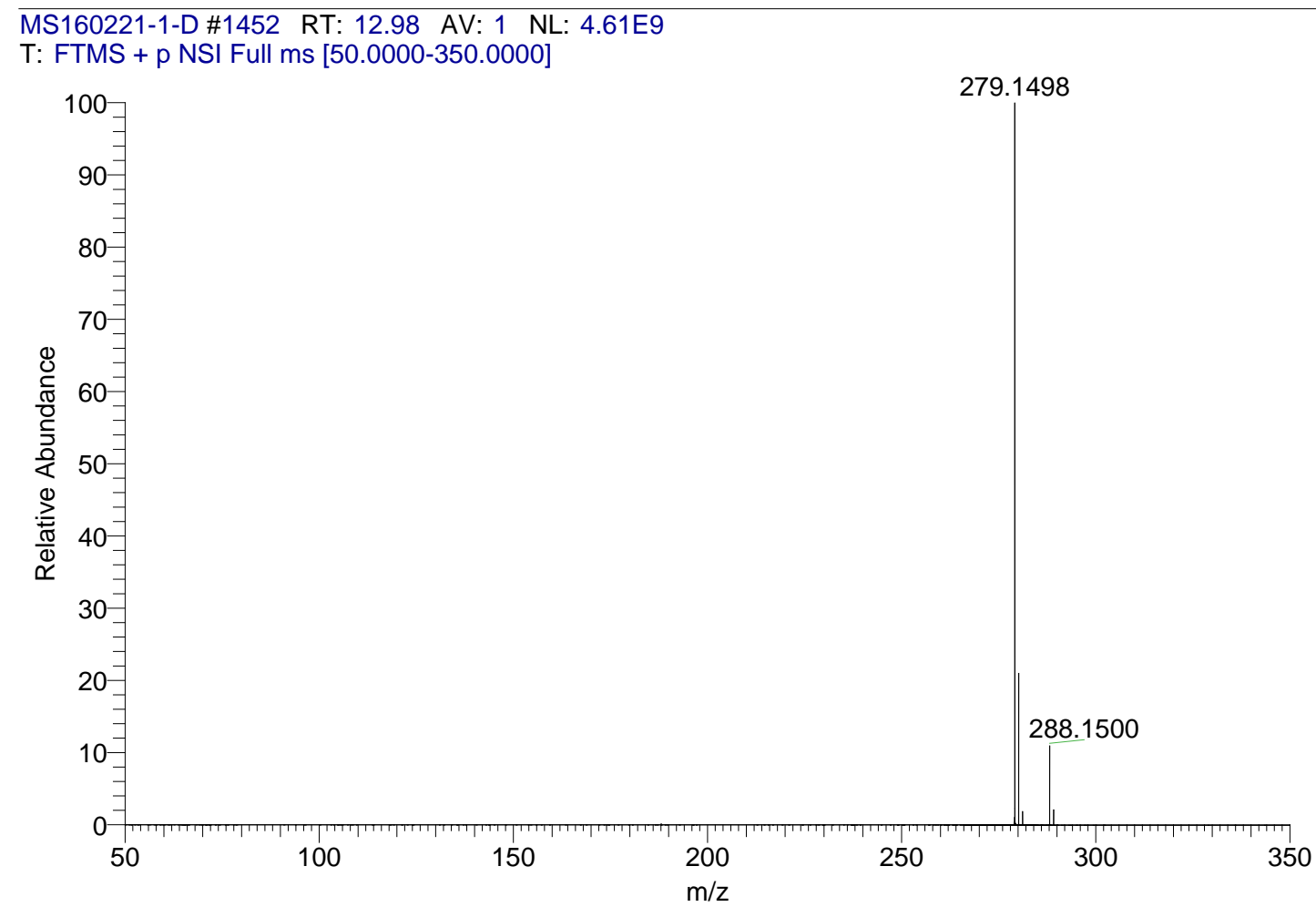

5c. 1-(5-ethyl-1-methyl-1H-pyrrolo[3,2-b]pyridin-2-yl)ethanone

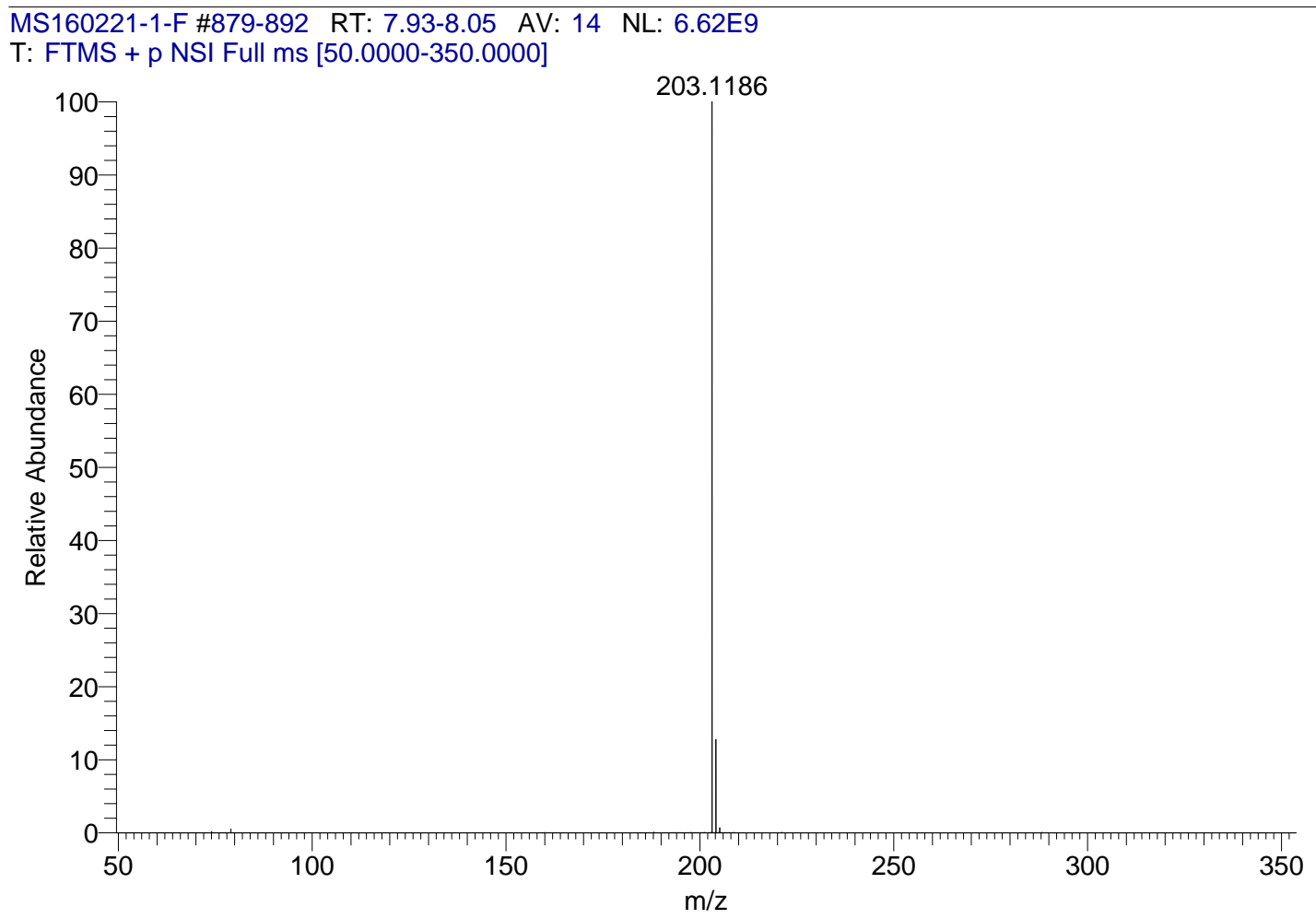


5d. 1-(1-methyl-5-propyl-1H-pyrrolo[3,2-b]pyridin-2-yl)ethanone

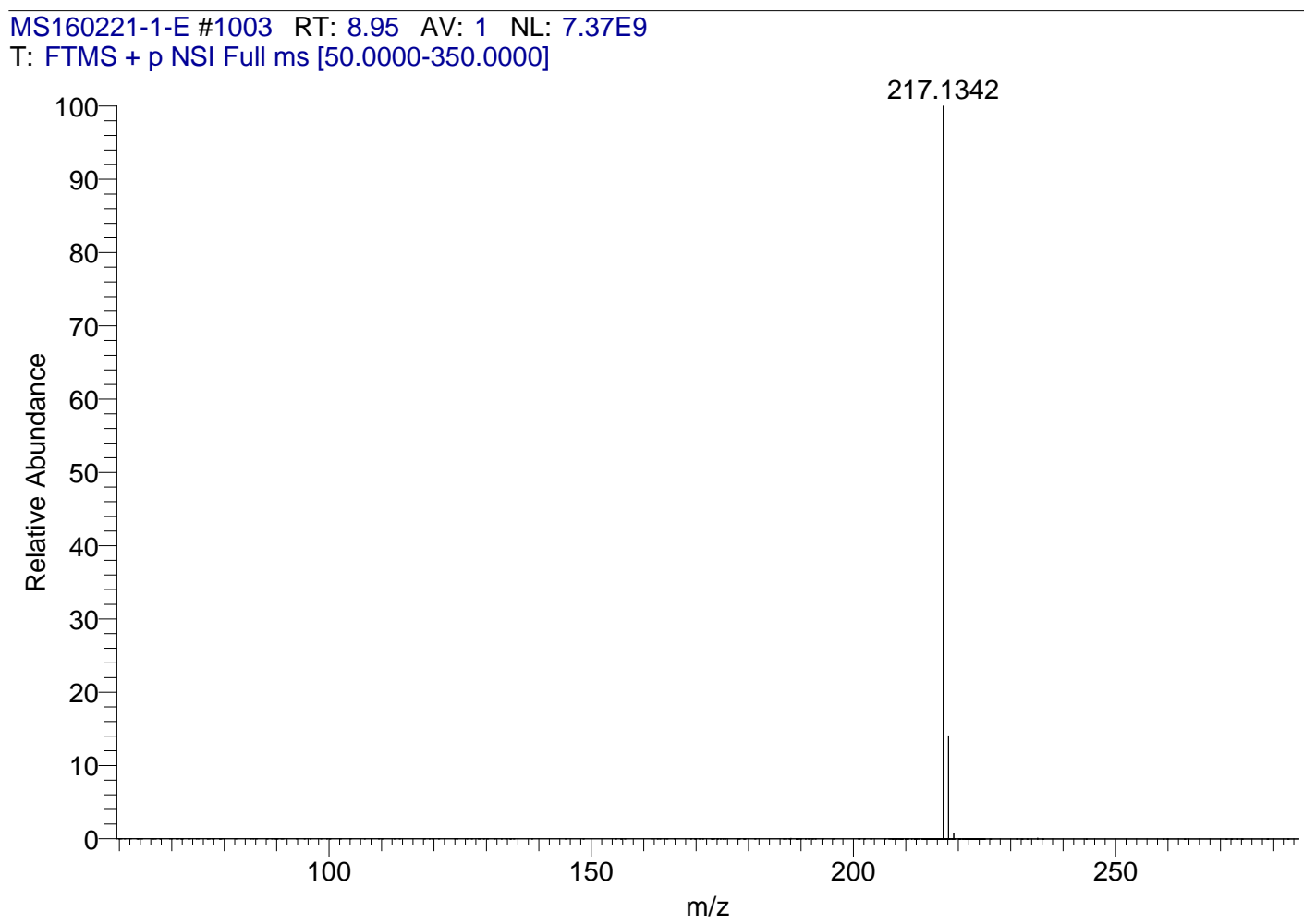

5 e.

MS160221-1-G \#1293-1348 RT: 11.66-12.16 AV: 56 NL: 6.97E9

T: FTMS + p NSI Full ms [50.0000-350.0000]

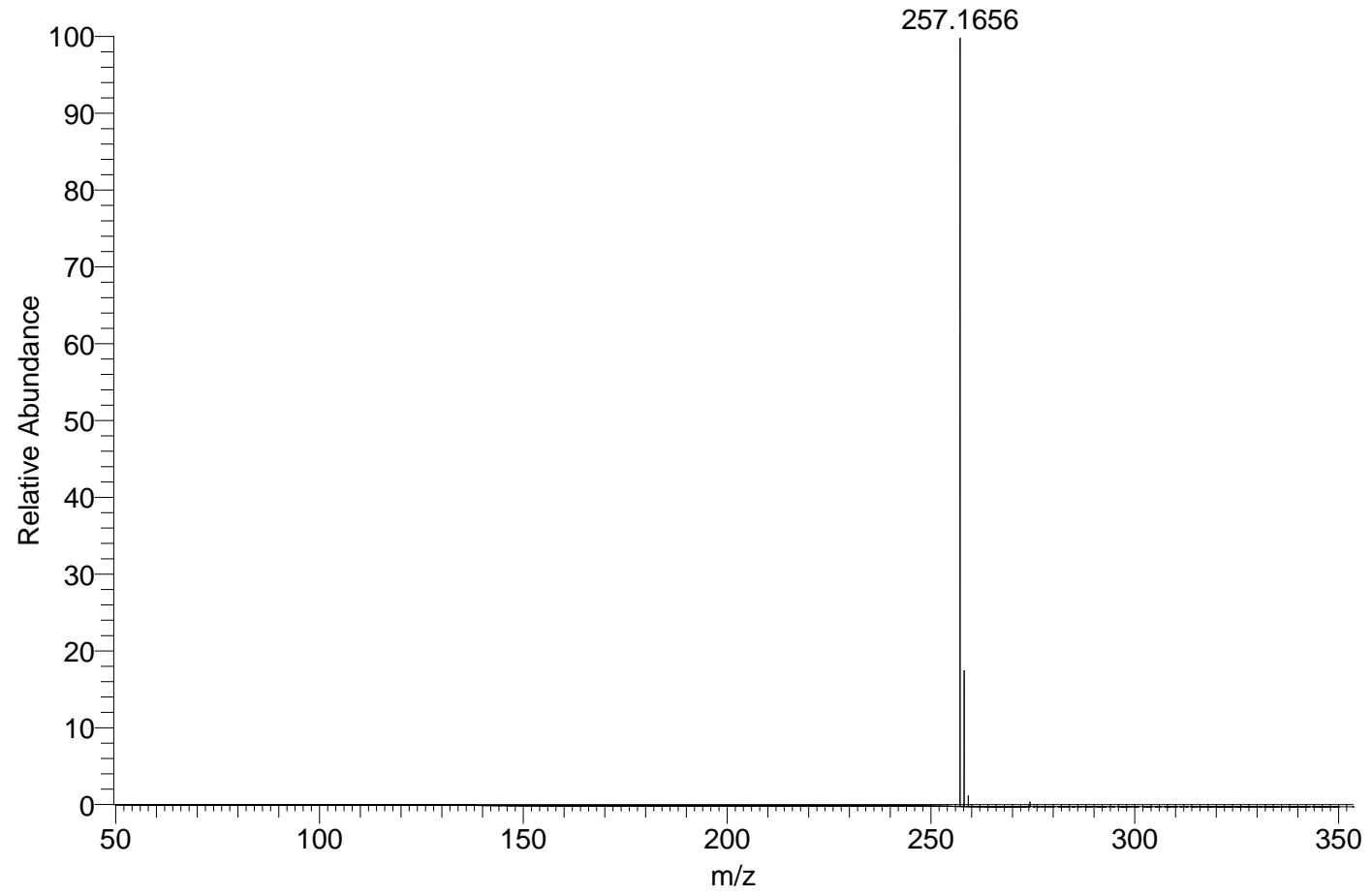


5f. 1-benzyl-5-ethyl-1H-pyrazolo[4,3-b]pyridine

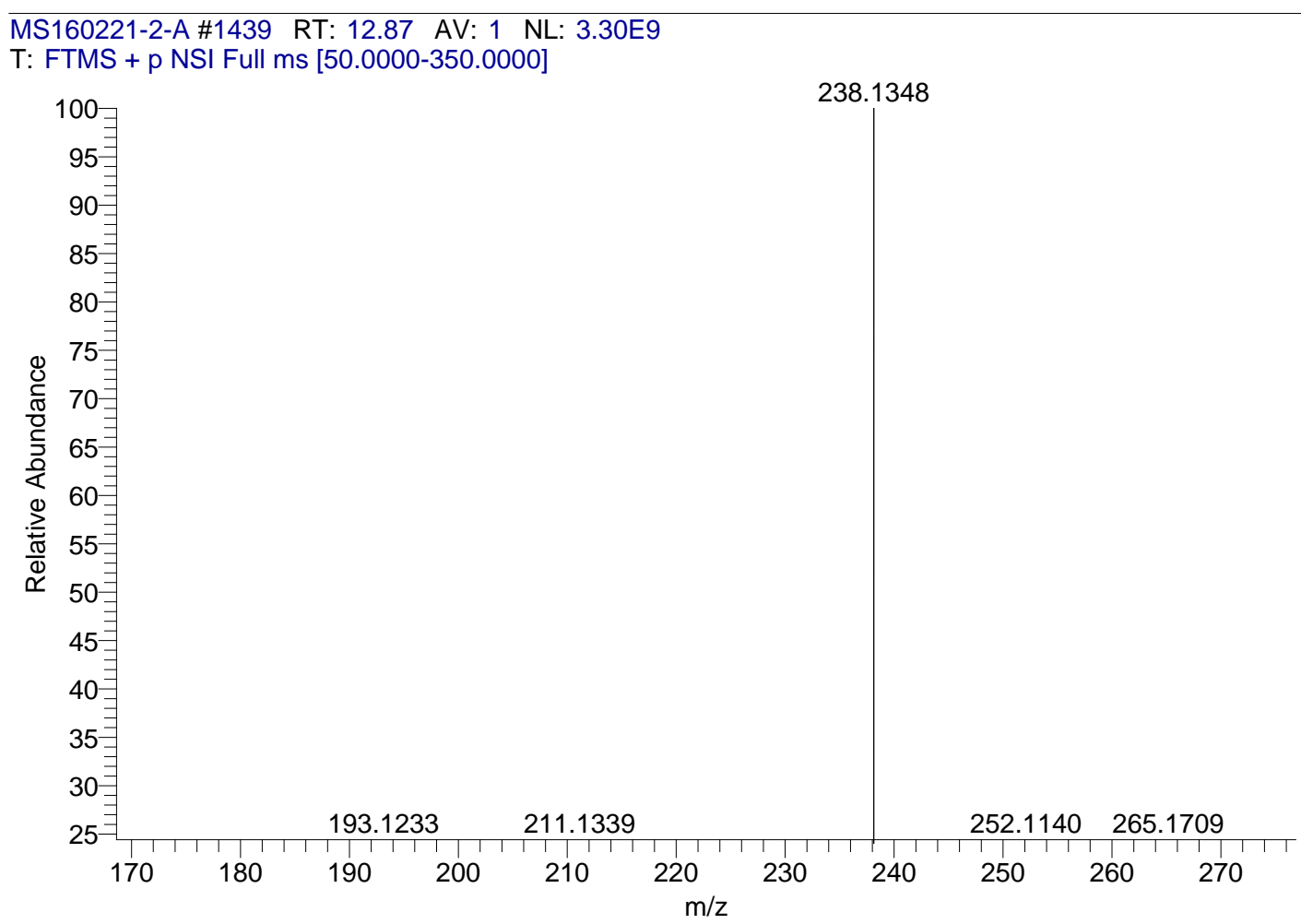

\section{5g. 1-benzyl-5-propyl-1H-pyrazolo[4,3-b]pyridine}

MS160221-2-F \#1063 RT: 9.49 AV: 1 NL: 7.46E9

T: FTMS + p NSI Full ms [50.0000-350.0000]

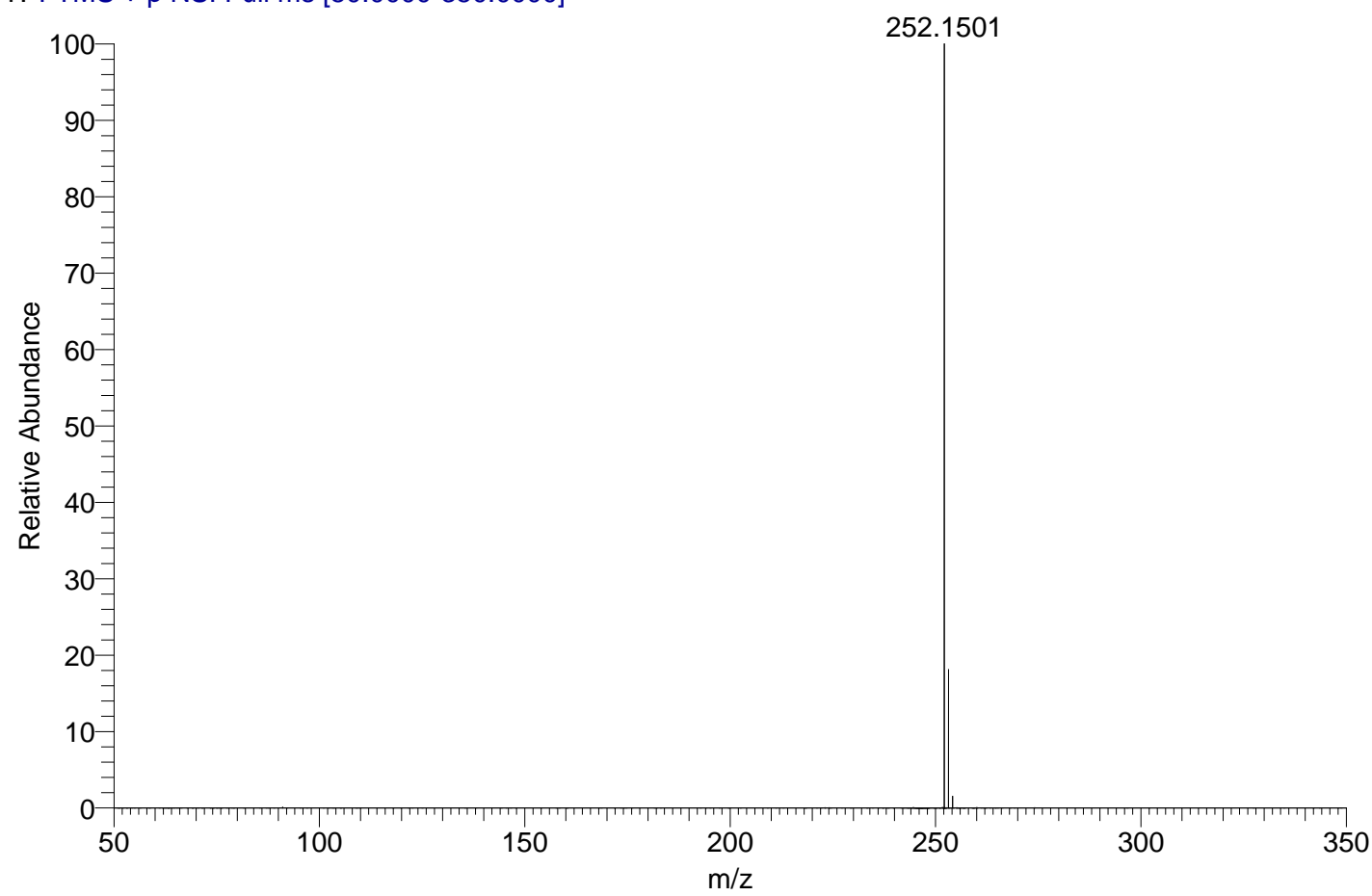


5h. 1-benzyl-5-(pentan-3-yl)-1H-pyrazolo[4,3-b]pyridine

MS160221-1-H \#1164 RT: 10.46 AV: 1 NL: 1.92E9

T: FTMS + p NSI Full ms [50.0000-350.0000]

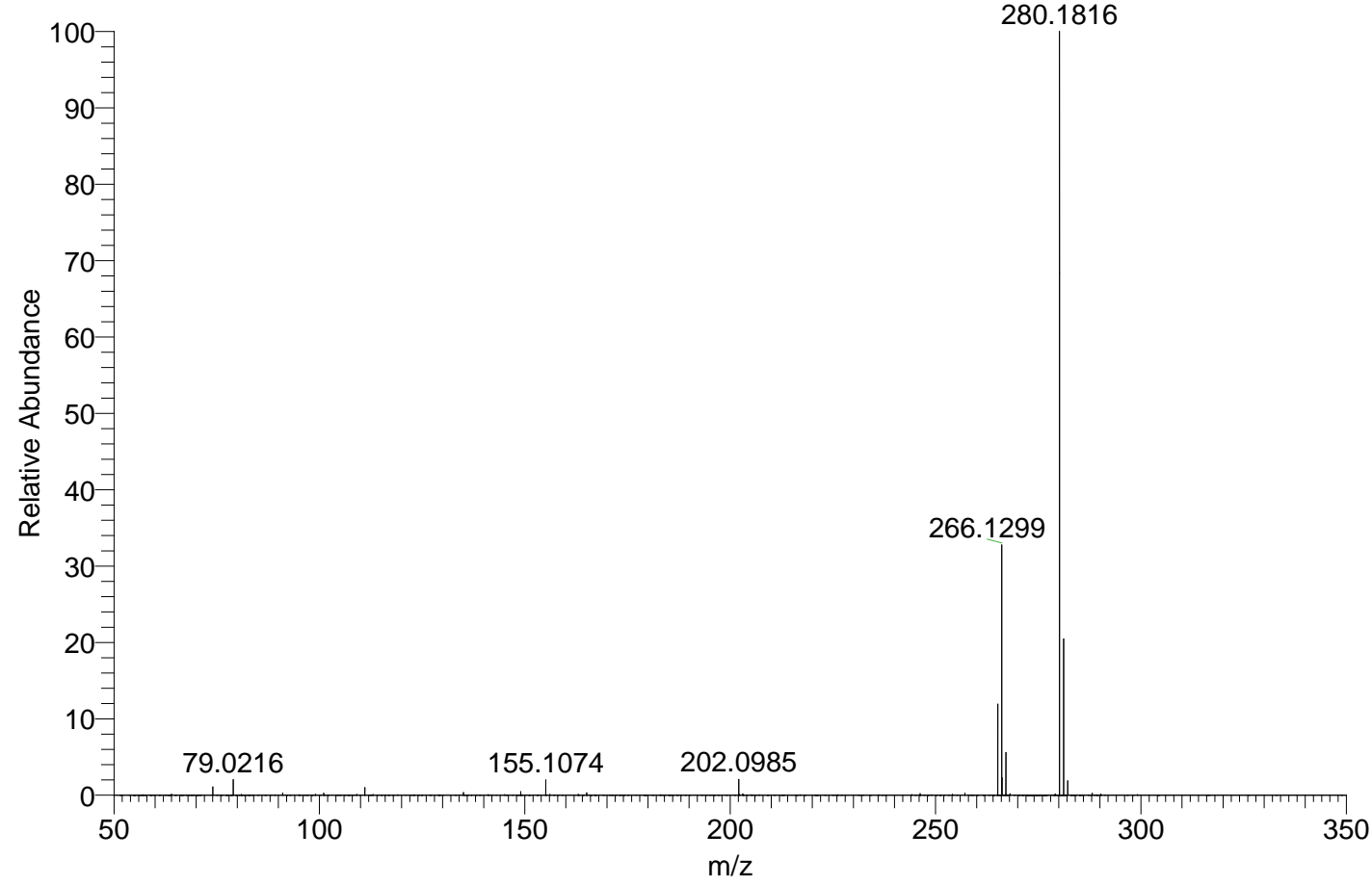

5i. 1-benzyl-5-cyclohexyl-1H-pyrazolo[4,3-b]pyridine

MS160221-2-G \#1196 RT: 11.62 AV: 1 NL: 3.68E9

T: FTMS + p NSI Full ms [50.0000-350.0000]

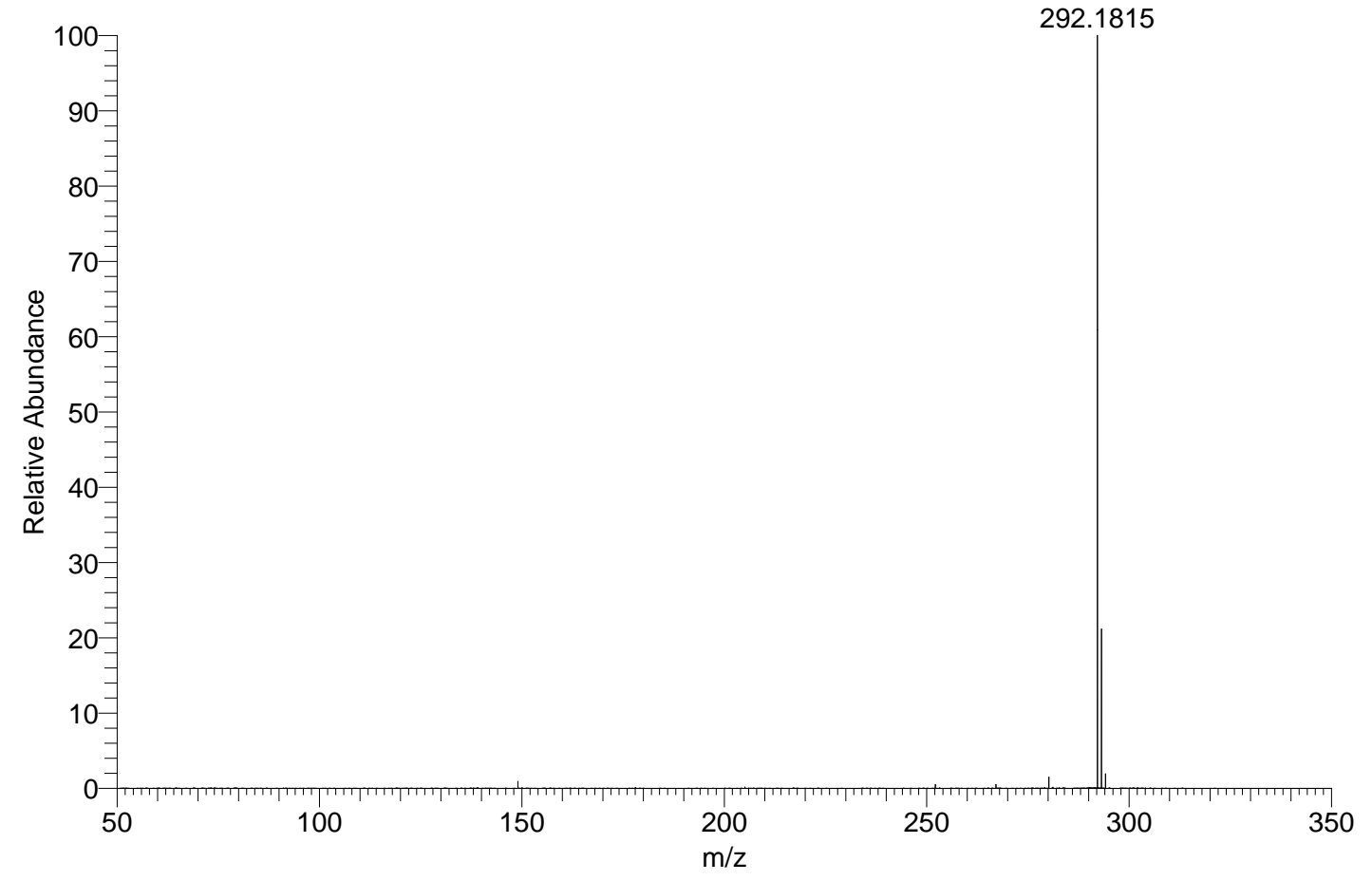


5j. 8-benzyl-1-methyl-1H-pyrrolo[3,2-h]quinoline

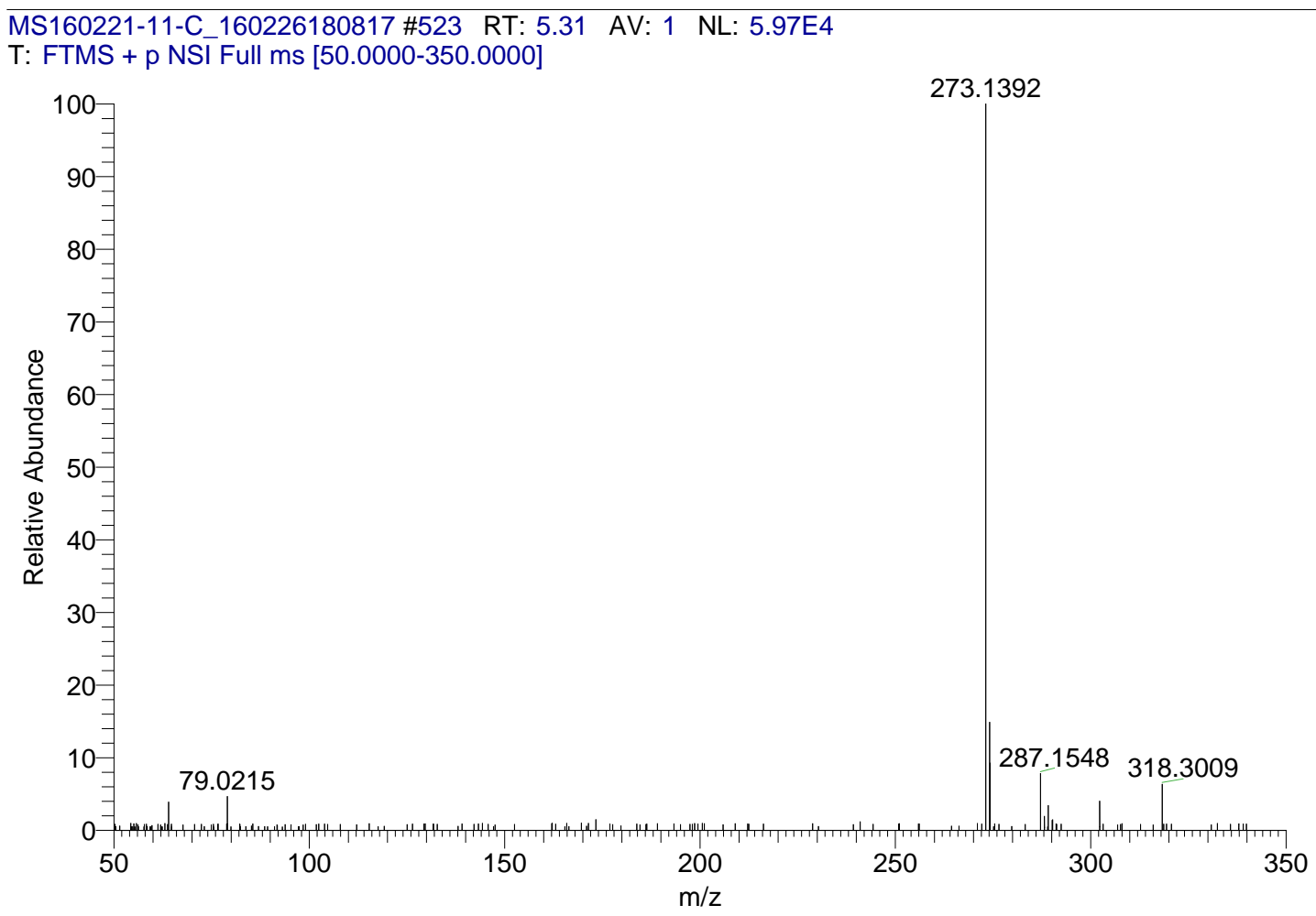

5k. 1,8-dimethyl-1H-pyrrolo[3,2-h]quinoline

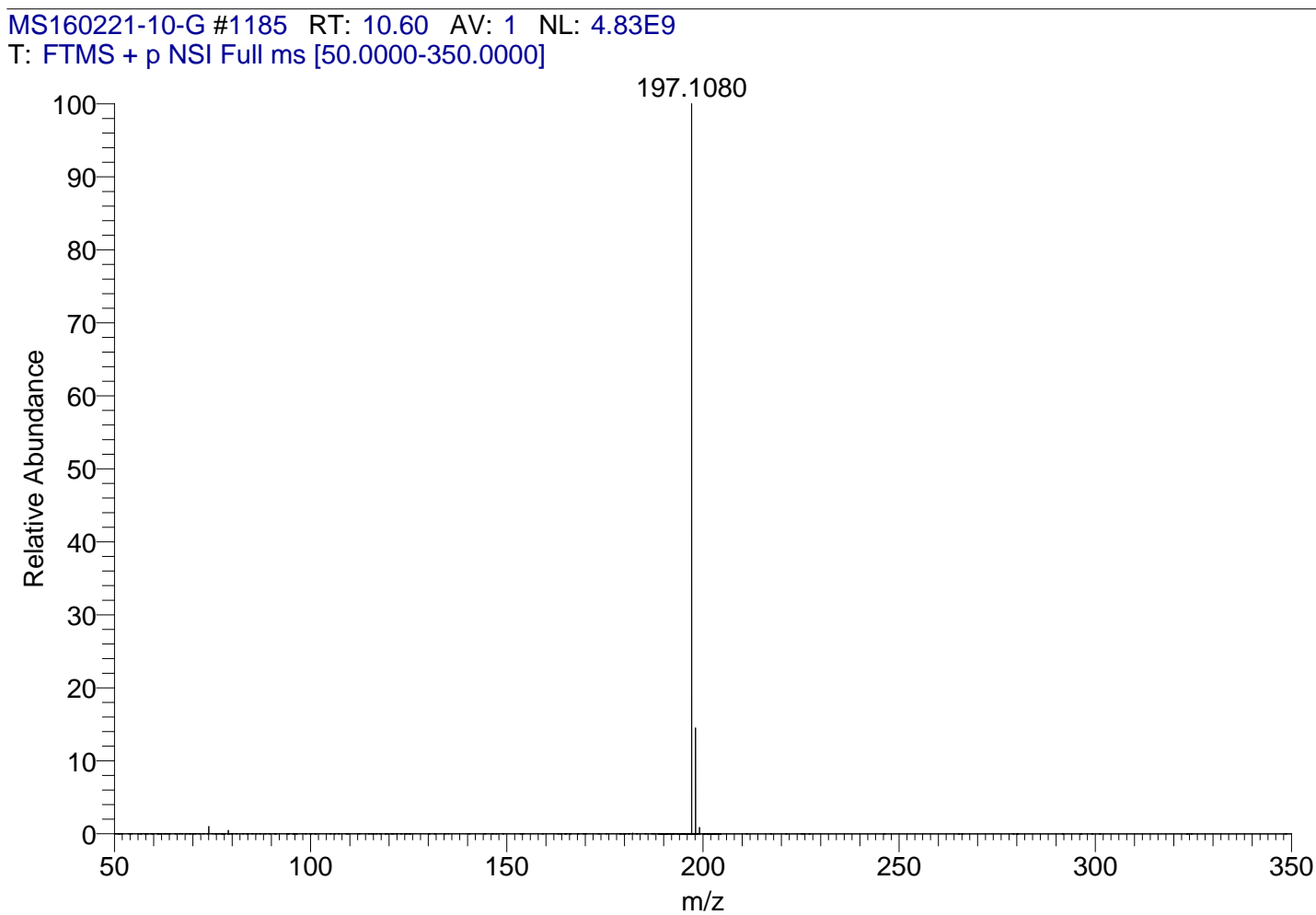


5l. 1-methyl-8-phenethyl-1H-pyrrolo[3,2-h]quinoline

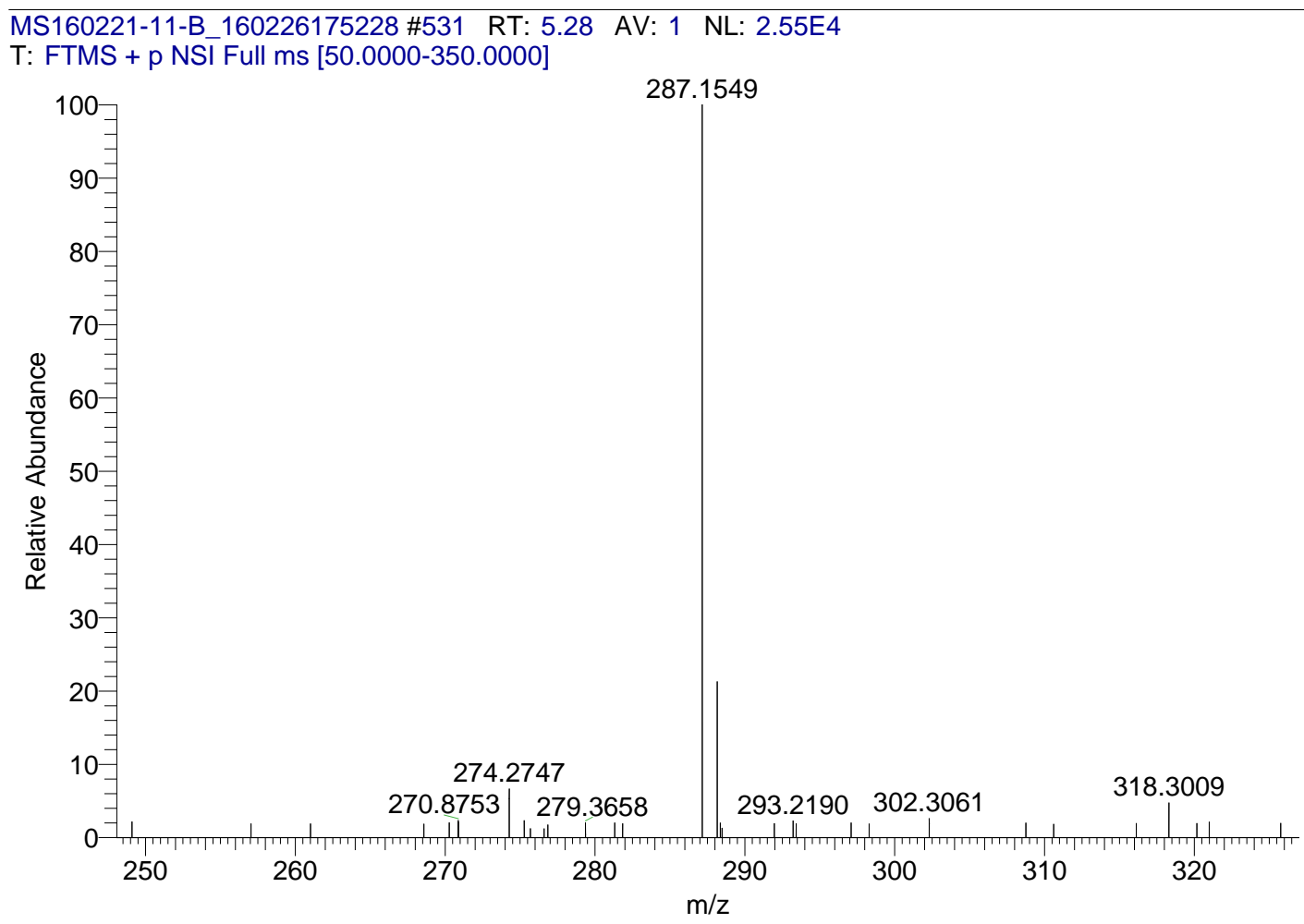

5m. 8-ethyl-1-methyl-1H-pyrrolo[3,2-h]quinolone

MS160221-10-F \#1230 RT: 11.02 AV: 1 NL: 5.20E8

T: FTMS + p NSI Full ms [50.0000-350.0000]

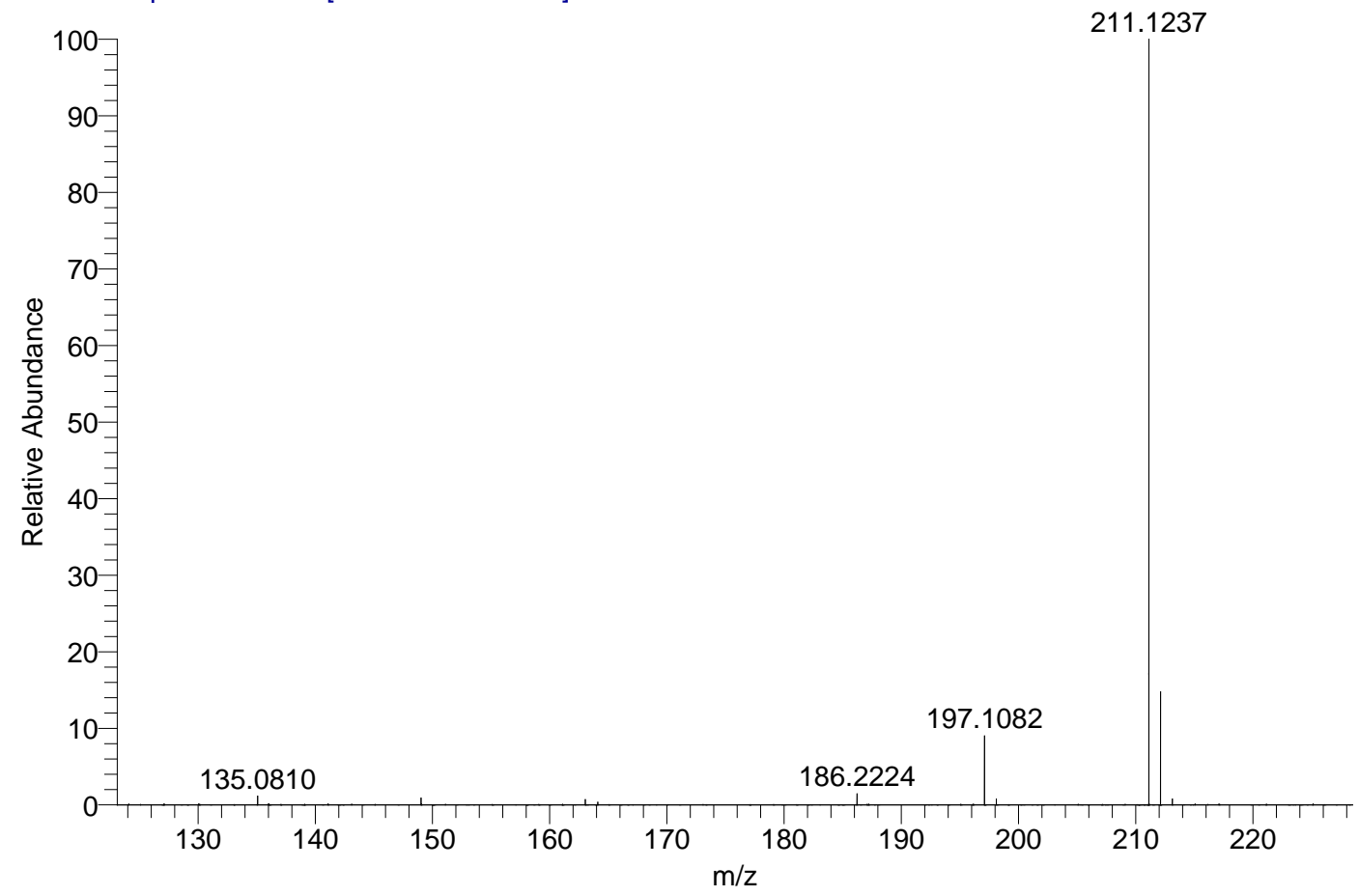


5n. 8-cyclohexyl-1-methyl-1H-pyrrolo[3,2-h]quinolone

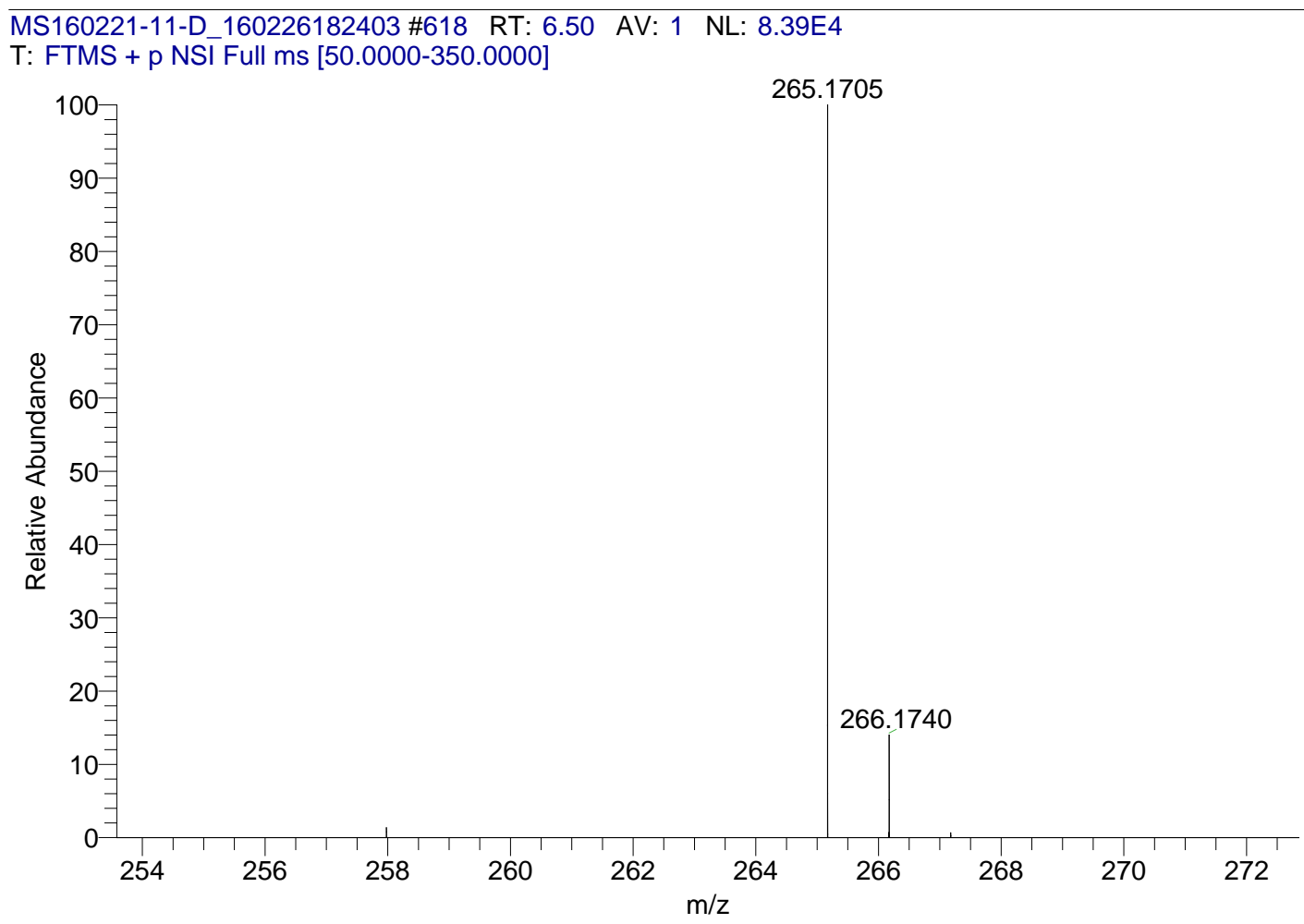

5o. 1-methyl-8-(pentan-3-yl)-1H-pyrrolo[3,2-h]quinolone

MS160221-11-A_160226173644 \#718 RT: 7.07 AV: 1 NL: 1.51E7

T: FTMS + p NSİ Full ms [50.0000-350.0000]

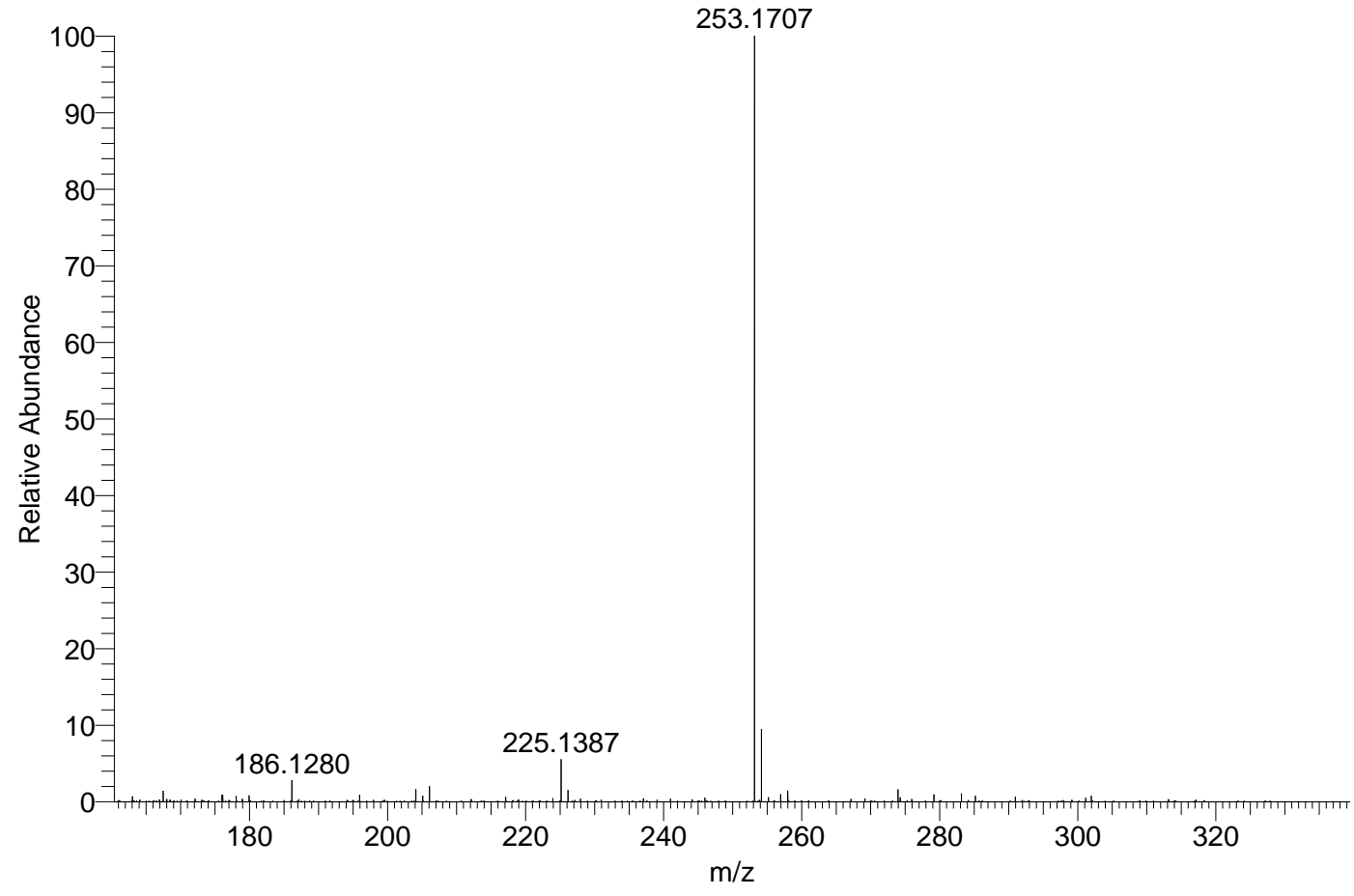


5p. 1-methyl-8-(pentan-3-yl)-1H-pyrrolo[3,2-h]quinolone

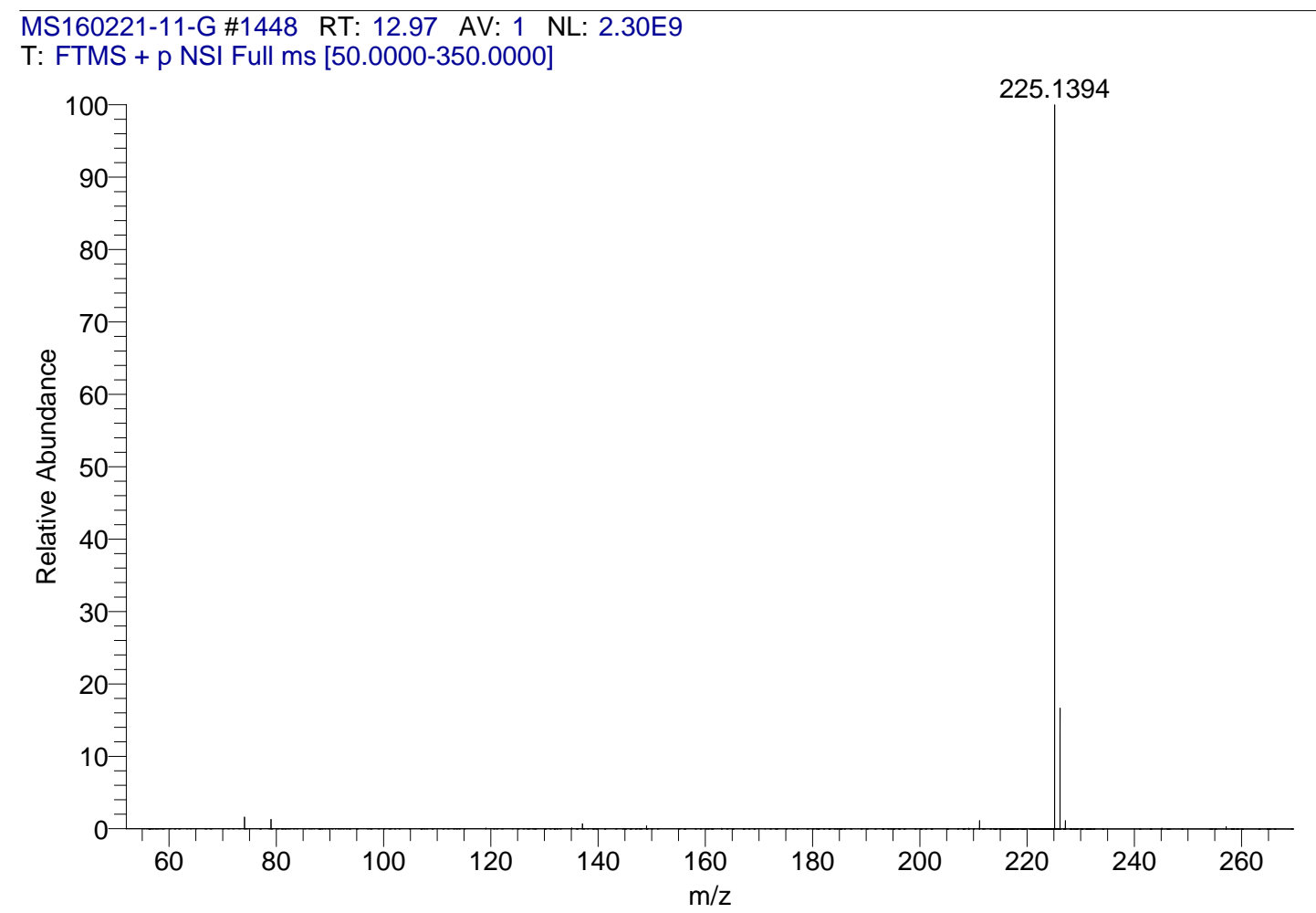

5q. 1-methyl-7-propyl-1H-pyrrolo[2,3-f]quinoline

MS160221-10-D \#1013 RT: 9.07 AV: 1 NL: 7.41E9

T: FTMS + p NSI Full ms [50.0000-350.0000]

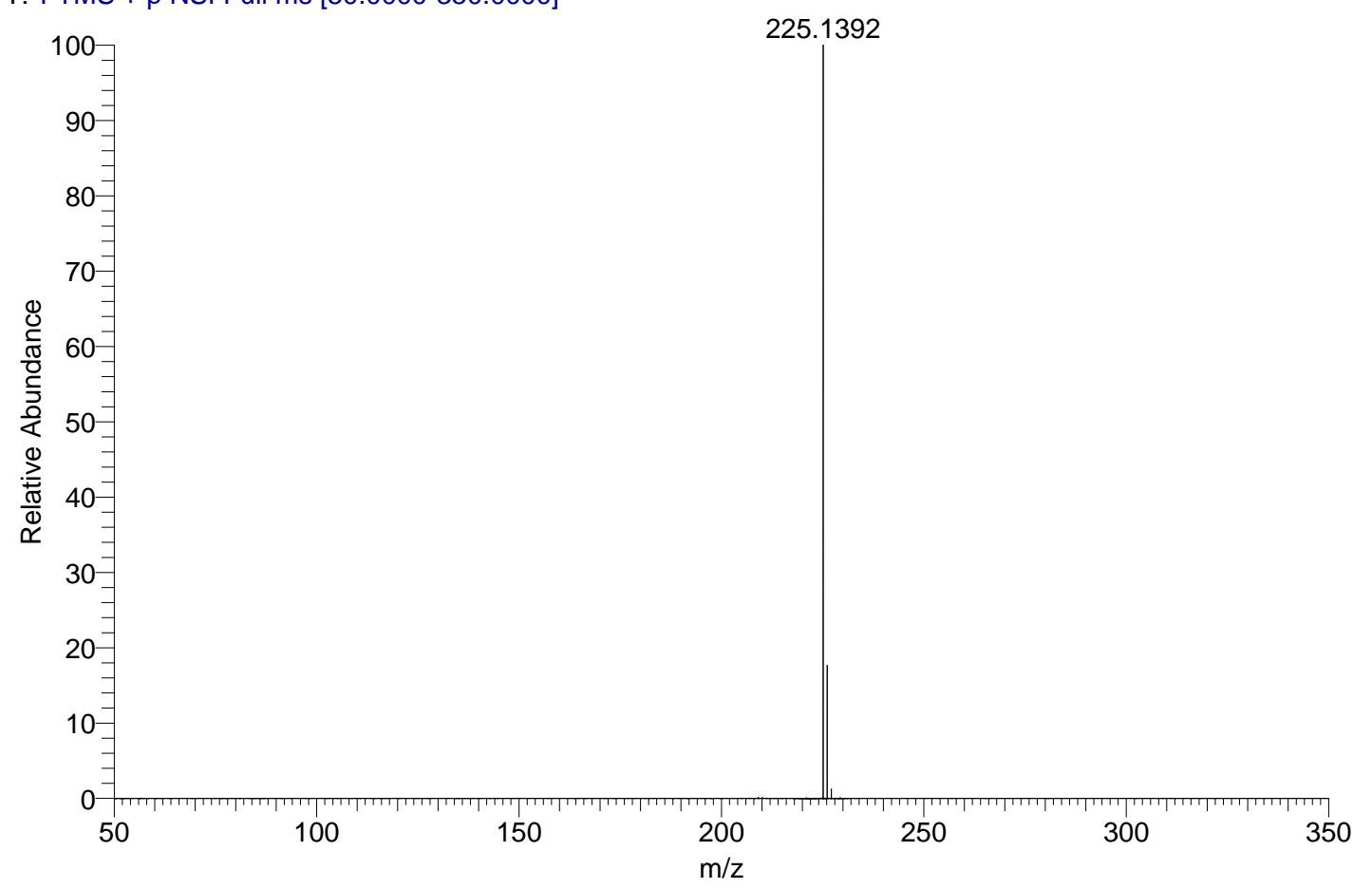


5r. 1-methyl-7-(pentan-3-yl)-1H-pyrrolo[2,3-f]quinoline

MS160221-10-B \#1099-1142 RT: 9.91-10.30 AV: 44 NL: 5.60E9

T: FTMS + p NSI Full ms [50.0000-350.0000]

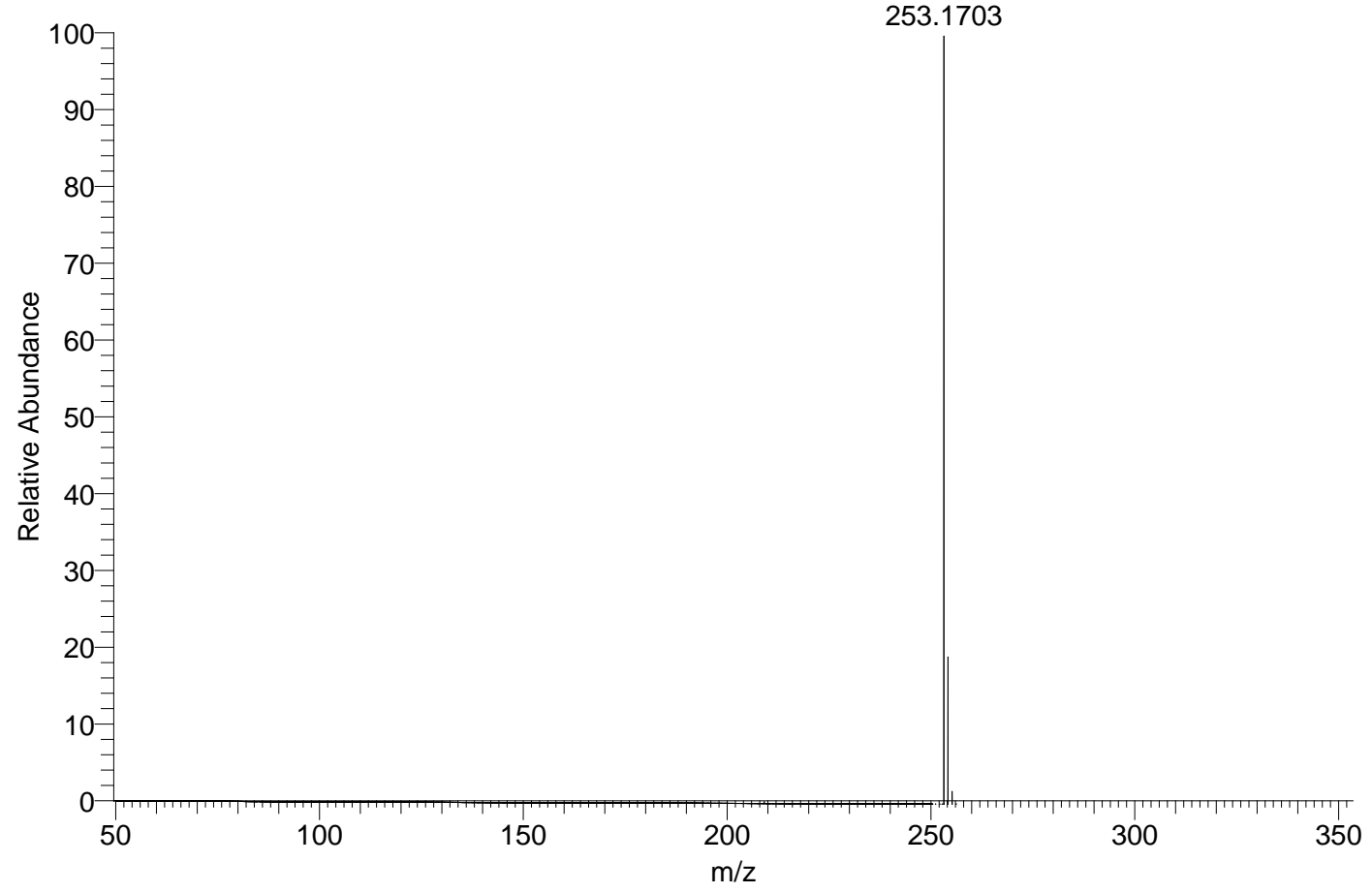

5s. 7-cyclohexyl-1-methyl-1H-pyrrolo[2,3-f]quinoline

MS160221-10-C \#1254 RT: 11.23 AV: 1 NL: 6.36E9

T: FTMS + p NSI Full ms [50.0000-350.0000]

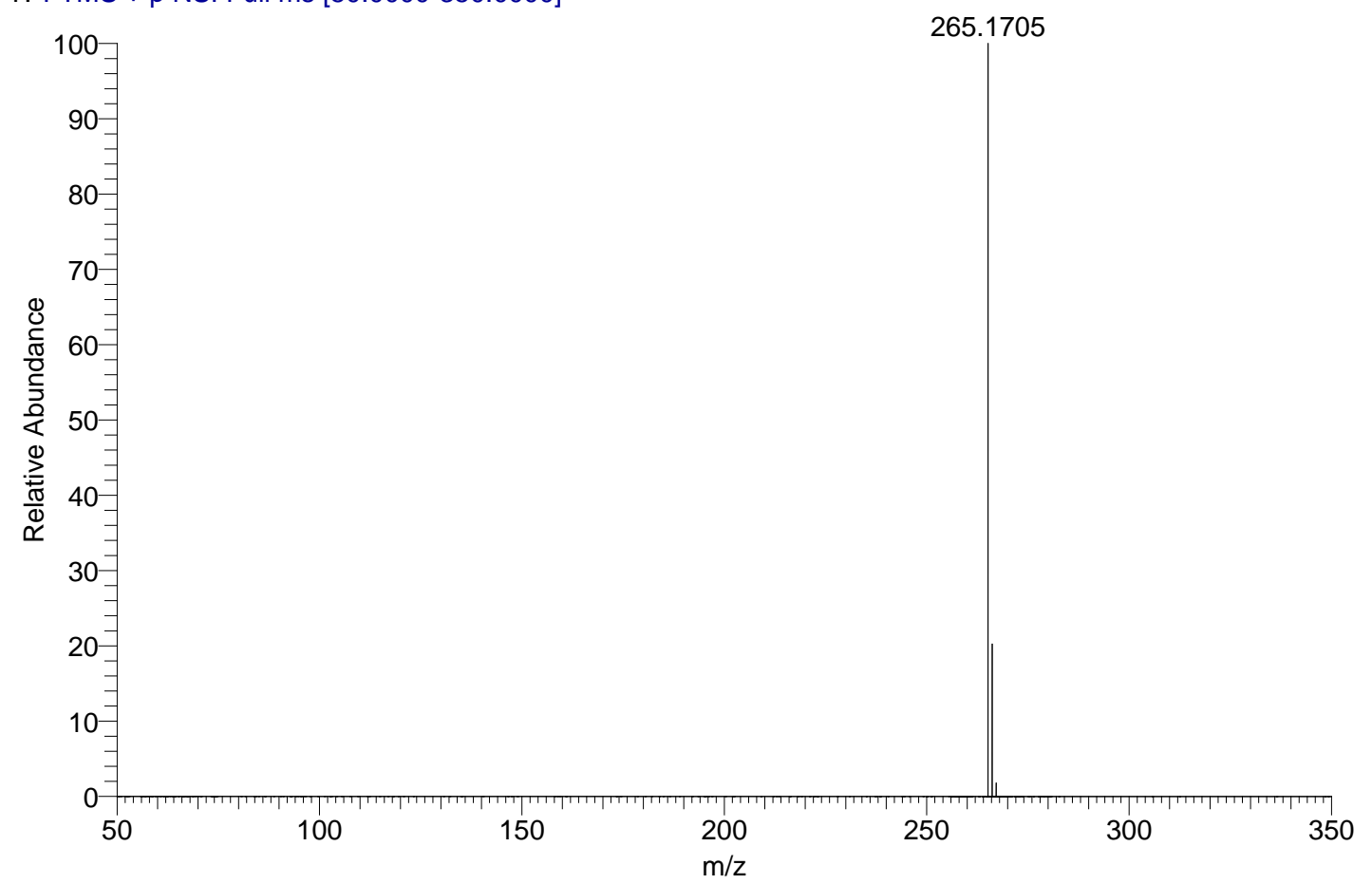


5t. 7-cyclohexyl-3-methyl-3H-pyrazolo[4,3-f]quinolone

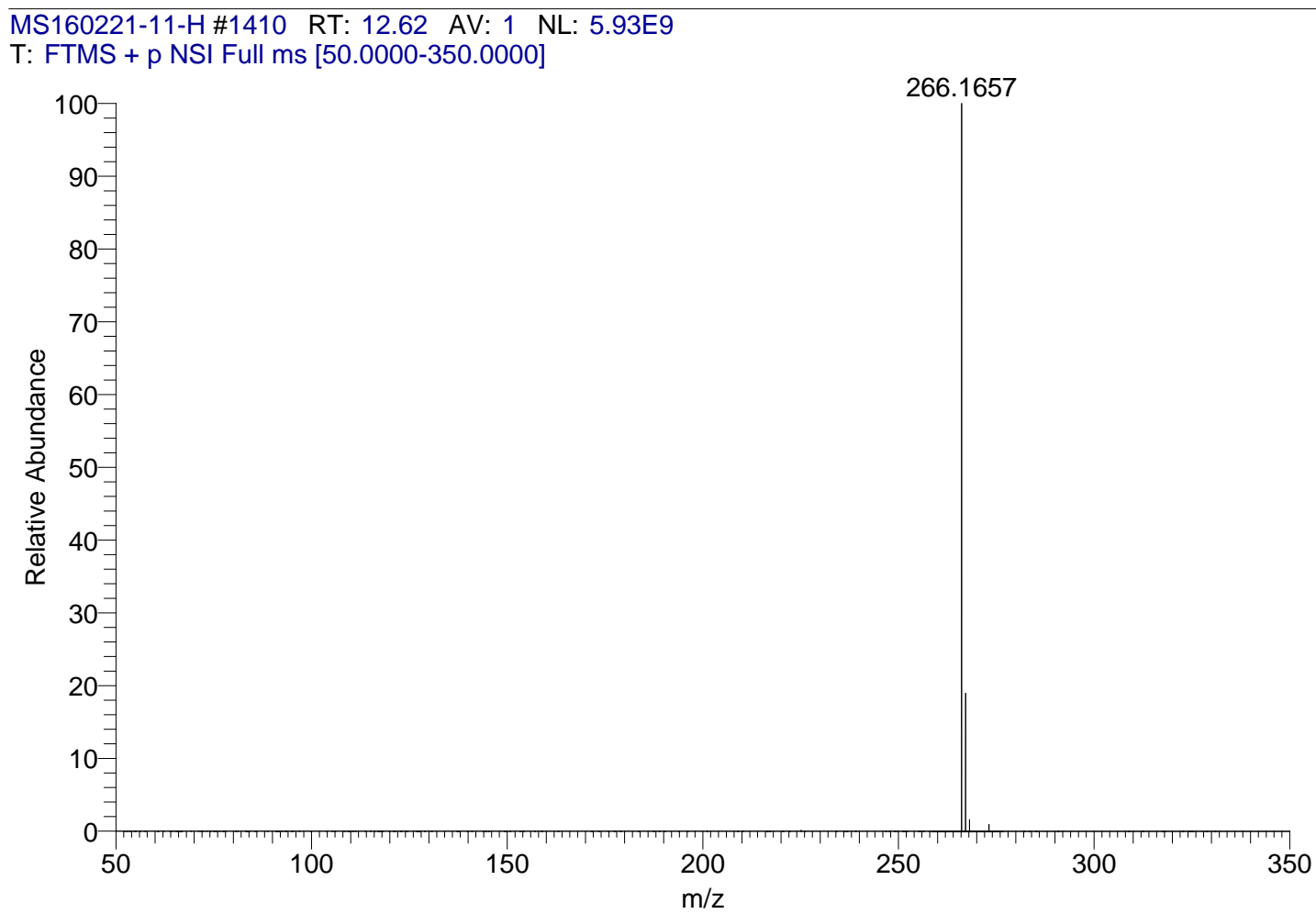

5u. 7-ethyl-3-methyl-3H-pyrazolo[4,3-f]quinolone

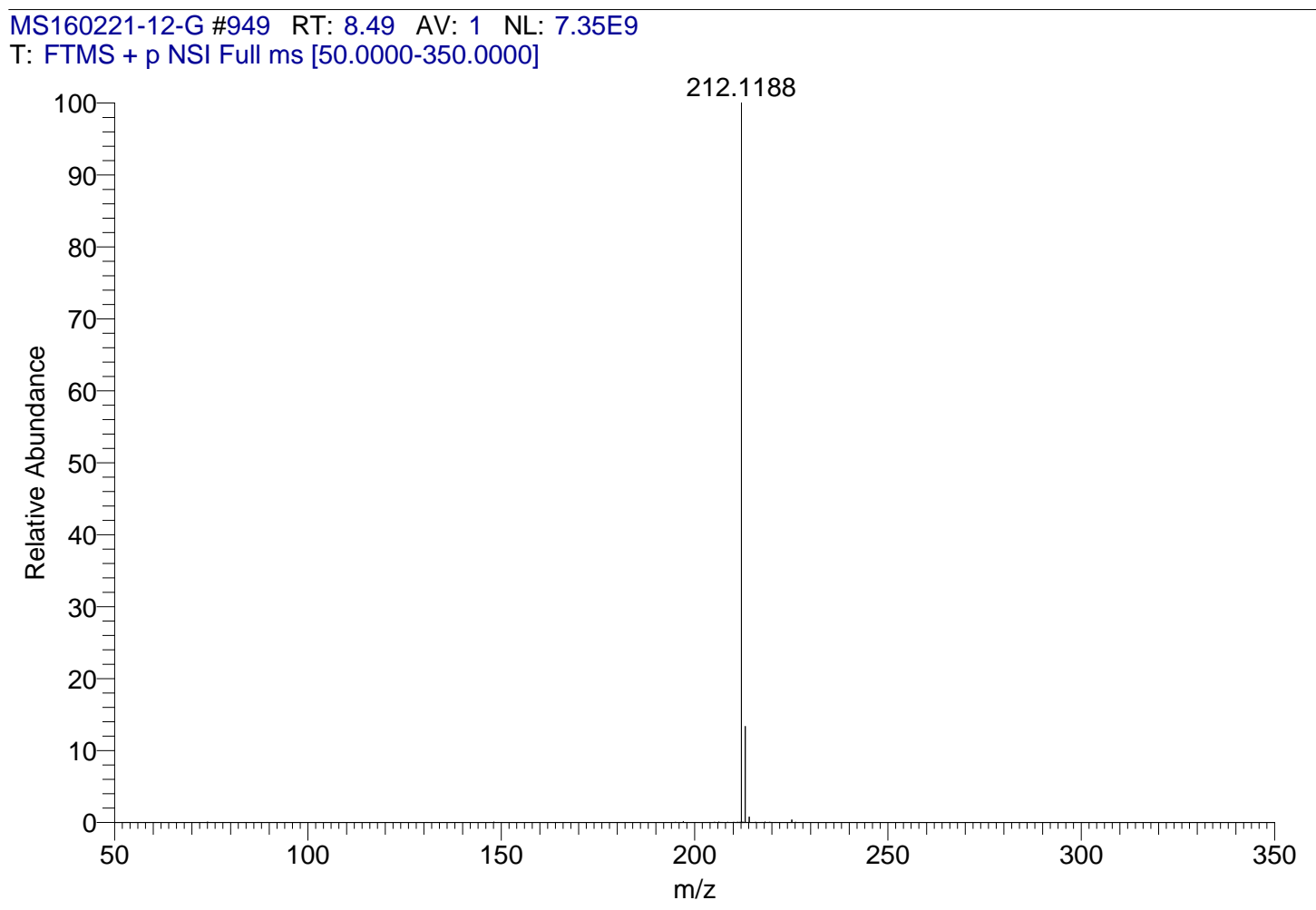


5v. 3-methyl-7-propyl-3H-pyrazolo[4,3-f]quinolone

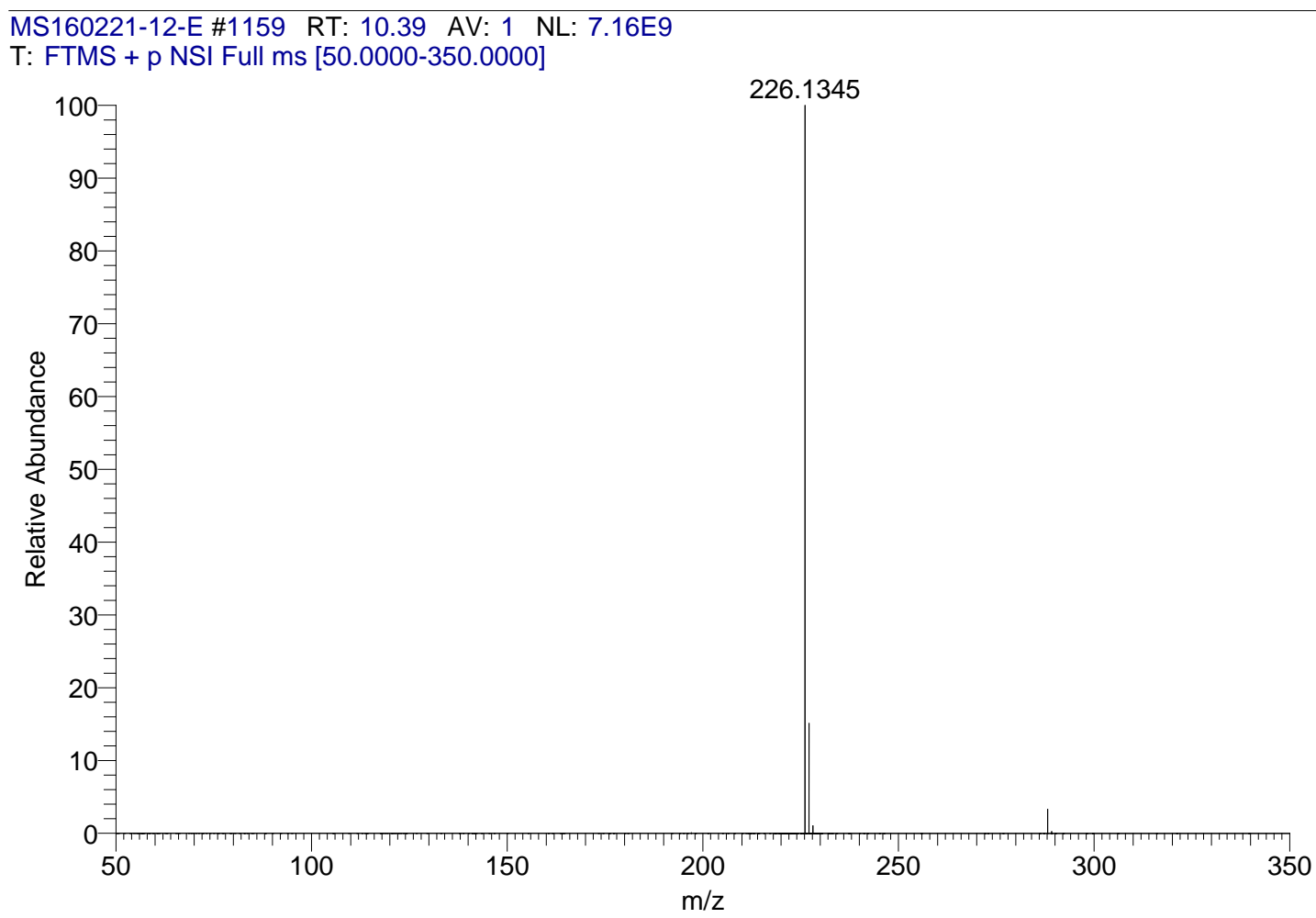

5w. 3-methyl-7-(pentan-3-yl)-3H-pyrazolo[4,3-f]quinolone

MS160221-12-F \#1305 RT: 11.66 AV: 1 NL: 7.70E9

T: FTMS + p NSI Full ms [50.0000-350.0000]

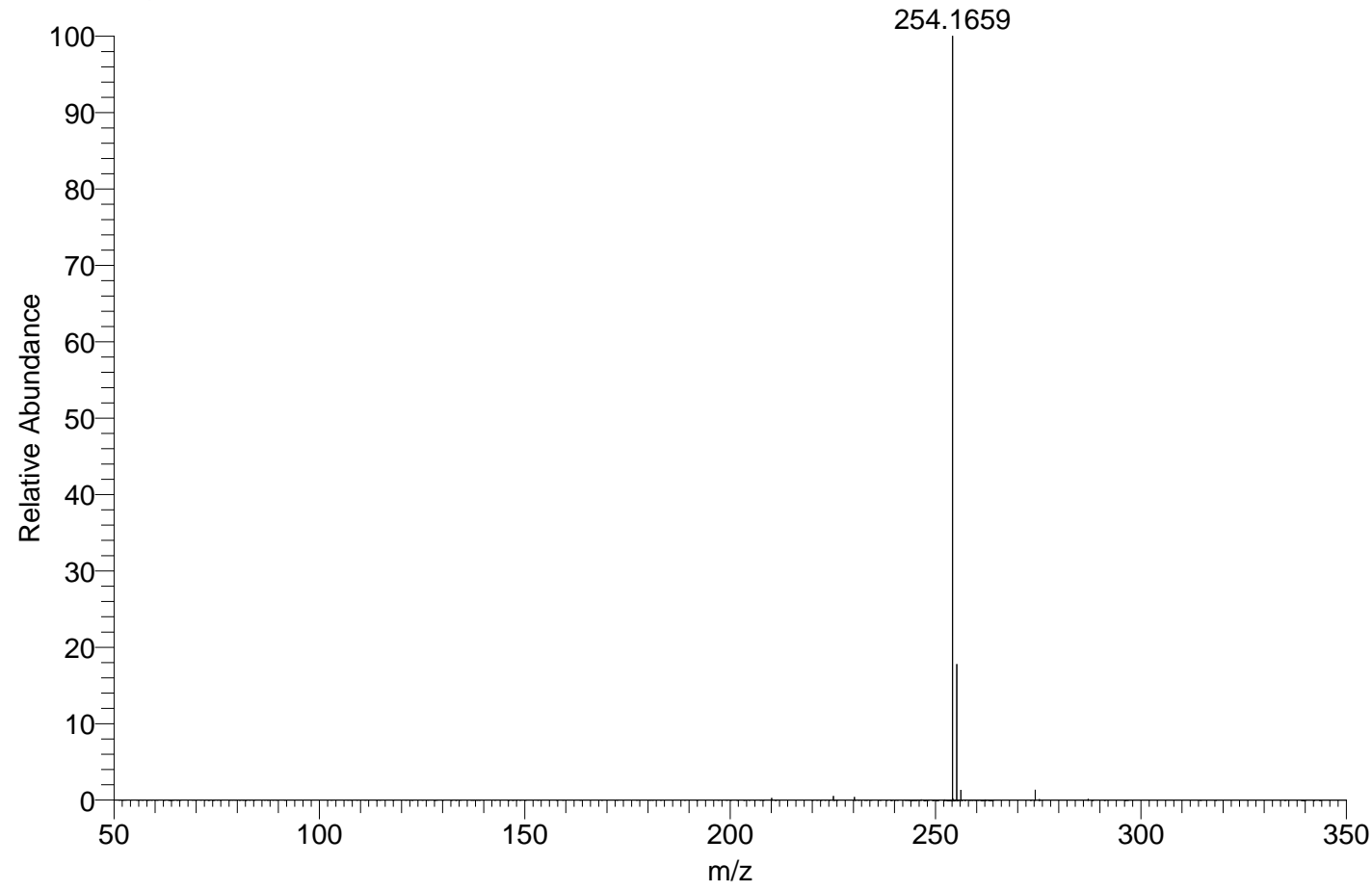


$5 x$.

MS160221-2-H \#1026-1042 RT: 9.26-9.41 AV: 17 NL: 4.98E9

T: FTMS + p NSI Full ms [50.0000-350.0000]

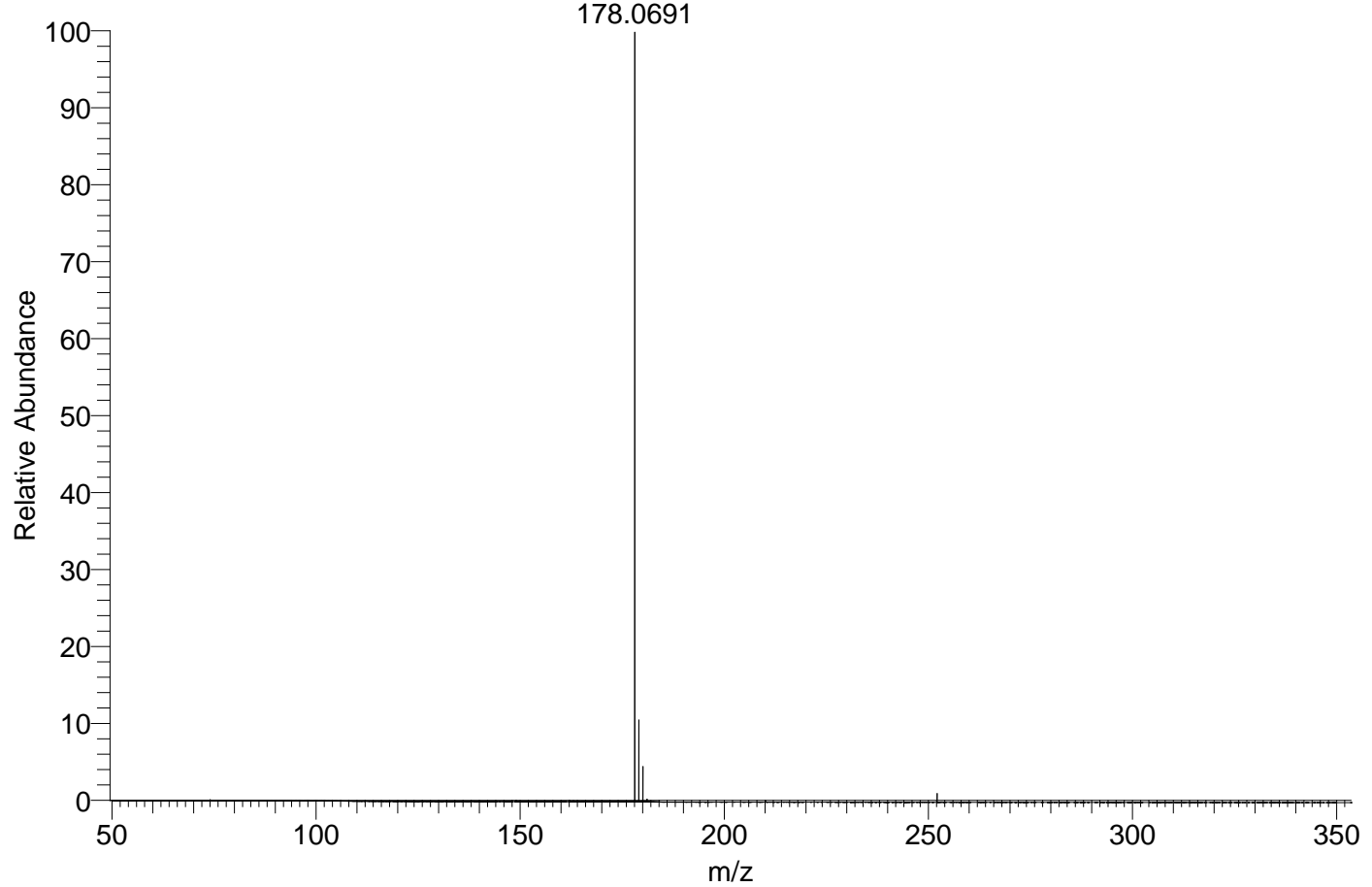




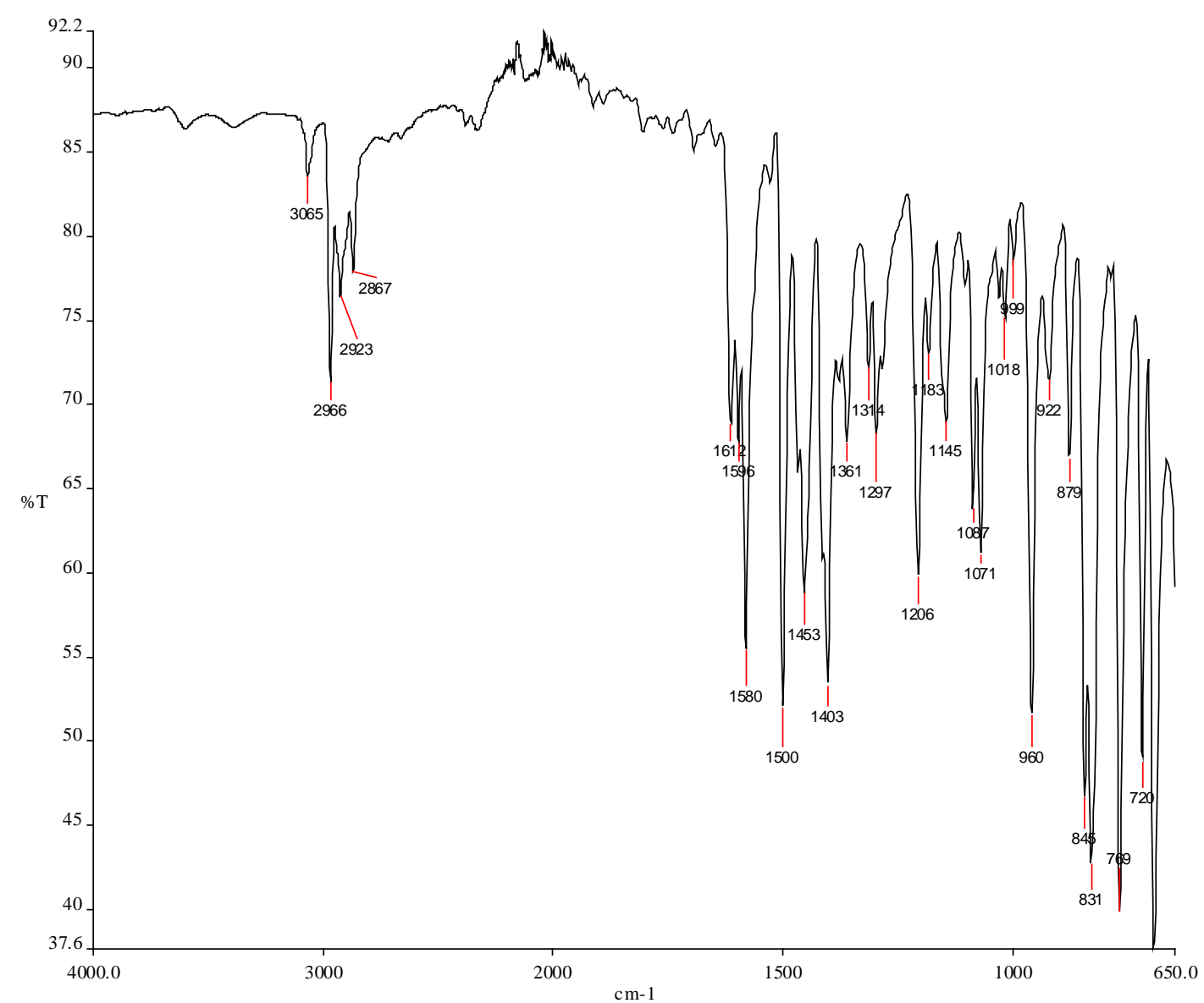

1

Compound 40-1. 7-isopropyl-1-phenyl-1H-pyrazolo[3,4-f]quinolone 


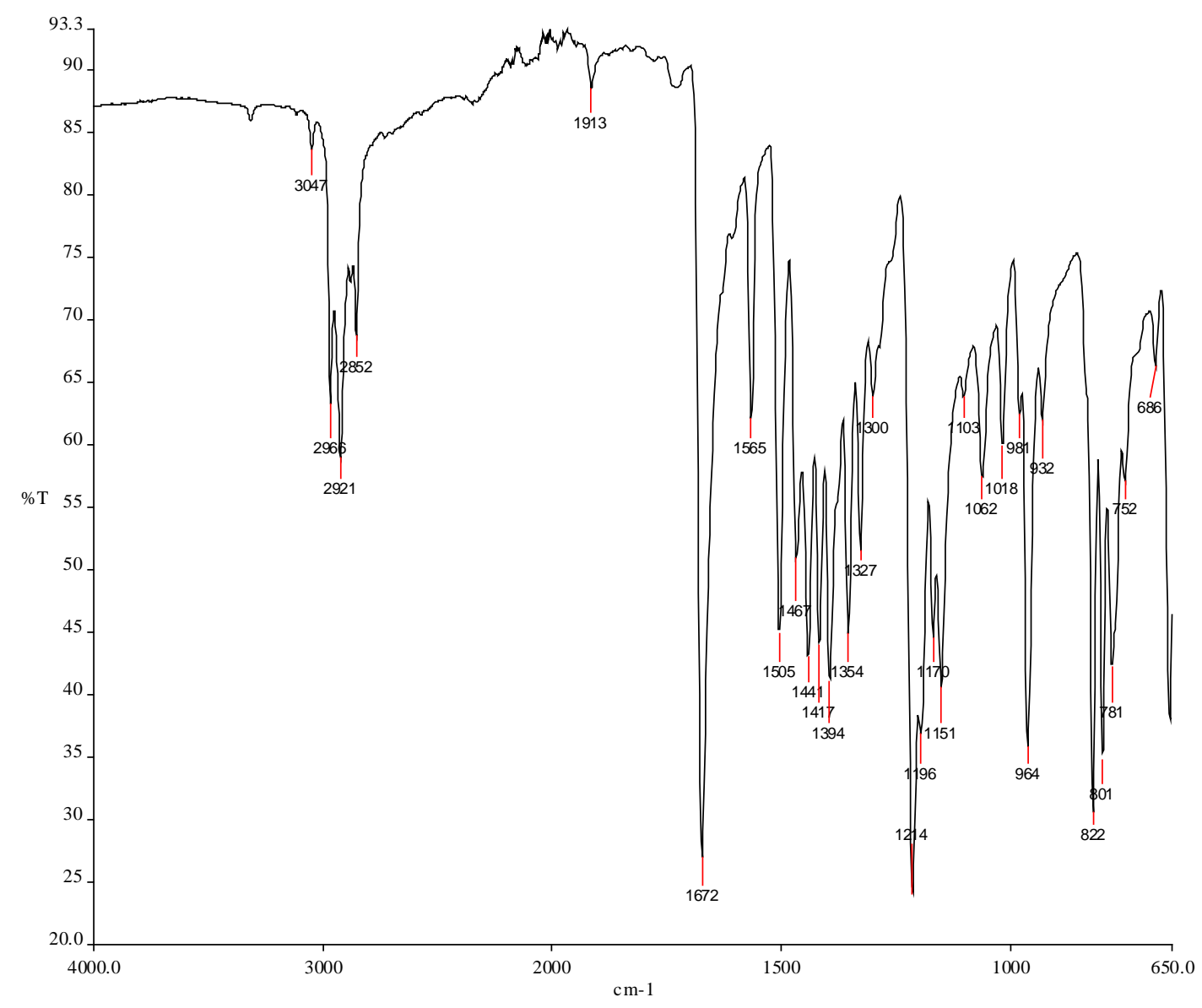

2

Compound 5c. 1-(5-ethyl-1-methyl-1H-pyrrolo[3,2-b]pyridin-2-yl)ethanone 


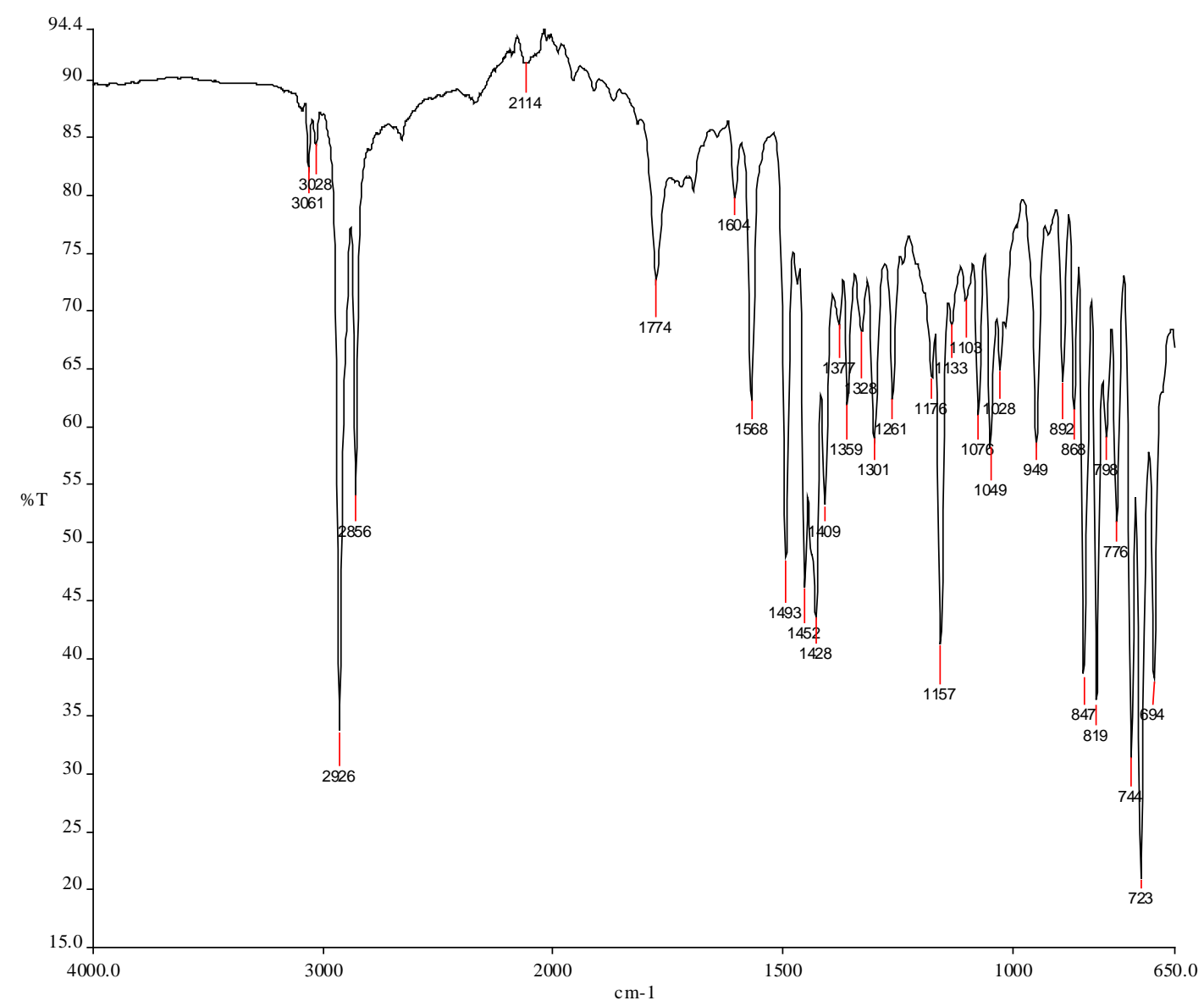

3

Compound 5i. 1-benzyl-5-cyclohexyl-1H-pyrazolo[4,3-b]pyridine 


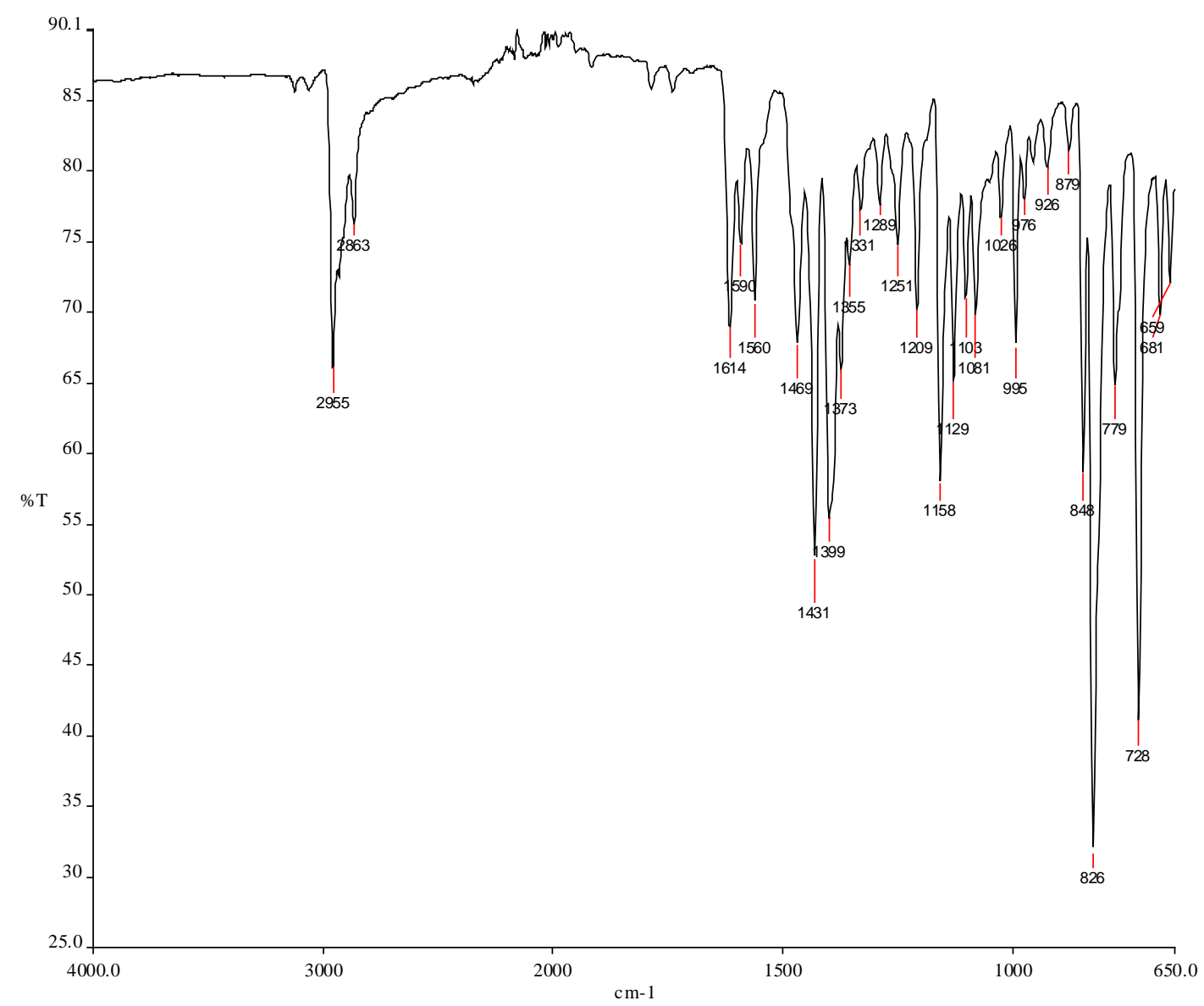

4

Compound 4m. 7-isopropyl-2-methyl-2H-pyrazolo[3,4-f]quinolone 


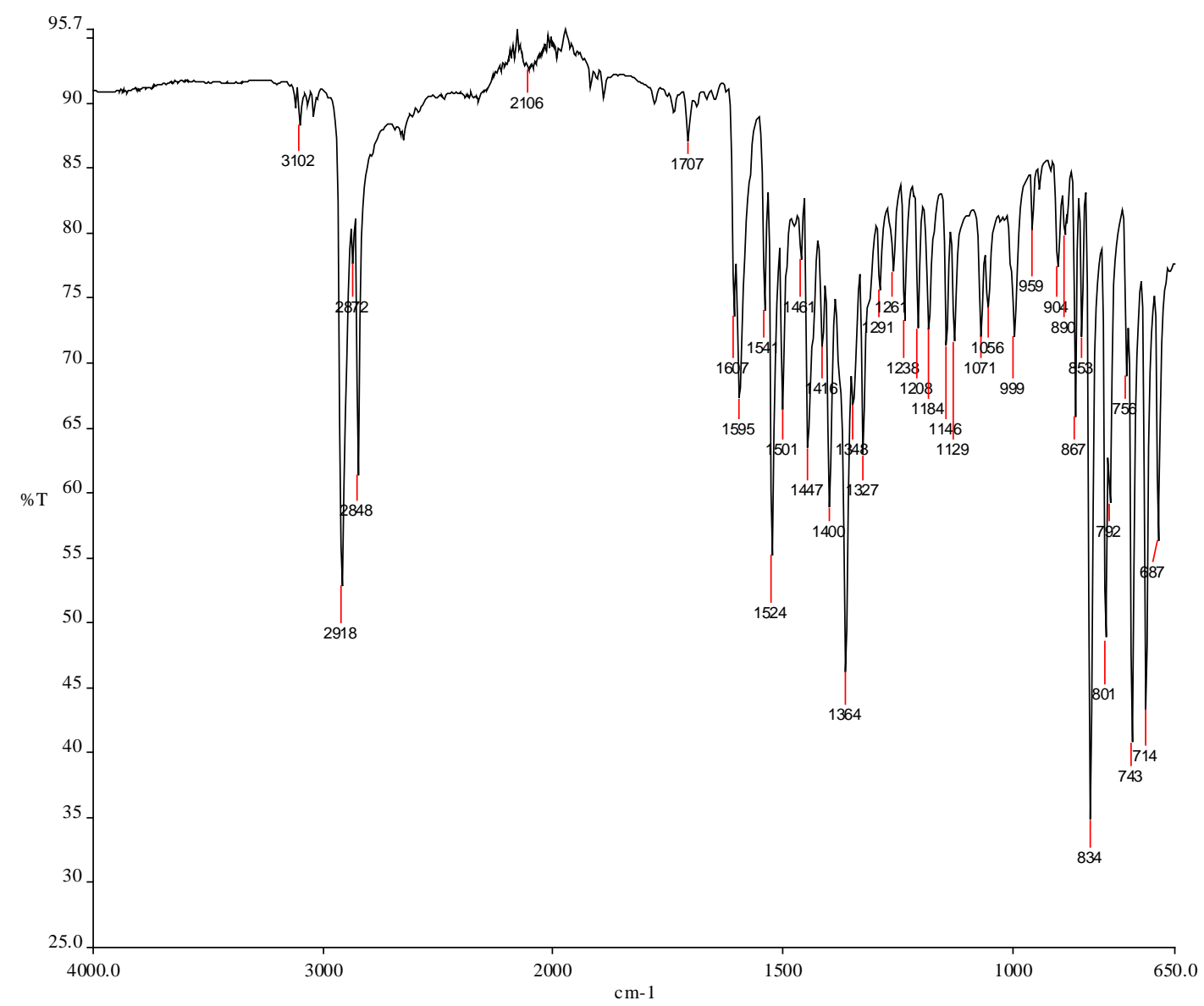

5

Compound 5n. 8-cyclohexyl-1-methyl-1H-pyrrolo[3,2-h]quinolone 